\title{
Manuela Tillmanns
}

\section{Intergeschlechtlichkeit}

Impulse für die Beratung

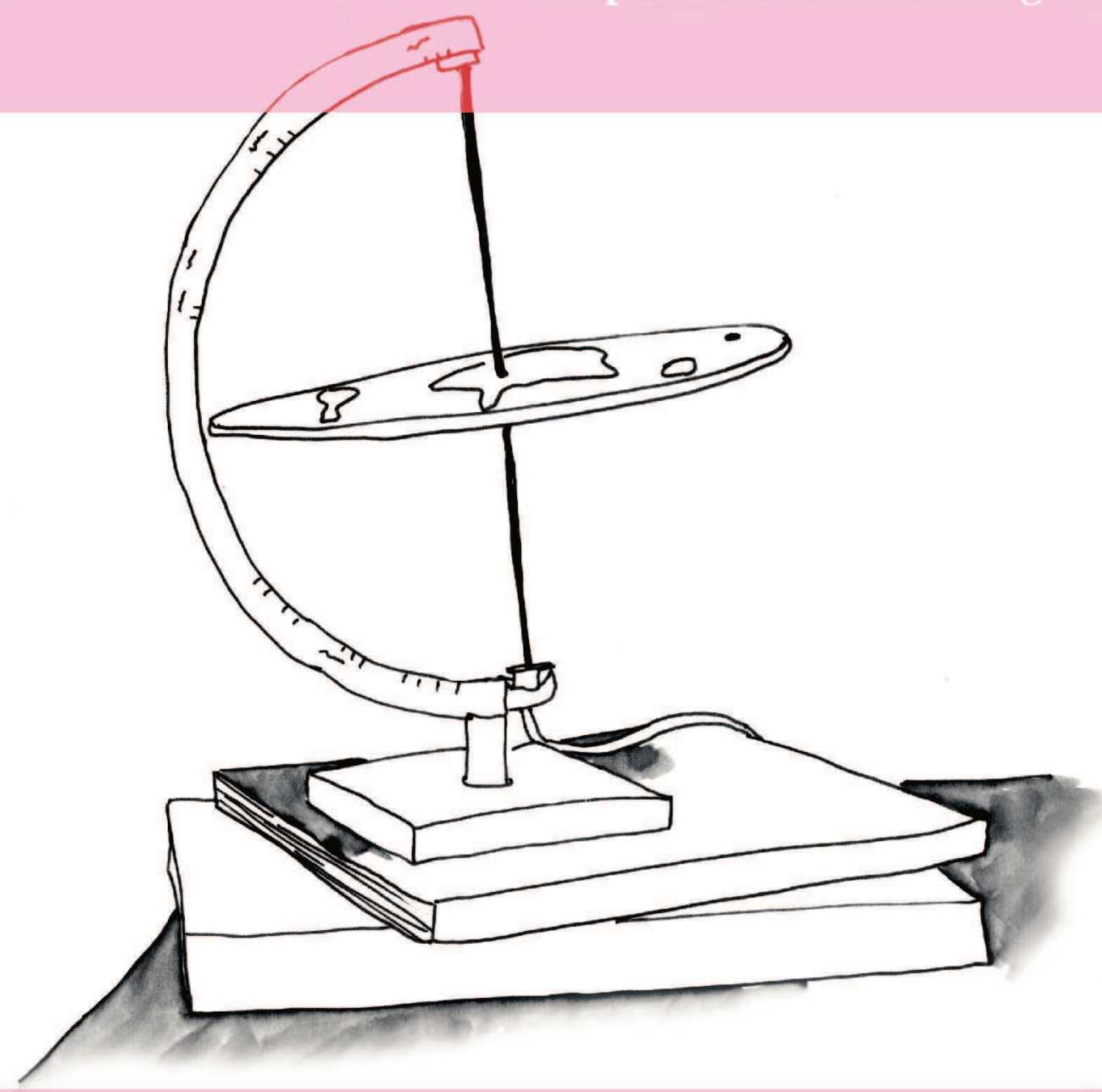




\section{Manuela Tillmanns \\ Intergeschlechtlichkeit}


$\mathrm{D}$ ie Reihe »Angewandte Sexualwissenschaft « sucht den Dialog: Sie ist interdisziplinär angelegt und zielt insbesondere auf die Verbindung von Theorie und Praxis. Vertreter_innen aus wissenschaftlichen Institutionen und aus Praxisprojekten wie Beratungsstellen und Selbstorganisationen kommen auf Augenhöhe miteinander ins Gespräch. Auf diese Weise sollen die bisher oft langwierigen Transferprozesse verringert werden, durch die praktische Erfahrungen erst spät in wissenschaftlichen Institutionen Eingang finden. Gleichzeitig kann die Wissenschaft so zur Fundierung und Kontextualisierung neuer Konzepte beitragen.

Der Reihe liegt ein positives Verständnis von Sexualität zugrunde. Der Fokus liegt auf der Frage, wie ein selbstbestimmter und wertschätzender Umgang mit Geschlecht und Sexualität in der Gesellschaft gefördert werden kann. Sexualität wird dabei in ihrer Eingebundenheit in gesellschaftliche Zusammenhänge betrachtet: In der modernen bürgerlichen Gesellschaft ist sie ein Lebensbereich, in dem sich Geschlechter-, Klassen- und rassistische Verhältnisse sowie weltanschauliche Vorgaben - oft konflikthaft - verschränken. Zugleich erfolgen hier Aushandlungen über die offene und Vielfalt akzeptierende Fortentwicklung der Gesellschaft.

BAND 2

AngewandTe SeXuAlWissenschaft

Herausgegeben von Ulrike Busch, Harald Stumpe,

Heinz-Jürgen Voß und Konrad Weller,

Institut für Angewandte Sexualwissenschaft

an der Hochschule Merseburg 


\author{
Manuela Tillmanns
}

\title{
Intergeschlechtlichkeit
}

Impulse für die Beratung

Psychosozial-Verlag 
Die Open-Access-Publikation wurde durch eine Förderung des Bundesministerium für Bildung und Forschung ermöglicht.

Dieses Werk ist lizenziert unter der Creative Commons Attribution-NonCommercial-NoDerivs 3.0 DE Lizenz (CC BY-NC-ND 3.0 DE). Diese Lizenz erlaubt die private Nutzung und unveränderte Weitergabe, verbietet jedoch die Bearbeitung und kommerzielle Nutzung. Weitere Informationen finden Sie unter: https://creativecommons.org/ licenses/by-nc-nd/3.0/de/

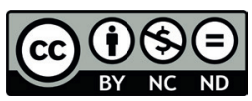

Bibliografische Information der Deutschen Nationalbibliothek Die Deutsche Nationalbibliothek verzeichnet diese Publikation in der Deutschen Nationalbibliografie; detaillierte bibliografische Daten sind im Internet über <http://dnb.d-nb.de> abrufbar.

$$
\begin{gathered}
\text { Originalausgabe } \\
\text { (C) } 2015 \text { Psychosozial-Verlag, Gießen } \\
\text { E-Mail: info@psychosozial-verlag.de } \\
\text { www.psychosozial-verlag.de }
\end{gathered}
$$

Alle Rechte vorbehalten. Kein Teil des Werkes darf in irgendeiner Form (durch Fotografie, Mikrofilm oder andere Verfahren) ohne schriftliche

Genehmigung des Verlages reproduziert oder unter Verwendung elektronischer Systeme verarbeitet, vervielfältigt oder verbreitet werden.

Lektorat: Salih AlexanderWolter

Korrektorat: Jana Motzet

Umschlagabbildung: (C) Johanna Schmitz

Umschlaggestaltung: Hanspeter Ludwig, Wetzlar

www.imaginary-world.de

Satz: metiTEC-Software, me-ti GmbH, Berlin

ISBN 978-3-8379-2493-0 (Print)

ISBN 978-3-8379-6946-7 (E-Book-PDF)

ISSN 2367-2420 (Print)

https://doi.org/10.30820/9783837969467 


\section{Inhalt}

Einleitung

Forschungsstand und gesellschaftliche Debatte

Analyse des Beratungsbedarfs

Zur Lebenssituation intergeschlechtlicher Menschen:

Aspekte wissenschaftlicher Studien

Zur Bedeutung des Peer-Ansatzes:

Selbsthilfe und Organisationen

Ausgangspunkt subjektiver Betrachtungen:

Ergebnisse qualitativer Expert_innen-Interviews

Aspekte einer ॥gerechten« Inter*-Beratung 63

Allgemeine Beratungszugänge und -formate 64

Der Ansatz psychosozialer Beratung $\quad 67$

Methoden psychosozialer Inter*-Beratung 76

$\begin{array}{ll}\text { Professionalisierung } & 80\end{array}$

Impulse an eine konkrete Umsetzungspraxis 111

Medizin vs. Beratung - eine wichtige Schnittstelle 112

$\begin{array}{ll}\text { Einstiegspforte: Onlineplattform } & 117\end{array}$ 
Das Rad nicht ständig neu erfinden:

Ausbau bestehender Strukturen

Mobile Beratung: Ein neuer Weg des Zugangs

bzw. eine Umkehr der Hemmschwelle

»Alles unter einem Dach« - Die Zukunft liegt im Aufbau

von Kompetenzzentren

Ausblick

Abkürzungsverzeichnis

Literatur und Quellen 
»Die Annahme, es gäbe nur Männer und Frauen, ist so absurd, als würde man auf dem Standpunkt verharren, die Erde wäre eine Scheibe.«

(Veith, 2012, S. 95) 


\section{Einleitung}

Am 6. Februar 2008 gewann Christiane Völling vor dem Kölner Landgericht den Prozess gegen den_die Ärzt_in ${ }^{1}$, der_die ihr im Rahmen einer Blinddarmoperation die inneren weiblichen Geschlechtsorgane entfernt hatte. Bis zu diesem Eingriff wusste Völling nichts von ihrer körperlichen Varianz « und sie wurde auch dann nicht darüber in Kenntnis gesetzt. Ihre Zustimmung zur Entfernung der inneren Geschlechtsorgane wurde nicht eingeholt; eine informierte Einwilligung wurde ihr vorenthalten. Was am Tag der Operation wirklich mit ihr geschah, erfuhr sie erst Jahre später aus ihren Krankenhausakten. Sie verklagte daraufhin den_die behandelnde_n Ärzt_in auf Schmerzensgeld und gewann (vgl. FAZ.net, 2008; Völling, 2010, S. 198). Dieser juristische Präzedenzfall führte zu einer gesteigerten öffentlichen Aufmerksamkeit für medizinische Behandlungsmethoden bei Intergeschlechtlichkeit ${ }^{2}$ (vgl. Truffer \& Seelenlos, 2011; Berendsen, 2013; Bongard, o.J.). Intergeschlechtliche Menschen unterliegen in der Bundesrepublik Deutschland medizinischen Zuweisungs- und Vereindeuti-

1 Durch das sprachliche Mittel des »Gender Gaps« (»_«) sollen explizit alle jene Identitäten angesprochen und einbezogen werden, die aus dem System der Zwei-Geschlechter-Ordnung exkludiert werden oder sich selbstbestimmt nicht verorten wollen (vgl. Herrmann, 2003, S. 22; 2007, S. 195f.). Bei Personen, die eine klare pronominale Präferenz bevorzugen, wird diese, soweit bekannt, in der vorliegenden Arbeit berücksichtigt.

2 Die weitverbreite Bezeichnung »Intersexualität« wird der Komplexität von Geschlechtlichkeit nicht in ausreichendem Maße gerecht. Deswegen wird in dieser Arbeit der Begriff »Intergeschlechtlichkeit« bevorzugt, der sich auf physische Merkmale und nicht auf (sexuelles) Begehren bezieht. Im Fokus stehen Menschen, die sich »im Hinblick auf ihre äußeren und/oder inneren Geschlechtsmerkmale und/oder ihre sogenannten Geschlechtshormone und/oder -chromosomen nicht in die medizinische Norm männlicher und weiblicher Körper einordnen lassen« (Barth et al., 2013, S. 6). 
gungspraxen, die einer Logik der Anpassung an ein $»$ Normgeschlecht $\ll^{3}$ folgen und dementsprechend Eingriffe an » uneindeutigen $\ll^{4}$ Genitalien zum Zweck der optischen Angleichung vorsehen (vgl. Barth et al., 2013, S. 7). Diese medizinische Praxis wird von den Behandelten oft als traumatisierend und gewaltvoll erlebt (vgl. Zehnder, 2011, S. 254ff.). Die ersten Anlaufstellen, die Menschen über ihre geschlechtliche Varianz unterrichten, sind in der Regel medizinische Institutionen. Ein Prozess medizinischer Diagnostik und »Behandlung « ist mehrheitlich die Folge. Obwohl mittlerweile auch von medizinischer Seite geschlechtszuweisende und -vereindeutigende Operationen zunehmend in die Kritik geraten, behält das Paradigma der $\gg$ Optimal Gender Policy $\ll^{5}$ weiterhin die $\gg$ Behandlungshoheit $\ll$.

Noch heute ist die Vorstellung verbreitet, eine stabile Identifikation mit einem der beiden Geschlechtermodelle sei als Grundbedingung psychischer Gesundheit und sozialer Einbindung anzusehen (vgl. Klöppel, 2013, S. 94). Dass diese Forschungstheorie mittlerweile überholt ist, bestätigen nicht zuletzt die Outcome-Studien ${ }^{6}$ zu den medizinischen Behandlungen Intergeschlechtlicher. Sowohl innerhalb der Studien als auch in der medizinischen Alltagspraxis ist von einem » psychosozialen Notfall « die Rede, wenn ein Kind intergeschlechtlich geboren wird (vgl. ebd.). Psychosoziale Beratungsmöglichkeiten erhalten intergeschlechtliche Menschen hingegen nur in unzureichendem Maße. Eine

3 Die essenzialistische Vorstellung eines »Normgeschlechts« (»typisch weiblich« respektive »typisch männlich«) wird in dieser Arbeit grundlegend kritisiert. Vielmehr wird davon ausgegangen, dass Geschlecht sozial konstruiert ist. In Anlehnung an Judith Butler wird hier somit der Geschlechtskörper nicht als bereits vorhanden, sondern als performativ hergestellt angesehen (vgl. Butler, 1995, S. 133). Erst durch wiederkehrende, rezitierende gesellschaftliche Prozesse und Ordnungen (vgl. Hartmann, 2004, S. 60f.) wird ein Körper geschlechtlich »gelesen, interpretiert und gewertet« (Voß, 2011, S. 14).

4 Andreas Hechler beanstandet den Terminus des »uneindeutigen « Geschlechts. Ihrer_seiner Auffassung nach ist jedes Genital eindeutig. Somit könne eine genitale Uneindeutigkeit nur in bipolar strukturierten Welt- und Gesellschaftsbildern existieren (vgl. Hechler, 2014, S. 53). Daran anschließend wird der Begriff im weiteren Verlauf der vorliegenden Arbeit in Anführungszeichen gesetzt; er verweist damit zugleich auf die Kritik am Zwei-Geschlechter-Modell.

5 Zur medizinischen Praxis der »optimalen Geschlechtszuweisung « und des Baltimorer Behandlungsprogramms von John Money und Lawson Wilkins s. den Abschnitt »Hamburger Studie zu Intersexualität« im Kapitel »Zur Lebenssituation intergeschlechtlicher Menschen: Aspekte wissenschaftlicher Studien« in dieser Arbeit oder vgl. Klöppel, 2010, S. $306 f f$.

6 Outcome-Studien werden anschließend an medizinische Eingriffe wissenschaftlich erhoben, um die Wirksamkeit des Eingriffs und dessen Auswirkung auf die Lebensqualität zu analysieren. 
Schnittstelle zwischen Medizin, Beratung und Inters ${ }^{* 7}$ existiert gegenwärtig genauso wenig wie eine professionelle, fachspezifische und psychosoziale Beratung im Sinne standardisierter Beratungsgrundlagen durch Menschen mit ausgewiesenen Beratungskompetenzen und dezidiertem Fachwissen. Vor allem in Bezug auf die Lebensqualität werden häufig Beeinträchtigungen des seelischen Wohlbefindens benannt, die den grundlegenden Bedarf beratender Strukturen im Kontext von Intergeschlechtlichkeit aufzeigen. So heißt es beispielsweise bei Katinka Schweizer und Hertha Richter-Appelt:

»Die psychische Symptombelastung, die z. B. anhand depressiver Symptome, Angst und Misstrauen erfasst wurde, entsprach bei 61\% der Befragten einem behandlungsrelevanten Leidensdruck, was bedeutet, dass hier eine psychotherapeutische Behandlung sinnvoll sein könnte. [...] Eine andere, häufig genannte Form der Unterstützung war die Teilnahme an Selbsthilfegruppen. 50\% gaben an, Erfahrungen mit Selbsthilfegruppen gemacht zu haben $[. ..] \ll$ (Schweizer \& Richter-Appelt, 2012b, S. 197).

Die Frage nach geeigneten spezialisierten Inter*-Beratungsangeboten, in Ergänzung zur Psychotherapie und den Strukturen der Selbsthilfe, ist Gegenstand der vorliegenden Arbeit. Vorrangig geht es darum, die Verbindung von Intergeschlechtlichkeit und Beratung herauszuarbeiten. Leitende Fragen sind: Wie muss eine inter*-spezifische Beratung gestaltet sein, um den Interessen, Anliegen und Erwartungen von Inters* zu entsprechen und ihren Bedürfnissen gerecht zu werden? Welche Kriterien muss sie diesbezüglich erfüllen? Wo müssen Schwerpunkte gesetzt werden und welchen Herausforderungen müssen sich die beteiligten Disziplinen stellen? Für die Entwicklung von Impulsen für die praktische Umsetzung werden vor allem die Erfahrungen und Vorstellungen von Inters* einbezogen. Diese bilden den Ausgangspunkt der konzeptionellen Überlegungen.

Zunächst werden, nach einem Überblick über die neuere Einführungs- und Forschungsliteratur, aktuelle Debatten um und Lebenslagen von Inters* auf wissenschaftlicher, subjektbezogener Ebene untersucht und im Hinblick auf die politischen Forderungen von Inters* analysiert. Dies geschieht anhand der drei

7 Die Verwendung des Sternchens (»*«) ersetzt die möglichen Zusätze »-sexuell« bzw. »-geschlechtlich« in bestimmten Komposita. Das Sternchen schafft eine Leerstelle für identitäre Diversität, bietet Raum für weitere Selbstbezeichnungen und setzt pathologisierender Sprache eine Alternative entgegen (vgl. Baumgartinger, 2008, zit. n. Lenz et al., 2012, S. 7). In dieser Arbeit werden die Begriffe »inter* «, »nter* « und »Inters* « synonym für »intergeschlechtlich«, »ntergeschlechtlichkeit« und »intergeschlechtliche Menschen« verwendet. 
zentralen deutschsprachigen Studien zur medizinischen Behandlungszufriedenheit, den Arbeitsweisen von Selbsthilfeorganisationen sowie der Analyse zweier qualitativer Interviews, die der Frage nach einer inter*-gerechten Beratung nachgehen. Eine Zusammenfassung dieser drei Blickrichtungen mündet in Betrachtungen zu einer kritisch-reflektierenden Wissenschaft.

Das Folgekapitel widmet sich dem Bereich der Beratung. Zunächst werden allgemeine Beratungszugänge vorgestellt, die im Hinblick auf Intergeschlechtlichkeit angemessene Formate darstellen, jedoch einer spezifischen Ausgestaltung bedürfen. Aufgrund der oftmals traumatischen Erfahrungen vieler Inters* wird eine psychosoziale Beratungsgrundlage als besonders geeignet angesehen. Deren Methoden müssen sich an allgemeinen beraterischen und pädagogischen Arbeitsweisen orientieren und sind im Hinblick auf Intergeschlechtlichkeit zu modifizieren. Gleichermaßen werden Überlegungen zur Professionalisierung angeregt. Exemplarisch werden drei Schwerpunktbereiche der konkreteren Analyse unterzogen, die zugleich ein wichtiges Fundament für die Beratung von intergeschlechtlichen Menschen darstellen und Möglichkeiten inter*-gerechter Vorgehensweisen abbilden. Abschließend werden die wichtigsten Grundlagen und Kriterien auf pädagogischer, sozialwissenschaftlicher und medizinischer Ebene als Anregungen für die Praxis einander gegenübergestellt und durch weiterführende Anregungen ergänzt.

Das letzte Kapitel behandelt fünf daraus resultierende Grundideen für die Entwicklung einer Inter*-Beratung, mit denen klassische Konzepte unter dem Aspekt der Lebensnähe neu überdacht werden sollen. 


\section{Forschungsstand und gesellschaftliche Debatte}

Die Zunahme an Protesten gegen die medizinische Behandlungspraxis hat seit den 1990er Jahren zu einem markanten Anstieg an biografischen Selbstzeugnissen und wissenschaftlichen Publikationen zum Thema Intergeschlechtlichkeit geführt. Viele Veröffentlichungen behandeln Inters* ausschließlich objektivierend. Es wird über sie geschrieben, anstatt sie selbst zu Wort kommen zu lassen. Aus dieser Kritik heraus wird in dieser Arbeit bewusst ein Schwerpunkt auf Publikationen von Inter*-Personen gelegt, die aus einer inneren Expert_innenposition berichten, und diese den wissenschaftlichen Veröffentlichungen von Nicht-Inters*, die aus einer äußeren Expert_innenposition berichten, gleichgestellt. Um konkrete Verbindungslinien zwischen Intergeschlechtlichkeit und professioneller Beratung zu ziehen, werden vor allem Selbstzeugnisse von »Betroffenen $\ll^{8}$ und Inter*-Aktivist_innen als wichtige und zentrale Expertisen herangezogen. Im Folgenden werden zunächst die wichtigsten Schriften und Medien vorgestellt.

Richtungsweisend für die internationale Diskussion war ein wissenschaftlicher Aufsatz von Suzanne Kessler aus dem Jahr 1990. In dem Artikel »The Medical Construction of Gender: Case Management of Intersexed Infants « wurde

8 Der Ausdruck »Betroffene« ist nicht unkritisch zu betrachten. Viele Inters* verstehen diesen Terminus im Sinne von »betroffen von einer Krankheit sein«, was zugleich eine Art Passivität impliziert, mit der sie sich nicht identifizieren können oder wollen. Im Zuge von Selbstermächtigungsprozessen werden sie selbst aktiv und verstehen Intergeschlechtlichkeit weder als Geschlechtsidentitätsstörung noch als medizinische Erkrankung, sondern als Varianz und eigenständige Geschlechtskategorie (vgl. Zehnder, 2010, S. 12). Deshalb wird der Begriff der »Betroffenheit« im Folgenden vermieden bzw. in Anführungszeichen gesetzt. 
zum ersten Mal die medizinische Behandlungspraxis an Inters* kritisiert (Kessler, 1990). Er inspirierte die Selbstorganisation der Inter*-Bewegung maßgeblich. Cheryl Chase gab dann fünf Jahre später mit ihrem_seinem autobiografischen Essay dieser Entwicklung weiteren Auftrieb (Chase, 1995).

Im deutschsprachigen Raum veröffentlichte Ulla Fröhling 2003 in Leben zwischen den Geschlechtern eine Zusammenstellung verschiedener Texte. Aus ihren jeweiligen Perspektiven berichten Inters*, Eltern und Vertreter_innen wissenschaftlicher Fachdisziplinen von ihren (traumatischen) Erlebnissen mit Mediziner_innen sowie von Selbstermächtigungskämpfen der Inter*-Bewegung und informieren über rechtliche Grundlagen. Teils als Abdruck von Interview-Formaten, teils in Essay-Form bieten die Texte einen umfassenden Querschnitt zum Themenkomplex Intergeschlechtlichkeit (Fröhling, 2003). Als eine der ersten autobiografischen Schilderungen ist das Buch Christiane Völlings Ich war Mann und Frau (2010) hervorzuheben. Völling fasste nach ihrer erfolgreichen Schadensersatzklage den Mut, ihre Geschichte niederzuschreiben und der Öffentlichkeit zugänglich zu machen. Parallel dazu erschien eine 50-minütige TVDokumentation von Britta Dombrowe mit dem Titel Tabu Intersexualität auf Arte, in der Völling ihre Erlebnisse, u. a. mit medizinischem Fachpersonal, zeigt (vgl. Dombrowe, 2010). In dieser Dokumentation wird zudem eine Familie vorgestellt und im Alltag begleitet, deren Kind Inge in einer offenen Geschlechtskategorie aufwächst. Im Kontext sehr junger Adressat_innen gelang dem Verein Intersexuelle Menschen e. V. (IMeV) 2009 mit dem Buch Lila oder was ist Intersexualität? der Versuch, Intergeschlechtlichkeit anschaulich, kindgerecht und in einfacher Sprache darzustellen (Intersexuelle Menschen e. V., 2009). Im Rahmen der Ausstellung 1-0-1 [one 'o one] intersex veröffentlichte die Initiative der Neuen Gesellschaft für Bildende Kunst 2005 einen Sammelband, der in der Diskussion um Intergeschlechtlichkeit eine zentrale Referenz darstellt (Neue Gesellschaft für Bildende Kunst, 2005). Unter Berufung auf die allgemeinen Menschenrechte wird in dieser Publikation versucht, die Anliegen intergeschlechtlicher Menschen in der Öffentlichkeit sichtbar zu machen und damit gleichstellungs- und antidiskriminierungspolitisch in einem zu agieren. Die Beiträge entstammen einer Kooperation intergeschlechtlicher und genderqueerer ${ }^{9}$ Aktivist_innen, die geschlossen die Ansicht vertreten, dass Menschenrechtsverletzungen an Inters*

9 Als queer bzw. genderqueer bezeichnen sich Menschen, die sich nicht mit der heterosexuellen zweigeschlechtlichen Matrix identifizieren können oder wollen (vgl. Perko, 2005, S. 24). Darüber hinaus verweist der Begriff »auf Handlungen und soziale Positionen, die zweigeschlechtliche und heterosexuelle Normen infrage stellen« (Time \& Franzen, 2012, S. 21). 
kein spezifisches, sondern ein gesamtgesellschaftliches Problem darstellen. Die Autor_innen gehen davon aus, dass alle Menschen von der binären Geschlechtervorstellung betroffen sind und so unter dem gesellschaftlichen Druck stehen, sich geschlechtlich eindeutig zu positionieren (vgl. Zehnder, 2010, S. 36). Der Sammelband Inter. Erfahrungen intergeschlechtlicher Menschen in der Welt der zwei Geschlechter von 2013 bietet ebenfalls eine Verknüpfung künstlerischer, politischer, solidarischer und persönlicher Texte auf internationaler Ebene. Auch diese Veröffentlichung thematisiert, wie das zuvor erwähnte Werk, Menschenrechtsverletzungen, Gewalt und Diskriminierungen gegenüber Inters* weltweit (Barth et al., 2013). Aus demselben Jahr stammt die biografische Erzählung von Clara Morgen Mein intersexuelles Kind. Weiblich männlich fließend. Aus persönlicher Perspektive schildert die Autor_in den Lebensalltag von Inter*-Familien mit seinen Hürden und benennt Schwierigkeiten im Kontext von Intergeschlechtlichkeit (Morgen, 2013).

Die im Folgenden angeführten Schriften und Medien eignen sich besonders als Einstieg für die pädagogische Bildungs- und Beratungsarbeit, da sie auf pathologisierende Determinationen aus medizinischer Perspektive verzichten und in erster Linie Lebenslagen und -realitäten von Inter*-Personen hervorheben und abbilden. Sie eignen sich darüber hinaus zur Selbstinformation. An erster Stelle möchte ich auf zwei Filmdokumentationen eingehen, die in wertschätzender und selbstbestimmter Weise Inters* und ihre persönlichen Biografien porträtieren. Es handelt sich zum einen um das »visuelle Hörstück « Die Katze wäre eher ein Vo-

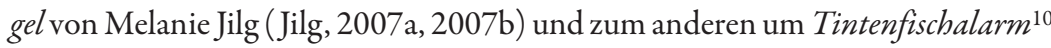
von Elisabeth Scharang (Scharang, 2006a). Letzterer Film wird im Verlauf der vorliegenden Arbeit erneut aufgegriffen. Während die beiden genannten Dokumentationen auch sequenziell für die pädagogische und für die Beratungsarbeit eingesetzt werden können, bieten sich für einen zeitlich intensiveren Einstieg der argentinische Spielfilm $X X Y^{11}$ aus dem Jahr 2007 (Puenzo, 2007) und der Roman Middlesex von Jeffrey Eugenides an (Eugenides, 2003). Beide Geschichten basieren auf fiktiven Ausgangslagen, benennen aber deutlich die Lebensrealitäten der intergeschlechtlichen Personen und die Reaktionen des sozialen und gesellschaftlichen Umfeldes. Im Hinblick auf Inter* und geschlechterreflektierende Bildungsarbeit sind die Essays von Andreas Hechler zu empfehlen, die konkrete Anregungen und Materialhinweise für die pädagogische Praxis anbieten (Hechler, 2012, 2014).

10 Siehe hierzu http://www.tintenfischalarm.at (Zugriff: 18.08.2014).

11 Siehe hierzu http://www.xxy-film.de (Zugriff: 18.08.2014). 
Der wohl bekannteste deutschsprachige Dokumentarfilm zum Thema Inter* ist Das verordnete Geschlecht ${ }^{12}$ von Oliver Tolmein und Bertram Rotermund aus dem Jahr 2001. Als inhaltliche Schwerpunkte werden dort die Themen Heteronormativität, Genitaloperationen und Geschlechterpolitik verhandelt (vgl. Hechler, 2014, S. 52; Tolmein \& Rotermund, 2014). Eine_r der Protagonist_innen ist Michel Reiter, der_die über die Dokumentation hinaus auch eine Reihe an Positionspapieren (vgl. Reiter, 2000a, 2000b, 2000c,) veröffentlicht hat, sich eindringlich für die Rechte von Inters* einsetzt und medizinische Vorgehensweisen massiv beanstandet. In einem Vortrag auf der wissenschaftlichen Fachtagung des Kongresses der European Federation of Sexology (Europäische Vereinigung für Sexologie) im Jahre 2000 in Berlin ging Reiter so weit, die »medizinischen Interventionen als Folter « zu bezeichnen und damit Begrifflichkeiten wie »chirurgische Korrekturen « oder » medizinische Interventionen « als euphemistische Verharmlosungen zu kritisieren (vgl. Reiter, 2000a). Reiter löste bei einem Großteil der anwesenden Sexualmediziner_innen Empörung aus.

Unter dem Titel Menschenrechte zwischen den Geschlechtern ist eine jüngere Analyse zum Outcome intergeschlechtlicher Menschen von Dan Christian Ghattas bekannt geworden. Diese 2013 veröffentlichte Vorstudie erhebt konkrete Daten und Aussagen zu Inter*-Lebenssituationen und nimmt im Gegensatz $\mathrm{zu}$ anderen wissenschaftlichen Studien den internationalen Bezugsrahmen in den Fokus (vgl. Ghattas, 2013), wird aber auch für eine westlich zentrierte Sicht kritisiert (Voß, 2014, S. 126).

Als weitere Quellen für die Hintergrundanalyse des Beratungsbedarfs dienten Internetforen und Weblogs, die eine vergleichsweise einfache Möglichkeit der Informationsbeschaffung, der Organisierung, Vernetzung und des Austausches darstellen - auch für die inhaltliche Orientierung ist diese Recherche sehr zu empfehlen. Unter den ausgewerteten Netzmedien befinden sich zum Beispiel das Forum vom Verein Intersexuelle Menschen e. V. (vgl. IMeV, 2010) sowie das neue Webportal insbesondere für junge trans* inter* genderqueere Menschen (vgl. meingeschlecht, o. J.), das erstmalig im Juli 2014 online ging.

Überblickt man die deutschsprachige wissenschaftliche Literatur zum Thema, sind für die letzten Jahre insbesondere fünf grundlegende Werke zu nennen:

Ulrike Klöppel schließt mit ihrer_seiner Dissertation XXOXY ungelöst eine Lücke an der Schnittstelle zwischen Medizin, Geschichte und Geschlechterforschung. Der_die Autor_in gibt einen breiten Überblick über die historischen 
Zusammenhänge der genannten Disziplinen und analysiert aus diskurstheoretischer Perspektive den gesellschaftlichen Umgang mit Intergeschlechtlichkeit ${ }^{13}$ von der Frühen Neuzeit bis heute. Dem Paradigmenwechsel im 20. Jahrhundert wird innerhalb der Studie eine besondere Aufmerksamkeit zuteil (vgl. Klöppel, 2010).

Aus einer ethnologischen Perspektive bietet Claudia Lang mittels Interviewdaten eine Orientierungshilfe, um eine größere Entscheidungs- und Handlungskompetenz auf dem Gebiet der Intergeschlechtlichkeit sicherzustellen. Der_die Autor_in gibt, indem er_sie unterschiedliche Perspektiven und Denkrichtungen einbezieht, ein vielfältiges Bild der Lebenswelt Intergeschlechtlicher, ihrer Angehörigen und der involvierten Fachdisziplinen (vgl. Lang, 2006).

Der von Katinka Schweizer und Hertha Richter-Appelt herausgegebene Sammelband Intersexualität kontrovers verhandelt aktuelle Debatten zu Intergeschlechtlichkeit in den Spektren Medizin, Ethik und Recht. Besonders interessant für Betrachtungen zur Beratungspraxis sind die dortigen Ergebnisse der IntersexStudie der gleichnamigen Hamburger Forschungsgruppe zur Behandlungszufriedenheit (vgl. Schweizer \& Richter-Appelt, 2012; Hamburger Forschergruppe Intersex, 2008; Sexualforschung Hamburg, o. J.).

Ähnlich wie Ulrike Klöppel ergründet Kathrin Zehnder in ihrer_seiner Dissertation Zwitter beim Namen nennen ebenfalls die Erfahrungen intergeschlechtlicher Menschen durch eine medizinische Behandlungspraxis (Zehnder, 2010). Als Quelle dienen Zehnder Internetforen und Weblogs. Die Ergebnisse der Untersuchung stellt er_sie im weiteren Verlauf kritisch dem medizinisch-psychologischen Diskurs gegenüber.

Im Gegensatz zu den bisher genannten Autor_innen stellt Heinz-Jürgen Voß in ihrer_seiner Interventionsschrift Intersexualität - Intersex einen deutlichen politischen Bezug her und analysiert aktuelle Debatten zu Intergeschlechtlichkeit. Er_sie fordert eine stärkere Einbeziehung der Interessen und Forderungen von Inter*-Bewegungen (Voß, 2012a).

Keine dieser Publikationen zu Intergeschlechtlichkeit thematisiert explizit Beratung.

Einen guten Einblick in den Bereich Beratung im Allgemeinen bietet das von Frank Engel, Frank Nestmann und Ursel Sickendiek herausgegebene zwei-

13 Allerdings kann das Konzept Intergeschlechtlichkeit bzw. Intersexualität historisch selbstverständlich nicht einfach rückübertragen werden. Der Begriff Intersexualität/Intersex wurde erst zu Beginn des 20. Jahrhunderts geprägt und erst seit der europäischen Moderne entwickelte sich nach und nach der medizinisierte Blick. Klöppels empfehlenswerte Arbeit weist begriffliche und historische Sensibilität auf. 
bändige Handbuch der Beratung. Sowohl das Werk insgesamt als auch der von den Herausgeber_innen verfasste einführende Beitrag »Beratung « zu sozialpädagogischen und psychosozialen Beratungsansätzen informieren umfassend und facettenreich über die Grundlagen und die Komplexität von Beratung, inklusive ihren Methoden, Disziplinen und Zugängen. Im Handbuch findet sich auch ein sehr differenziertes Kapitel zum Themenkomplex Geschlecht und Beratung (vgl. Vogt, 2004; Stecklina \& Böhnisch, 2004; Tatschmurat, 2004; Wiesendanger, 2004). 


\section{Analyse des Beratungsbedarfs}

Individuelle »Betroffenheiten « werden in wissenschaftlichen Ausarbeitungen oft nur unzureichend bzw. am Rande diskutiert. Inters* und Interessierte erleben wissenschaftliche Facharbeiten, im Vergleich mit persönlichen Erfahrungsberichten, oft als realitätsfern, emotional distanziert und wenig kritisch. Bei der Beschäftigung mit Selbstzeugnissen fällt es dagegen leichter, sich mit »Betroffenen « zu identifizieren, als dies durch das Lesen akademischer Studien möglich ist. Dennoch sind wissenschaftliche Erhebungen als Methode wichtig. Sie erlauben es, Lebenslagen und Erfahrungshintergründe vergleichend zu untersuchen. Öffentliche Debatten von Inters* werden mit wissenschaftlichen Argumenten untermauert, aber auch reflektiert. Hieran anschließend ist hinsichtlich der vorliegenden Untersuchung zu fragen, wie Beratung ${ }^{14}$ inhaltlich aufgebaut und praktisch umgesetzt werden muss, um den Bedürfnissen der Zielgruppe intergeschlechtlicher Menschen gerecht zu werden. Bislang existieren noch keine Studien zu professioneller Beratung, die klare Anregungen und Erwartungen formulieren. Deshalb werden in dieser Arbeit Erfahrungsberichte intergeschlechtlicher Menschen mit wissenschaftlichen Analysen zusammengeführt, um zunächst die konkreten Beratungsbedarfe aufzuzeigen. Das geschieht, um die Beratungsinstrumente zielgruppenspezifisch auf die Anforderungen von Inters* abzustimmen und gleichzeitig Vorschläge für die Neu- und Weiterentwicklung von Beratungsangeboten aufzustellen, sodass Intergeschlechtlichkeit adäquat berücksichtigt wird.

Im Folgenden wird ein Überblick über existierende Beschreibungen von persönlichen Erfahrungen Intergeschlechtlicher und die wissenschaftlichen For-

14 Beratung wird hier als Prozess professioneller Unterstützung bei der Problemlösung und Identitätsarbeit verstanden (vgl. Stark, 2004, S. 535). 
schungsergebnisse gegeben. Es schließt sich eine inhaltliche Analyse wissenschaftlicher Studien an, die den deutschsprachigen medizinischen Diskurs zentral bestimmen. Der medizinischen Perspektive wird als Gegengewicht die Expertise von Inters* unter dem Gesichtspunkt des »Peer-Involvements « ${ }^{15}$ gegenübergestellt. Die Entwicklung und Bedeutung von Selbsthilfe wird anhand dreier zentraler Inters*-Organisationen aus dem deutschsprachigen Raum veranschaulicht. Darüber hinaus werden deren Forderungen zum politischen und gesellschaftlichen Umgang mit Intergeschlechtlichkeit dargelegt. Abschließende Bemerkungen zum allgemeinen Beratungsbedarf von Inters* stellen die Ergebnisse von Expert_innen-Interviews vor. Diese bieten Ansätze und Anregungen für eine geeignete und umfassende Inter*-Beratung.

\section{Zur Lebenssituation intergeschlechtlicher Menschen: Aspekte wissenschaftlicher Studien}

Obwohl das Thema Intergeschlechtlichkeit in den letzten zehn Jahren Einzug in die deutschsprachige Forschungslandschaft gehalten hat, fehlen noch immer wissenschaftlich-repräsentative Langzeitstudien, die einen Ein- und Überblick zu den Lebenslagen intergeschlechtlicher Menschen liefern (vgl. Wunder, 2012, S. 36; Schweizer \& Richter-Appelt, 2012b, S. 188). Zur Erhebung der Lebensqualität gehören demnach die Betrachtungen zur physischen und psychischen Gesundheit, zur Behandlungszufriedenheit, zu Einstellungen hinsichtlich medizinischer Interventionen und ihren möglichen Folgen sowie den Wünschen und Forderungen in Bezug auf zukünftige Behandlungsverfahren. In diesem Zusammenhang werden im Folgenden die bislang einflussreichsten deutschsprachigen Studien ${ }^{16}$ auf ihre Inhalte analysiert, ihre Verfahren erörtert und die wichtigsten Ergebnisse vorgestellt. Weder die Studie des Netzwerks DSD noch die Hamburger Intersex-Studie oder die Erhebung des Deutschen Ethikrates entsprechen

15 Unter dem Begriff des »Peer-Involvements « werden in unterschiedlichen Kontexten und vielfältigen Formen »Peers« (Gleiche) als Träger_innen personalkommunikativer Botschaften eingesetzt (vgl. Backes, 2003, S. 176). Formen und Ziele von »Peer-Support« werden im weiteren Verlauf dieser Arbeit eingehender beleuchtet.

16 Die hier analysierten Studien verweisen auf den Bezug zum dominanten medialen Diskurs im deutschsprachigen Raum. Andere dieser sogenannten Outcome-Studien werden innerhalb dieses Diskurses nur randständig behandelt. Ergebnisse internationaler Studien zu anatomischen und funktionalen Zufriedenheiten infolge medizinischer Eingriffe sind bei Voß nachzulesen (vgl. Voß, 2012a, S. 50-55). 
dabei gänzlich den Kriterien der Repräsentativität. Alle drei Studien weisen die Gemeinsamkeit auf, dass Aussagen sehr junger Proband_innen nur unter Vorbehalt in der wissenschaftlichen Analyse betrachtet werden können. Offenkundig ist, dass Eltern entweder stellvertretend oder in Zusammenarbeit mit ihren Kindern auf die Fragen geantwortet haben (vgl. Wunder, 2012, S. 37; Bora, 2012, S. 8). Das bedeutet im Umkehrschluss, dass die gemachten Aussagen auch nicht den Minderjährigen zugerechnet werden können. Die Stellvertreter_innen-Entscheidung durch die Eltern wird ebenfalls in allen drei vorliegenden empirischen Untersuchungen als $\gg$ formal gegeben angesehen « (Wunder, 2012, S. 37). Lediglich »die Art und Qualität der Aufklärung « (ebd.) werden als nicht ausreichend beschrieben. So heißt es bei Wunder konkret, Entscheidungsdruck, fehlende Alternativen und das Nicht-Einbeziehen der »Betroffenen « würden bemängelt (vgl. ebd.).

\section{Studie des Netzwerks DSD ${ }^{17} /$ Intersexualität}

Das Netzwerk DSD/Intersexualität ist ein Zusammenschluss von Wissenschaftler_innen diverser Fachdisziplinen (Medizin, Biologie, Sozialwissenschaften), Ärzt_innen, Elterninitiativen und Selbsthilfeorganisationen. Seit 2003 wird es vom Bundesministerium für Bildung und Forschung (BMBF) finanziert (vgl. Kleinemeier/Jürgensen, o. J., S. 4). Als eines der wichtigsten Projekte dieses Netzwerks gilt die »klinische Evaluationsstudie «, die in den Jahren 2005 bis $2008^{18}$

17 Seit der Chicago Consensus Conference von 2005 hat sich vor allem im medizinischwissenschaftlichen Diskurs der Terminus »Störungen der Geschlechtsentwicklung (engl.: disorders of sex development, DSD) durchgesetzt (vgl. Schweizer \& Richter-Appelt, 2012a, S. 109; Schweizer, 2012, S. 26f.). Anlässlich dieser DSD-Nomenklatur regte sich vor allem in Kreisen von Inter*-Verbänden und -Aktivist_innen Widerstand (vgl. Schweizer, 2012, S. 26f.). Aufgrund des stark pathologisierenden Charakters wird dieser Terminus sowohl von der bundesdeutschen Vertretung der Internationalen Vereinigung Intergeschlechtlicher Menschen (IVIM) als auch von der Organisation Intersex International (OII) abgelehnt (vgl. Oll Deutschland, o.J.) Zudem ist er auch in Wissenschaftskreisen umstritten (vgl. Klöppel, 2010, S. 22). Zur Vermeidung des Störungsbegriffs sei alternativ die Rede von »Differenzen der Geschlechtsentwicklung" (vgl. Schweizer, 2012, S. 26f.). Dieser Haltung schließt sich auch der Deutsche Ethikrat in seiner Stellungnahme an (vgl. Deutscher Ethikrat, 2012a, S. 12).

18 Im Bericht der ersten Ergebnisse ist ein Projektzeitraum von 2005 bis 2007 angegeben, im weiteren Verlauf des Textes wird jedoch davon berichtet, dass die letzten Befragungen 2008 stattfanden, weshalb sich die hiesigen Ausarbeitungen auf diese Zeitangabe beziehen. 
durchgeführt wurde (vgl. ebd.). Insgesamt wurden 439 Personen befragt. Sowohl »Betroffene « selbst als auch Eltern intergeschlechtlicher Kinder partizipierten an der Studie. Drei Viertel (329) der Befragten waren minderjährig, ein Viertel (110) im Erwachsenenalter (vgl. Wunder, 2012, S. 37). Zum Zeitpunkt ihrer Entstehung galt die Studie damit als größte Befragung zu Intergeschlechtlichkeit im deutschsprachigen Raum (vgl. Kleinemeier \& Jürgensen, o. J., S. 10). Ausgangslage und Hintergrund für diese Studie war der sich auch im medizinischen Diskurs durch die Kritik am »Optimal Gender Paradigma « wandelnde Umgang mit Intergeschlechtlichkeit:

»Es steht nicht mehr immer das möglichst optimale Erscheinungsbild eines Menschen im Vordergrund, sondern die möglichst große Lebenszufriedenheit und ein hohes Ausmaß an Gesundheit « (Kleinemeier \& Jürgensen, o.J., S. 6).

Weltweit erstmalig wurden Befragungen zu unterschiedlichen Aspekten des Lebens von Inters* aller Altersstufen durchgeführt. Das Erkenntnisinteresse der Studie galt dem besseren Verständnis der Lebenssituation von Inters*, dem Aufzeigen spezifischer Problemfelder und der Entwicklung adäquater Behandlungs- und Lösungsstrategien (vgl. ebd., S. 10). Das Hauptaugenmerk lag auf der psychischen, physischen und sozialen Situation von Inters* und ihren Familien. Vor dem Hintergrund medizinischer Behandlung und Betreuung sollten bisherige Erfahrungen in diesem Kontext subjektiv eingeschätzt und bewertet werden (vgl. ebd., S. 6f.). Dabei war es ein Anliegen der Studiengruppe, sowohl die Vergangenheit als auch die aktuelle Lebenssituation der Inters* in Form gesicherter Erkenntnisse zu erfassen, um daraus ein einheitliches Handlungsrepertoire für die aktuelle und zukünftige Praxis zu entwickeln (vgl. ebd., S. 7). Da die abgeschlossenen Gesamtforschungsergebnisse bislang nicht veröffentlicht wurden (vgl. Wunder, 2012, S. 37), beziehen sich die vorliegenden Ausarbeitungen auf die ersten Zwischenergebnisse, die in einem Bericht für Studienteilnehmende und deren Eltern zusammengetragen wurden (Kleinemeier \& Jürgensen, o.J.).

Nicht nur bezüglich Intergeschlechtlichkeit gilt: Medizinische Behandlungserfahrungen stehen oft im Zusammenhang mit Traumatisierungen. Ein erneutes Abfragen der Erlebnisse kann bei den teilnehmenden Personen retraumatisierend wirken. Um dies zu vermeiden, wurden die Fragebogen nicht per Post versandt, stattdessen erfolgte die Erhebung dezentral in vier verschiedenen Studienzentren in Deutschland, Österreich und der Schweiz, unter Leitung des Klinikums für Kinder- und Jugendmedizin in Lübeck (vgl. ebd., S. 4). Ziel dessen war, dass 
erfahrene Psycholog_innen den Teilnehmenden für Fragen und Anliegen persönlich zur Verfügung standen (vgl. ebd., S. 7). ${ }^{19}$

Um ein möglichst ganzheitliches Forschungsergebnis zu erzielen, wurden unterschiedliche Fragebogen konzipiert, die inhaltlich auf die jeweilige Altersgruppe und die damit einhergehenden Entwicklungsphasen abgestimmt waren. Das bedeutet einerseits, dass mit zunehmendem Alter des Kindes die Komplexität der Fragen steigt. Andererseits erlaubt dieses Vorgehen den Rückschluss, dass eine medizinische Behandlung im Kindesalter Einfluss und Auswirkungen auf weitere Lebensphasen hat. ${ }^{20}$ In Zusammenarbeit mit Selbsthilfegruppen und medizinisch-psychologischen Expert_innen entstanden insgesamt vier Fragebogen: für Eltern von intergeschlechtlichen Kindern - auszufüllen je nach Alter der Kinder 1) ohne oder 2) mit deren Beteiligung; 3) für Jugendliche und 4) für erwachsene Inters* (vgl. ebd., S. 7f.).

Trotz der Problematisierung der Bezeichnung DSD durch Inter*-Selbstorganisationen hat sich die Studienleitung dazu entschlossen, diese Begrifflichkeit beizubehalten, aber anstelle von »Störungen « eher von »Besonderheiten « der Geschlechtsentwicklung zu sprechen (vgl. ebd., S. 4f.). Möglicherweise führte gerade diese Auseinandersetzung zur Begrifflichkeit dazu, dass in der Studie auch nach der Selbstbezeichnung der Inters* bzw. nach den Benennungen der Eltern gefragt wurde. Anders als in den anderen beiden Untersuchungen werden in der Netzwerkstudie sowohl persönliche Vorlieben der Teilnehmenden als auch Kritik an der begrifflichen Benennung erforscht. Jedoch erwähnt der Bericht von Martina Kleinemeier und Martina Jürgensen nicht, ob dabei Kriterien zur Auswahl gestellt wurden oder ob es sich um eine offene Fragestellung handelte. Die von den erwachsenen Inters* genannten Begriffe sind in Reihenfolge der Häufigkeit ihrer Nennung: Krankheit (25,5\%), Intersexualität (17,3\%), besondere Geschlechtsentwicklung (12,7\%). Auch nutzten Inters* die konkrete medizinische Diagnose oder eine eigene Bezeichnung wie z. B. Genitalveränderung, Zwitter und Hormonstörung (vgl. ebd., S. 5f.). Die Hälfte der Jugendlichen machte allerdings keine Angaben zu einem bevorzugten Begriff, was darauf zurückzuführen sein könnte, dass sie für sich selbst noch keine Eigenbezeichnung gefunden haben und/oder insgesamt eher wenig über ihre Intergeschlechtlichkeit sprechen (vgl. ebd., S. 6). Über 80 Prozent aller befragten Personen waren

19 Die Frage, wie die Projektbogen an die jeweiligen Proband_innen verteilt wurden und ob diese zu Hause oder in einem der Studienzentren ausgefüllt wurden, wird im Bericht selbst nicht geklärt.

20 Rückschlüsse werden allerdings schon durch das Vorgehen vorstrukturiert. Unterschiedliche Fragen können bereits für große Differenzen bei den Ergebnissen sorgen. 
bis zum Zeitpunkt der Erhebung mindestens einmal geschlechtszuweisend und geschlechtsvereindeutigend operiert worden. Das Aufkommen von Genitaloperationen steigt kongruent zum Alter. Ein Großteil der Eingriffe (86\%) wurde bereits bis zum Schulantritt durchgeführt (vgl. ebd., S. 16). Ein Viertel der Teilnehmenden (25\%) berichtet von Folgebeschwerden, darunter insbesondere Fistelbildungen (40,5\%), Harnwegsinfekte (32\%), Stenosen (Harnwegsverengungen) (27\%) und Miktionsbeschwerden (Probleme beim Urinieren) (21,4\%) (vgl. ebd., S. 17).

Gemäß der Erhebung sind die Eltern der Inters* vor allem unzufrieden mit der Mitteilung der Diagnose und dem Informationsmanagement der behandelnden Ärzt_innen. Sie kritisieren insbesondere die Dauer des diagnostischen Prozesses, den Umgang mit der emotionalen Betroffenheit der Eltern sowie generell die Vermittlung von Informationen zu Behandlungsmöglichkeiten und Medikamenten (vgl. ebd., S. 18). Die für die erwachsenen Inters* erhobenen Werte im Hinblick auf die allgemeine Behandlungszufriedenheit liegt noch unterhalb des Durchschnittswerts für alle Inters* (vgl. ebd.). Häufig steht diese Unzufriedenheit in Verbindung mit der Suche nach kompetentem medizinischem Fachpersonal (vgl. ebd., S. 19). Nur bei etwa einem Drittel (34\%) der medizinisch behandelten Inters* (Minderjährige und Erwachsene) bzw. deren Eltern wurden durch die Ärzt_innen auch Kontakte zu Selbsthilfegruppen vermittelt (vgl. ebd.), wobei nur ca. 20 Prozent der Informierten den Kontakt auch aufnahmen (vgl. ebd., S. 20). Mögliche Gründe oder Hemmschwellen diesbezüglich werden nicht benannt.

Der Fragenkomplex zur gesundheitsbezogenen Lebensqualität umfasste Fragen zum körperlichen und psychischen Wohlbefinden, zum Selbstwert und dem sozialen Bezugsrahmen (Familie, Freunde, Kindergarten, Schule). Eltern sahen bei ihren Inter*-Kindern Beeinträchtigungen der Lebensqualität insbesondere im psychischen Bereich; demgegenüber gaben die befragten Kinder und Jugendlichen eher Einschränkungen für den Bezugsrahmen (insbesondere bezüglich der Familie) und das eigene Körpererleben an. Einig sind sich Eltern und Kinder/Jugendliche in ihrer Einschätzung, dass die Lebensqualität intergeschlechtlicher Personen mit steigendem Alter abnehme. Die Studienleiter_innen führen diese Annahme darauf zurück, »dass die Jugendlichen mit DSD eine Vielzahl an Entwicklungsaufgaben zu lösen haben, deren Lösung durch die DSD erschwert werden kann « (ebd., S. 21). In 45 Prozent der Fälle bestätigen die befragten Personen »behandlungsrelevante psychische Symptome wie Depression, Angst und reaktive Störungen « (Wunder, 2012, S. 37f.; Kleinemeier \& Jürgensen, o. J., S. 24). Dies belegt auch folgende Aussage aus dem Bericht: 
»Die Geburt eines Kindes mit einer besonderen Geschlechtsentwicklung ist für Eltern in der Regel ein unerwartetes Ereignis. Auch die spätere Diagnose in der Kindheit oder während der Pubertät wird sowohl von den Kindern und Jugendlichen selber als auch von den Eltern als belastendes Ereignis beschrieben. Aus Berichten von heute Erwachsenen ist bekannt, dass sie während ihrer Kindheit unter dem Gefühl des »Anders-Seins « gelitten [hätten] und vermehrt Depressionen und soziale Ängste entwickelten, was zu verschiedenen Verhaltensauffälligkeiten geführt habe (z. B. sozialer Rückzug) « (Kleinemeier \& Jürgensen, o. J., S. 22).

Geschlechtsidentität und Geschlechterrollenverhalten werden in dieser Studie zwar mit der psychosexuellen Entwicklung des Menschen gleichgesetzt, allerdings sind einige Aussagen der Autor_innen in ihrer Konsequenz nicht abschließend durchdacht. So heißt es zu Beginn der Studie, dass im Arbeitsparadigma der Forschungsgruppe das äußere Erscheinungsbild der Genitalien weniger wichtig sei als ein hohes Maß an Lebensqualität und Gesundheit (vgl. ebd., S. 6). Dennoch wird von der Forschungsgruppe bezüglich der Geschlechtsidentität der ein Defizit suggerierende Begriff der »Störung « verwendet:

»Wenn sich Menschen mit einem biologisch eindeutig weiblichen Körper als Männer fühlen (und umgekehrt), wird von einer Geschlechtsidentitätsstörung gesprochen. Hierbei handelt es sich um eine psychische Störung « (Kleinemeier \& Jürgensen, o.J., S. 25).

Weiterhin führen die Autor_innen aus:

»Da bei Menschen mit DSD der Körper schon von der Definition her nicht eindeutig männlich oder weiblich ist, kann bei ihnen auch keine wirkliche Geschlechtsidentitätsstörung vorliegen « (ebd.).

Auch wenn, wie es auf den ersten Blick scheint, eine positive Absicht hinter dieser Aussage steckt, muss von der Verwendung des Begriffes »Geschlechtsidentitätsstörung « aufgrund seiner diffamierenden und pathologisierenden Wirkung abgesehen werden. In der logischen Weiterführung des Gedankens wird diese Position am Beispiel von Trans*-Menschen veranschaulicht. Denn Trans* wird nach wie vor in der internationalen statistischen Klassifikation der Krankheiten und verwandter Gesundheitsprobleme (ICD-10) als » psychische Störung « eingeordnet und aufgeführt. Dieselbe Argumentation und Kritik greift hinsichtlich der Bezeichnung und des besonderen Fokus auf vermeintlich 
»geschlechtsuntypisches « Verhalten. Die Autor_innen nutzen darüber hinaus Attribute, die klassische Rollenstereotype (»typisch weiblich « respektive »typisch männlich «) bedienen und reproduzieren (vgl. ebd.). Sie greifen zudem auf die Untersuchungsmethode der Spielzeugpräferenz zurück, mit der das Spielverhalten der teilnehmenden Kinder von » sehr typisch mädchenhaft « über »neutral « bis hin zu »sehr typisch jungenhaft « skaliert wird (vgl. ebd., S. 26). Ebenso wurden die Eltern dazu aufgefordert, das Geschlechterrollenverhalten ihrer Kinder in bestimmten Situationen anzugeben und zuzuordnen, in welchem Ausmaß sich das Kind besonders weiblich oder besonders männlich verhalte (vgl. ebd., S. 27):

»Darüber hinaus wird das Ausmaß des gegengeschlechtlichen Verhaltens und der gegengeschlechtlichen Einstellungen berechnet. Auf diese Weise soll untersucht werden, ob es bei den Kindern Hinweise auf etwaige Unsicherheiten hinsichtlich ihrer Identifikation als Mädchen oder Junge gibt « (ebd.).

Geschlechtliche Identität wird in der Studie lediglich binär gedacht, da von einem »männlich/weiblich «- respektive »typisch/untypisch «-Vorverständnis hinsichtlich Berufswahl und Rollenkodex ausgegangen wird. Das bestätigt auch die Aussage bezüglich der Gruppe der Jugendlichen:

»Nach langen Überlegungen im Vorfeld der Studie haben wir beschlossen, Jugendliche (und Erwachsene) nicht nach dem Geschlechtsrollenverhalten zu befragen, sondern uns auf Fragen zur Geschlechtsidentität zu beschränken, da es unserer Ansicht nach heutzutage weder außergewöhnlich noch relevant ist, wenn Menschen einen geschlechtsuntypischen Beruf wählen oder allgemein ein eher untypisches Rollenverhalten zeigen « (Kleinemeier \& Jürgensen, o.J., S. 28).

Mit den Fragen zu Partner_innenschaft und Sexualität erhoben die Autor_innen der Studie signifikante Unterschiede zwischen den Inters* und der Vergleichsgruppe (ohne intergeschlechtliche Varianz). Obwohl Inter*-Jugendliche sich in ihrer grundsätzlichen Einstellung zu Sexualität nicht von anderen Jugendlichen unterscheiden, geben drei Viertel der Befragten an, trotz des Wunsches nach einer festen Partner_innenschaft ungewollt Single zu sein und nur sehr wenig praktische sexuelle Erfahrungen - Masturbation (ca. 75\%), Petting (75\%), Geschlechtsverkehr (90\%) - zu haben (vgl. ebd., S. 30). Auch die erwachsenen Inters* berichten öfter als die Vergleichsgruppe von ungewollter Partner_innenlosigkeit und fehlender sexueller Aktivität. Sie geben in größerem Maße an 
(35\%), Schwierigkeiten zu haben, sexuelle Kontakte zu knüpfen. Zudem berichten 40 Prozent davon, sich sexuell lustlos zu fühlen und benennen Schmerzen bzw. die Angst vor Schmerzen bei sexuellen Kontakten. Die Unsicherheiten stehen in deutlichem Zusammenhang mit den chirurgischen Eingriffen (vgl. ebd., S. 31).

Innerhalb der Studie wurden Angaben von Kindern zu ihrer Situation ab dem Zeitpunkt ihrer Geburt berücksichtigt. Dies lässt Rückschlüsse darauf zu, dass auch gegenwärtig noch am Behandlungsprogramm der 1950er Jahre festgehalten wird, obgleich die Stichprobe des Ethikrates aufweist, dass »die meisten [...] befragten Mediziner und Medizinerinnen [über] einen Wandel in der Grundeinstellung und eine größere Zurückhaltung bei frühen Eingriffen berichtet haben « (Wunder, 2012, S. 37).

Insgesamt zeigen die Ergebnisse der Netzwerkstudie eine hohe Übereinstimmung von Eltern und behandelnden Ärzt_innen hinsichtlich der Notwendigkeit einer zeitnahen Aufklärung der Kinder über ihre Intergeschlechtlichkeit (vgl. Kleinemeier \& Jürgensen, o.J., S. 39). So gehen die Autor_innen sogar davon aus, dass eine frühzeitige, altersgemäße Aufklärung die Chance bietet, Kinder darin zu unterstützen, ihre »Besonderheit « als Teil von sich zu verstehen und anzunehmen (vgl. ebd., S. 36). Die Frage nach dem richtigen Umgang mit der Intergeschlechtlichkeit im sozialen Umfeld bewegt fast alle »Betroffenen «. Während Eltern oft den engeren familiären Bezugsrahmen und Freundeskreis mit einbeziehen, sind Jugendliche ihrerseits zurückhaltender. Als Begründung wird die Angst angeführt, auf Unverständnis und Ablehnung zu stoßen (vgl. ebd., S. 39f.).

Ein wesentlicher Kritikpunkt an der Netzwerkstudie ist die genaue Differenzierung zwischen den einzelnen medizinischen Diagnoseformen von Intergeschlechtlichkeit. Daran schließt die auch immer wiederkehrende Überprüfung auf binäre Rollenmuster an (so z. B. bei der Untersuchung der Spielzeugpräferenz). In der Studie bleibt ein hegemoniales Geschlechterverständnis zentral und wird kaum hinterfragt. So wird ein kohärenteres geschlechtliches Auftreten als »weiblich « oder » männlich « als gleichbedeutend mit mehr Lebensqualität und weniger Andersartigkeit gesetzt. Sexual- und sozialwissenschaftliche Sichtweisen und Theorien zur Geschlechtsidentität sowie die Annahme, dass Geschlechtlichkeit multifaktoriellen Einflüssen unterliegt (zum Beispiel Sozialisation), werden in der Studie außer Acht gelassen. Obwohl Selbsthilfe- und Interessenverbände im Netzwerk mitgewirkt haben und bipolare Geschlechterdiskurse kritisierten, wurde innerhalb des Forschungsprojekts auf entsprechende Geschlechterkonstruktionen zurückgegriffen. 


\section{Hamburger Studie zu Intersexualität}

Initiator_innen und Durchführende der Hamburger Intersex-Studie waren die Mitarbeiter_innen der Forscher_innengruppe Intersexualität des Instituts für Sexualforschung und Forensische Psychiatrie am Universitätsklinikum HamburgEppendorf (UKE) unter der Leitung von Hertha Richter-Appelt und Katinka Schweizer. Ziel der Untersuchung war eine umfassende Erhebung der Behandlungserfahrungen und zentraler Aspekte der Lebensqualität von erwachsenen Inters*. Den Hintergrund der Studie bildete die aufkeimende öffentliche Kritik an der vorherrschenden Behandlungspraxis, speziell am Paradigma der $\gg$ Optimal Gender Policy $\ll^{21}$ - vor allem auch durch ehemalige Patient_innen (vgl. Schweizer \& Richter-Appelt, 2012b, S. 187f.). Als Grundannahme geben die Herausgeber_innen an:

»Intersexualität stellt für Betroffene und ihre Familien eine ungewöhnliche, komplexe und herausfordernde Lebenserfahrung dar. Betroffene sind aufgrund der untypischen somatosexuellen und gegebenenfalls auch psychosexuellen Entwicklung Erfahrungen der Differenz, des Anders-Seins ausgesetzt, die sich körperlich sichtbar v.a. im Bereich der Genitalien abspielen und damit besonderer Tabuisierung unterliegen können « (Schweizer \& Richter-Appelt, 2012b, S. 188).

Neben der Erforschung von Behandlungserfahrungen und Lebensqualität richtete sich die Studie auf die Wege der individuellen Bewältigung traumatisierender

21 Die Praxis der »optimalen Geschlechtszuschreibung « wurde in den 1950er Jahren zur Routinebehandlung. Durch Forschungen des amerikanischen Psychologen John Money und des Kinderarztes Lawson Wilkins am John Hopkins Hospital in Baltimore vorangetrieben, wurde dieses Vorgehen unter der Bezeichnung Baltimorer Behandlungsprogramm international bekannt (vgl. Wunder, 2012, S. 36; Klöppel, 2013, S. 92). Während Wilkins aufgrund des äußeren Erscheinungsbildes systematisch genitale Operationen an intergeschlechtlichen Kindern durchführte, erforschte Money im Anschluss an diese Eingriffe, ob sich die »behandelten « Intergeschlechtlichen mit ihrer zugeschriebenen »Geschlechtsrolle identifizieren, ein angepasstes Verhalten und heterosexuelle Orientierung zeigten, und zwar selbst dann, wenn die Zuweisung nicht mit dem biologischen Geschlecht übereinstimmte« (Klöppel, 2013, S. 93). Moneys Untersuchungen kamen zu dem Ergebnis, dass »Genitaloperationen an intersexuellen Kindern als eine planmäßige Steuerung der Psychosexualität begründet werden« könnten (vgl. ebd.). Somit wurde das Baltimorer Behandlungsprogramm von Wilkins mit der Theorie der sozialen Prägung der Psychosexualität von Money als Interaktionstheorie wissenschaftlich verankert. Diese Vorstellungen führten zu der Annahme, »dass die psychosexuelle Entwicklung und damit die Geschlechtsidentität aus der Einheit des Geschlechts herausgelöst und kontrolliert werden [könne]« (ebd., S. 94). 
Erfahrungen und auf die Fragen, ob und wenn ja, welche Coping-Strategien 22 intergeschlechtliche Menschen anwenden, welche Aussagen sie zu ihrem Identitätsund Geschlechtserleben treffen und inwiefern Besonderheiten in der körperlichen und psychischen Gesundheit und Entwicklung vorliegen (vgl. ebd.). In den Jahren 2007 bis 2008 wurden dahingehend 69 jugendliche und erwachsene intergeschlechtliche Personen im Alter von 16 bis 60 Jahren für die Studie befragt (vgl. Wunder, 2012, S. 37). Die Wege zur Teilnahme an der Erhebung waren dabei sehr vielfältig: Inters* und ihre Angehörigen wurden durch behandelnde Ärzt_innen, über die Homepage des Projekts, durch Selbsthilfegruppen, spezifische Veranstaltungen und Kongresse oder Infomaterial informiert und als Teilnehmer_innen gewonnen (vgl. Schweizer \& Richter-Appelt, 2012b, S. 193).

Das Studienvorhaben unterlag einer komplexen und vielschichtigen Planung. Um eine möglichst ganzheitliche Betrachtung zu erwirken, wurden sowohl quantitative als auch qualitative Daten erhoben und dabei standardisierte Methoden mit offenen Fragen kombiniert. Mittels Fragebogen wurden fünf wesentliche Themen erfasst. Erfragt wurden neben den klassischen (1) soziodemografischen Angaben auch (2) individuelle Erfahrungen und die Zufriedenheit mit der körperlichen Entwicklung, (3) mit Aufklärung und Information, Diagnostik, Medizin und Behandlung sowie hinsichtlich (4) der psychischen Symptombelastung und dem Verhältnis Körper, Geschlecht und Identität zueinander. Aber auch (5) Fragen zur allgemeinen Lebenszufriedenheit sowie zu Sexualität und Partner_innenschaft waren enthalten (vgl. ebd., S. 191).Zum gesamten Fragebogen konnten die Teilnehmenden individuelle Anmerkungen und Kommentare anfügen. Im Unterschied zu den anderen hier erwähnten Studien wurde die Erhebung weder postalisch noch dezentral durchgeführt, sondern zentral im Hamburger Institut. Dahinter stand die Überlegung, eventuell auftretende Krisen in der Auseinandersetzung mit den eigenen Erinnerungen direkt auffangen zu können. So wurde jeder Fragebogen durch ein persönliches psychologisches Fachgespräch ergänzt. Nach Bedarf und nur mit ausdrücklicher Einwilligung der Proband_innen bestand die Möglichkeit, medizinische Akten aktueller oder ehemaliger Ärzt_innen anzufordern und gegebenenfalls Behandlungsmaßnahmen in Bezug auf die Diagnosen zu validieren (vgl. ebd.).

Die Ergebnisse dieser Studie bildeten weitgehend ihre Vorannahmen ab und bestätigten, dass Intergeschlechtlichkeit bei einem Großteil der Teilnehmen-

22 Die Bezeichnung Coping-Strategie kommt aus der Resilienzforschung (Forschung zu Belastbarkeit/Widerstandsfähigkeit) und beinhaltet im Wesentlichen Strategien zur Bewältigung von beispielsweise Lebenskrisen und/oder Gewalterfahrungen. 
den erhebliche Auswirkungen auf Lebensrealität und Lebenslage hat. Bereits in der Zwischenauswertung sind bei ca. 60 Prozent der Gesamtgruppe auffällige psychische Belastungen zu verzeichnen. So werden Suizidalität (61\%) und Selbstverletzungserfahrungen explizit benannt, die im selben Maße den Prävalenzen einer sexuell und physisch traumatisierten Vergleichsgruppe entsprachen (vgl. Schützmann et al., 2009, zit. n. Schweizer \& Richter-Appelt, 2012b, S. 189). Ebenso weisen die Ergebnisse der Endauswertung eine erhöhte Beeinträchtigung des subjektiven Körperempfindens (vgl. Brunner et al., 2012, zit. n. Schweizer \& Richter-Appelt, 2012b, S. 190) und der sexuellen Lebensqualität auf, die oft in Zusammenhang mit erlebten chirurgischen Eingriffen steht (vgl. Schönbucher et al. 2010, zit. n. Schweizer \& Richter-Appelt, 2012b, S. 190). So versucht diese Studie auch die Verarbeitung des Erlebten und die Auswirkungen der Behandlungspraxis (und insgesamt der Intergeschlechtlichkeit) auf die individuelle Identität zu untersuchen (vgl. Schweizer \& RichterAppelt, 2012b, S. 190f.).

Als ein wesentliches Resultat der Studie ist zu nennen, dass der Prozess der medizinischen Diagnostik von den Behandelten als außerordentlich belastend erlebt wird. Beanstandet werden neben dem zeitlichen Ausmaß ganz konkret die fehlende Sensibilität und Empathie vieler Mediziner_innen. Inters* sehen in vielen Fällen ihre persönlichen Grenzen verletzt. Viele Situationen der Diagnosevermittlung wurden sowohl bezüglich der gewählten Sprache der Mediziner_innen als auch hinsichtlich der professionellen Haltung als unangemessen erlebt (vgl. ebd., S. 194). Die Ärzt_innen nahmen sich wenig bis keine Zeit, um die Diagnose adäquat-explizit zu vermitteln. Stattdessen erfolgte die Information oft beiläufig und unvollständig. Laut Aussagen von Studienteilnehmer_innen wurden so häufig binnen weniger Minuten ganze Welt- und Selbstbilder zerstört.

Kritik erfuhren darüber hinaus auch nicht-operative medizinische Behandlungsverfahren, darunter zum Beispiel Fotografien des Genitals zu Studienzwecken und/oder wiederholte genitale Untersuchungen, selbst in Anwesenheit von Studierendengruppen. Dabei erfolgten Vorbesprechung und Durchführung medizinischer Eingriffe ausschließlich mit dem Ziel einer genitalen bzw. chirurgischen $\gg$ Korrektur $\ll{ }^{23}$. Fast alle Befragten (96\%) wurden einer Hormontherapie

23 Die im medizinischen Sprachgebrauch häufig verwendete Bezeichnung »Korrektur« verharmlost bewusst den Tatbestand einer geschlechtszuweisenden und »vereindeutigenden« Genitaloperation. Diese Operationen werden durch die Abschwächung des Begriffs gesellschaftlich akzeptiert und legitimiert. 
unterzogen; mehr als die Hälfte (64\%) wurde gonadektomiert ${ }^{24}$ und genital ope$\operatorname{riert}^{25}(55 \%)$ - die meisten bereits im frühen Kindesalter. Ein Viertel berichtete von operativen Folgeeingriffen (vgl. ebd.).

60 Prozent der Befragten berichteten von depressiven Symptomen und Ängsten, die aufgrund des Leidensdrucks bei der Mehrzahl als behandlungsbedürftig einzustufen seien (vgl. Schweizer \& Richter-Appelt, 2012b, S. 196f.). Auch das körperliche Wohlbefinden sei durch eine hohe Unsicherheit hinsichtlich des subjektiven Körpererlebens gekennzeichnet (vgl. ebd., S. 196f.). Ebenso zeigt sich, dass die Bereiche Sexualität und Partner_innenschaft von den Behandelten als problematisch erlebt werden und einen hohen Belastungsgrad aufzeigen. Die Autor_innen regen deshalb an, »sexuelle Gesundheit nicht nur unter dem Aspekt »heterosexuellen Funktionierens zu betrachten «, sondern eine ganzheitliche Sicht einzunehmen « (Schweizer \& Richter-Appelt, 2012b, S. 197). Als positiv unterstützende Maßnahmen heben sie Psychotherapien, psychologische Gespräche und die Teilnahme an Selbsthilfegruppen hervor (vgl. ebd.). Anhand der Mehrfachnennungen hoher psychischer Belastungsgrade schlussfolgert die Hamburger Forscher_innengruppe daraus drei mögliche Ursachen, die für die gefühlte Beeinträchtigung der Lebensqualität infrage kommen und in Form eines interdependenten Gefüges wirkmächtig sind: die Annahme eines äußerlich untypisch erscheinenden Geschlechts, »die psychische und physische Bewältigung medizinischer Interventionen sowie psychosoziale Faktoren wie das Fehlen oder Vorhandensein von Ressourcen und Unterstützung $\ll$ (ebd.).

Im Vergleich zu den anderen hier vorgestellten Studien ist für die Hamburger Studie besonders die Art und Weise der Betrachtung und Einbeziehung der Lebensumstände und -realitäten intergeschlechtlicher Menschen als positiv hervorzuheben. Zwar werden auch hier medizinische Diagnosen klar differenziert dargestellt, dennoch vermitteln die schriftlichen Ausführungen den Eindruck, dass es vorrangig um eine ganzheitliche Betrachtung des Menschen ${ }^{26}$ geht und keine medizinische Störung im Mittelpunkt der Untersuchung stehen soll. Psychosoziale Problemlagen werden kontextualisiert und realitätsnah interpretiert.

24 Gonadektomie bezeichnet die chirurgische Entfernung der Keimdrüsen.

25 Zu den genannten Operationen zählen in diesen Fällen vornehmlich chirurgische Eingriffe am äußeren Geschlechtsorgan oder an der Harnröhre, chirurgische Vaginaldehnungen sowie Vaginalplastiken (vgl. ebd.).

26 Sowohl Hertha Richter-Appelt als auch Katinka Schweizer verfügen über eine Zusatzausbildung als Psychotherapeut_in - der Ansatz einer ganzheitlicheren Sicht des Menschen könnte daraus resultieren. 
Die kritische Haltung der Autor_innen ${ }^{27}$ zum bestehenden Behandlungsmodell - und darüber hinaus - kommt deutlich zum Vorschein:

»Intersexuelle Erscheinungsformen stellen das herkömmliche gesellschaftlicheZweiGeschlechter-System infrage. Gleichzeitig hat gerade dieses Modell das medizinische Vorgehen bei Intersexualität jahrzehntelang geprägt. Die meisten Teilnehmenden wurden entsprechend des lange geltenden >Optimal Gender Paradigmas< behandelt, d. h. es wurde versucht, mit medizinischen Maßnahmen eine bestmögliche Geschlechtszuordnung vorzunehmen « (Schweizer \& Richter Appelt, 2012b, S. 198).

Darüber hinaus unterstützen die Autor_innen der Studie die kritische Anmerkung einer Teilnehmer_in, dass es doch darum gehe, »Intersexualität anzuerkennen, nicht auszulöschen « (ebd.). Es geht den Autor_innen damit nicht nur um eine wissenschaftliche Abhandlung, sondern sie geben auch wertvolle und klient_innenorientierte Empfehlungen und Alternativen für die (Behandlungs-)Praxis. Zum »gerechten « Umgang mit Intergeschlechtlichkeit wird erwähnt, dass »Betroffenen « verschiedene Umgangsweisen anzubieten seien, die aufrichtige Aufklärung, Offenheit und Respekt erfordern und einen Handlungsspielraum im Entscheidungsprozess ermöglichen (vgl. Schweizer \& Richter-Appelt, 2012b, S. 199). Es sollen aber nicht nur körpermedizinische Möglichkeiten aufgezeigt, sondern vor allem auch solche der psychosozialen Unterstützung für Inters* und ihre Angehörigen angeboten und vermittelt werden (vgl. ebd.) - eine wichtige Schnittstellenfunktion, die im Verlauf der vorliegenden Arbeit erneut aufgegriffen wird.

\section{Erhebung des Deutschen Ethikrates}

Die Studie im Auftrag der Bundesregierung ${ }^{28}$ wurde 2011 in Form einer OnlineUmfrage »Zur Situation intersexueller Menschen « begonnen. Erstmalig sollten Daten zu den Lebenslagen von Inters* explorativ erfasst werden. Ziel der Erhebung

27 Obwohl der wissenschaftliche Beitrag von Schweizer und Richter-Appelt hier als zentral geführt wird und sie darüber hinaus auch Kritik am bestehenden Behandlungsmodell üben, werden sie dennoch auch von Inter*-Verbänden verschiedentlich kritisiert.

28 Bereits 2009 hatte das »UN Committee on the Elimination of Discrimination against Women《 die Bundesregierung aufgefordert, »wirksame Maßnahmen zum Schutz ihrer Menschenrechte zu ergreifen« (Deutscher Ethikrat, 2012a, S. 9f.). Diese beauftragte daraufhin den Deutschen Ethikrat, eine Stellungnahme zur Lebenssituation und Behandlungszufrieden- 
war es, »den Dialog mit den von Intersexualität betroffenen Menschen und ihren Selbsthilfeorganisationen fortzuführen und ihre Situation und die damit verbundenen Herausforderungen umfassend und unter Einbeziehung der ärztlichen, therapeutischen, sozialwissenschaftlichen und juristischen Sichtweisen aufzuarbeiten $[\ldots] \ll$ (Deutscher Ethikrat, 2012a, S. 9). Die Verbreitung des Fragebogens wurde von den Inters*-Selbsthilfegruppen unterstützt. Dies trug im Wesentlichen zur aktiven Teilnahme und zum Erfolg der Studie bei (vgl. Bora, 2012, S. 7).

199 Teilnehmende im Alter von 0 bis 67 Jahren (vgl. ebd., S. 7f.) nahmen an der Studie teil. Sie wurden aufgefordert, zu ihren Erfahrungen »bezüglich medizinischer Behandlungen, Einschätzungen der eigenen Lebensqualität und Einstellungen zu Fragen der kulturellen und gesellschaftlichen Perspektive « (Deutscher Ethikrat, 2012a, S. 13) Stellung zu nehmen. Aufgrund der großen Altersspanne der Teilnehmenden wurde eine Einteilung in drei Gruppen vorgenommen: 0-9 Jahre, 10-17 Jahre und über 17 Jahre. Es ist davon auszugehen, dass der Großteil der Fragebogen der unter Neunjährigen entweder ausschließlich von oder gemeinsam mit den Eltern ausgefüllt wurde. Aufgrund dieser Annahme zählt die Studie für die Gruppe der jüngsten Befragten zwar als wichtiger Erkenntnisgewinn, entspricht aber nicht den eigentlichen Kriterien, die darauf zielten, Betroffene selbst zu befragen.

Den Ergebnissen zufolge haben 95 Personen der Gesamtgruppe einen invasiven Eingriff ${ }^{29}$ und/oder eine hormonelle Behandlung erfahren. Bei ca. 70 Prozent fand dieser Eingriff bereits im Vorschulalter statt, bei der Hälfte der Befragten bereits vor Vollendung des zweiten Lebensjahres (vgl. Lenz et al., 2012, S. 33; Bora, 2012, S. 15). Auch der Folgeeingriff fand bei einem Drittel der Befragten im Vorschulalter statt. ${ }^{30}$ Zwei Drittel dieser Personen gaben

heit intergeschlechtlicher Menschen in der Bundesrepublik zu verfassen. Ihre Veröffentlichung 2012 initiierte zwar eine politische Auseinandersetzung mit Intergeschlechtlichkeit, berücksichtigte aber aus der Sicht von »Betroffenen« und deren Angehörigen nur unzureichend die zentralen Forderungen der Inter*-Verbände (vgl. Voß, 2012a, S. 6). In der Folge verabschiedeten einzelne Bundesländer Aktionspläne zur rechtlichen Gleichstellung sexueller und geschlechtlicher Vielfalt, und auf Bundesebene wurde im November 2013 das Gesetz zur Personenstandsfrage neu konzipiert. Dennoch werden medizinische Interventionen zur Geschlechtszuweisung und -vereindeutigung weiterhin nicht (explizit) als menschenrechtswidrig angesehen (vgl. Tolmein, 2012).

29 Zu den invasiven chirurgischen Eingriffen zählen hormonell abschließende Eingriffe (z. B. Gonadektomie, Kastration, Eierstockentfernung) sowie plastische und funktionale Veränderungen der Geschlechtsorgane, darunter z. B. Penisamputation, Klitorisektomie, Neovagina (vgl. Bora, 2012, S. 14).

30 Zu beachten ist hierbei, dass diese Aussagen auch seitens der Eltern über ihre Kinder getroffen wurden bzw. in Zusammenarbeit mit den Kindern. 
an, in den Entscheidungsprozess einbezogen worden zu sein (vgl. Bora, 2012, S. 16). Unklar bleibt hierbei, ob das zumindest bei der Gruppe der Jüngsten auch die Eltern subsumiert, die stellvertretend für ihre Kinder entschieden. Ist dies der Fall, so werden Selbstentscheidungen und Elternentscheidungen gleichbehandelt. Eine exaktere Ergründung der zustimmenden Akteur_innen fehlt. Es ist zu kritisieren, dass die Studie diese Differenzierung unterlässt. Während ein Viertel aller Proband_innen von ihrer Intergeschlechtlichkeit bis zum elften Lebensjahr erfahren haben, geben immerhin noch 17 Prozent an, bereits über 30 Jahre alt gewesen zu sein, als sie davon in Kenntnis gesetzt wurden (vgl. Bora, 2012, S. 12). In Bezug auf die individuelle Lebensrealität werden vor allem Erfahrungen in den Bereichen Diskriminierung (ca. 27\%), Hemmschwellen und Krankenversicherung ${ }^{31}$ (ca. 9-12\%) als problematisch ${ }^{32}$ angesehen. So berichten die Teilnehmenden von häufigen Ausgrenzungserfahrungen. Sie benennen Schwierigkeiten mit der (Fremd-)Zuordnung innerhalb des Zwei-GeschlechterModells und klagen über »körperliche Gewalt, fehlende Aufklärung und [die] Verwechslung mit Transsexualität sowie falsche[] medizinische[] Behandlung und berufliche[] Nachteile[] (Bora, 2012, S. 19). Die Inter*-Selbsthilfe berichtet dazu:

$»$ Viele Betroffene sind aufgrund der durch die Behandlung verursachten psychischen und physischen Schäden erwerbsunfähig und fristen ein prekäres Dasein « (Truffer, 2011, S.3).

Die Behandelten leiden insbesondere unter der gesellschaftlichen Tabuisierung (ca. 16\%) von Intergeschlechtlichkeit. Darunter fallen vor allem das Verstecken in der Öffentlichkeit und die damit verknüpften Entscheidungsschwierigkeiten im Alltag, zum Beispiel beim Aufsuchen der Toilette. Ausgrenzung und Intoleranz als alltägliche Hürden führen in vielen Fällen auch zu Problemen im Berufsleben $^{33}$ (vgl. Bora, 2012, S. 20f.). Ein weiteres großes Problem stellt die konkrete Kommunikation mit den Krankenkassen dar. Erwähnt werden in erster Linie

31 Immer wieder berichten Inters* von Konflikten mit ihren Krankenkassen, wenn es um Abrechnungs- und Bewilligungsfragen bei der spezifischen intergeschlechtlichen Diagnose geht. Häufig werden vor allem Schwierigkeiten mit der geschlechtsspezifischen Einordnung und der Verwechslung mit Transsexualität/Transgender benannt.

32 Bei den folgenden Punkten benenne ich bewusst die auffallend negativen Ergebnisse, um noch bestehenden Handlungsbedarf sichtbar zu machen.

33 Auch hier wird eine intersektionale Verschränkung struktureller Probleme deutlich. So kommt es bei der Suche nach einem Ausbildungs- oder Arbeitsplatz nicht aufgrund der 
Abrechnungs- und Bewilligungsschwierigkeiten und die Forderung hoher Prämien bei Privat- und Zusatzversicherungen. Besondere Schwierigkeiten bestehen in Bezug auf die geschlechtsspezifische Zuweisung. Beispielhaft geben Inters* an, es sei »ein unangenehmes Gefühl, mit einer personenspezifischen Anrede (Frau/Herr) bei optischer Uneindeutigkeit oder bei optisch gegensätzlichem Erscheinungsbild in der Arztpraxis aufgerufen zu werden « (ebd.).

Generell bestätigt die Studie die Annahme, dass chirurgische Maßnahmen in unterschiedlichen Bereichen Auswirkungen auf die Lebensqualität haben. Im Speziellen werden negative Effekte auf die Geschlechterrolle und die psychische und körperliche Gesundheit benannt. So sprechen 40 Prozent von einer niedrigen Lebenszufriedenheit (vgl. Bora, 2012, S. 22). In Bezug auf die sexuelle Lebensqualität werden, wie in den anderen beiden Studien auch, große Unzufriedenheiten bei den (medizinisch behandelten) Inters* erhoben. Aussagen darüber reichen von einem als negativ empfundenen Aussehen der Geschlechtsorgane über ihre Funktion und die damit verbundene sexuelle Inaktivität bis hin zu belastenden sexuellen Problemen, Ängsten und Unsicherheiten in zwischenmenschlichen und sexuellen Situationen (vgl. Wunder, 2012, S. 38).

Bestrebungen und Wünsche der Studienteilnehmenden werden in vielen Bereichen deutlich. Unter dem Aspekt der Aufklärung werden insbesondere Bildungsinstitutionen angesprochen und Multiplikator_innen-Schulungen auch für medizinisch-pflegerisches Fachpersonal eingefordert. Von grundsätzlicher Priorität sind dabei die generelle Enttabuisierung von Intergeschlechtlichkeit (30\%) und die Durchsetzung eines prinzipiellen Operationsverbotes im Kindesalter (ca. 16\%), mit Ausnahme des medizinischen Notfalls. Darüber hinaus verlangen 20 Prozent der befragten Inters*, dass unterstützende Einrichtungen und Hilfsangebote sowie rechtlich anerkannte Entschädigungsleistungen (ca. 7\%) für die an ihnen verübten Eingriffe etabliert werden. Forderungen nach Überwindung des Zwei-Geschlechter-Systems (ca. 16\%) gehen mit solchen nach Veränderungen im Personenstandsrecht ${ }^{34}$ (ca. 18\%) einher (vgl. Bora, 2012, S. 30f.).

Intergeschlechtlichkeit zu Schwierigkeiten, sondern aufgrund der äußeren und gesellschaftlichen Strukturen bzw. der strukturellen Diskriminierung, denen Inters* ausgesetzt sind (Mobbing). Dennoch ist die Schilderung dieser Situation ein geeignetes Beispiel für die Inter*-Beratungspraxis.

34 Seit dem 1. November 2013 gilt bundesweit, dass der Personenstand eines Kindes, sofern es weder eindeutig dem männlichen noch dem weiblichen Geschlecht zugeordnet werden kann, ohne eine solche Angabe in das Geburtenregister einzutragen sei. Diese Neuregelung im Personenstandsgesetz geht auf die Initiative des Deutschen Ethikrates zurück (vgl. Voß, 2013). 
Abschließend überwiegen Einstellungen, Operationen im Kindesalter abzulehnen, das Erziehungsgeschlecht offen zu lassen und geschlechtszuweisende und -vereindeutigende Operationen nur im einwilligungsfähigen Alter durchzuführen. Diese sollten ausnahmslos an die » persönliche informierte Einwilligung der betroffenen Person « (ebd., S. 27f.) gebunden sein. Diese Einstellungen spiegeln zwar in gleichem Maße öffentliche wie auch politische Forderungen der Inters*Selbstorganisationen wider, werden jedoch nicht ausdrücklich seitens des Ethikrates postuliert. Dieser fordert »lediglich « Folgendes (vgl. Deutscher Ethikrat, 2012b):

$>$ die gesellschaftliche Integration und Enttabuisierung von Inters* in Deutschland (Anerkennung, Unterstützung, Schutz vor gesellschaftlicher Diskriminierung)

$>\quad$ einen Aufschub medizinischer Eingriffe bis zur Entscheidungsfähigkeit des Kindes

$>$ die Durchführung von Diagnostik und Behandlung ausschließlich durch spezialisierte interdisziplinäre Kompetenzteams, bestehend aus Ärzt_innen und Expert_innen anderer Disziplinen

$>$ die Gewährleistung weiterführender Betreuung in unabhängigen Beratungs- und Betreuungseinrichtungen (einschließlich Peer-Beratung)

$>\quad$ eine Änderung des Personenstandsrechts mit der Option »anderes « neben der Zuordnung » männlich « oder » weiblich «

Wesentliche Kritikpunkte an den Schlussfolgerungen des Ethikrates sind zum einen die Nicht-Positionierung zur Forderung nach genereller Abschaffung der geschlechtsvereindeutigenden und -zuweisenden Eingriffe im Kindesalter, zum anderen die Fokussierung auf die ICD-1035/DSM IV36-Diagnoseschlüssel, die als streng voneinander getrennt aufgefasst werden. Daraus folgt eine Teilung in vor allem zwei Gruppierungen: Die als Adrenogenitales Syndrom ${ }^{37}$ (AGS) erfassten

35 ICD-10: die Internationale statistische Klassifikation der Krankheiten und verwandter Gesundheitsprobleme

36 DSM-IV: Diagnostischer und Statistischer Leitfaden zu psychischen Störungen

37 Das Adrenogenitale Syndrom ist auch unter der Bezeichnung Nebenniereninsuffizenz bekannt. Aufgrund eines erblich bedingten Enzymmangels kann der Körper nicht ausreichend Kortisol produzieren. Es kommt daher zu einer Vergrößerung der Nebenniere, was einerseits die Kortisolbildung unterstützt, andererseits aber auch zu einer Überproduktion von Androgenen führt. Zudem kann ein Aldosteronmangel vorliegen, der nach der Geburt zu einer lebensbedrohlichen Stoffwechselkrise führen kann. Dieser Mangel ist zwar medikamentös (mit Kortison, Aldosteron) behandelbar, muss aber lebensbegleitend erfolgen (vgl. Groneberg \& Zehnder, 2008, S. 225). 
und explizit benannten Diagnosen werden allen anderen Formen intergeschlechtlicher Varianz ${ }^{38}$ gegenübergestellt.

\section{Ansätze einer kritischen Forschung}

Ein Vergleich der dargestellten Studienergebnisse führt zu folgender zusammenfassender Erkenntnis: Trotz unterschiedlicher Herangehensweisen kommen alle drei Studien zu dem Resultat, dass medizinische Interventionen einen erheblichen Einfluss auf die Lebenslagen intergeschlechtlicher Personen haben. Das Ausmaß erstreckt sich über verschiedene Altersstufen und Phasen der Entwicklung, betrifft darüber hinaus aber auch sehr persönliche Lebens- und Erfahrungsbereiche, sowohl in psychischer als auch in physischer Hinsicht. Die Ergebnisse zeigen, dass Veränderungen des medizinischen Handelns und Beratens erforderlich sind.

Ein ressourcenorientiertes Verständnis von Intergeschlechtlichkeit, abseits pathologisierender Zuschreibungen, bedarf allerdings auch bestimmter Auseinandersetzungen mit der eigenen Rolle und den eigenen Sichtweisen als wissenschaftlich und forschend Tätige_r. Die Hamburger Forschungsgruppe bestätigt, wie sehr ein Sich-Einlassen auf andere Lebenswelten die eigenen Sichtweisen verändern und positiv beeinflussen kann:

»Aus den Gesprächen und Begegnungen mit den teilnehmenden Menschen haben wir weit mehr gelernt als statistisch oder inhaltsanalytisch ausgewertet und dargestellt werden kann. Eine wesentliche Erfahrung war, dass sich unsere eigenen Vorstellungen und Haltungen im Verlauf des Projektes fortlaufend weiterentwickelt und dadurch auch verändert haben « (Schweizer \& Richter-Appelt, 2012b, S. 192).

38 Der Deutsche Ethikrat begründet diese terminologische Differenzierung damit, dass ein Großteil der AGS-»Betroffenen« in Kommentaren darauf verwiesen habe, dass Menschen mit AGS nicht zur Gruppe der Inters* gezählt werden sollen, da sie sich nicht als inter* fühlen (vgl. Bora, 2012, S. 10). Dies könnte unter Umständen daran liegen, dass ihre Form der Intergeschlechtlichkeit aus gesundheitlichen Gründen behandelt werden müsse und ihnen keine Entscheidungsmöglichkeit bei medikamentöser Behandlung obliege (vgl. ebd., S.16). Bezugnehmend auf diese Studie unterscheiden sich die Antworten beider Gruppen in vielen Fällen signifikant. Eine Begründung für die m. E. mehrheitsgesellschaftliche Argumentation der Personengruppe mit AGS kann auf die fehlende Identifikation mit der Intergeschlechtlichkeit zurückgeführt werden. In dieser Arbeit werden deshalb die Aussagen derer verstärkt in den Blick genommen, die sich unter der Bezeichnung Inter* wiederfinden. 
In ihren Schlussfolgerungen zur Studie regen die Hamburger Expert_innen an, dass die Medizin unter dem Eindruck der Expertise von Theorien zu nicht-diskriminierender Geschlechtsidentität ihr Verständnis von der und ihre Haltung zur psychosexuellen Entwicklung bei Intergeschlechtlichkeit verändern und so zu einer Neukonzeption beitragen solle (vgl. ebd., S. 200). Dabei stellen die Autor_innen wichtige Überlegungen dazu an, wie sich für sie als Wissenschaftler_innen gegenüber der eigenen, aber auch jeder einzelnen Teildisziplin (z.B. Medizin, Psychologie, Pädagogik, Recht) eine kritische Grundhaltung bezüglich Inter* entwickeln lasse. Betont werden insbesondere die Themen Identitätsbildung und Stärkung der Selbstsicherheit. Fachlich empfehlen die Expert_innen den beratenden Mediziner_innen, ihre persönliche Ambiguitätstoleranz zu erweitern sowie spezifische Kompetenzen im Bereich Krisenbewältigung bzw. psychologische Beratung in Bezug auf Inters* verschiedener Altersgruppen und ihre Angehörigen zu erwerben (vgl. ebd.). Zwei Grundprinzipien werden als Antwort auf die vielschichtigen Herausforderungen formuliert:

»time \& talk, Zeit und Zuhören - Zeit, um vermeintlichen Handlungsdruck überdenken und Entscheidungen rational und emotional prüfen zu können, und Zuhören im vertrauensvollen Gespräch, das Denk- und Reflexionsräume öffnet « (ebd.).

In diesem Zusammenhang empfehlen die Autor_innen weitere relevante Themen für eine wissenschaftliche Auseinandersetzung: eine Vergleichsstudie zur Lebensqualität von Inters*, die geringe medizinische Behandlungserfahrungen haben, das Aufklärungserleben von Klient_innen und eine Erhebung zu den Erfahrungen von Eltern. Unter dem Aspekt einer kritischen Selbstreflexion schlagen Schweizer und Richter-Appelt vor, individuelle Behandlungsmöglichkeiten zu entwickeln, um die impliziten Indikationsmodelle der Behandelnden selbst zu überdenken (vgl. ebd., S. 201). Zu diesen und weiteren Forschungsvorhaben verweisen sie auf Michael Groneberg und Katrin Zehnder (Groneberg \& Zehnder, 2008, S. 221), die einen intersubjektiven Forschungsprozess vorschlagen (vgl. Schweizer \& Richter-Appelt, 2012b, S. 201) und damit zu einer kritischen, verantwortungsvollen Forschung beitragen wollen. Diesbezüglich ist ergänzend anzumerken, dass auch das Abgreifen der Expertise von Inters* zu Forschungszwecken kritisch betrachtet bzw. in angemessener Form anerkannt und entlohnt werden muss.

Dem genannten Reformierungsgedanken schließt sich Ulrike Klöppel mit ihrer wissenschaftlichen Kritik an der Studie des Ethikrates an. Klöppel kritisiert 
die starre, direkt zu Beginn der Studie positionierte Abfrage von Diagnosen und schlussfolgert, dass dieses Vorgehen viele Inters* von einer Studienteilnahme abhalte bzw. abgehalten habe (vgl. Klöppel, 2012a, S. 93). Genauso seien diejenigen Stimmen unter der Gruppe der AGS-»Betroffenen « ignoriert worden, die sich ebenfalls kritisch zu den medizinischen Eingriffen äußerten (vgl. ebd.). Klöppel führt dies auf ein wenig reflektiertes und biologistisches Geschlechterverständnis seitens des Ethikrates zurück, der eine höhere Lebenszufriedenheit per se mit einer eindeutigen Geschlechtlichkeit verbinde. Damit würden willkürliche Zuschreibungen, Homogenisierungen und Ausschlüsse durch die diagnostische Einteilung reproduziert (vgl. ebd.). Klöppel wirft in diesem Zusammenhang die Frage auf, warum medizinischer Expertise mehr Gewicht zuteil werde als den Urteilen und Aussagen von Patient_innen (vgl. Klöppel, 2012b, S. 28). Aus der Kritik dieser Beobachtung heraus postuliert Klöppel, dass auch die wissenschaftliche Forschung einen herrschaftskritischen Standpunkt einnehmen und eigene Erfahrungshintergründe stets mitdenken und reflektieren müsse (vgl. Klöppel, 2012a, S. 92ff.).

\section{Zur Bedeutung des Peer-Ansatzes: Selbsthilfe und Organisationen}

Seit Beginn der 1990er Jahre formiert sich Widerstand »Betroffener « gegen den tabuisierenden und diskriminierenden Umgang mit Intergeschlechtlichkeit (vgl. Voß, 2012a, S. 15). Selbsthilfeorganisationen haben sich seitdem zu einem wissenschaftlich ernst zu nehmendem Grundpfeiler im Umgang mit Intergeschlechtlichkeit institutionalisiert. Die erste Generation derer, die dem Baltimorer Behandlungsprogramm unterzogen wurden, war nun in einem Alter, die wahren Gründe der vielen Krankenhausaufenthalte ihrer Kindheit in Erfahrung bringen zu können (vgl. ebd.) und damit das gesellschaftliche Schweigegebot zu brechen. Die neugewonnenen Erkenntnisse werden oft als schmerzvoll erlebt:

»Ich versuche, so offen wie nur möglich mit diesem Thema umzugehen, aber es schmerzt immer wieder, mich mit meiner ganz persönlichen Vergangenheit auseinanderzusetzen. Denn ich glaubte immer ganz fest daran, dass alles nur zu meinem Besten war. Es wurde mir nichts gezielt verheimlicht. Dennoch brach eine Welt zusammen, als die Wahrheit zum Vorschein kam. Zurück bleibt ein Trümmerfeld, welches unüberschaubar ist und welches ich früher oder später aufräumen muss « (Eveline, 2008, S. 24). 
Nach einem ersten Einblick in die Krankenakten fühlten sich die meisten mit ihrer Diagnose zunächst alleingelassen. Die mit dem Internet aufgekommenen neuen Kommunikationsformen ermöglichten bzw. vereinfachten einen selbstständigen Austausch zwischen Inters* (vgl. Voß, 2012a, S. 16). Diese neuartige Vernetzung resultierte in der Gründung verschiedener Zusammenschlüsse, die einen immensen Effekt auf kollektive Identitätsbildungs- und Organisierungsprozesse von intergeschlechtlichen Menschen hatte. Das Jahr 1993 gilt dabei als Initialisierungszeitpunkt der Inter*-Verbände ${ }^{39}$. Erstmalig formulierten sie nun öffentlich Kritik am bisherigen chirurgisch-kosmetischen Behandlungsmodell (vgl. Klöppel, 2012a, S. 92). Obwohl sich in den letzten 20 Jahren innerhalb der wissenschaftlichen Forschung progressiv viel bewegt hat, werden die Forderungen der Aktivist_innen nach mehr Sensibilität und einer kritischen und respektvollen Forschung immer noch nicht ausreichend berücksichtigt (vgl. ebd.).

Karin Plattner, selbst Mutter eines intergeschlechtlichen Kindes und Vorsitzende_r des Vereins Selbsthilfe Intersexualität (SI), berichtet von dem schmerzhaften Gefühl, nach der Geburt von den Ärzt_innen mit ihrer_seiner Situation völlig alleingelassen worden zu sein (vgl. Plattner, 2008, S. 13).

»Allgemeine Ratlosigkeit machte sich breit, niemand schien die Situation im Griff zu haben und keiner wusste recht, wie er sich uns gegenüber verhalten sollte. [...] Rückblickend kann ich sagen, dass wir als Eltern sowohl mit der Unsicherheit der Ärzte, als auch durch das fehlende Fachwissen total überfordert waren « (Plattner, 2008, S. 14).

Nach intensiver Recherche im Internet fanden die Eltern Anschluss an eine $\gg$ Betroffenengruppe « und gründeten daraufhin 2003 eine Selbsthilfegruppe (vgl. ebd). Der Verein SI setzt sich für eine Enttabuisierung von Inter* ein und wendet sich gegen geschlechtszuweisende und geschlechtsvereindeutigende Operationen im Kindesalter (vgl. Groneberg \& Zehnder, 2008, S. 9). Zudem unterstützt er eine bessere Informationspolitik (vgl. Plattner, 2008, S. 13). Als wesentliches Vereinsziel wird formuliert, einen Erfahrungsaustausch »betroffener « Eltern, von Inters* und Unterstützer_innenkreisen zu ermöglichen und zu fördern. Plattner sieht in dem Verein SI die Möglichkeit, sich international zu vernetzen, Gehör bei

39 Cheryl Chase gilt als Schlüsselfigur und Pionier_in der Inter*-Bewegung in den USA. Inspiriert von den Aufsätzen zu Geschlecht der Autor_innen Anne Fausto-Sterling und Suzanne J. Kessler gründete Chase 1993 die Intersex Society of North America (ISNA), die bis heute eine der bedeutendsten Vereinigungen auf internationaler Ebene darstellt (vgl. Voß, 2012a, S. 16f.; Intersex Society of North America, o. J.). 
bedeutsamen Körperschaften und Organisationen von Ärzt_innen und Psycholog_innen zu finden und einen Zugang zu Presse und Öffentlichkeit zu erlangen, um auch politische Forderungen in Bezug auf rechtliche Regelungen (z. B. Personenstandsgesetz) zu stellen (vgl. ebd., S. 17). Die Selbsthilfe Intersexualität plädiert dafür, in die Aus- und Weiterbildung von Hebammen, Lehrpersonal und Psycholog_innen zu investieren, um Inters* und ihr Umfeld bei der Problembewältigung zu unterstützen (vgl. ebd.).

Die diskursive Auseinandersetzung um Intergeschlechtlichkeit ist mittlerweile durch die aktive Beteiligung »betroffener « Einzelpersonen und medizinkritischer Selbsthilfegruppen geprägt (vgl. Zehnder, 2008, S. 26). Wie Zehnder in ihrer_seiner Untersuchung von Selbstzeugnissen intergeschlechtlicher Menschen herausgefunden hat, kommt eben nicht der Familie, sondern vor allem der Selbsthilfe eine tragende Rolle bei der Bewältigung persönlicher Problemlagen im Zusammenhang mit der eigenen Intergeschlechtlichkeit zu (vgl. ebd., S. 27). Selbsthilfe wird dort als wichtigste Ressource benannt und somit, schlussfolgert Zehnder, das Thema Inter* im Kreis der Peers erstmalig positiv besetzt (vgl. ebd.).

Der inzwischen auch in der deutschsprachigen Forschung verwendete englische Begriff »Peer « bedeutet: Gleichstehende_r, Ebenbürtige_r oder Fachkolleg_in. Im Bereich des »Peer-Counseling « wird Peer als Beratung auf Augenhöhe verstanden (vgl. Morgen, 2013, S. 122). »Betroffene « (Peers) beraten andere »Betroffene « (Peers) und greifen auf eine gemeinsam geteilte Erfahrung zurück (vgl. ebd.). Peer-Berater_innen gelten somit als Expert_innen aufgrund ihrer eigenen intergeschlechtlichen Biografie (Psychenet, 2013). Diese Art von Beratung wird insbesondere von den Selbsthilfegruppen angestrebt und postuliert (vgl. ebd., S. 122).

Neben dem persönlichen Austausch innerhalb der Selbsthilfegruppe ist es insbesondere die Wiedergabe persönlicher Erfahrungsberichte und Geschichten - vor allem in Form von Büchern, Artikeln und auf Webseiten -, die eine groBe Bedeutung für die eigene Identifikation und Vernetzung hat. So berichtet die Gruppe der XY-Frauen ${ }^{40}$, dass es hilfreich sein kann, »seine persönlichen Erfahrungen mitzuteilen, sowohl für sich selbst als auch für andere « (Zehnder, 2008, S. 31). Zehnder erläutert in ihrer Analyse weiter, dass der Erstkontakt zu einer Selbsthilfegruppe von Inters* meist als Wendepunkt im Leben empfunden wird. Für die meisten Inters* ist es durch den Kontakt zu einer Selbsthilfegruppe das

40 Die Gruppe der XY-Frauen ist ein Selbsthilfeverband des Vereins Intersexuelle Menschen. (vgl. XY-Frauen, O. J.). 
erste Mal überhaupt möglich, dass sie andere »Betroffene « kennenlernen, offen über ihre Varianz sprechen können und sich verstanden fühlen. Diese Gruppe nimmt folglich eine wesentliche Rolle im Leben ein und eröffnet nicht zuletzt einen neuen positiven Lebensabschnitt (vgl. ebd., S. 35). Ein Mitglied der Selbsthilfegruppe XY-Frauen beschreibt dieses Zugehörigkeitsgefühl folgendermaßen:

»Die Selbsthilfegruppe ist für mich wie ein Stück Familie geworden. In der Begegnung mit anderen AIS ${ }^{41}$ Frauen geschieht sehr viel Heilung. Über das schreckliche Geheimnis, das man so lange gehütet hat, kann man endlich in aller Offenheit reden $\ll$ (Anna, 2001).

Im nächsten Abschnitt werden nun drei der wichtigsten Selbstorganisationen im deutschsprachigen Raum vorgestellt und ihre Positionen und Haltungen hinsichtlich inter*-spezifischer Forderungen dargelegt. Alle drei eint, dass sie Selbsthilfe als fundamentale Unterstützungs- und Selbstermächtigungsmöglichkeit ansehen. Ihre übergeordneten Ziele sind einerseits die Thematisierung und Sichtbarmachung von Inter* im öffentlichen Raum, andererseits die Absage an eine zweigeschlechtlich geprägte Gesellschaft. Die Kritik am medizinischen und rechtlichen Umgang sowie der damit einhergehenden Pathologisierung stellt darüber hinaus einen wichtigen Bestandteil ihrer täglichen Arbeit dar (vgl. Wunder, 2012, S. 34). Das Schlaglicht auf diese drei Vereinigungen dient dazu, Entstehung, Hintergründe und Nutzen von Selbsthilfe nachzuzeichnen, insbesondere unter dem Aspekt der individuellen Bedeutsamkeit, sowohl für die Beratung als auch für das Selbstwertgefühl von Inters*.

\section{Bundesverband Intersexuelle Menschen e.V.}

Hervorgegangen aus der Selbsthilfegruppe der XY-Frauen, gründete sich am 17. April 2004 der Verein Intersexuelle Menschen e.V. (IMeV), der »gemeinsame Ziele und Forderungen von Intersexuellen aller Altersgruppen im deutschsprachigen Raum vertreten und auch die Interessen von Angehörigen intersexueller Menschen wahrnehmen soll « $(\mathrm{IMeV}, 2011$, S. 1). Unter dem Leitsatz »Aus

41 AIS ist eine Kurzform der Bezeichnung Androgenresistenz respektive Androgeninsensitivitätssyndrom, auch als testikuläre Feminisierung bekannt. Sie ist genetisch bedingt und verzeichnet eine geringe oder ausbleibende Wirkung von Androgenen, die zu einer »Verweiblichung bei männlichen Gonaden« (Groneberg \& Zehnder, 2008, S. 226) führt. 
der Isolation in die gemeinsame Offensive « (ebd.) schlossen sich Menschen zusammen, die erlebt haben, wie sich ein tabuisierender Umgang mit ihrer Intergeschlechtlichkeit auf die Entwicklung der eigenen Identität und des Selbstbildes ausgewirkt hat (vgl. ebd.).

Der Verein mit Sitz in Hamburg »setzt sich ein für ein selbstbestimmtes, diskriminierungsfreies Leben aller Menschen. Intersexuelle Menschen e. V. steht ein für die Verwirklichung der Menschenrechte und wendet sich gegen jede Art der Diskriminierung und Benachteiligung wegen des Geschlechtes auf nationaler und internationaler Ebene « (ebd., S. 2).

Sieben Arbeitsschwerpunkte werden als Basisziele des Bundesverbandes formuliert (vgl. ebd.):

$>\quad$ die Unterstützung, Förderung und Ausbildung von Selbsthilfegruppen

$>\quad$ eine individuelle Beratung, Unterstützung und Hilfe zur konkreten Lebenssituation intergeschlechtlicher Menschen und ihrer Familien

$>\quad$ die Förderung und Unterstützung der Selbsthilfe von Eltern mit intergeschlechtlichen Kindern

$>$ Kooperation und Vernetzung mit weiteren Initiativen und Verbänden gleicher oder ähnlicher Zielsetzung

$>\quad$ eine kritische Beratung, Fort- und Weiterbildung politischer, gesellschaftlicher und medizinischer Einrichtungen

$>\quad$ der Aufbau eines Netzes landesspezifischer Selbsthilfe- und Beratungsstellen innerhalb der Bundesrepublik Deutschland

$>$ die Durchsetzung der individuellen Interessen der Organisierten im Verein

Sein Selbstverständnis von Öffentlichkeitsarbeit formuliert der Verein wie folgt:

»Wir sind daher nicht nur ein Sprachrohr für die Interessen von intersexuellen Menschen, sondern wirken durch Veranstaltungen, Ausstellungen, Publikationen und Auftritte in den Medien auch kulturbildend und beeinflussen aktiv den gesellschaftlichen Diskurs. Auch hier ist noch sehr viel zu tun. Wir mischen uns ein!« (ebd.).

Der Bundesverband ist in acht Landesverbände gegliedert und unterhält als bundesweites Netzwerk auch Kontakte in die Schweiz und nach Österreich. Aktive Mitgliedschaften sind exklusiv intergeschlechtlichen Menschen vorbehalten. Eltern, Angehörige und weitere Unterstützer_innen können als passive Mitglieder partizipieren und sind ausdrücklich eingeladen, »die Vereinsziele zu unterstützen, Lobbyarbeit zu leisten und im Sinne des Vereins gesellschaftlich 
und/oder politisch aktiv zu werden « (ebd., S. 4). Darüber hinaus stehen Fördermitgliedschaften prinzipiell allen Interessierten offen. Ein wissenschaftlicher Beirat unterstützt und berät den Verein (vgl. ebd.).

Erfolgreich vertrat der Verein die Rechte intergeschlechtlicher Menschen vor nachfolgenden UN-Ausschüssen (vgl. Barth et al., 2013, S. 121). In einem eigenen Schattenbericht zum Übereinkommen des UN-Ausschusses » zur Beseitigung jeder Form der Diskriminierung von Frauen (CEDAW) « aus dem Jahr 2008 kommentieren Vertreter_innen des Vereins Intersexuelle Menschen e.V. und der XY-Frauen ihre besondere Situation und betonten, dass sie »sehr spezifischen Formen von Gewalt und Diskriminierungen ausgesetzt sind « (IMeV \& XY-Frauen, 2008, S. 6). Menschenrechtsverletzungen an Inters* werden insbesondere für die Bereiche Gleichberechtigung, Nicht-Diskriminierung, Bildung, Gesundheit, Ehe und Familie aufgezeigt. Des Weiteren wird die Bundesregierung aufgefordert, zu den im Bericht formulierten Fragen Stellung zu beziehen (vgl. ebd., S. 7f.).

In einem Parallelbericht zur Anti-Folter-Konvention (CAT) aus dem Jahr 2011 fordern der IMeV und die XY-Frauen Deutschland auf, Stellung zu medizinischen Eingriffen zu beziehen. Sie führen aus: Intergeschlechtliche Kinder werden auch hierzulande » routinemäßig medizinischen Behandlungen unterworfen, die als Folter oder unmenschliche oder erniedrigende Behandlung einzustufen sind. Auch Jugendliche oder Erwachsene, bei denen später im Leben IntersexVariationen festgestellt werden, können solchen Behandlungen ausgesetzt sein « (IMeV \& XY-Frauen, 2011, S. 5). Als Beispiel für die Gewalt benennen sie die Entnahme reproduktiver Organe, die zur Sterilität führt und eine lebenslange Hormonsubstitution notwendig macht. Ein zweites Beispiel zeigt auf, dass bei einer operativen Feminisierung des Körpers eine Beschneidung der Klitoris erfolgt, die zum Verlust der sexuellen Empfindsamkeit führt. Nach Anlegung einer Neo-Vagina muss diese regelmäßig und unter Schmerzen bougiert (gedehnt) werden, um die sogenannte Penetrationsfähigkeit zu erhöhen (vgl. ebd.). Dass diese Behandlungsmethoden schwere psychische und physische Leiden verursachen, zeigen die Aktivist_innen anhand konkreter Fallbeispiele.

Auch Ergebnisse der vorliegenden Outcome-Studien belegen die Einschränkungen und Folgeerscheinungen operativer Eingriffe an intergeschlechtlichen Menschen (vgl. ebd., S. 6). Demzufolge konstatiert der Bundesverband:

»Menschen mit intersexueller Geschlechtsentwicklung sind ein Teil unserer Gesellschaft und haben als gleichberechtigte Bürger ${ }^{*} I n n e n$ ein Recht auf freie Entfaltung und Entwicklung. Die an ihnen begangenen medizinisch nicht notwendigen, trau- 
matisierenden Behandlungen stellen erhebliche Verstöße gegen die Menschenrechte dar. Sie verletzen das Recht der intersexuellen Menschen auf Selbstbestimmung und die Würde. Wir haben es uns deshalb zur Aufgabe gemacht, intersexuelle Menschen zu schützen sowie dafür einzutreten, dass keine weiteren Opfer dieser menschenrechtswidrigen Praxis mehr entstehen. Bestehende Opfer sind soweit als möglich zu entschädigen und zu rehabilitieren « (ebd.).

Aus dieser Grundhaltung heraus und orientiert an den Empfehlungen des Parallelberichts der CAT entwickelt der Verein fünf dezidierte Forderungen an Medizin, Gesellschaft und Politik:

$>$ Nicht lebens- oder gesundheitsnotwendige Eingriffe ohne die informierte Einwilligung der »betroffenen « Menschen sind generell zu unterlassen. Dazu zählen chirurgische, medikamentöse und hormonelle Methoden, die angewandt werden, solange keine medizinische Indikation vorliegt. Alle Eingriffe, inklusive kosmetischer Behandlungen, sowie alle möglichen Risiken im Zusammenhang mit diesen Eingriffen, müssen Inters* umfassend und vollständig in schriftlicher Form und mündlich erklärt werden. Dies gilt ebenso für die Aushändigung einer Kopie der Patient_innenakte nach der Entlassung aus der medizinischen Behandlung (vgl. IMeV, o.J.a).

$>$ Es sind verbindliche »Standards of Care « unter Einbeziehung intergeschlechtlicher Menschen und ihrer Organisationen zu schaffen. Zu diesen Standards gehören laut Verein eine flächendeckende Errichtung von Beratungsstellen, die Einrichtung spezifischer Kompetenzzentren sowie die spezialisierte Ausbildung von Fachpersonal und die Bildung von interdisziplinären Beratungsteams für Eltern und »Betroffene «. Zudem beinhaltet dieser Punkt die Absicherung von Fördermitteln und die Finanzierung dieser Angebote, darüber hinaus aber auch die Etablierung von Inters* als Beobachter_innen von Studien und Evaluierungen zum Thema (vgl. ebd.).

$>$ Das Thema Intergeschlechtlichkeit soll als verpflichtender Bestandteil in die Lehrpläne von Schulen und in Berufsausbildungen aufgenommen werden (vgl. ebd.).

$>$ Geschädigte »Betroffene « sind in Form eines Hilfs- und Entschädigungsfonds ausdrücklich zu entschädigen und zu rehabilitieren. Dieser soll finanziell sowohl vom Staat als auch von den Standesorganisationen der Medizin unterhalten werden. Beide Institutionen seien in der Verantwortung für die Fehlbehandlungen an Inters*. Ebenso sollen rentenrechtliche Regelungen getroffen, die Befreiung bei Krankenversicherungsbeitragszuschlägen erwirkt und Rehabilitationspläne entwickelt werden, um die gesundheit- 
liche Situation von Inters* wiederherzustellen und/oder zu stabilisieren. Dazu gehört die Anerkennung der infolge der Operationen entstandenen psychischen und körperlichen Beschwerden hinsichtlich eines Grades der Behinderung (vgl. ebd.).

$>$ Der Begriff Intersexualität soll Eingang in geltendes Recht finden. Das betrifft sowohl die im Jahr 2013 erwirkte Änderung im Personenstandsgesetz ${ }^{42}$ (vgl. Deutscher Bundestag, 2013, S. 3) als auch Forderungen nach Streichung der Verjährungsfristen im Falle nicht eingewilligter Behandlungen und das Verbot von Inter* als medizinische Indikation bei Schwangerschaftsabbrüchen (vgl. ebd.).

Der IMeV verfügt über mehrere Selbsthilfegruppen (SHG), die sich grundsätzlich an zwei Zielgruppen richten, nämlich zum einen an intergeschlechtliche Menschen allgemein (IMeV, o.J.a), zum anderen an XY-Frauen (IMeV, o.J.b). Zusätzlich verfügen beide über jeweils gesonderte Angebote für die Eltern-Selbsthilfe. In der Selbstbeschreibung der SHG XY-Frauen heißt es, diese sei eine »Kontaktgruppe für Menschen, die sich mit der Problematik auseinandersetzen müssen, nicht ohne weiteres in die gängigen Mann/Frau-Schemata hineinzupassen « (SHG XY-Frauen, o.J.). Weiterhin wird betont:

»In unserer Gruppe tauschen wir unsere eigenen Erfahrungen aus und setzen uns gemeinsam dafür ein, andere aufzuklären, Informationen und Gespräche anzubieten und den öffentlichen Dialog über diese biologische Spezialität anzuregen, zu steuern und idealerweise den empfindlichen Umgang mit diesem Thema zu entkrampfen $\ll$ (ebd.).

Die Elterngruppe berichtet, durch gegenseitigen Austausch Unterstützung zu erhalten, die »uns das Gefühl [gibt], nicht alleine zu sein. Dies hilft uns, unser Kind auf seinem Weg zu begleiten. Wir treffen uns, damit unsere Kinder einander kennenlernen können « (SHG Eltern, o.J.). Sowohl den Eltern als auch den Teilnehmer_innen der »Betroffenengruppe « ist die Entwicklung eines Gemein-

42 Eine Gesetzesänderung im Personenstandsrecht ist zwar erfolgt, stößt jedoch auf erhebliche Kritik der Inter*-Organisationen. Die bundesdeutsche Vertretung der Internationalen Vereinigung Intergeschlechtlicher Menschen (OIl Deutschland) beanstandet vor allem, »dass es sich nicht um eine Wahlmöglichkeit, sondern um eine Vorschrift handelt« (Oll, 2013). Demnach obliege die Definitionsmacht von »weiblich» und »männlich« weiterhin dem medizinischen Diktat. Die Forderung nach einer offenen Geschlechtseintragung gleichberechtigt für alle Menschen bleibe laut Oll weiterhin unerfüllt (vgl. ebd.). 
schaftsgefühls sehr wichtig und regelmäßige Treffen sind für jede_n Einzelne_n von besonderer Bedeutung:

»Wir sind nicht allein, wir sind viele! Die Gruppe ist für uns alle eine Heimat geworden. Wir können uns gegenseitig helfen, mit Problemen fertig zu werden « (SHG Eltern, o.J.).

Das Angebot des Vereins umfasst zusätzlich ein webbasiertes Onlineberatungsangebot, das mit maximaler Sicherheit und Anonymität genutzt werden kann (vgl. IMeV, 2011, S. 8). Dabei besteht sowohl die Möglichkeit einer E-Mail-Beratung als auch eines Einzelgesprächs im Chat. Sogar eine Kontaktaufnahme per SMS ist möglich (vgl. SHG XY-Frauen, o.J.). Ein Forum für intergeschlechtlichen Aktivismus ermöglicht die Kontaktaufnahme zu und den Austausch mit anderen »Betroffen « und bietet Raum für Diskussionen zu unterschiedlichen Themen. ${ }^{43}$ Um auch Gästen die Nutzung des Forums zu gestatten und zugleich weiterhin eine vertrauensvolle Atmosphäre zu gewährleisten, wurden weitere Foren geschaffen, die nur für registrierte und freigeschaltete Usergruppen sichtbar und nutzbar sind (vgl. IMeV., 2010).

\section{Zwischengeschlecht.org}

Zwischengeschlecht.org ist eine Nichtregierungsorganisation in Zürich, die sich auf internationaler Ebene aktiv für Menschenrechte von Inters* einsetzt (vgl. Bauer, 2014, S. 3). Ziel dieses Vereins ist »ein gesetzliches Verbot aller nicht eingewilligten, medizinisch nicht notwendigen Zwangsbehandlungen an Menschen mit > atypischen < körperlichen Geschlechtsmerkmalen « und »die Durchsetzung aller $>$ Menschenrechte auch für Zwitter! $\ll \ll 44$ (ebd.). Dem Recht auf körperliche Unversehrtheit kommt dabei eine besondere Rolle zu (Zwischengeschlecht.org, 2010a).

Seit 2007 engagieren sich Aktivist_innen, die sich aus »Betroffenen «, Angehörigen und solidarischen Unterstüzer_innen zusammensetzen, in den Bereichen Aufklärungs-, Öffentlichkeits- und Lobbyarbeit. Sie unterstützen juristische, völkerrechtliche und politische Initiativen (vgl. ebd.) und prägen den deutschspra-

43 Das Forum scheint aktuell nicht mehr genutzt zu werden. Die letzten Einträge datieren sich auf das Jahr 2011.

44 Der Begriff Zwitter wird hier von Inters* als positive Eigenbezeichnung verwendet. 
chigen Diskurs um Intergeschlechtlichkeit durch öffentlichkeitswirksame Aktionen. Sie begleiten kritisch Gerichtsprozesse und intervenieren durch Proteste bei Tagungen und Kongressen. Ergänzend dazu versuchen sie durch Gespräche verantwortliche Mediziner_innen sowie medizinisches Fachpersonal, Ethiker_innen und Politiker_innen für das Thema »Genitalverstümmelung « zu sensibilisieren (vgl. ebd.). Auch die medizinische Indikation im Fall von Schwangerschaftsabbrüchen bereitet dem Verein hinsichtlich einer gesellschaftlichen Öffnung und Entpathologisierung weiterhin Unbehagen:

»Infolge der zunehmenden Pränataldiagnostik gewinnt zudem die Problematik der seit 1972 zugelassenen selektiven (Spät)-Abtreibungen von Kindern wegen > Gefahr intersexueller Mißbildungen $<$ stetig an Bedeutung $\ll\left(\right.$ Seelenlos ${ }^{45}$, 2012).

Da Eltern intergeschlechtlicher Kinder oft andere Bedürfnisse als erwachsene Inters* formulieren, werden vom Verein Kontakt und Austausch zwischen Elternund Inter*-Selbsthilfegruppen als besonders wichtig beschrieben. Vor allem gehe es darum, dass sowohl Eltern als auch die Gesellschaft im Allgemeinen ein realistisches Bild von der Lebensrealität intergeschlechtlicher Menschen erhalten, um zu wissen, welchen Herausforderungen die Kinder in ihrem konkreten Lebensumfeld ausgesetzt sind. Aufklärung ist dabei außerordentlich wichtig. Aus diesem Antrieb heraus gründete Daniela Truffer, Vereinsvorsitzende_r und ebenfalls Gründungsmitglied von Zwischengeschlecht.org, auch die Elterngruppe intersex.ch (vgl. Truffer, 2011; Freie-radios.net, 2011).

In der öffentlichen Anhörung des Deutschen Ethikrates vom 8. Juni 2011 bezieht Daniela Truffer zur gesellschaftlichen Lebenssituation von Inters* Stellung. Truffer beruft sich dabei auf zentrale Ergebnisse der - hier bereits vorgestellten - Studie des Netzwerks DSD, die zeige, dass in deutschen Kinderkliniken noch immer $\gg 90$ Prozent aller [intergeschlechtlichen] Kinder und Jugendlichen durchschnittlich mehrfach verstümmelt [werden]; die Hälfte der Kinder und 20 Prozent der Jugendlichen werden heute noch gar nicht oder nur unzureichend aufgeklärt « (Truffer, 2011, S. 3). Dabei unterliegen die meisten Formen von Intergeschlechtlichkeit keiner notwendigen medizinischen Indikation. Dennoch würden sie weiterhin als » psychosozialer Notfall « behandelt:

> Chirurgische $>$ Genitalkorrekturen $<$ und $>$ Harnröhrenverlegungen $<$ im Kindesalter sind dagegen - wie die MedizynerInnen immer mal wieder selbst zugeben, [...]

45 Aus Gründen der Anonymität verwendet der_die Sprecher_in das Pseudonym »Seelenlos«. 
medizinisch nicht notwendige, kosmetische Genitaloperationen aus > ästhetischen< oder > psychosozialen< Gründen und werden letztlich nicht für das Kind gemacht, sondern > zur Beruhigung< der meist überforderten und von den MedizynerInnen zusätzlich unter Druck gesetzten Eltern « (Seelenlos, 2012).

In Hinblick auf Ausnahmen für operative Eingriffe wird proklamiert:

»Erlaubt bleiben sollen einzig medizinisch zwingend notwendige Eingriffe, das heißt Eingriffe, deren Aufschiebung irreversible körperliche medizinische Beschwerden zur Folge hätte, zum Beispiel chirurgische Eingriffe zur Behebung von Verschlüssen oder Behinderungen im harnableitenden System und Hormonbehandlungen zum Beispiel bei Cortisolmangel oder zur Aufschiebung vorzeitiger Pubertät « (Truffer, 2011, S. 2).

In einem Radiointerview vom 15.11.2011 wurden die zwei Gründungsmitglieder von Zwischengeschlecht.org nach ihren Aktivitäten und Forderungen im Rahmen ihrer Vereinstätigkeiten befragt. Unter dem Pseudonym »Seelenlos « gibt eine_r der beiden Befragten an, dass »Betroffene « die kosmetischen Genitaloperationen an Kindern als westliche Form der Genitalverstümmelung anklagen (vgl. Freie-radios.net, 2011). Deshalb fordert der Verband, in expliziter Analogie zur weiblichen Genitalverstümmelung (FGM), ein gesetzliches Verbot dieser Operationen. Darüber hinaus plädiert er für ein offenes Erziehungsgeschlecht der Kinder, um ihnen zu ermöglichen, selbstbestimmte Entscheidungen bezüglich medizinischer und hormoneller Eingriffe im Erwachsenenalter zu treffen (vgl. ebd.).

Ein Problem bei juristischen Auseinandersetzungen stellt die Verjährungsfrist dar. Da die meisten Inters* schon im frühen Kindesalter operiert werden, kommt es für sie aufgrund der in der Regel bereits abgelaufenen Verjährungsfrist ${ }^{46}$ oft nicht mehr in Betracht, ihren Anspruch auf Entschädigung juristisch durchzusetzen. Diese Verjährungsfristen stellen Verfahrenshindernisse dar - eine zivilrechtliche Verfolgung bzw. ein zivilrechtlicher Anspruch aufSchmerzensgeld oder Schadensersatz wird dadurch unmöglich. Aus diesem Grund kämpft Zwischengeschlecht.org für eine Aufhebung der Verjährung bzw. für die Verlängerung ihrer Fristen (vgl. ebd.). Die Klage auf Schadensersatz von Christiane Völling gegen den Arzt, der ohne informierte Einwilligung ihre_seine innere weibliche

46 Verjährungsfristen im Falle zivilrechtlicher Anspruchsforderungen belaufen sich in der Regel auf einen Zeitraum von 30 Jahren. 
Anatomie entfernte, war zwar erfolgreich, ist aber bisher ein Einzelfall. Grund für die Zulassung und Annahme der Klage war, dass Völling im Gegensatz zur Mehrzahl der »Betroffenen « erstmalig im Alter von siebzehn Jahren infolge der Feststellung ihrer_seiner Intergeschlechtlichkeit operiert wurde. Wäre er_sie früher dieser Operation unterzogen worden, so wäre die Klage vermutlich an der gesetzlichen Verjährungsfrist gescheitert (vgl. ebd.). Völling bekam erst im Alter von 46 Jahren, also 28 Jahre nach der Operation, Einsicht in ihre_seine Krankenakte und reichte daraufhin Schadensersatzklage gegen den_die behandelnde_n Ärzt_in ein (vgl. ebd.).

Für »Seelenlos « stellt es einen unerlässlichen Faktor ihrer_seiner aktivistischen Tätigkeit dar, vor den Kliniken, die Eingriffe vornehmen, Präsenz zu zeigen. Er_sie ist der Ansicht, dass eine intensivere Aufklärung in der Gesellschaft den medizinisch Handelnden den »Nährboden « entziehe und eine größere Transparenz dazu führe, dass die geschlechtszuweisenden und -vereindeutigenden chirurgischen Praktiken gesellschaftlich weniger toleriert würden (vgl. ebd.). Über die Homepage des Vereins hinaus informiert Zwischengeschlecht.org in einem separaten Info-Blog über aktuelle Geschehnisse und Auseinandersetzungen zum Thema (vgl. Barth et al., 2013, S. 121).

\section{Verein Intersexueller Menschen Österreich}

Der Verein Intersexueller Menschen Österreich (VIMÖ) ist ein neu entstandener Zusammenschluss mit dem Ziel, die Lebensqualität intergeschlechtlicher Menschen zu verbessern und der ihnen gegenüber ausgeübten (strukturellen) Diskriminierung entgegenzutreten. Er wurde am 7. Februar 2014 in Linz gegründet (vgl. Spacefemfm, 2014) und ist der erste Verein, der sich in Österreich explizit für die Belange von Inters* einsetzt. Der VIMÖ ist zugleich Mitglied der Internationalen Vereinigung intergeschlechtlicher Menschen (Organisation Intersex International, OII Austria).ZZudem sind Vetreter_innen des Vereins auch Initiator_innen der Plattform Intersex Österreich, die eine Vernetzung mit Expert_innen verschiedener Disziplinen ermöglicht (VIMÖ, 2014). Der VIMÖ nimmt somit eine maßgebliche Position im deutschsprachigen Diskurs um Intergeschlechtlichkeit ein. Als Gründungsmitglieder lassen sich drei zentrale Personen benennen, deren Selbstzeugnisse und aktivistische Tätigkeiten hier kurz skizziert werden.

Alex Jürgen ist durch den Film Tintenfischalarm (Scharang, 2006a) aus dem Jahr 2006 bekannt geworden. Drei Jahre wurde Alex Jürgen von der_dem Regisseur_in und Moderator_in Elisabeth Scharang mit der Kamera begleitet, die 
damit - zunächst unbeabsichtigt - seine Transition zum intersexuellen Mann dokumentiert. So wird aus einer geplanten Dokumentation über Intergeschlechtlichkeit ein $\gg$ Live-Experiment $\ll$ :

»Es beginnt die Zeit der Selbstbeobachtung, ein Echtzeitexperiment: Die Auswirkungen der männlichen Hormone, die Alex in Form einer Creme zu sich nimmt, werden von uns genau studiert. Ich habe das Gefühl, einem Mann beim Wachsen zuzusehen! (Scharang, 2006b, S. 5).

Alex Jürgen beschreibt diese Phase in seinem Leben als entscheidenden Schritt zur Lebensbejahung, die ihn auch darüber hinaus aktiv werden lässt:

» 10 Jahre und eine Leukämieerkrankung später beschloss ich, mein Leben weiterhin als intersexueller Mann fortzuführen. Ließ mir die hormongewachsenen Brüste amputieren und änderte meinen Geschlechtseintrag in den Papieren in MÄNNLICH. Seitdem kämpfe ich für das Recht auf einen unversehrten Körper und versuche auf meine Weise, die Thematik Intersexualität an die Öffentlichkeit zu bringen. Mit dem Kinofilm TINTENFISCHALARM startete ich 2006 mein Outing und lebe bis heute völlig offen in einer Gesellschaft, in der es nur Männer und Frauen geben darf, und bin glücklich, nach all den Jahren endlich der Mensch sein zu können, der ich eben bin. Nicht Mann, nicht Frau. Einfach ICH!« (Jürgen, 2013).

Auch Rasa Humer spürte über die Auseinandersetzung mit der eigenen Geschichte den Impuls, als Aktivist_in tätig zu werden. Ähnlich wie Alex Jürgen begann auch Humer mit der Einnahme von Testosteron und ließ die Öffentlichkeit an dem einsetzenden Prozess teilhaben. Als Blogger_in teilt Rasa Humer eigene Erfahrungen im Internet und versucht so, mit der eigenen Geschichte, als Vorbild in zu agieren (Humer, 2014). Die Entscheidung, sich einer verweiblichenden Operation zu unterziehen, sehe er_sie mittlerweile nicht mehr als freiwillig und selbstbestimmt an. Vielmehr sei er_sie damals zu wenig aufgeklärt worden, um eine klare Position zu entwickeln. Das Motiv für die öffentliche Darstellung der persönlichen Geschichte ist es, eigene Erlebnisse und Erfahrungen nachvollziehbar und sowohl den Einfluss von Hormonen als auch den Prozess der Vermännlichung wahrnehmbar zu machen. Für Humer ist es eine Art Experiment, die Öffentlichkeit teilhaben zu lassen. Gleichzeitig beschreibt er_sie, dass das stetige Wiedergeben der eigenen Erfahrungen zwar einerseits wichtig auch persönlich wichtig - ist, aber andererseits zeitweise viel Energie erfordert. 
Dennoch beschreibt Humer, dass er_sie die Überwindung dieser Angstschwelle - der Furcht vor (negativer) Reaktion - als außerordentlich befreiend und »empowernd $\ll{ }^{47}$ erlebt habe und durch diese Herangehensweise das historisch auferlegte Schweigegebot breche (vgl. Spacefemfm, 2014).

Das dritte Gründungsmitglied ist Gorji Marzban. Marzban hat bereits 2010 die Oriental Queer Organisation Austria (ORQOA) mitbegründet, die sich gegen Gewalt, Trans*- und Homophobie und für die Rechte und Anerkennung geflüchteter Menschen aus der arabischen LGBTIQA ${ }^{48}$-Community einsetzt. ORQOA gehört darüber hinaus dem internationalen Netzwerk LGBTIQ-Welcome an (vgl. ORQOA, 2012). Marzbans Einstellung zu Gender spiegelt ihre_seine Einstellung zum Leben wider:

$\gg$ Ich bin ein intersexueller Mensch und muss es nicht beweisen. Der Mensch ist in erster Linie Geist und nicht Geschlecht. Diesen Geist kann man individuell in jedes Gefäß geben. Er bleibt sowieso erhalten « (Marzban, 2012).

Als übergeordnete Ziele von VIMÖ nennt der Verein, Intergeschlechtlichkeit zu enttabuisieren und die damit einhergehenden alltäglichen und gesellschaftlichen Probleme zu bearbeiten. Das Bewusstsein hinsichtlich der Existenz von Inter* soll befördert und Inter* als Varianz anerkannt statt pathologisiert werden. Eine öffentliche Auseinandersetzung mit dieser Thematik soll angeregt und zur aktiven Mitgestaltung mobilisiert werden (vgl. ebd.). Rasa Humer profitiert stark von dieser Vernetzungs- und Vereinsarbeit, da sie ihr_ihm ein Gefühl von Empowerment gebe (vgl. Spacefemfm, 2014).

47 Im allgemeinen Sprachgebrauch wird Empowerment als das Zurückgeben von Kraft verstanden. Es geht nicht nur um ein Teilnehmen, es geht um ein Teilhaben-Lassen (vgl. Sickendiek et al., 1999, S. 71), einen Zuwachs an persönlicher und kollektiver Handlungsfähigkeit (vgl. Stark, 2004, S. 535), eine Verantwortungsübernahme für die Gestaltung eigener Lebensräume und sozialer Zusammenhänge und die Beförderung von Resistenz gegen Formen und Bedingungen sozialer Ungerechtigkeit (vgl. Bamberger, 2004, S. 773). Das Hervorheben der eigenen Durchsetzungsfähigkeit muss in einen Rahmen ethisch-moralisch begründbaren Handelns eingebettet sein, quasi einer gleichzeitigen Anerkennung eines Gemeinwohls, um der Gefahr einer Instrumentalisierung vorzubeugen. Für Beratung allgemein macht dies die Herausarbeitung spezifischer Teilhabestrategien notwendig (vgl. Sickendiek et al., 1999, S. 72).

48 LGBTIQA (engl.) bzw. LSBTTIQA ist eine Abkürzung für den Personenkreis geschlechtlicher Besonderheiten und steht für lesbisch, schwul, bisexuell, trans*/transident, transsexuell, inter* und queer. Das »A« bezeichnet in diesem Zusammenhang Asexualität und wird in dieser Arbeit ebenso wie andere Identitäts- und Begehrensformen als gleichberechtigter Teil von Sexualität verstanden. 
Aus der Vereinssatzung von VIMÖ geht hervor, dass sich die Mitglieder für die freie Wahl der Geschlechtsidentität und des Geschlechtseintrags einsetzen. Als Basisziele werden zudem die Wahrung und Einhaltung der universalen Menschenrechte formuliert, insbesondere das Recht auf seelische und körperliche Unversehrtheit sowie der Schutz vor Diskriminierung (vgl. VIMÖ, o.J.). Sie forcieren die Errichtung von Netzwerken ebenso wie die allgemeine Aus- und Weiterbildung von Fachkräften aus sozialen und pflegerischen Berufen sowie aus dem medizinisch-psychologischen Sektor. Dafür fordern sie Unterstützung und Förderung, um Lehrmittel bereitstellen, geeignete Broschüren erarbeiten und fach- und themenspezifische Schulungen durchführen zu können (vgl. ebd.). Ein Flugblatt des Vereins informiert über Selbstverständnis, Angebote und Forderungen. Ziel soll es sein, diesen Infoflyer landesweit auf Geburtenstationen, in gynäkologischen und urologischen Einrichtungen sowie an queeren Treffpunkten auszulegen und zu verteilen (vgl. Spacefemfm, 2014). Verschiedene Aufgaben zählen zum Tätigkeitsprofil des Vereins Intersexueller Menschen Österreich:

Es soll eine aktive Selbsthilfegruppe, die sich in regelmäßigen Abständen trifft, etabliert und geleitet werden. Die Teilnahme an den derzeit monatlich stattfindenden Treffen ist auch hier ausschließlich den intergeschlechtlichen Personen möglich. Diese Exklusivität wird als besonders wichtig erlebt. In Abgrenzung zur Trans*-Thematik und zur schwul-lesbischen Thematik wird Inter* als selbstständiger »Problembereich « begriffen. ${ }^{49}$ Das gelte ebenso für die Selbstvertretung als politische Dimension, um Forderungen an Politik und Medizin zu stellen (vgl. ebd.). In einem Radio-Interview mit dem Sendungstitel »Hoch die intersexuelle Solidarität«, bestätigt Rasa Humer diese Auffassung. Ihrer_seiner Ansicht nach will sich die Inter*-Community nicht länger fremdbestimmen lassen, sondern selbst aktiv werden und aufzeigen, was Inters* brauchen und was nicht. Als Vertreter_in und Mitbegründer_in des VIMÖ äußert sich Humer klar zu einer Abschaffung von kosmetischen Operationen an Kindern und der Entfernung funktionaler Keimdrüsen, die die Notwendigkeit der lebenslangen Einnahme von Hormonen zur Folge hat (vgl. ebd.). Chirurgische Interventionen an den intergeschlechtlichen Genitalien werden von Humer als »Genitalverstümmelungen « bezeichnet und als Menschenrechtsverletzung angesehen. Da sie medizinisch als Heilbehandlung propagiert werden, seien diese jedoch gesellschaftlich toleriert

49 Humer hebt dennoch ausdrücklich als positiv hervor, dass sich innerhalb der Plattform Intersex auch Nicht-»Betroffene« solidarisch engagieren. Seiner_ihrer Ansicht nach ist das besonders auch unter dem Aspekt der eigenen Traumatisierungen wichtig, da »Betroffene« oft nur begrenzt einsatzfähig seien und dadurch immer wieder ihre eigenen Grenzen erlebten (vgl. Spacefemfm, 2014). 
(vgl. ebd.). Weiterhin führt Humer an, dass die medizinische Forschung und Praxis glaube, dass eine gesunde psychosexuelle Entwicklung nur möglich sei, wenn die Genitalien als eindeutig » männlich « oder » weiblich « erkennbar seien. Humer hingegen vertritt diesbezüglich eine grundsätzlich entgegengesetzte Position. Er_sie erläutert, dass es nur dann zu einer »gesunden « psychosexuellen Entwicklung kommt, wenn keine geschlechtszuweisenden und -vereindeutigenden Eingriffe im Kindes- und Jugendalter vollzogen werden und man nicht das Gefühl vermittelt bekommt, »falsch « zu sein (vgl. ebd.). In Form von Einzeloder Familienberatungen stellt der Verein verschiedene Angebote zur Verfügung. Diese richten sich vor allem an Inters* selbst, aber auch an deren Eltern und Angehörige (vgl. ebd.). Darüber hinaus verfügt der Verein über Weitervermittlungskompetenzen, um an andere psychosoziale oder medizinische Einrichtungen $\mathrm{zu}$ verweisen und so Inters* und deren Familien bei ihrer Suche zu unterstützen (vgl. VIMÖ, 2014). Eine breit gefächerte Bildungs- und Öffentlichkeitsarbeit in Form von Aufklärungsworkshops an Schulen, bei Vereinen und Behörden sowie Schulungen für beratendes Fachpersonal (vgl. Spacefemfm, 2014) soll zukünftig diese praktische Arbeit begleiten (vgl. VIMÖ, o.J.). Zudem will das Team des VIMÖ Offenheit für Einladungen innerhalb von Arbeitskontexten (Soziale Arbeit, Pädagogik, Medizin) signalisieren, um aus der »Betroffenenperspektive « zu berichten, aufzuklären, und Informationen bereitzustellen (vgl. Spacefemfm, 2014). Durch die Organisation politischer und kultureller Veranstaltungen (Vorträge, Lesungen, Ausstellungen, Filme) sollen Möglichkeiten entstehen, einen großen Kreis verschiedener Interessent_innen zu erschließen. Zudem plant der Verein, periodisch Publikationen zu veröffentlichen, Veranstaltungen zu dokumentieren und gegebenenfalls an wissenschaftlichen Studien und Forschungsprojekten teilzunehmen oder diese zu initiieren (vgl. ebd). Momentan liegt die Hauptaktivität in der Öffentlichkeitsarbeit und der Akquise von Fördermitteln (vgl. ebd.).

\section{Ausgangspunkt subjektiver Betrachtungen: Ergebnisse qualitativer Expert_innen-Interviews}

Sowohl die Stellungnahmen der »Betroffenenverbände « als auch die zuvor in den Studien geäußerten Problemlagen intergeschlechtlicher Personen deuten auf eine Leerstelle im aktuellen Beratungsangebot hin. Gestützt wird diese These zusätzlich durch zwei Interviews, die im Vorfeld dieser Veröffentlichung durchgeführt wurden. Mittels leitfadengestützter qualitativer Interviews wurden zwei 
Expert_innen der Inter*-Bewegung nach ihren individuellen Beratungserfahrungen befragt. ${ }^{50}$ Die beiden Aktivist_innen waren Lucie Veith, Vorsitzende_r des Bundesverbandes Intersexuelle Menschen e.V., sowie ein_e Vertreter_in der Kampagne Dritte Option ${ }^{51}$. Das Erkenntnisinteresse galt der Frage, wie nach Ansicht der Aktivist_innen eine professionelle Beratung konzipiert sein muss, um von Inters* in Anspruch genommen zu werden. Dabei wird von folgenden zwei Grundannahmen ausgegangen: Einerseits wird bei der Frage nach Beratung bzw. Beratungserfahrung zunächst nur an medizinische Beratung gedacht bzw. nur diese benannt. Andererseits werden diese Vorerfahrungen mit beratenden medizinischen Institutionen als negativ erlebt. Anknüpfend an persönliche Berührungspunkte mit konkreten Beratungssituationen bestätigt der_die Vertreter_in der Kampagne Dritte Option:

»[Es war] dann halt so, dass ich quasi zu einer Frauenärztin gegangen bin, weil sich mein Körper halt nicht so ganz so entwickelt hat, wie das halt erwartet wird, wenn das ein weiblicher Körper ist. Also, das heißt halt ganz konkret, dass ich halt nicht, also mit 15 war das glaub ich oder so, noch nicht meine Tage bekommen habe, dass ich nicht so eine Brustentwicklung hatte wie andere, dass meine Stimme irgendwie was tiefer war und halt solche Sachen alles. Und das war auf jeden Fall eine Erfahrung, die halt für mich ziemlich krass war, also weil, ich meine, ich glaube das ist für alle Leute unangenehm, so diese Situation, bei so einer Frauenärztin irgendwie zu sein und da so an so intimen Sachen, dass die sich das halt alles anguckt und so. Aber ich habe halt sofort gemerkt, dass die halt einfach irgendwie schockiert war quasi. Also die hat halt dann gesehen, dass auch meine/also dass auch sozusagen von irgendwelchen erwarteten inneren Organen das halt nicht so aussah wie/also dass

50 Als Transkript respektive Transkription wird die Übertragung eines gehörten Textes z. B. einer Sprachaufnahme in die Schriftsprache z.B. in einen geschriebenen Text verstanden (vgl. Kohler, 1995, S. 14). Die Interview-Transkripte der vorliegenden Arbeit werden in leicht bereinigter Form zitiert; dies betrifft vor allem die Grammatik. Bei der Auswertung der Interviews hinsichtlich dieses Abschnitts liegt der Fokus darauf, »was" gesagt wird, und weniger darauf, »wie« etwas gesagt wird. Die Schrägstriche innerhalb der Zitate bilden dabei die Ist-Aussprache des gehörten Textes ab und verdeutlichen Abbrüche im Redefluss. Der Satz wird nicht zu Ende gesprochen und ggf. durch weitere Gedanken der_s Sprecher_in an dieser Stelle ersetzt. Aus Gründen der Forschungsmoral sind die Interview-Transkripte nicht öffentlich zugänglich.

51 Die Kampagne »Dritte Option. Für einen dritten Geschlechtseintrag « kämpft auf dem juristischen Weg um die Anerkennung einer dritten Wahlmöglichkeit im Personenstandsgesetz und hat hierfür am 28. Juli 2014 Klage beim Amtsgericht Hannover eingereicht (vgl. Kampagne Dritte Option, 2014a). 
die Eierstöcke irgendwie ganz klein waren und die Gebärmutter und so was alles, und das ist halt irgendwie so ein Gefühl, was natürlich schon da geblieben ist und die hat dann noch irgendwelche Sachen von genetischem Defekt [...] geredet, und ich bin dann auch nicht mehr hingegangen danach, aber das war ja quasi die erste Person, die [...] mir da irgendwas über mich eigentlich mitgeteilt hat, aber halt auf eine Art und Weise, die es mir halt sehr schwer gemacht hat, mich da weiter mit zu beschäftigen « (Kampagne Dritte Option, 2014b, S. 6f.).

Nicht selten erscheinen einzelne Untersuchungen aus medizinischer Perspektive als nicht ausreichend. Stattdessen wird ein langer und mühsamer Prozess der diagnostischen Klärung in Gang gesetzt. ${ }^{52}$ Dieser bedeutet für die untersuchten Personen unter Umständen, dass sie unangenehme Situationen wiederkehrend durchleben, was retraumatisierend wirken kann. Ein anderer Aspekt ist die damit verknüpfte medizinische (Zwangs-)Behandlung, die einerseits durch Hormongabe und -substitution, andererseits in Form chirurgischer Eingriffe erfolgen kann:

» [Ich] war dann bei so einem Endokrinologen, der hat dann auch festgestellt, dass ich ein X-Chromosom halt habe, und da war es dann halt aber ganz klar, sozusagen, dass ich jetzt irgendwie weibliche Hormone bekommen muss so. Und ich habe die dann halt auch genommen, so, wo ich halt jetzt im Nachhinein sagen würde, dass die eigentlich ja so Veränderungen an meinem Körper gemacht haben, die ich eigentlich gar nicht will, so, oder wo ich so ein bisschen traurig jetzt manchmal darüber bin quasi, und da wurde halt aber einfach gar nicht sozusagen wirklich viel darüber geredet, dass es halt vielleicht auch die Möglichkeit geben würde, andere Hormone zu nehmen. Das war halt irgendwie klar, du bist halt eigentlich weiblich, und du musst halt irgendwie jetzt so dann was nehmen oder machen, damit es halt auch so mehr da reinpasst. Und deswegen, das war schon so eine sehr nicht so positive

52 In der Literatur und in Selbstaussagen werden insbesondere zwei Zeitabschnitte benannt, in denen die Intergeschlechtlichkeit eines Menschen sichtbar wird. Ein phänotypisches Bild der äußeren Geschlechtsmerkmale kann möglicherweise bereits zum Zeitpunkt der Geburt auf eine »Uneindeutigkeit« hinweisen. Nicht selten bleiben intergeschlechtliche Kinder bis zum Eintritt in die Pubertät »unentdeckt«. Aufgrund verschiedener hormoneller, chromosomaler sowie gonadaler Einflüsse kann es dann zur Ausbildung sekundärer Geschlechtsmerkmale kommen, die medizinisch als »nicht typisch « gelten. Zum Beispiel kann ein ehemals »weiblich« gelesener Körper »vermännlichen« oder umgekehrt. Im Fall Christiane Völlings waren es körperliche Beschwerden, die zur »Entdeckung « der Intergeschlechtlichkeit führten. Die drei genannten Möglichkeiten stellen jedoch lediglich eine Auswahl an denkbaren Vorkommnissen dar. Die (medizinisch-psychologische) Konsequenz bleibt letztlich dieselbe. 
Form von Beratung, sage ich jetzt mal. Also, und natürlich, [...] der Endokrinologe, der war schon dann irgendwie netter und so, und der wollte mir jetzt nicht das Gefühl geben, dass ich irgendwie was total ganz Schlimmes bin oder so, aber, ja, es war halt tatsächlich schon auch einfach so ein Mediziner, wo ich mich jetzt nicht total viel/mit dem jetzt über meine persönlichen Gefühle von Geschlechtsidentität irgendwie so reden konnte « (Kampagne Dritte Option, 2014b, S. 7).

Das folgende Zitat belegt eindeutig, dass es durch medizinische Behandlungen zu schweren Grenzverletzungen kommen kann. ${ }^{53}$ Es zeigt, dass die im Behandlungsprozess ausgeübte Gewalt oft bewusst nicht eindeutig erwähnt oder sogar bagatellisierend dargestellt wird:

»[Ich] habe in der Diagnostik [...] ein Trauma erlitten und durch diese ganzen Übergriffe, durch diese vielen Menschen, die mich untersucht haben, zum Beispiel, dass man dort auf der einen Seite eine Mauer des Schweigens um mich gebaut hat und auf der anderen Seite/und auch [...] der Tabuisierung, [mit] mir darüber zu reden, das war ja fast Einzelhaft, Einzelzimmer, natürlich alles bestens, alles wunderbar, die wollten mir alle was Gutes tun, aber niemand hat mit mir darüber geredet, was da passiert, und trotzdem sind sie mit 24 Leuten ins Zimmer gekommen, ich durfte mich dann freimachen, und dann haben mich drei Leute am gleichen Tag im Bett befingert. Die haben sich nicht mal, zwei von denen haben sich nicht mal einen Gummihandschuh übergezogen. Und das war für mich/ich hatte schon [...] einen sexuellen Übergriff vorher erlebt/, das war für mich eine Vergewaltigung. Und dabei ist meine Emotion, ja, in mir gestorben « (Veith, 2014, S. 17f.).

Das Resultat dieser gewaltvollen Erfahrung ist, dass Menschen misstrauisch gegenüber beratenden Institutionen geworden sind und selbst neuen Beratungsangeboten gegenüber Vorbehalte haben:

»Ich glaube, ich hatte immer schon so ein bisschen so eine Skepsis vor Beratung, halt so dieses Ding, ich habe halt Angst quasi, so ein bisschen, das zu sagen, und das hat/ich habe das vielleicht auch eher gelernt, dass man so denkt, man muss mit seinen Sachen so ein bisschen alleine klarkommen oder so, und das ist ein bisschen

53 Mit Sicherheit kommt es nicht in allen Fällen medizinischer Behandlung zu schweren Grenzverletzungen. Diese können ebenso gut in anderen Bereichen innerhalb des Hilfesystems erfolgen. Dennoch ist an diesem Punkt wichtig zu verdeutlichen, dass diese Grenzverletzungen nicht selten vorkommen, obwohl sie in der Literatur oft nicht als solche gekennzeichnet bzw. explizit benannt werden. 
so nicht so leicht, fällt sozusagen sich selber so/also so eine Form von Schwäche vielleicht irgendwo auch zu zeigen « (Kampagne Dritte Option, 2014b, S. 11).

Diese Ansicht stellt eine besondere Herausforderung für neu zu etablierende Angebote dar, die von Inters* auch unterstützt und selbst in Anspruch genommen werden. Es bedarf deshalb eines hohen Maßes an Sensibilität und Kenntnis über die Lebenssituationen intergeschlechtlicher Menschen, um neue Konzepte zu entwickeln. Diesen Aspekt greift ebenso Lucie Veith im Interview auf:

» [Ich] glaube, das ist [...] eine Gefahr, die auch unterschätzt wird, diese retraumatisierenden Gespräche und diese Krisen, die das auslöst, die so eine Beratung auslösen kann. Darauf muss man sehr sorgsam achten. [...] Ich [...] möchte nicht, dass irgendjemand von der Brücke springt oder auf das nächste Dach geht. Denn, [...] das muss ich wissen, dass das passieren kann. Deswegen auch diese hohe Verantwortlichkeit, und ich sage lieber, [...] lass da nicht jeden Baum zur Beratungsstelle werden, sondern, [...] ja, es ist eine besondere Verantwortung, die man da trägt. Ich glaube, [...] keine Beratung ist besser als eine schlechte Beratung « (Veith, 2014, S. 19).

Beide Interviews bestätigen die von den »Betroffenenverbänden « artikulierten Probleme mit dem aktuellen Beratungsangebot in Deutschland. Zum einen benennen die Interviewten einen grundlegenden Mangel an beratenden Fachkräften, zum anderen eine zu starke Fokussierung auf den medizinischen Bereich. Während der_die Vertreter_in der Kampagne Dritte Option aus einer queerfeministischen Position heraus argumentiert, verknüpft Lucie Veith ihre_seine persönliche Erfahrung mit der praktischen Beratungstätigkeit im Rahmen der Selbsthilfe. Beide Interviewpartner_innen artikulieren Wünsche und Forderungen hinsichtlich inhaltlicher und methodischer Ausrichtung von Beratungsangeboten.

»Also ich denke, dass ein weiterer ganz wichtiger Punkt ist eben tatsächlich, dass man [...] Inter*-Beratung immer, immer [...] multidimensional denken muss und auch immer klären muss, auf welcher Basis unterhalten wir uns jetzt eigentlich? Worum geht es eigentlich? Geht es da um Personenstand? Und geht es darum, wie Leute dich wahrnehmen und wie du dich wahrnimmst, oder meinst du das eher in Bezug auf deine sexuelle Orientierung, also auch auf deine sexuelle Identität, wie siehst du dich, in [...] welcher Rolle siehst du dich, und in welcher Rolle wärst du gerne, und kann ich das erreichen und wie, und da aber immer das Defizit rausnehmen, das ist die Kunst dabei. Das ist nie etwas, auch den Ist-Zustand nie als Defizit 
wahrzunehmen, denn, ganz ehrlich, [...] meistens haben wir es ja mit Opfergruppen zu tun. Menschen, die meistens medizinische Interventionen hinter sich haben, die sich [...] a) nicht selbst entschieden haben und b) bei denen es jede Art von informierter Einwilligung nicht gegeben hat « (Veith, 2014, S. 13).

Auf die Frage, welche Assoziationen sich hinsichtlich einer Verbindung von Inter* und Beratung ergeben, betont der_die Vertreter_in der Kampagne Dritte Option, dass eine Berücksichtigung der Wünsche und Bedürfnisse der beratenen Person elementar sein muss:

»Dazu fällt mir ein, dass es das halt irgendwie eigentlich noch so kaum gibt, quasi einfach, dass es halt auch allgemein eher bei ganz vielen Stellen, wo dann, das Thema vielleicht auftaucht, sehr viel Unwissenheit und Unwissen zu dem Thema gibt, und ja, dass halt auch gerade es eben total wichtig ist, dass eine Beratung halt möglichst, ja, möglichst offen damit umgeht, was die Wünsche und Bedürfnisse von Leuten sind, und nicht davon ausgeht, diese schon zu kennen « (Kampagne Dritte Option, 2014b, S. 5).

In einem weiteren Vorschlag wird ein Bezug zum Peer-Involvement hergestellt. Auch Lucie Veith vertritt diesen Ansatz, da ihrer_seiner Meinung nach die »Selbsthilfe [...] immer noch viel, viel dichter an die Menschen ran[kommt] als eine Beratungsstelle zum Beispiel« (vgl. Veith, 2014, S. 2). Diese Aussage basiert auf der Annahme einer gemeinsam geteilten Erfahrung, die Sicherheit vermittelt und das Gefühl unterstützt, verstanden und ernst genommen zu werden. Deshalb sieht Veith den Erfolg von Beratung nur in Ergänzung zur Selbsthilfe. Dabei steht der Wahrung professioneller Distanz als Grundsatz der Sozialen Arbeit eine emotionale Involviertheit nicht nur gegenüber, sondern bildet eine Schnittstelle:

»Ich glaube, professionelle Beratung ist dann gut, wenn sie halt tatsächlich nicht so abgehoben von den/also wenn die Menschen, die da selber Beratung machen, vielleicht auch nicht so weit entfernt sind von den Menschen, die Beratung benötigen, und ich weiß, dass dann ganz oft in der Sozialen Arbeit eher gerade davon geredet wird, dass so diese professionelle Distanz irgendwie ganz wichtig ist, und ich kann mir auch vorstellen, dass das für unterschiedliche Menschen individuell anders ist, aber ich finde es zum Beispiel viel einfacher, Unterstützung zu bekommen, wenn eine andere Person sich gegenüber mir auch irgendwie als Mensch zeigt und nicht nur als so irgend so eine Instanz, weil es dann einfach für mich einfacher ist, vielleicht mich zu öffnen. Und ja, halt eine Beratung, die irgendwie parteiisch 
ist, also die halt auch meine Bedürfnisse, die ich da vielleicht äußere, jetzt nicht unbedingt als erstes infrage stellt, das fände ich, glaube ich, wichtig, und ja, ansonsten ist es vielleicht wirklich auch einfach unterschiedlich, worum es jetzt gerade geht, also wenn es jetzt aber wirklich Beratung ist, dann auch schon was, was vielleicht eher Zielorientiertes ist, also, was dann auch mich als Person, die vielleicht Beratung sucht, dabei rausholt, überall nur alles [als] Problem zu sehen, sondern so ein bisschen versucht, die Sachen zu fokussieren, wo kann man jetzt konkret was machen und wie sieht das dann aus « (Kampagne Dritte Option, 2014b, S. 3f.).

Hinsichtlich bestehender Angebote könnte ebenso die Ansicht vertreten werden, dass aufgrund der Aktivitäten der Selbsthilfegruppen bereits ein ausreichendes Beratungsangebot als Pendant zum medizinischen Angebot existiere. Diesbezüglich betont Lucie Veith »einen Riesen-Unterschied, weil ich in der Beratung eigentlich verantwortlich für das bin, was ich da auslöse bei dem Menschen. In der Selbsthilfe bin ich das nicht « (vgl. Veith, 2014, S. 10). Die Selbsthilfe kann demnach nicht als ausschließliche Beratungsinstanz dienen. Veith führt weiterhin an:

»Wenn ich etwas [...] professionell als beratend anbiete, dann ist es so/muss ich wissen, was ich tue, in der Selbsthilfe muss ich das nicht, da darf ich es einfach mal geschehen lassen. Da passieren ja auch, so/aus der Gruppe heraus, Dinge, die kann man eigentlich gar nicht erklären, aber die waren da mal dran « (Veith, 2014, S. 11).

Daraus resultiert die Notwendigkeit, eine fachspezifische Inter*-Beratung zu konzipieren, die sich entweder als Schnittstelle zwischen Medizin und Selbsthilfe etabliert oder einen eigenständigen Platz innerhalb der Triade von Selbsthilfe, Beratung und Medizin einnimmt.

Als Resümee der Expertise der Interviewten kann festgehalten werden: Beide Befragten sehen den Beratungsbedarf durch das aktuelle Angebot als nicht ausreichend gedeckt an. Verschiedene Grundsätze, die von den Interviewten als notwendig herausgearbeitet werden, seien nur ansatzweise installiert und umgesetzt. Die vier wesentlichen Prinzipien einer positiven Beratung müssten Offenheit, Akzeptanz, Parteilichkeit und Zielorientierung sein (vgl. Kampagne Dritte Option, 2014b, S. 4). Die Schaffung einer angenehmen Beratungsatmosphäre ist dabei unabdingbar. Es besteht ein starkes Bedürfnis nach Zeit, um in Ruhe erzählen zu können und dabei das Gefühl vermittelt zu bekommen, dass jemand unvoreingenommen zuhört (vgl. ebd.). Berater_innen wird seitens der Interviewten empfohlen, einen offenen Ansatz zu verfolgen und nicht zu versuchen, 
starre und vorgefertigte Konzepte auf die Hilfe suchenden Personen anzuwenden (vgl. ebd., S. 5). Beratung muss flexibel, personenzentriert und bedürfnisorientiert sein. Die geschilderten Lebensumstände und Realitäten der jeweiligen Person müssen innerhalb der Beratung Berücksichtigung finden. Dazu ist es notwendig, dass die Beratenden einerseits Kommunikationsfähigkeiten und Kenntnisse der Gesprächsführung erwerben, um den Hilfesuchenden empathisch zu begegnen, und anderseits ebenso über medizinisch-biologisches Fachwissen verfügen, um die Situation auch in dieser Hinsicht einschätzen sowie professionell und adäquat reagieren zu können (vgl. ebd., S. 26). 


\section{Aspekte einer "gerechten" Inter*-Beratung}

Der folgende Abschnitt versteht sich als Versuch, bestimmte Inhalte und Bereiche von Beratung so zusammenzustellen, dass sie den Ansprüchen von Pluralität, Vielfalt und Diversität auf unterschiedlichen Ebenen, durch verschiedene Inhalte und anhand diverser Formen gerecht und praktisch handhabbar werden. Dabei greife ich auf einen wertvollen Fundus bereits existierender Theorien und Praxisfelder von Beratung zurück und unterziehe diese einer Vorauswahl. Zunächst stelle ich Zugänge zu Beratung im Allgemeinen vor. Im Anschluss daran arbeite ich den Ansatz der psychosozialen Beratung als maßgebend für die vorliegende Veröffentlichung heraus und erläutere ihn in seinen Grundzügen. Aus dem Pool der Methoden für die allgemeine Beratungsarbeit wähle ich unter dem Aspekt der Inter*-Beratung solche Vorgehensweisen und Handlungsansätze aus, die mir als besonders geeignet für die praktische Beratungsarbeit mit der Zielgruppe Inter* erscheinen. Bewährtes wird in diesem Zusammenhang aufgegriffen, neu kontextualisiert und an den Beratungsauftrag angepasst. Es bleibt immer wieder aufs Neue zu prüfen, welcher konkrete Beratungsansatz, welche Methode der jeweiligen Beratungssituation angemessen erscheint und als zielführend empfunden wird. Die Sicht auf und die Handhabung von Beratung muss dabei immer flexibel bleiben. Eine einzig richtige inter*-spezifische Beratung kann es nicht geben. Zu divers sind die Kontexte, in denen sich Menschen bewegen. Obwohl alle hier adressierten Personen eint, dass sie inter* sind, lässt dies keine Rückschlüsse auf die vermeintliche Homogenität einer Gruppe zu. Lebensumstände und -realitäten können sich aufgrund ähnlicher medizinischer oder anderer Erfahrungen, die in engem Zusammenhang mit der Intergeschlechtlichkeit stehen, zwar gleichen, dennoch existiert eine Vielzahl anderer Aspekte, die nicht wegen der Intergeschlechtlichkeit, sondern beispielsweise aufgrund struktureller Probleme schwer zu bewältigen sind: 
» [Die] meisten intersexuellen Menschen leiden nicht darunter, dass sie intersexuell sind, sondern [...] weil das aufgrund [...] des Umgangs von außen immer defizitär gesehen wird « (Veith, 2014, S. 3).

Dieser Ansicht schließt sich auch Phoebe Hart an und konkretisiert:

»Durch die vielen Gespräche mit intergeschlechtlichen Männern und Frauen in den letzten 10 Jahren bin ich zu der Überzeugung gelangt, dass die meisten kein Problem mit ihrer Intergeschlechtlichkeit haben, aber sehr wohl mit den damit einhergehenden $>$ gesellschaftlichen $<$ Schwierigkeiten. Intergeschlechtliche Menschen leiden unter sozialer Stigmatisierung, darunter, als > anders < oder > abartig < gesehen zu werden und von Ärzt_innen und Familienmitgliedern entsprechend behandelt zu werden. Medizinische Behandlungen tragen wesentlich zu dem Gefühl der sozialen Minderwertigkeit und Scham bei: Kosmetische Chirurgie soll abweichende Körper normalisieren, indem sie intergeschlechtliche Personen entweder männlich(er) oder weiblich(er) macht. Faktisch stellt eine solche Pathologisierung eine gesellschaftliche Verschiebung dar, hin zu der Absonderung der intergeschlechtlichen Personen von der normalen Bevölkerung« (Hart, 2013, S. 84).

Wie es Rosa von Praunheim bereits 1970 in ihrem_seinem Film Nicht der Homosexuelle ist pervers, sondern die Situation, in der er lebt ${ }^{54}$ für Homosexualität formulierte (vgl. Praunheim, 1970), so handelt es sich auch bei Intergeschlechtlichkeit primär um ein soziales »Problem« (vgl. Wiesendanger, 2004, S. 245). Sowohl im Umgang mit homosexuellem Begehren als auch im Fall von Intergeschlechtlichkeit scheint die Gesellschaft an Grenzen zu stoßen (vgl. ebd.).

Ebenso kann es vorkommen, dass Inters* Beratung und Unterstützung suchen, ohne jemals ihre Intergeschlechtlichkeit als Teil des Beratungsbedarfs zu sehen. Es ist daher sehr wichtig, von Beginn an den konkreten Auftrag zu klären.

\section{Allgemeine Beratungszugänge und -formate}

Mit dem Begriff »Beratung« werden gesamtgesellschaftlich unterschiedliche Vorstellungen verknüpft, die oft an Erwartungen eines bestimmten Verhaltens, einer Vorgehensweise oder des inhaltlich Gesagten gekoppelt sind und nicht zu-

54 Siehe hierzu http://www.rosavonpraunheim.de/werke/rosafilme/70homo/w_pervers_1. html (Zugriff: 04.08.2014). 
letzt zu missverständlichen Interpretationen führen. Engel et al. (2004) verweisen auf zwei gängige Vorstellungsmodelle. Einerseits werde Beratung als Informationsvermittlung mithilfe »einer besonderen Gesprächstechnik, die recht leicht, lehr- und lernbar ist « (Engel et al., 2004, S. 33) verstanden. Anderseits suggeriert Beratung eine weniger ausführliche und zeitlich kürzere Form von Therapie (vgl. ebd.). Während das erste Modell weniger hohe Erwartungen an den Beratungsvorgang und dessen Ergebnis knüpft, wird im zweiten deutlich, dass Beratung als etwas Professionelles angesehen wird, dessen Effizienz im Vordergrund steht.

Die Formalisierungsgrade von Beratungen beschreiben einen fließenden Übergang zwischen alltäglichem und professionellem (fachlich spezialisiertem) Handeln. Einrichtungen der Sozialen Arbeit, darunter beispielsweise Beratungsstellen oder Hilfetelefone, sind $\gg$ Arbeitsfelder explizit ausgewiesener Beratung durch Personal mit zumeist professioneller >Beratungsqualifikation « « (Sickendiek et al., 1999, S. 21). Definitorische Ansätze wie dieser reichen jedoch nicht aus, um Reichweite und Intensität diversifizierter Praxisfelder von Beratungen klar zu umreißen. Institutionalisierte Formen sind ebenso wie Gespräche im Alltag dem Bereich der Beratungen zuzuordnen (vgl. ebd.). Anknüpfend daran stelle ich nun die drei maßgeblichen Professionalisierungsgrade von Beratungen vor: nicht-standardisierte, halb-standardisierte und standardisierte Beratung.

Unter nicht-standardisierter Beratung werden alle Formate informeller Beratung gefasst, die außerhalb eines professionell-institutionellen Handlungsrahmens stattfindet (vgl. ebd., S. 22). In Form praktischer oder emotionaler Unterstützung greift sie alltägliche Situationen sozialer Interaktionen auf und beschreibt auf diesem Weg die »Zuwendung zwischen Menschen außerhalb definierter beruflicher Zusammenhänge « (ebd.). Schlussfolgernd daraus kommt ihr als verbindendes Element eine sogenannte Querschnittsfunktion zu, die verschiedene Themen und Tätigkeiten miteinander verbindet (vgl. Nestmann, 2008, S. 74). Beratende Inhalte werden demnach in Settings vermittelt, die auf den ersten Blick nicht als Beratungsgespräche erkannt werden. Beziehungsprobleme, gesundheitliche Sorgen, berufliche oder schulische Schwierigkeiten gelten beispielsweise als klassische Themen von Alltagsberatung. Der Freund_innenkreis, die Familie oder aber auch das Kollegium werden als konkrete Akteur_innen benannt. Verschiedene Anlässe und Motivationen begünstigen das Zustandekommen dieses Beratungsprofils. Dazu zählen vorrangig das Bedürfnis, »sich den Frust von der Seele zu reden « oder das Profitieren von der und die Wertschätzung der Meinung und der Erfahrungen des Gegenübers. Obwohl sie als Formen nicht-professioneller Beratungen gelten, ist wissenschaftlich anerkannt, dass der Großteil der Belastungen allgemeiner Lebensführung und sozialer Konflikte über 
diesen Austausch in Alltagsberatungen bewältigt wird (vgl. Sickendieck et al., 1999, S. 22).

Lassen sich Problemlagen hingegen nicht (ausreichend) im sozialen Nahraum lösen, kann dies dazu führen, dass formelle bzw. halb-standardisierte Beratungsinstanzen aufgesucht werden. Menschen, die sozial isoliert leben, über geringe Gesprächskontakte verfügen oder ihre Problemlage als zu verfahren, zu komplex oder zu massiv einschätzen, um das persönliche Umfeld damit zu konfrontieren, wenden sich an Beratungsstrukturen, die ein höheres Maß an Fachwissen und -kompetenzen aufweisen als die Strukturen der nicht-standardisierten Beratung. Auch Scham und das Bedürfnis nach Schutz der Intimsphäre können als Gründe angeführt werden. Zudem kann es innerhalb des sozialen Bezugsrahmens $\mathrm{zu}$ Situationen kommen, in denen eine sachliche und unbefangene Beratungsperson gewünscht wird. Die Kontaktaufnahme erfolgt entweder, indem jemand eigenständig um Hilfe nachsucht, oder sie wird durch andere initiiert (vgl. ebd.). In diesem Fall ist es jedoch nicht vorrangig wichtig, dass die ausgewählten Beratenden über dezidierte Beratungsqualifikationen verfügen. So fungieren auch Personen als Fachleute, die »aufgrund ihrer Profession, Ausbildung oder sozialen Stellung über ExpertInnenwissen verfügen und zu deren Tätigkeit u.a. Beratung zählt« (Sickendiek et al., 1999, S. 23). Als klassisches Beratungsfeld hierfür gilt die Selbsthilfe. Weiterhin konkretisieren Sickendiek et al., dass » halbformalisierte Beratung als genuiner Anteil unterschiedlicher sozialpädagogischer und psychosozialer Berufe und weiterer sozialer, psychologischer, medizinischer oder juristischer Tätigkeiten, in [denen] die Betreffenden als Professionelle angesprochen sind « (ebd.), anzusehen ist. Dazu zählen zum Beispiel Beratungen im medizinischen Sektor, da Ärzt_innen in der Regel nicht über explizit ausgewiesene Beratungsqualifikationen verfügen.

Für standardisierte Beratungen hingegen ist einerseits eine starke Formalisierung charakteristisch, andererseits sind sie ausdrücklich als professionelle Beratungen in speziellen Einrichtungen ausgewiesen, deren beratendes Expert_innenTeam fundiert ausgebildet ist und über dezidiertes inhaltliches Fachwissen und spezifische methodisch-kommunikative Kompetenzen verfügt (vgl. ebd.).

Im Wesentlichen haben alle Formen von Beratung gemein, dass sie als interaktiver Austausch zwischen mindestens zwei Personen zu verstehen sind, der mithilfe einer praktischen Annäherung das Ziel verfolgt, eine Aufgabe zu lösen bzw. miteinander Lösungsansätze zu erarbeiten (vgl. Zwicker-Pelzer, 2010, S. 13). Beratung orientiert sich laut Zwicker-Pelzer in der Regel an einer akuten Situation, einer aktuellen Entscheidung oder wird als konkrete Problem- bzw. Krisenbewältigung wahrgenommen. Das Beziehungsverhältnis zwischen der be- 
ratenden und der Rat suchenden Person ${ }^{55}$ basiert dabei immer auf Freiwilligkeit (vgl. ebd., S. 21). Die sogenannte »Problemeinsicht « ist der erste Schritt, um Beratung selbstbestimmt in Anspruch zu nehmen. Das impliziert in gleicher Weise, dass der eigene Unterstützungsbedarf erkannt wird und ein generelles Vertrauen in Beratungsangebote besteht. Zwangsberatung ist demnach keine Beratung, da Kontrolle und das schlichte Gewähren von Leistungen in formeller und institutioneller Hinsicht Beratungsgrundsätzen widersprechen und von ihnen getrennt werden müssen (vgl. Sickendiek et al., 1999, S. 224f.).

\section{Der Ansatz psychosozialer Beratung}

Ausgehend von den historischen Entwicklungen und »Anfangsmomenten « von Beratungen Mitte der 1970er Jahre ${ }^{56}$ hat sich die psychosoziale Beratung heute als eigenständige Fachdisziplin etabliert. Resultierend aus der diskursiven Auseinandersetzung der Antipsychiatrie-Bewegung Anfang der 1970er Jahre stehen heutzutage im Hinblick auf psychosoziale Beratung nicht Menschen im Mittelpunkt,

» die Heilung bei Krankheit oder psychischer Störung [suchen], sondern Menschen in verschiedensten, meist belastenden Lebenskonstellationen, die bei unterschiedlichen Anforderungen, Problemen und Krisen professionelle Hilfe bei der Orientierung, bei der Entscheidung und Planung von Veränderung, bei der Wahl ihrer Ziele und Problemlösungen sowie beim Einschlagen, Gehen und Überprüfen der veränderten, neuen Wege suchen. Diese Herkunft prägt noch heute die Identität psychosozialer Beratung « (Nestmann, 2008, S. 73).

Die Neuausrichtungen infolge des inhaltlichen Paradigmenwechsels gehen nicht zuletzt auf die Initiative engagierter Selbsthilfeverbände zurück, deren Einflussnahme das allgemeine Ansehen sozialer Dienste aufwertete und zugleich

55 Auf die Bezeichnung »Klient_in« wird verzichtet, da diese Begrifflichkeit professionelle Devianz charakterisiert (vgl. Zwicker-Pelzer, 2010, S. 24) und somit als stigmatisierend wahrgenommen werden könnte. Im Hinblick auf eine herrschaftskritische Analyse impliziert die Bezeichnung Klient_in nach Tuggener eine Subjekt-Objekt-Beziehung anstelle einer anzustrebenden Subjekt-Subjekt-Interaktion auf gleicher Ebene (vgl. Tuggener, 1983, zit. n. Zwicker-Pelzer, 2010, S. 24). Letztendlich ist ein kritisch-reflexiver Umgang anzustreben.

56 Die 1970er Jahre gelten als Dekade der Herausbildung von Beratungen und beschreiben einen regelrechten Entwicklungsboom, indem zahlreiche Publikationen zum Thema erschienen und die ersten Versuche unternommen wurden, Beratung als Element von Ausbildung und Forschung zu verankern (vgl. Nestmann, 2008, S. 72). 
wesentlich zur Entwicklung von Beratungen und ihrer sozialpädagogischen Kernstrukturen beitrug. Die wohl bekanntesten Beratungsprojekte jener Zeit sind u. a. im Bereich der autonomen Frauenhäuser sowie der feministischen Frauengesundheitszentren bis heute existent (vgl. ebd., S. 72).

Psychosoziale Beratung leistet einen wichtigen Beitrag zur Professionalisierung sozialer Arbeit. Ihr wesentlicher Grundzug lässt sich wie folgt kennzeichnen: eine unabhängige Expertise, die durch eine freiwillige Inanspruchnahme der Unterstützung, durch individuelle Selbstbestimmung und in Form niedrigschwelliger Einzel- und Gruppenangebote Impulse von sozialpädagogischer und psychologischer Handlungsorientierung miteinander verbindet. Bis dato waren beratende Tätigkeiten auf den Bereich der klinisch-psychologischen Diagnostik beschränkt und durch Stigmatisierung, Kontrolle, Zwang und Dirigismus gekennzeichnet (vgl. ebd., S. 73f.). Als Kennzeichen gegenwärtiger psychosozialer Beratungen werden an dieser Stelle vier Identitätspfeiler vorgestellt, die die zentralen Funktionen in beratenden Unterstützungsprozessen darstellen: Informationsbalance und Entscheidungsmanagement, Prävention und Gesundheitsförderung, Bewältigungshilfe sowie Entwicklungsförderung und Lebenslaufbegleitung (vgl. ebd., S. 78).

\section{Informationsbalance und Entscheidungsmanagement}

Der Begriff »Informationsbalance « geht auf Frank Engel zurück und beschreibt, dass Beratungen seit dem Aufkommen neuer Kommunikationsmedien über eine bloße Weitergabe von Informationen hinausgehen und ein ausgewogenes Verhältnis zwischen informativen und beratenden Inhalten bieten müssen (vgl. Engel, 2002, zit. n. Nestmann, 2008, S. 79). Zwar sei es weiterhin Aufgabe von Beratung, Informationslücken zu schließen, doch sehen sich die Beratungsinstanzen derzeit mit einer Fülle an ungefilterten Überinformationen konfrontiert, die nicht zwingend zu einer besseren Informiertheit der Beratenen führt. Entscheidungsschwierigkeiten der betreffenden Personen werden dadurch nicht unbedingt gelöst (vgl. Nestmann, 2008, S. 79). Fachwissen muss demnach vermittelbar gestaltet sein und kompetent umgesetzt werden. So spricht Nestmann davon, dass »neben der professionellen psychosozialen Beratung meist auch informelle Einflussnahme erfolgt« (ebd., S. 80), zum Beispiel durch Alltagsgespräche oder selbstständiges Informieren (vgl. ebd.). Diese Tatsache tritt auch im Bereich von Inter*-Beratung zutage. Nicht selten passiert es, dass Inters* besser informiert sind als Ärzt_innen und/oder Berater_innen. Dies ist vor allem darauf zurück zu 
führen, dass reguläre Praxen und Beratungsstellen nicht in jedem Bereich über Spezialwissen verfügen (können), was wiederum zu der Annahme führt, dass im Kontext von Intergeschlechtlichkeit die Implementierung spezialisierter Fachberatungsstellen sinnvoll erscheint.

Die Bereitschaft ratsuchender Personen, sich innerhalb von Beratungssituationen zu öffnen, erfordert ein aktives Vertrauen zwischen Beratungsinstanzen und Ratsuchenden. Vertrauensaufbau auf der einen, Begrenztheit dauerhaft gesicherter Expert_innenaussagen auf der anderen Seite stellen die Beratung vor eine große Herausforderung. Was heute noch als gesicherte wissenschaftliche Aussage gilt, kann morgen schon widerlegt sein. Es gilt, sowohl als Beratende_r, als auch als Beratene_r flexibel zu bleiben und zu erkennen, dass auch Beratung gesellschaftlichen Veränderungen und realen Paradoxien, Unsicherheits- und Ambiguitätsfaktoren unterliegt (vgl. ebd.). Dies erfordert seitens der Beratenen die Einsicht einer » positiven Nichtsicherheit «. Dieses auf H. B. Gelatt zurückgehende Konzept umschreibt im Wesentlichen, dass sich Meinungen und Ansichten ändern können, ohne dass daraus zwangsläufig oder in einem kausalen Zusammenhang Unsicherheiten entstehen müssen. Vielmehr gehe es darum, »Ambiguität sowie Inkonsistenz akzeptieren zu können und Intuition ernst zu nehmen « (ebd., S. 81). Ferner bedeutet dies, ein subjektives Körpergefühl zu entwickeln, um sensibel auf das eigene »Bauchgefühl « zu reagieren. Es geht dabei nicht mehr nur um wissenschaftlich gesicherte Aussagen, die als unumstößliche faktische Wahrheit gesehen werden; vielmehr gewinnen auch die »Intelligenz des Unbewussten « wie auch die »Macht der Intuition « wissenschaftlich an Ansehen (vgl. ebd., S. 82). Dieser neue Blick, der sich auf das positive Zutrauen in eigene Ansichten und Einschätzungen richtet, führt zu einem Wandel der Rolle der Ratsuchenden. Sie verlieren dementsprechend den Status ihrer Passivität, werden selbst aktiv und in den Beratungsverlauf mit einbezogen. Sie konsumieren nicht mehr nur Fachwissen, sondern gestalten den Prozess selbstbestimmt, nach eigenem Ermessen und mit dem Fokus auf eigene Bedürfnisse, mit. So heißt es bei Nestmann:

$\gg$ Entscheidungsprozesse werden immer mehr zu $>$ Entdeckungs- $<$ Prozessen. Ziele zu entdecken, auch andere und neue, statt nur ein Ziel erreichen zu wollen, wird wichtig: eine Abkehr von einem radikalen >Sei realistisch< hin zu einem >Nicht sicher - aber Für-möglich-halten<. Wichtig ist, Anregung für neue Bedürfnisse, Wünsche und Ziele zu bekommen. Immer nur klare Ziele können eingrenzen und halten oft von neuen Möglichkeiten ab. Beraten heißt somit auch helfen, Subjektivität zu entwickeln und Überzeugungen aufgeben zu können oder Entscheidungen auf die Probe zu stellen « (Nestmann, 2008, S. 81). 


\section{Prävention und Gesundheitsförderung}

Anspruch emanzipatorischer Beratungsansätze ist es, Beratung nicht mehr im Schatten eines medizinischen Modells von Behandlung und Versorgung zu verorten (vgl. Sickendiek et al., 1999, S. 222). Insofern setzt Prävention da an, wo der » fatalen Logik des ausschließlichen Reagierens, kurativen Hinterherhinkens und Wiederherstellens von Normalität und Funktionsfähigkeit « (Nestmann, 2008, S. 82) Einhalt geboten wird, neue Denkrichtungen entwickelt und Schwerpunkte gesetzt werden (vgl. ebd.). Die allgemeine Tendenz, Beratungskontakte erst im Falle länger andauernder und verschärfter Problemlagen aufzunehmen, zeigt nicht selten bereits ernste und irreparable Belastungsfolgen auf (vgl. Sickendiek et al., 1999, S. 222). Je mehr sich Beratung als präventiv orientierte Profession versteht, desto größer ist die Wahrscheinlichkeit, Menschen bereits im Vorfeld der Problementstehung zu erreichen (vgl. ebd.). Präventive Ansätze haben das Potenzial, zukünftige Risiken vorwegzunehmen, Chancen frühzeitig abzuwägen und mögliche Handlungskonsequenzen zu reflektieren (vgl. Nestmann, 2008, S. 82). Somit wirkt Beratung im Vorfeld bereits nachhaltig. Allerdings ist bei ebendiesen Aussagen und Grundhaltungen darauf zu achten, dass dies nicht in gleichem Maß für den Bereich präventiver Diagnostik gilt. In der Vergangenheit wurde nicht selten unter Zuhilfenahme von Wahrscheinlichkeiten präventiv in Behandlungsprozessen argumentiert, zum Beispiel in Bezug auf mögliche Tumorrisiken als Begründung für eine empfohlene Gonadektomie. Konkret bezieht sich präventives Handeln auf die Förderung und Erleichterung der Lebensführung durch das konkrete »Stärken von individuellen Kompetenzen und Selbsthilferessourcen zur Erreichung von Handlungsfähigkeit und Lebenszufriedenheit, [...] eingebunden in die Förderung von stützenden und hilfreichen Gemeinschaften und in das Gestalten sozial- und gesundheitsförderlicher Lebensverhältnisse « (Nestmann 2002, zit. n. Nestmann, 2008, S. 82f.). Dabei kommt der Orientierung an individueller Lebenswelt und Alltag ${ }^{57}$ eine besondere Bedeutung zu. Auch hier ist es ratsam, zu beachten, dass durch einseitige Argumentationen » für Prävention « Personen-

57 Alltags- und Lebensweltorientierung geht auf das Konzept von Hans Thiersch zurück und beeinflusste maßgeblich die Entwicklung von Beratungen (vgl. Nestmann, 2008, S. 75). Lebenswelt und Alltag als zentrale Ansatz- und Bezugspunkte von psychosozialer Beratung greifen konkrete Beratungsaufträge da auf, wo sie entstehen. Mit Blick auf eine mögliche Ursachenanalyse im sozialen, ökonomischen und ökologischen Umfeld erarbeiten sie gemeinsam-partizipativ und in akzeptierender sowie solidarischer Grundhaltung Möglichkeiten, die Rat suchende Person zu unterstützen, selbst aktiv zu werden und dabei ihre Lebenssituation zu erleichtern (vgl. ebd.). 
kreise, die nicht alle Risiken im Vorfeld bedenken wollen oder können - indem sie zum Beispiel »Bare Backing ${ }^{58}$ praktizieren oder, im Fall von Schwangerschaft, auf prädiktive Diagnostik verzichten -, zunehmend stigmatisiert werden.

Im Kontext von Beratung geht es darum, »bisher unreflektierte und unverrückbare Positionen [zu] hinterfragen, neue Perspektiven und Handlungsformen [zu] ermöglichen, alltägliche Einschränkungen und Routinen in ihren Ursachen [zu] klären und neue Entwicklungsräume [zu] öffnen sowie Gelegenheit zum Üben von neuem Denken, Fühlen und Handeln [zu] schaffen « (Nestmann, 2008, S. 83). Die Gesamtansicht des Lebenskontextes von Personen ist wichtig, um mögliche Einschränkungen zu eruieren und präventive Handlungsmöglichkeiten zu gewinnen (vgl. Sickendiek et al., 1999, S. 222). Allerdings ist dabei immer der Aspekt der Freiwilligkeit mitzudenken. Entscheidungen gegen ein mögliches Vorgehen müssen von der Beratungsperson gegen jede vermeintliche Sinnhaftigkeit akzeptiert und mitgetragen werden. Daraus resultiert die Einsicht, dass es nie nur ein einziger Grund ist, der zu belastenden Situationen führt. In der Regel ergeben sich Schwierigkeiten aus einem Komplex unterschiedlicher Zusammenhänge, die auf mehreren Ebenen ihren Ursprung haben und in der Äußerung einer mehr oder weniger konkreten Problemstellung zusammenkommen. Genau deshalb ist es wichtig, nicht nur die Ursachengenese in den Blick zu nehmen. Soziale, ökonomische, ökologische und kulturelle Bedingungen müssen zusammen analysiert werden (vgl. ebd.).

Insgesamt hat sich Beratung in Richtung basaler Bedürfnisse und Forderungen geöffnet und sieht darin die Möglichkeit für beratene Personen, selbstaktiv zu handeln. Demnach ist es von besonderer Wichtigkeit, dass psychosoziale Beratung in Lebensweltkontexte integriert wird. Konsequenterweise muss sie demnach auch vor Ort angeboten werden. Praktisch bedeutet das, die herkömmliche »Komm-Struktur « sozialpädagogischer Beratungsstellen zu verlassen, um aufsuchende und niedrigschwellige Sozialarbeit bzw. mobile Beratung als anzustrebendes Beratungsprofil anzubieten. Orte, Räume und zeitliche Strukturen von klassischen Beratungsbedingungen müssen ihre starre Rahmung zugunsten einer höheren Flexibilität aufgeben. Besonders bedeutsam werden zukünftig virtuelle Settings. Nestmann empfiehlt diesbezüglich, die klassische Face-to-Face-Beratung und Online-Angebote im Wechsel bzw. gleichermaßen anzubieten. Dieses methodische Vorgehen ist in der Wissenschaft unter der Bezeichnung »Blended Counseling « bekannt (vgl. Nestmann, 2008, S. 84). Soziale Beziehungen

58 »Bare Backing« bezeichnet die bewusste Entscheidung des ungeschützten Geschlechtsverkehrs (unter Umständen an unsicheren Orten) unter Männern. 
gelten zwar einerseits als mögliche Ursachenquellen von Stress und Problemen, sind andererseits aber auch eine wertvolle Stütze bei der Problembewältigung oder aber auch bereits im Vorfeld bei der Vermeidung von und dem Schutz vor schwierigen Lebenslagen. Ein Netz sich gegenseitig unterstützender Vertrauter (Familie, Freund_innenkreis, Partner_innen, Nachbar_innen, Kollegium) oder Peers übernimmt die Funktion der » wichtigsten präventiven wie belastungs- und stressabpuffernden Potenziale unseres Lebens « (ebd.). Im Sinne einer präventiven und gesundheitsfördernden Beratung kann nicht nur der Mensch isoliert betrachtet werden. Vielmehr müssen auch die jeweiligen Lebensumstände und äußeren Faktoren berücksichtigt werden, denn:

»Beziehungen können Sicherheit, Einbettung, soziale Anerkennung, Würdigung des Einzelnen bieten, sie tragen zur Lebensführung bei und verhindern krankmachende Isolation und Einsamkeit. Sie haben das Potenzial der frühen Wahrnehmung von Risiken [...] und beeinflussen schließlich unseren Zugang zur professionellen Beratung « (ebd.).

Aus den im Vorfeld genannten Gründen lässt sich die Wichtigkeit von Peer-Support und Peer-Beratung ableiten. Für die sozialpädagogische und psychosoziale Arbeit mit Inters* sind sie demnach unerlässlich.

\section{Bewältigungshilfe}

Als wesentliche Hilfe zur Problembewältigung wird das Beratungsgespräch angesehen. Meist in einer Eins-zu-eins-Kombination (zum Beispiel » face to face « oder im virtuellen Raum), ermöglicht diese Beratungssituation, Kontakt aufzunehmen und im Gegenüber auf jemanden zu treffen, der_die aktiv zuhört. Die Kommunikation innerhalb dieser geschützten Struktur hat das Potenzial parallel auf den Alltag übertragen zu werden. In der Regel ist es so, dass sich in Beratungssituationen der gesellschaftliche Alltag in Form von Rollen und Verhaltensmustern widerspiegelt. Das heißt, Probleme, die im Alltag auftauchen, werden auch innerhalb beratender Strukturen sichtbar und umgekehrt. Ebenso heißt dies aber auch: Verlaufen Gesprächsaufnahmen in der Beratung gut und zufriedenstellend, kann dies auch in der Übertragung auf den Alltag glücken (vgl. Nestmann, 2008, S. 86). Beratungen bieten in ihrer Rolle der Bewältigungshilfe an, die eigene Identität (wieder) zu finden und zu sichern und vermitteln die Einsicht, aktiv das eigene Leben mitgestalten zu können. Beratungen in diesem Sinn schaffen Raum, um 
neue Selbstentwürfe auszuprobieren (vgl. ebd.), sie befähigen zum selbstaktiven Handeln. Somit übernimmt Beratung eine Schwellenfunktion, die als Weg zurück zu gesellschaftlicher Teilhabe und Verhandlung persönlicher Angelegenheiten zu verstehen ist (vgl. ebd.). Grundsätzlich beschreibt dieser Identitätspfeiler den Prozess der Reintegration in den Lebensalltag durch das Wiedererlangen eigener Handlungsfähigkeiten. In Abkehr von rein problemfixierten Beratungs- und Therapieansichten entspricht diese Haltung einem ressourcenorientierten und -fördernden Ansatz, der den Kerngedanken psychosozialer Beratung erfasst (vgl. ebd.). Im Mittelpunkt des Interesses steht das Ansetzen an persönlichen Stärken und interpersonalen Potenzialen der Ratsuchenden bei gleichzeitiger Orientierung an ihrem sozialen Netz, um diesen Prozess anhand eines systemischen Ansatzes zu unterstützen, indem Kontext- und Umweltressourcen einbezogen werden (vgl. ebd., S. 88). Insbesondere geht es dabei um die Stärkung des Selbstwert- und Kohärenzgefühls ${ }^{59}$, was sich in positiver Weise auf Wohlbefinden und Gesundheit auswirkt und darüber wiederum durch Zutrauen in die eigenen Fähigkeiten zum Bewältigungserfolg führen kann. Es stellt also im Resultat dar, was allgemein unter dem Begriff »Hilfe zur Selbsthilfe « zu verstehen ist. Damit eng verknüpft, zeigt sich auch in diesem Fall wieder der Einfluss ökonomischer und ökologischer Faktoren, zum Beispiel durch Schule, Beruf und/oder kulturelle Einbettung. Im Gegensatz zu anderen Beratungsansätzen werden hier »Betroffene « selbst als die markanten sozialen Akteur_innen angesehen - als Expert_innen in eigener Sache. In einer gemeinnützigen Weise stellen sie wiederum ihre Ressourcen ihrer sozialen Umgebung zur Verfügung. Sie integrieren die Hilfeleistung in weitere soziale Bezugssysteme, formieren und unterstützen Prozesse der Selbstorganisierung von Peers und verknüpfen formelle mit alltäglichen Hilfequellen. Die Organisationsformen des »Peer-Supports « haben großen Einfluss und Auswirkungen auf die Wiedererlangung und die Stabilisierung persönlicher Lebensbewältigungsstrategien und nehmen dementsprechend einen überaus wichtigen Stellenwert hinsichtlich psychosozialer Beratung im Allgemeinen und einer gerechten Inter*Beratung im Speziellen ein (vgl. ebd.).

Im Hinblick auf gesellschaftliche Veränderungen und eine historisch etablierte Pluralisierung von Lebensentwürfen und Begehrensformen müssen sich Beratungsstile neuen und vielfältigen Nutzer_innengruppen öffnen. Die Akzep-

59 Damit ist nicht eine Kohärenz im Sinne Butler'scher Geschlechtertheorie gemeint (siehe Butler, 1991, S. 38); vielmehr geht der Ausdruck auf das Konzept der Salutogenese nach Aaron Antonovsky (1997) zurück. In diesem Ansatz wird Gesundheit nicht mehr nur als Abwesenheit von Krankheit charakterisiert, sondern bezieht soziale und psychische Dimension ein (vgl. Antonovsky, 1997, S. 29ff.). 
tanz und der Einbezug von Diversität und inklusiven Bestrebungen kommen vor allem den Gruppen zugute, die bislang übersehen oder bewusst ausgeschlossen wurden (vgl. ebd.). Das stellt insbesondere für Inters* eine Chance und Möglichkeit dar, wahrgenommen zu werden und ihre Bedürfnisse, Rechte und Forderungen adäquat geltend zu machen. Für die Beratungspraxis heißt das, $\mathrm{Zu}$ gänge zu Beratungen, $\mathrm{Ab}$ - und Verläufe von Beratungsprozessen sowie Arten und Formen der Unterstützungsleistungen divers zu gestalten (vgl. ebd., S. 89). In Fachkreisen als »Counseling Diversity « bezeichnet, werden neue und offene Konzepte uniformen Programmen gegenübergestellt. Ausgehend von dieser neuen Denkrichtung fordern psychosoziale Beratungen Toleranz und den Erwerb von Kompetenzen im Umgang mit erweiterter Normalität (vgl. ebd.). Sie verstehen sich als Interventionsformen gegen (Hetero-)Normativität und beziehen explizit intersektionale ${ }^{60}$ Aspekte und Zusammenhänge, zum Beispiel Gender und Migration, einschließlich der kritischen Analyse der zugrunde liegenden Herrschaftsverhältnisse, in ihre (Beratungs-)Konzepte ein. Der Reflexion der eigenen Rolle als Berater_in kommt dementsprechend eine wichtige Aufgabe zu. Durch das Realisieren eigener struktureller Vorteile wird die gesamte Beratungsbeziehung neu kontextualisiert und in Szene gesetzt. Empathie und Sensibilität verlieren ihren paternalistischen Anspruch und erfahren völlig neue Bedeutungen, da sie erstmalig mit eigenen Handlungen und Mechanismen kritisch in Verbindung gebracht werden. Ebenso tragen Reflexionsprozesse dazu bei, sich mit dem Gegenüber, der Struktur und dem, was man bereits als Ansatz im Kopf für richtig erachtet, auseinanderzusetzen - insofern, als Unterschiede nicht mehr automatisch in Machtdifferenzen und Ungleichheiten münden. Sobald »Machtungleichheiten bestehen, können Stereotype auch in der Beratung zur Verstärkung von Marginalisierung und Ausschluss der ohnmächtigeren Gruppe beitragen « (Nestmann, 2008, S. 90). Um diese Ansätze auch in den Bereich des Methodenpools zu integrieren, müssen neue, vielfältigere Ideen folgende Kriterien erfüllen bzw. bestehende Methoden ihnen angeglichen, überarbeitet oder erweitert werden. Als inklusives Kriterium gilt es, wenn Methoden »reflexiv, aufklärend, unterstützend, konfrontativ, ressourcenfördernd, übend, kathartisch

60 Das komplexe Ineinandergreifen unterschiedlicher machtvoller Kategorien wird unter wissenschaftlicher Betrachtung als Intersektionalität bezeichnet (vgl. Smykalla \& Vinz, 2011, S. 9). Hierarchische Differenzlinien (Geschlecht, Sexualität, Klasse, Alter, Ability, Herkunft) bilden die Grundlage moderner Gesellschaftsorganisationen. Differenzen sind dabei als Resultat sozialer Konstruktionen zu begreifen und verstärken sich gegenseitig (vgl. Tuider, 2008, S. 256). Sie beschreiben keine inhaltliche, sondern eine strukturelle Art des Unterschieds (vgl. Smykalla \& Vinz, 2011, S. 232). Ihre Verschränkungspunkte bilden sogenannte »inter-sections«. 
und katalytisch [sind] [...], vor allem auch dann, wenn psychosoziale Beratung über Individuen hinaus sich auf soziale Aggregate richtet « (ebd.).

\section{Entwicklungsförderung und Lebenslaufbegleitung}

Im Zuge von präventiven Maßnahmen und Ressourcenförderung übernimmt die Entwicklungsförderung eine Unterstützungsaufgabe, die Anreize schafft und Schutzräume errichtet, um ein eigenes Selbstbild, die eigene Identität zu entfalten. Entwicklungsförderung bezeichnet den Interaktionismus zwischen Personen und ihrem Umfeld und bezieht immer auch zugleich Ressourcen größerer, überindividueller Systeme wie Familien, Gruppen oder Organisationen ein. Dabei geht es explizit darum, Möglichkeiten zu schaffen, in soziale Interaktion zu treten, um sich auszuprobieren und sich unabhängig von gesellschaftlichen Normierungen zu entdecken (vgl. Nestmann, 2008, S. 91). Im Kontext von Intergeschlechtlichkeit bedeutet dies die Förderung der Entwicklung eines stabilen Selbstwertes und die Negierung und Verweigerung eines lebensbegleitenden Schweigegebots innerhalb der eigenen sozialen Familiensysteme und Freund_innenkreise. Es bedeutet ein Aufwachsen in einer offenen Geschlechtsidentität, ohne Zwang zur Heteronormativität. Es bedeutet für Eltern unter Umständen, Kompromisse schließen zu müssen, um ihren Kindern ein Aufwachsen in einer nicht bipolaren Geschlechtsidentität ohne Scham, ohne Verbote und ohne Rechtfertigungsdruck gegenüber Dritten zu ermöglichen. Psychosoziale Beratung bietet dabei Orientierung, fördert Entscheidungen und unterstützt die Beratenen in ihrem Handeln (vgl. ebd., S. 92f.). Sie übernimmt die Aufgabe individueller und alltäglicher Selbsthilfeförderung. Zentrale Merkmale sind Akzeptanz, Bedürfnisorientierung und Selbstbestimmung. Erst durch die Aktivierung von Selbstbestimmungsbestrebungen werden Strategien sozialer Teilhabe nutzbar. Eigene Bedürfnisse und Rechte werden erkannt und eingefordert. Diese Selbstermächtigung durch Empowerment bringt die Menschen dazu, Einfluss auf und Kontrolle über das eigene Leben wiederzuerlangen. Mit steigender Selbstständigkeit der Beratenen übernimmt Beratung die Funktion der Prozessbegleitung. Durch das Üben neuer Denk- und Handlungsmuster und das Ausprobieren dieser verschiedenen Optionen in realen Zusammenhängen erwerben die Beratenen eine Fülle an Kompetenzen. Jedoch verlaufen Beratungsprozesse nicht immer kongruent und somit ist es wichtig, in akuten Situationen erneut Anbindung an Beratungsinstanzen zu finden (vgl. ebd.). Eine Gewährleistung dessen unterstreicht den dynamischen Charakter psychosozialer Beratungen. 


\section{Methoden psychosozialer Inter*-Beratung}

Beratungen übernehmen nicht nur im psychosozialen Bereich eine wichtige Funktion, sondern auch im Hinblick auf Gesundheitsförderung, im Bildungs- und beruflichen Sektor, in der Erziehung und im Kontext gruppenspezifischer Anforderungen (Alter, sexuelle Orientierung, Eltern). Eine inter*-spezifische Beratung braucht soziale und psychosoziale Impulse, die durch unterschiedliche Disziplinen vorangetrieben werden. Es erscheint daher zukünftig unerlässlich, eine eigenständige professionelle Beratungsidentität für den Bereich Intergeschlechtlichkeit zu entwickeln und zu verankern. Eine interdisziplinäre multidimensionale Inter*-Beratung muss auf vier Ebenen umgesetzt werden: theoretisch-konzeptionell, praktisch-methodisch, berufspolitisch-institutionell sowie in Wissenschaft und Forschung (vgl. Nestmann, 2008, S. 92f.). Soziale Konstellationen und methodische Dimensionen des Beratungshandelns sind vielfältig und abhängig von der jeweiligen Ausgangssituation. Grundsätzlich ist eine reflektierende Handlungspraxis zum Erschließen diversifizierter Unterstützungsquellen maßgeblich.

Innerhalb der Beratungsverläufe stehen die Methoden im Mittelpunkt der Aufmerksamkeit. Nestmann beschreibt Methoden als $\gg$ Nahtstelle zwischen Erkenntnis des Problems, Handlungskonsequenzen und Zieldefinition « und bezeichnet sie als »Problembearbeitung im Felde der Problementstehung « (ebd., S. 75). Abgeleitet aus klient_innenzentrierten und psychotherapeutischen Konzepten von Beratung vereinen diese Arbeitsansätze Kommunikations- und Gesprächsführungstechniken grundlegender Beratungsstrategien, darunter sowohl Methoden des aktiven Zuhörens, des Feedbacks und des Paraphrasierens ${ }^{61}$ als auch das Verbalisieren und Fokussieren von Emotionen (vgl. Sickendiek et al., 1999, S. 133). Diesem Verständnis zufolge handelt es sich bei einer Methode um »eine bewusst und geplant eingesetzte, häufig bereits erprobte Handlungsweise, mit der ein bestimmtes Ziel erreicht werden soll « (ebd.). Eine bloße Reduktion von Beratungen auf Techniken der Gesprächsführung wäre hingegen verkürzt. Ziel methodischen Handelns ist ebenso die Erprobung neuer Lernerfahrungen, die Lernen am Modell ermöglicht und nicht auf die Tätigkeit professionell ausgebildeter Berater_innen begrenzt ist (vgl. ebd.).

Auch die Organisierung in Selbsthilfegruppen in Form des Peer-Counseling trägt wesentlich dazu bei, neue Identifikationsmöglichkeiten zu erschließen.

61 Paraphrasieren ist eine Technik der Gesprächsführung, um etwas sinngemäß in eigenen Worten zusammenzufassen, wiederzugeben und um zu überprüfen, ob das Gesagte so verstanden wurde, wie es intendiert war. 
Ausgehend von entwicklungs-, sozial- und gesundheitspsychologischen Erklärungsmodellen sind diese neueren Formen der Interaktion ursprünglich vor dem Hintergrund der HIV/Aids-Prävention und der Gesundheitsförderung entstanden und werden zur Stabilisierung von Peers eingesetzt (vgl. Backes, 2004, S. 19f.). Peer-Counseling kann sich auf unterschiedliche Bereiche und Themen beziehen, vereint jedoch grundsätzlich Menschen, die gleiche Lebensumstände und Erfahrungen teilen, zum Beispiel durch einen ähnlichen soziokulturellen Hintergrund oder das Erleben bestimmter Ausgrenzungserfahrungen (vgl. Berliner Zentrum für Selbstbestimmtes Leben behinderter Menschen e. V., 2014). Durch das Wissen um gleiche Erfahrungen und Ausgangssituationen, die Fähigkeit, sich in die Lage des Gegenübers hineinzuversetzen und mitzufühlen, entwickelt sich eine gemeinsame Vertrauensbasis (vgl. ebd.). Peer-Ansätzen wird »das Potential zugeschrieben, Randgruppen anzusprechen, die durch andere Präventionsstrategien nur schlecht zu erreichen sind « (Appel, 2002, S. 22). Sie dienen demnach insbesondere sowohl der Stressbewältigung als auch der »Stärkung individueller Widerstandskompetenzen gegen negativen Druck « (Backes, 2004, S. 19). Im Rahmen der Selbsthilfe wird ersichtlich, dass Peer-basierte Arbeitsansätze stark aktionsorientiert sind und auf Netzwerkförderung und Empowerment abzielen (vgl. ebd., S. 19f.). Sie ermöglichen anderen »Betroffenen «, ein höheres Maß an Selbstbestimmung über ihre aktuelle Lebenssituation zu erreichen (vgl. Appel, 2002, S. 21).

Nicht immer verfügen Peer-Counselors über dezidierte Beratungsqualifikationen. Umso wichtiger ist es auch hier, sich gegenüber einer potenziellen Überforderung durch die gravierenden Probleme der Ratsuchenden zu schützen. Wichtig für Multiplikator_innen ist es, die eigenen Grenzen zu kennen und zu wahren (vgl. ebd., S. 20). Ein professioneller Umgang wäre, entweder Weitervermittlungskompetenzen zu erwerben oder eine Kooperation mit standardisierten Beratungsinstitutionen einzugehen. Der letztgenannte Punkt spricht eindeutig dafür, professionelle inter*-spezifische Beratungsangebote, die in enger Zusammenarbeit mit den Selbsthilfeorganisationen stehen, zu implementieren. Prinzipiell gilt es sowohl für Peer-basierte als auch für andere Formen der inter*-spezifischen Beratung, sich an folgenden Kriterien zu orientieren (vgl. Berliner Zentrum für Selbstbestimmtes Leben behinderter Menschen e. V., 2014):

$>$ Ressourcen- anstelle von Problem- und Defizitorientierung

$>$ Bejahung statt Verdrängung der eigenen Intergeschlechtlichkeit

$>$ Förderung eines den eigenen Bedürfnissen gerechten Umgangs mit der geschlechtlichen Varianz 
$>$ Erkennen und Abkehr von zugeschriebenen Rollen (Marginalisierung ${ }^{62}$ und (Selbst-)Viktimisierung) und Abhängigkeiten (Paternalismus ${ }^{63}$, medizinische Autoritäten)

$>$ Entwicklung und Förderung von Selbstermächtigungsstrategien (Peer-Support) und Organisation von Widerstand

$>$ Achtung der eigenen und anderer Persönlichkeiten durch Egalität, Akzeptanz, Wertschätzung und Einfühlungsvermögen

$>$ nicht-direktive Gesprächsführung und Krisenbewältigung (z. B. klient_innenzentrierte und systemische Ansätze)

$>$ Prozesshaftigkeit gewährleisten und Begleitung außerhalb beratender Strukturen anbieten (z. B. zur Agentur für Arbeit bzw. zum Jobcenter), Unterstützung bei der Suche nach qualifiziertem medizinischem Personal (z. B. zu Endokrinolg_innen, Therapeut_innen, zur Akteneinsicht in medizinischen Kliniken etc.), Strategien zur Problemlösung gemeinsam entwickeln (z.B. Methodik der persönlichen Zukunftsplanung und partizipativen Qualitätsentwicklung)

Um diversen Lebensumständen, Persönlichkeiten und Kontexten gerecht zu werden, bedarf es einer spezifizierten Differenzierung der Methodenvielfalt (vgl. Sickendiek et al., 1999, S. 134). Als Orientierung dient die Vorstellung, dass sich aus einer konkreten Problemlage ein klar definiertes Beratungsziel entwickeln und somit ein interaktiver Beratungsprozess initiieren lässt. Methoden gelten dabei als wesentliche Grundlage des Beratungs- und Beziehungshandelns. Abhängig von Person und Anliegen, dem anvisierten Ziel, der Beratungskonzeption, -rolle und -funktion, dem Setting und der jeweiligen Phase im Beratungsprozess ist Beratung multimethodisch gefordert und muss die geeigneten Methoden ziel- und personenspezifisch auswählen und kombinieren (vgl. ebd., S. 135). Es ist ratsam, unterschiedliche Disziplinen mitzudenken:

»[Ich] halte das für sehr sinnvoll, dass das Menschen aus der Sexualpädagogik machen, [...] ich glaube, das ist schon wichtig, weil die auch noch mal andere Methoden haben. [...] [Die] ganze Geschichte kann man ja auch ein bisschen spielerischer angehen. [...] Dadurch nimmt man ihm ja so diese [...] klinische Schwere. Und darin

62 Marginalisierung ist ein Prozess, der Abweichungen bzw. Akteur_innen eines von dem der hegemonialen Gesellschaftsnorm abweichenden Verhaltens ausschließt. Umgangssprachlich ist die Rede von Randgruppen.

63 Paternalismus suggeriert affirmative Fürsorge und Wohlwollen, ist aber letztendlich eine Form der Bevormundung. 
sehe ich schon eine Chance. Ich glaube auch, dass,[...] wenn wir dazu kommen, dass wir $[\ldots]$ zu einem generellen Beratungsangebot [...] für intersexuelle Kinder und ihre Familien kommen, [...] dann werden [...] diese Beratungsgespräche auch nicht mehr so schwer sein. Das, was wir bisher immer [hatten], das war immer OpferBeratung «(Veith, 2014, S. 26).

Anforderungen und Belastungen sind häufig sehr komplex und treffen auf heterogene Konstellationen von Personen und Problemen (vgl. Sickendiek et al., 1999, S. 136). Aus diesem Grund wird das Methodenspektrum »gerade in psychosozialen und sozialpädagogischen Beratungszusammenhängen erweitert, wo multidimensionale Problemstellungen auch multidimensionale Beratungsanstrengungen erfordern « (ebd., S. 135). Somit ist Beratung nicht nur multimethodisch angelegt, sondern auch »eklektisch-integrativ « orientiert. Eklektische Methoden bedienen sich unterschiedlicher Stile und setzen die Elemente gemäß der konkreten Anforderung der Fallkonstellationen systematisch neu zusammen, bei gleichzeitiger Abkehr vom klassisch festgelegten Methodenkanon (vgl. ebd., S. 137). Das Streben nach immer effektiveren und passenderen Hilfen erfordert eine vielfältige Bandbreite beraterischer und therapeutischer Techniken und Kompetenzen (vgl. ebd., S. 136f.). Untersuchungen der »Common-Factor-Forschung ${ }^{64}$ resultieren in der Annahme, dass Wirkungsweisen und Effektivität des gewählten Methodenpools neben ihren Inhalten auch in ihren Funktionen begründet liegen. Demzufolge bieten eklektische Techniken Aussicht auf neue Lernerfahrungen, steigern das Vertrauen in Besserung, räumen Fortschritte und Erfolgserlebnisse ein, agieren im sozialen Kontext wider demoralisierende Entfremdung und bieten emotionale Anregungen (vgl. ebd., S. 137ff.).

Die elaboriertesten Ergebnisse dieser Analysen weist die Forscher_innengruppe um Klaus Grawe vor. Das erste der vier zentralen Prinzipien beschreibt die »aktive Hilfe zur Problembewältigung « mit dem Leitsatz: »Vom Nicht-andersKönnen zum Besser-Können « (vgl. Grawe et al., 1995, zit. n. Sickendiek et al., 1999, S. 140). Im zweiten Prinzip steht die »Klärungsarbeit « im Vordergrund, um die Motive hinter den Problemen aufzudecken. Charakteristisch dafür steht: $\gg$ Vom Nichtwissen und Nichtverstehen zum Erkennen und Sich-selbst-Verstehen « (vgl. ebd.). Im dritten Punkt, der »Problemaktualisierung «, geht es um die Erfahrbarkeit aktueller Prozesse im Hier und Jetzt, während der vierte und letzte Faktor gezielt an Potenzialen und Stärken, mit dem Schwerpunkt auf Res-

64 »Common-Factor-Forschung« bezeichnet Analysen genereller Wirkfaktoren von Beratung und Psychotherapie (vgl. Sickendiek et al., 1999, S. 137). 
sourcenaktivierung und Bewältigungspotenzialen, arbeitet. Alle vier genannten Prinzipien sind nicht ohne eine positive Wechselwirkung innerhalb der Beziehung zwischen Beratende_r und Beratene_r erfolgversprechend zu bearbeiten (vgl. Sickendiek et al., 1999, S. 140f.).

Im Wesentlichen werden die allgemeinen Interventionsstrategien von Beratung in folgenden methodischen Vorgehensweisen kategorisiert: Amplifizieren, Unterbrechen von Handlungsketten, Vereinfachen, Konfrontieren, Selbstaktivieren, Modellieren, Attribuieren, Rückmelden und Akzentuieren (vgl. ebd., S. 142). Grundsätzlich ist bei der Auswahl der Methoden darauf zu achten, dass sie sich nicht gegenseitig ausschließen, sondern ihr Potenzial durch verschiedene Kombinationen beweisen. Dafür müssen sie sorgsam aufeinander abgestimmt sein. Das bezieht sich präzise auf die Beratungskonstellationen, die Beziehungsgestaltung der Akteur_innen, das Setting und auf das konkrete Beratungsanliegen (vgl. ebd.). Auch das Verhalten von Berater_innen kann methodisch verhandelt werden. Auf vier Ebenen werden Formen des nichtverbalen (Mimik, Gestik, Körpersprache), verbalen (offene/geschlossene Fragen, Ermutigungen, Interpretationen) und verdeckten Verhaltens (unausgesprochene Strategien) diskutiert und unter spezifischen psychotherapieorientierten Beratungskonzepten zusammengefasst (vgl. ebd., S. 143). Diese Konzepte orientieren sich in ihren Grundsätzen an der sogenannten »komplexen Berater_innenreaktion «, die als Basis zur Konstituierung von Aus- und Weiterbildungen angesehen wird. Konkret beinhaltet sie die Kenntnis und Handhabung der Paraphrasetechnik ${ }^{65}$, der Wiederholung und Reflexion, sie greift nichtverbales Verhalten auf und mündet in der Zusammenfassung einer einzelnen Sitzung und/oder eines kompletten Beratungsverlaufs (vgl. ebd., S. 147).

\section{Professionalisierung}

Psychosoziale Beratung für Inters* steht vor der Herausforderung, einerseits geeignete Strukturen zu implementieren, die spezifisch auf die Bedürfnisse intergeschlechtlicher Menschen zugeschnitten sind, andererseits der Thematik insgesamt

65 Die Technik des Paraphrasierens ist die wohl bekannteste Form verbaler Beratung und hat als festes Fundament innerhalb verschiedenster Beratungskonzepte ihren Platz, beispielsweise im Ansatz klient_innenzentrierter Beratung. Die vier Formen des Paraphrasierens »signalisieren aufmerksames und einfühlsames Zuhören und leiten zur Selbstexploration an, sie ermöglichen zudem, das Beraterverständnis vom Klienten selbst korrigieren zu lassen« (vgl. Sickendiek et al., 1999, S. 148). 
gerecht zu werden und diese in einen größeren Kontext einzubetten. Beratung kann demnach nicht losgelöst von gesellschaftlichen Rahmenbedingungen betrachtet werden. Bereits der Blick auf die Einsatzfelder psychosozialer Beratung lässt eine Spannweite an unterschiedlichen Hilfe- und Unterstützungsformen erkennen, wodurch psychosozialer Beratung die Funktion einer multidimensionalen Querschnittsarbeit zukommt. Ihre Aufgabenfelder lassen sich als heterogen segmentiert charakterisieren und variieren hinsichtlich Selbstverständnis, Beratungsstrukturen und der Ausgestaltung von Beratungsprozessen (vgl. Schiersmann \& Thiel, 2004, S. 903). Generell kann zwischen personen-, gruppen- und organisationsbezogenen Einsatzfeldern unterschieden werden. Ihre sozialen Konstellationen differieren in Bezug auf plurale Beziehungsgefüge (Einzel-, Gruppen-, Paar- und Familienangebote), auf Strukturen (Beratungsformate) und formale Systeme (Organisationen, Selbsthilfe) (vgl. Sickendiek et al., 1999, S. 135). Weiterführend betrifft psychosoziale Beratung unterschiedliche soziale Versorgungsund Beratungsbereiche. Während klassische Tätigkeitsfelder der Kinder-, Jugend-, Erwachsenen- und Altenarbeit unter dem Aspekt der Alters- und Zielgruppenspezifik gefasst werden, sind darüber hinaus auch themenbezogene Schwerpunktsetzungen vielfältig miteinander verschränkt (Erwerbslosen-, Migrations- oder Suchtberatung etc.). Als zentrale Aspekte werden Tätigkeiten in den Bereichen Schule und Bildung, allgemeine Lebensführung, Sozialrecht, Pflege, Jugendhilfe, Versorgungs- und Vernetzungstätigkeiten angesehen (vgl. Nestmann, 2008, S. 74).

Zur Umsetzung geeignet scheinen vor allem Modelle, in denen der Beratungsprozess immer auch ein Beziehungsprozess ist. Das erfordert zuallerst anzuerkennen, dass es sich beim professionellen Beraten um ein kooperatives Miteinander auf Augenhöhe handelt (Teamarbeit). Dabei geht ein professionelles Handeln über das erworbene Fachwissen hinaus und bezieht persönliche und spezielle Fähigkeiten und Geschicke der beratenden Person in den Prozess ein. Erst dadurch gewinnt Beratung an Flexibilität und Individualität und verliert den Ausdruck einer schematischen Abhandlung. Der jeweils eigene Beratungsstil wirkt immer, wenn auch unbewusst oder intuitiv, auf das Gegenüber. Um dieses gefühlsmäßige (intuitive) Wissen als Teil einer Professionalisierung im Arbeitsfeld für Beratungen nutzbar zu machen, muss es geeignete Rahmenbedingungen geben, die eine Reflexion und einen gegenseitigen Austausch ermöglichen. Dies kann in Form gesundheitserhaltender und qualitätssichernder Maßnahmen erfolgen, zum Beispiel durch Supervision, kollegiale Beratung, Mediation oder Methoden und Zugänge des Psychodramas, um an dieser Stelle eine Auswahl an Möglichkeiten zu nennen. Durch die Kommunikation im Team werden die Ergebnisse für die interdisziplinäre Zusammenarbeit bewusst und nachvollziehbar. Bleibt eine Re- 
flexion dessen aus, erhält dieses individuelle und intuitive Handeln und Verhalten nicht selten den Anstrich von Zufall oder Beliebigkeit.

Um eine Professionalisierung inter*-spezifischer Beratung zu gewährleisten, sind insbesondere drei Ebenen zu berücksichtigen: informative (Sachebene), situative (Alltags- und Lebensweltorientierung) und biografieorientierte ${ }^{66}$ Beratung (vgl. Schiersmann \& Thiel, 2004, S. 899). Werden die genannten Ebenen in der Praxis miteinander verzahnt, kommt man einer ganzheitlichen Betrachtung der Beratungssituation von Inter*-Personen und des gesamten Beratungsprozesses am nächsten. Unabhängig von der äußeren Rahmung zeichnet sich ein professionalisierter Umgang innerhalb des Beratungsspektrums nicht zuletzt durch ein spezifisches Kompetenz-Design aus. Kernkompetenzen professioneller Beratung umfassen $\gg$ Hard Skills « und »Soft Skills «. Den Hard Skills sind gleichermaßen psychosoziales Wissen (Beratungsmethoden und -strategien) wie auch nichtpsychologisches Sachwissen (z. B. Informationen über Personenstandsänderung) zuzuordnen (vgl. ebd., S. 899). Grundsätzlich vereinen sie Techniken, die erlernt und angeeignet werden können und agieren daher im Außen, während als Soft Skills jene Fähigkeiten bezeichnet werden, die nicht in Form von Weiterbildung oder Studium erlern- oder messbar sind (z. B. Intuition oder Empathie). Soft Skills sind eng an eigene Persönlichkeitsanteile gekoppelt und auf dem Weg der Selbsterfahrung zu erwerben. Dementsprechend agieren sie von innen heraus.

Auch in der wissenschaftlichen Forschungsliteratur wird in Bezug auf Beratung der Erwerb dezidierter Kompetenzen und Qualifikationen dringend empfohlen. Ferner heißt es sogar, dass eine beraterische Berufsausbildung allein nicht als ausreichend empfunden wird. Als besonders wertvoll wird neben berufsbegleitenden Fortbildungen auch der Erfahrungsaustausch unter Kolleg_innen angesehen (vgl. Sanders, 2004, S. 800). Neben der grundlegenden Fähigkeit des einfühlenden Verstehens (Empathie) und dem generellen Kriterium der Verlässlichkeit (vgl. Thiersch, 2004b, S. 706) zählen kommunikative, reflexive, systemische und kooperative Kompetenzen zu den Grundlagen einer professionell handelnden Beratungsperson (vgl. Schiersmann \& Thiel, 2004, S. 900). Bestimmte Schlüsselkompetenzen (Kontextualisieren, zielbewusstes Handeln, selbstgesteuertes Lernen, soziale Kompetenzen) befruchten professionelles Beraten zusätzlich (vgl. Stark, 2004, S. 540). Neben fachspezifischem Wissen (z. B.

66 Biografieorientierte bzw. narrative Beratungsansätze sind in die inter*-spezifische Beratung einzubeziehen, da es eine heilsame Erfahrung für Inters* sein kann, ihre Geschichte zu erzählen bzw. erzählen zu können und dabei das Gefühl der Wertschätzung zu erfahren. In der Regel haben die meisten Inters* diesbezüglich in herkömmlichen Kontexten bislang wenig positive Erfahrungen gemacht. 
umfassende Kenntnisse zu Lebenslagen von Inter*-Personen und zu Inter*-Diagnoseschlüsseln) kann eine vertiefende Reflexion der eigenen Sexualität und Geschlechtsidentität der Berater_in für das professionelle Handeln hilfreich sein und zugleich die Psychodynamik zwischen den Akteur_innen begünstigen. Diese Ansicht bezieht sich im Wesentlichen auf die Anerkennung eigener identitärer Anteile, die sich unter Umständen nicht kohärent zum sonstigen Lebens- oder Liebensstil verhalten. Konkret heißt dies für die praktische Umsetzung, im Falle gleichgeschlechtlicher Lebensweisen eigene homoerotische Vorlieben oder Fantasien weder zu verleugnen noch abzulehnen.

Im Folgenden stelle ich drei exemplarische Themenfelder im Kontext inter*-spezifischer Beratung vor und untersuche diese eingehender. Mit Blick auf Kriterien der Professionalisierung stellt sich die Frage, welche Schwerpunkte speziell bei der Inter*-Beratung von besonderer Bedeutung sind. Es geht mir darum, vom Theoretischen zum Spezifischen zu gelangen. Als eine neue Sichtwiese hinsichtlich psychosozialer Beratung setze ich dem Paradigma der professionellen Distanz das Konzept der professionellen Nähe entgegen. Im Anschluss daran erfolgt eine herrschaftskritische Betrachtung der Beziehung zwischen beratender und beratener Person unter egalitären und emanzipatorischen Ansprüchen. Mit dem Wissen über diese beiden Arbeits- und Beratungsansätze wird der Fokus auf mögliche Rahmenbedingungen eines Erstgespräches mit Eltern gelegt, die ein intergeschlechtliches Kind geboren haben. Abschließend werden Anregungen für die multidimensionale Betrachtung und Entwicklung sogenannter Standards of Care generiert.

\section{Das Konzept professioneller Nähe}

Das Spannungsfeld zwischen professioneller Distanz zum eigenen Schutz und Empathie gegenüber Beratenen stellt eine schwierige Herausforderung für in der Sozialen Arbeit und der Beratung tätige Menschen dar. Sich in die (Lebens-)Situation von Inters* hineinzuversetzen kann helfen, einen adäquaten Umgang in Sprache und Verhalten zu wählen, und beugt der Gefahr einer Retraumatisierung im Beratungsprozess vor. Sich aufgrund einer möglichen emotionalen Involviertheit schützen zu wollen, um Neutralität zu bewahren und weiterhin professionell im Arbeitsfeld zu agieren, erscheint gleichermaßen nachvollziehbar und legitim. Doch geht es im Kontext von Intergeschlechtlichkeit und Beratung um einen Arbeitsansatz, der klar auf den Prinzipien von Parteilichkeit und Integrität fußt, sodass der Ansatz professioneller Distanz dementsprechend überdacht 
werden muss. Diesem Konzept wird somit der Ansatz der professionellen Nähe gegenübergestellt. Aus der kritischen Sozialen Arbeit kommend untersucht und diskutiert dieses Konzept den Aspekt des Nähe-Distanz-Verhältnisses in der Beziehungsgestaltung zwischen Berater_in und beratener Person. In der alltäglichen Beratungsroutine, insbesondere im Bereich niedrigschwelliger Sozialarbeit, wird zunehmend darauf verwiesen, professionelle Distanz zum »Klientel « zu wahren, um sich von Inhalten und Situationen innerhalb des Arbeitskontextes abzugrenzen, die im Nachgang als belastend empfunden werden können, bzw. »wenn die Problemlagen der KlientInnen den Rahmen des gängigen Repertoires sprengen « (Dörrlamm, 2006, S. 155). Eine Distanzierung soll als Burn-out-Prävention die eigene Arbeitsfähigkeit sichern und wird demzufolge als Bestandteil professionellen Handelns angeführt. Jedoch erleben Beratene dieses Paradigma nicht selten als entwertend und zur Abwehr ihrer Ansprüche herangezogen. Hingegen lässt sich, indem professionelle Nähe einbezogen wird, die Balance von Nähe und Distanz in höherem Maß verhandeln. So plädiert Dörrlamm für eine Abkehr von der Distanz als zentralem Merkmal und für Nähe als Zentrum sozialarbeiterischer und beraterischer Überlegungen:

»Aus meiner Sicht findet erfolgreiche Soziale Arbeit nur durch ein überschießendes Moment statt, das die Bedürfnisse und Notwendigkeiten der Menschen, mit denen sie arbeitet, über die bestehenden Verhältnisse stellt « (Dörrlamm, 2006, S. 156).

Als Kernaspekte professioneller Nähe dienen vorrangig sowohl ein ständiges Hinterfragen aktueller Gegebenheiten als auch eine generelle Absage an Ausschlussmechanismen jeglicher Differenzkategorien. Demzufolge kritisiert Dörrlamm die (schein-)affirmative Haltung des Konzeptes professioneller Distanz:

»Faktisch konstituiert sie eine Soziale Arbeit, die nur noch die Zurichtung der KlientInnen erreichen will. Sie sollen wieder in Strukturen passen und passend gemacht werden, aus denen sie zuvor ausgeschlossen wurden. Erst durch diese Ausgrenzung werden sie schließlich zu KlientInnen gemacht. Scheitert dieser Versuch der Zurichtung, so sind weder die bestehenden Verhältnisse noch die in der Sozialen Arbeit Tätigen, sondern ausschließlich die widerständigen KlientInnen das Problem« (Dörrlamm, 2006, S. 157).

Dörrlamms grundlegende Ansicht verweist auf eine anti-hierarchische Beziehungsgestaltung, die mit der Anerkennung bestehender Verhältnisse als Ausgangspunkt Sozialer Arbeit einhergeht und ein beratendes Vorgehen auf Au- 
genhöhe befürwortet. Die Möglichkeit, sich neuen Perspektiven zu öffnen und traditionelle Handlungsmaximen infrage zu stellen, soll nicht relativieren, dass es innerhalb der Beratung zu Grenzsituationen kommen kann. Ziel ist es, genau diese Situationen wahrzunehmen und zu reflektieren, um gemeinsam einen neuen Weg einzuschlagen - kritisch, zukunftsorientiert und nachhaltig. Nähe und Distanz bleiben somit flexibel und gewährleisten eine permanente Selbstüberprüfung der eigenen Haltung als Berater_in. Aus diesem Grund betont Dörrlamm, wie wichtig es für die Beziehungsarbeit ist, dass professionell Tätige Verantwortung übernehmen und als greifbare und authentische Personen in Erscheinung treten. Als Voraussetzung für das Konzept professioneller Nähe wird angesehen, » was gewöhnlich unter Professionalität gefasst wird. Vom umfassenden Überblick über die tatsächlichen Möglichkeiten des Hilfesystems über die Notwendigkeit, sich arbeitsfähig zu halten, bis zum Aufzeigen der aktuellen Defizite des Hilfesystems « (ebd., S. 159). Die Leitfrage, was das persönliche Handeln mit dem Ziel der eigenen Arbeit zu tun habe, erfordert die permanente Reflexion individueller, institutionell verankerter und gesellschaftlicher Bedingungen, und zwar immer im Hinblick auf den Umgang mit dem Gegenüber und der eigenen Rolle (vgl. ebd., S. 159f.).

\section{Die Beratungsbeziehung}

Das Aufeinandertreffen von beratenden und beratenen Personen geschieht im Rahmen eines institutionellen Zusammenhangs. Es ist davon auszugehen, dass ein Kennenlernen der Beteiligten im Alltag eher unwahrscheinlich ist. Die Art der Beziehung ist demnach kontextgebunden und benötigt grundlegende Überlegungen dazu, wie eine Beziehung zwischen Beratenen und Beratenden aussehen und gestaltet werden muss, um eine gemeinsame, zuverlässige und vertrauensvolle Basis zu erzielen. Der_die Vertreter_in der Kampagne Dritte Option beschreibt ihre_seine Wunschvorstellungen im Hinblick auf die Beziehung zwischen den Akteur_innen folgendermaßen:

$\gg[$ Das $]$ Eine, was wichtig wäre, ist, [...] dass es nicht so schnell darin übergeht, dass eine Person mir sagt, was ich machen soll, oder mir irgendwelche Tipps/[...] Empfehlungen oder Aufforderungen gibt, sondern tatsächlich einfach erst mal ganz viel auch zuhört, das andere [ist], [...] dass sie sich selber auch ein bisschen öffnen,[...] dass die Beratungsperson [...] mir auch einfach als [...]Mensch quasi gegenübertritt irgendwie, $[\ldots]$ was man halt einfach sehen muss, ist, $[. .$.$] wenn ich bestimmte Sachen$ 
von mir erzähle, dann[...] fühlt sich das für mich so an, als würde mich das irgendwo auch verletzlich oder angreifbar machen. Und das heißt halt quasi, dass[...] ich eine gewisse Form von [...] Vertrauen finden muss sozusagen. Und, das hat, glaube ich, dann auch ganz viel auch mit Kleinigkeiten zu tun, wie so eine Person mir gegenübertritt. Also, vielleicht auch eine gewisse Ausstrahlung, die ich halt irgendwie als akzeptierend oder als irgendwie empathisch oder so halt irgendwie wahrnehme, und das andere wäre, glaube ich schon, dass die Person einfach das ganz gut nachvollziehen kann oder nachempfinden kann, wie sich halt was für mich anfühlt. Ich glaube halt einfach, dass so ein Teil, warum dann so Ärztinnen oft irgendwie so komisch reagieren, halt einfach ist, dass die irgendwie gar nicht darüber nachdenken sozusagen, was das halt mit einem Menschen irgendwie vielleicht macht, wenn man da irgendwie so mit bestimmten Begriffen um sich schmeißt oder so, und ja, weiß nicht, wie das herzustellen ist, [...] das ist natürlich schön, wenn es da, wo es Beratung gibt, $[\ldots]$ auch so ein bisschen nett ist, $[\ldots]$ nach meiner Wunschvorstellung hat die Beratungsperson zum Beispiel auch Zeit, das haben halt Leute in Medizin in der Regel auch nicht, weil da halt noch so ein ganzes Wartezimmer sitzt so. Aber ich brauch da halt vielleicht Zeit irgendwie für mich, um dann halt bestimmte Sachen zu erklären« (Kampagne Dritte Option, 2014b, S. 13).

Es sind vier Faktoren, die zum Aufbau und zur Sicherung einer solchen Basis von Bedeutung sind: Zeit, Vertrauen, Authentizität und Empathie. Eine solche Basis kann sich im Beratungsverlauf durch das Aufeinander-Beziehen zu einer dynamischen Beziehung zwischen Beratenden und Beratenen entwickeln. Sie gehen Aushandlungsprozesse miteinander ein und stehen durch die eingeschlagene Richtung sowie die permanente Überprüfung von Beratungsanliegen und -zielen im ständigen Austausch miteinander. Nach Mahoney kennzeichnet eine optimale Beratungsbeziehung »Sicherheit, offene Entwicklungsmöglichkeiten und [...] ein grundlegendes Interesse des Beraters für die Anliegen und Probleme des Klienten « (vgl. Mahoney, 1991, zit. n. Sickendiek et al., 1999, S. 152). Die Qualität der Beziehung zwischen den Akteur_innen ist ausschlaggebend für den Beratungserfolg (vgl. Sanders, 2004, S. 806). Nach den Kriterien einer optimalen Beratung muss Beratung da ansetzen, wo der_die Ratsuchende selbst einen Schwerpunkt setzt und den dringlichsten Auftrag formuliert, und nicht dort, wo der_die Berater_in ihn vermutet. Wenn also im Interview der Faktor Zeit benannt wird, um eine vertrauensvolle Atmosphäre zu schaffen, muss dies unbedingt in der Beziehungsgestaltung Berücksichtigung finden. Berater_innen müssen sich bewusst sein, dass sie es oft mit Personengruppen zu tun haben, die unter Umständen schwer traumatisiert sind: 
$\gg$ [Meistens] haben wir es ja mit Opfergruppen zu tun. Menschen die [...] meistens medizinische Interventionen hinter sich haben, die sich [...] a) nicht selbst entschieden haben, und b) bei denen es jede Art von informierter Einwilligung nicht gegeben hat «(Veith, 2014, S. 13).

Veith erkennt eine mögliche Interdependenz zwischen Beratung und dem Hervorrufen einer (Re-)Traumatisierung:

$\gg[$ Ich] glaube, das ist [...] eine Gefahr, die auch unterschätzt wird, diese retraumatisierenden Gespräche und diese Krisen,[...] die so eine Beratung auslösen kann. Darauf muss man sehr sorgsam achten. [Ich] vereinbare zum Beispiel bei meinen Gesprächen immer ein paar Regeln vorher. [...] [Und] ich erkläre auch, was ein Trauma ist, an der Retraumatisierung «(Veith, 2014, S. 19).

Auch persönlich habe Veith bereits unsensible und grenzüberschreitende Beratungssituationen erfahren:

$\gg[$ Ich $]$ habe auch schon erlebt, dass [...] eine Beraterin [...] mich für Sachen abgefragt hat, ohne dass [...] ihr eigentlich bewusst war, was sie mich da eigentlich fragt, was sie da eigentlich in mir auslöste, welche Bilder sie auch wieder in mir auslöste, etwas, was ich sauber verpackt hatte, [...] weil ich das jetzt nachspüre, ohne das zu wollen.[...] Ja, [in einer anderen Situation habe ich] erlebt, [...] dass man Sachen angesprochen hat, die ich dann aber nicht bestätigt habe, weil ich noch gar nicht so weit war, dass ich darüber reden konnte. Und hätte man [...] mir diese Frage vier Wochen später noch mal gestellt, [...] dann hätte ich das bestätigt. [...] Da war das Vertrauen nicht ausreichend. Denn die meisten intersexuellen Menschen, die zum Opfer geworden sind, denen fehlt ja mal eine Geschichte ganz grundlegend, nämlich das [...] Vertrauen « (Veith, 2014, S. 19).

Infolgedessen muss die Beratungsperson sich die Zeit nehmen, um die Verunsicherung und den Druck abzubauen, mit dem Inters* in die Beratung kommen. In manchen Fällen stellt es für die Inter*-Person die letzte Möglichkeit dar, Zuversicht zu erlangen. So merkt Lucie Veith im Hinblick auf die hohe Verantwortung der Berater_innen an:

$\gg[$ Ich muss] wahrnehmen, [...] dass ich einen Menschen in einer Krise da habe. [...] Es ist meistens auch eine schwere Krise. [...] [Wenn] dieser Mensch nie über sich und über diesen Zustand geredet hat, dann muss ich [in] jedem Fall auch den Suizid 
immer im Hinterkopf haben. Dass das nämlich dann vielleicht auch noch mal [...] der letzte Versuch ist, noch mal etwas zu klären, und wenn das nicht klappt, eigentlich schon die Gewissheit zu haben, diese letzte Option zu wählen. Denn meistens ist es ja so, dass wir nur zwei Optionen sehen, wenn wir so dual denken. Und dann ist es tatsächlich so, entweder die helfen mir jetzt oder es hat sowieso keinen Zweck. Das haben wir ganz oft « (Veith, 2014, S. 6f.).

Ähnliche Erfahrungen werden auch aus dem Bereich der Homosexuellen-Beratung beschrieben:

»Suizidalität ist in diesem Zusammenhang ein wichtiges Thema, etwa wenn die Klientin oder der Klient ihre bzw. seine unerwünschte, vielleicht doch noch ganz uneingestandene Homosexualität als dermaßen identitätsbedrohend erlebt, dass er keinen anderen Ausweg [sieht], als sich das Leben zu nehmen « (Wiesendanger, 2004, S. 252).

Das Schaffen einer einladenden, angenehmen und ruhigen Atmosphäre bildet gerade zu Beginn einer Beratungssequenz eine sichere Grundlage. Die Dimensionen von Beratung müssen dem_der Beratenen transparent sein (Beratungsbeziehung, Beratungsgrundlagen, darunter u.a. methodisches Vorgehen). Beratungsarbeit muss personenzentriert sein, sich an Heterogenität orientieren und sensibel in Bezug auf intersektionale Verschränkungen (Klassen-, Geschlechter- und rassistische Verhältnisse) reagieren. Teilaspekte der Identität (z. B. sexuelle Orientierung, Armut, Religion) dürfen dabei nicht per se als zentraler Beratungsinhalt gesetzt werden. Vielmehr sind sie als Zuschreibungen zu verstehen, die von außen getätigt werden. Ziel und Inhalt von Beratung ist es, sich selbst in den eigenen Urteilen zurückzunehmen und empfänglich für das zu sein, was seitens der Ratsuchenden tatsächlich als problematisch benannt wird. Homosexualität ist nicht zwangsläufig Beratungsinhalt, nur weil bekannt ist, dass die betreffende Person homosexuell lebt - insbesondere wenn das Anliegen eigentlich ein ganz anderes ist. Da Beratung sehr komplex strukturiert ist und sich durch diverse Beratungsfelder, -ebenen und Zielgruppen auszeichnet, wird eine konkrete Klärung des Auftrags umso wichtiger:

$\gg$ Also ich denke, [...] ein weiterer ganz wichtiger Punkt ist eben tatsächlich, dass man $[\ldots]$ Inter*-Beratung immer $[\ldots]$ multidimensional denken muss und auch immer klären muss, auf welcher Basis unterhalten wir uns jetzt eigentlich. Worum geht es eigentlich? Geht es da um Personenstand? Und geht es darum, wie Leute 
dich wahrnehmen und wie du dich wahrnimmst, oder meinst du das eher in Bezug auf deine sexuelle Orientierung, also auch auf deine sexuelle Identität, wie siehst du dich, in [...] welcher Rolle siehst du dich, und in welcher Rolle wärst du gerne, und kann ich das erreichen, und wie [...] und da aber immer das Defizit rausnehmen, das ist die Kunst dabei, [...] auch den Ist-Zustand nie als Defizit wahrzunehmen « (Veith, 2014, S. 13).

Im Kontext von Intergeschlechtlichkeit ist oftmals nicht die Tatsache der eigenen Geschlechtlichkeit Grund für das Einholen von beraterischer Unterstützung, sondern die äußeren strukturellen Begebenheiten, die ein Leben als Inter* erschweren. Das zu berücksichtigen dient der Festigung einer sich entwickelnden, vertrauensvollen und sicheren Beziehungsgestaltung, deren Grundannahmen durch Akzeptanz und Offenheit charakterisiert sind. So ist es wichtig, dass sich der_die Beratende an der individuellen und persönlichen Lebens- und Deutungswelt der Beratenen orientiert und versucht, diese zu verstehen und zu respektieren (vgl. Mahoney, 1991, zit. n. Sickendiek et al., 1999, S. 152). Insbesondere bei der spezifischen Inter*-Beratung kommen die zuvor genannten Beratungs-Skills zum Tragen:

$\gg\left[\right.$ Inters $\left.^{*}\right][\ldots]$ fühlen sich [...] betrogen um ihr Leben zum Teil. Und das wieder annehmen zu können, dieses betrogene Leben, da muss man einen ganz schönen Perspektivwechsel (lacht) vollziehen, um das in einen positiven Blick wieder zu bekommen. Und [...] diese Traumata tatsächlich auch zu, [...] wie kann ich da eigentlich mit umgehen? Auch eine Technik anbieten. Das [...] muss ich draufhaben. Und das muss ich vielleicht auch mal, [...] am ersten oder zweiten Beratungstag, [...] und wahrscheinlich schon am ersten wäre es gut, wenn das ein großer Termin ist, dass man dann sagt: Und eine Sache möchte ich gerne nochmal üben, bevor wir hier ins Ganze gehen. Es kann sein, dass du dich mal schlecht fühlst. Und dann mach dir keine [...] Sorgen, dann ist das kein Angriff gewesen, sondern da wird was ausgelöst. Und das kannst du wieder einholen, wenn es dir ganz schlecht geht, dann setz dich hin, und das üben wir jetzt mal. Mal eine kleine Meditation « (Veith, 2014, S. 19f.).

Was Lucie Veith hier als »kleine Meditation « benennt, wird den Beratenen in der traumapädagogischen Arbeit unter der Bezeichnung »Reise an einen sicheren Ort « als Angebot für den Umgang mit Trigger-auslösenden Situationen vorgeschlagen. Dies dient als eine Art unsichtbares Handwerkszeug und wird nicht selten als Coping-Strategie genutzt, um einen Umgang mit retraumatisie- 
renden Situationen zu finden. Lucie Veith sieht es als absolut notwendig an, dass eine spezifische Inter*-Beratung über traumapädagogische Grundkenntnisse verfügt:

»Ich muss sie im Auge haben, wenn ich die nicht im Auge habe, geht die Beratung in die Hose. Ich muss Grundkenntnisse haben, unbedingt. Wenn ich die nicht habe, brauche ich das nicht machen. Dann [...] darf ich niemanden beraten, bei dem ich nicht hundertprozentig sicher bin, [...] dass es keine Übergriffe gegeben hat. Wenn das nicht geklärt ist, laufe ich ja immer Gefahr, dass ich irgendwas auslöse, was dann nicht kontrollierbar ist « (Veith, 2014, S. 20).

Beratung stellt im Kontext von Intergeschlechtlichkeit und vor allem im Kontext von Zielgruppen, die Gewalt erlebt haben, eine besondere Herausforderung dar. Hier geht es vor allem um Krisenintervention. Dadurch wird die Reichweite des gesamten Beratungsspektrums deutlich. Die grundsätzliche Frage nach generellen Grenzen von Beratung wirft die Überlegung auf, ob die Bearbeitung von Traumata im Bereich der Beratung angemessen umgesetzt werden kann. Lucie Veith siedelt Traumaarbeit einerseits an der Schnittstelle zwischen professioneller Beratung und Psychotherapie an, gegebenenfalls als gemeinsame Kooperationsaufgabe. Andererseits könne diese spezielle Krisenbewältigung auch in Form spezifischer traumapädagogischer Beratung getrennt von sowohl klassischer Beratung als auch von Psychotherapie als eigenständige Umgangs- und Beratungsform angeboten werden (vgl. ebd.). Wichtig ist hierbei, dass eine Bearbeitung von Traumata im Beratungsprozess spezifische und professionalisierte Kenntnisse benötigt.

Nicht alles, was im Beratungsprozess erzählt und besprochen wird, können Berater_innen leicht verarbeiten, finden sie gut oder schlecht, richtig oder falsch, nachvollziehbar oder auch nicht. Dennoch obliegt es Beratungs- und Fachpersonal nicht, Bewertungen vorzunehmen, wenn nicht explizit danach gefragt wurde (vgl. Sickendiek 2004, zit. n. Nestmann et al., 2004b, S. 772). Zu lernen, Dinge auszuhalten, ist eine wesentliche Berater_innenkompetenz. Nicht selten wird Beratenden eine Vorbildfunktion für einen vermeintlich intakten Lebensentwurf zugeschrieben. Dies kann sich zwar positiv auf das Wohlbefinden und die Widerstandsfähigkeit des Gegenübers auswirken, allerdings ist es empfehlenswert, ein realistisches und authentisches Bild von sich selbst in der Rolle der_des Ratgeber_in zu transportieren. Lernen, Grenzen und Schwächen zu akzeptieren, kann einen wichtigen Entwicklungsschritt und -erfolg im Beratungsverlauf darstellen und spiegelt darüber hinaus gesellschaftliche Realität 
wider. Selbst(für)sorge ist für alle involvierten Akteur_innen von Beratungsbeziehungen eine verantwortungsvolle Angelegenheit und eng gekoppelt an den persönlichen Selbstwert sowie an individuelle Grenzen. So stellt sich die Frage, ob die Selbstexploration im Sinne einer sprachlich-dialogischen Reflexion über persönliche innere Erfahrungen im Beratungsprozess nicht auch heißt, sozial verantwortlich zu handeln bzw. handeln zu müssen. Das gilt ebenso für die Verantwortlichkeit jedes_r Einzelnen, eigene Entscheidungen zu treffen. Mahoney benennt an dieser Stelle die Wichtigkeit dessen, sich bewusst zu sein, dass innerhalb von Beratung » mit den Widerständen der Klienten, nicht gegen sie « gearbeitet werden muss (vgl. Mahoney, 1991, zit. n. Sickendiek et al., 1999, S. 153). Offenheit und Ehrlichkeit können dabei helfen, die Schwere bestimmter Thematiken zu verringern und ihnen realistisch zu begegnen. Diese Aussage wird auch von Lucie Veith durch einen Appell an die Selbstverantwortung der_des Ratsuchenden gestützt:

»Jetzt kann ich natürlich sagen, das ist ganz traurig, was du da erlebt hast, und das teilst du mit ganz vielen Menschen, die kannst du [zum Beispiel in der Selbsthilfe] auch kennenlernen, auf der anderen Seite, egal, was man mit uns macht, wir bleiben immer wir selbst. [...] Du hast das Recht darüber zu weinen und auch traurig zu sein, aber auf der anderen Seite hast du auch das Recht, dein Leben in die Hand zu nehmen, [...] jetzt darfst du beginnen, deinen Lebensentwurf zu bauen. Leider ist nicht mehr alles möglich, ein bisschen was hat man dir genommen, aber wir können ja mal [...] gucken, inwieweit deine Wünsche und deine Vorstellungen da noch erfüllt werden können. Das könnte man mal erarbeiten, so würde ich da herangehen, zum Beispiel « (Veith, 2014, S. 13).

Die Förderung von Empowerment und Selbstbestimmung ist wesentliches Grundprinzip emanzipatorischer Beratungskonzepte, das vor allem auch im Hinblick auf die Zielgruppe der Inters* Berücksichtigung finden muss. EmpowermentProzesse operieren auf individueller, struktureller und auf Gruppen- und Organisationsebene (vgl. Stark, 2004, S. 537). Zielsetzung ist die Integration intergeschlechtlicher Menschen als aktive Subjekte in den Gesamtprozess mit Blick auf deren selbst-aktivierende Potenziale (vgl. Sickendiek et al., 1999, S. 71). Der Begriff des Empowerments intendiert »eine Form der weitestgehend selbstbestimmten Partizipation, die nicht expertendefiniert von außen gesetzt wird, sondern sich aus der Perspektive der direkt Betroffenen konstituiert « (vgl. Sickendiek et al., 1999, S. 72). Empowerment ist integraler Bestandteil von Beratung (vgl. Stark, 2004, S. 535), geht aber über die Grenzen persönlicher Problembe- 
wältigung hinaus und ist dadurch auch auf politischer Ebene wirksam (vgl. Vogt, 2004, S. 216).

\section{Das Eltern-Erstgespräch}

»Die Medizin ist seit meiner frühesten Kindheit in mein Leben involviert, genau genommen seit dem Augenblick meiner Geburt. Sie war zentral für einige meiner Probleme, sowie für einige Erkenntnisse. Ich denke, dass mein Körper durch die medizinischen Eingriffe, denen ich von Anfang an unterworfen war, sehr verletzlich wurde und die erlittenen Traumata bis heute nicht vergessen hat « (Sebastian, 2013, S. 11f.).

Während zuvor das Hauptaugenmerk auf dem Personenkreis lag, der aus eigener Initiative heraus Beratungsangebote aufsucht, wird nun die Zielgruppe sehr junger Inters* und ihrer Eltern in den Blick genommen. Ausgehend von der Tatsache, dass Inter*-Kinder bereits im frühen Alter medizinische Gewalt erleben, besteht diesbezüglich erheblicher Beratungsbedarf - zum einen gegenüber den Eltern, zum anderen, kindgerecht, gegenüber den Inters*. So konstatiert Kate Bornstein:

$\gg$ The first question we usually ask new parents is: >Is it a boy or a girl? <. There is a great answer to that one going around: >We don't know; it hasn't told us yet.< Personally, I think no question containing > either/or < deserves a serious answer, and that includes the question of gender $\ll$ (Bornstein, 2014).

( $\gg$ Als erstes fragen wir neue Eltern normalerweise: $>$ Ist es ein Junge oder ein Mädchen?<. Auf diese Frage ist eine großartige Antwort im Umlauf: >Wir wissen es nicht, es hat uns das noch nicht verraten. $<$ Ich persönlich finde, dass keine EntwederOder-Frage eine ernsthafte Antwort verdient, und die Frage nach dem Geschlecht ist eine davon «) (eigene Übersetzung).

In der Regel erfahren Eltern frühestens zum Zeitpunkt der Geburt, dass ihr Kind intergeschlechtlich ist bzw. keinem Geschlecht eindeutig zugewiesen werden kann. Umso größer ist anschließend die Verunsicherung und Überforderung, was Intergeschlechtlichkeit für sie als Eltern, für ihr Kind und das gemeinsame Familienleben bedeutet und mit welchen konkreten Auswirkungen sie sich konfrontiert sehen (vgl. Sabisch, 2014, S. 57; IMeV, 2009, S. 3). Zunächst sorgen sich Eltern oft um ihr Kind und seine Gesundheit. In einem nächsten Schritt entstehen 
Gefühle von Angst und Unsicherheit vor den Reaktionen des sozialen Umfeldes und der Gesellschaft. Sowohl die anfängliche Unsicherheit der Eltern als auch die des erweiterten Nahraums ist vorwiegend auf ein Unwissen über Intergeschlechtlichkeit zurückzuführen. Somit scheint es Aufgabe von Beratungspersonen zu sein, sich dieser Situation anzunehmen und »Brücken zu bauen «, um Eltern zu einem offenen Umgang mit der Intergeschlechtlichkeit ihres Kindes zu befähigen. Der Zugang zu Fachinformationen stärkt eine akzeptierende Grundhaltung und unterbindet Ablehnung und Irritation. Diese Erkenntnis und auch der unbefangene Umgang von Kindern mit ihrer eigenen Geschlechtlichkeit befördern eine generelle Veralltäglichung von Intergeschlechtlichkeit (vgl. Sabisch, 2014, S. 58f.). Hier gilt es, die Eltern zu ermutigen und ihre Ressourcen dahingehend zu stärken. Intergeschlechtlichkeit muss im Alltag normalisiert behandelt werden, was ein regelmäßiges »Darüber-Sprechen « zwischen Eltern und Kind erfordert.

Nicht selten fühlen sich Eltern unter einem Rechtfertigungsdruck, sowohl gegenüber dem engeren Bekanntenkreis als auch gegenüber medizinischen Instanzen, hinsichtlich der Entscheidung für oder gegen eine geschlechtszuweisende und geschlechtsvereindeutigende Operation. Die Studie zum erinnerten Erziehungs- und Bindungsverhalten der Eltern, die durch die Hamburger Forscher_innengruppe 2006 erhoben wurde, konstatiert, dass viele Inters* ihre Eltern im Rückblick als wenig fürsorglich erlebt haben (vgl. Schweizer \& Richter-Appelt, 2012b, S. 198). Als Begründung geben die Autor_innen an, »dass auch die Eltern durch die Intersexualität ihres Kindes und den lange üblichen Umgang der Geheimhaltung und schnellstmöglichen Geschlechtsanpassung verunsichert wurden und diese Verunsicherung weitergegeben haben « (ebd.). Dieses Resultat bestätigt die Annahme, dass auch der Bereich der Elternberatung einen nicht zu vernachlässigenden Faktor darstellt. Ferner stellt J. M. Pulvermüller, Mutter eines intergeschlechtlichen Kindes, die These auf,

»dass Eltern, die selber die Erfahrung einer fürsorglichen, akzeptierenden und ermutigenden Begleitung, insbesondere bei der Geburt ihres intersexuellen Kindes, erlebt haben, eine solche Haltung auch eher ihrem Kind entgegenbringen konnten als solche, denen mit Unsicherheit, Angst und Verleugnung der intersexuellen Wirklichkeit des Kindes begegnet wurde (Pulvermüller, 2012, S. 198).

Anknüpfend an diese Aussage liefert der folgende Abschnitt zwar keine allumfassende Beratungshilfe für die »Notsituation «, wenn Eltern vor der Frage eines medizinischen Eingriffs stehen, und auch keinen vollständig ausgereiften Leitfaden zur kindgerechten Aufklärung von Inters*. Dennoch wird das zielgrup- 
penspezifische Beratungsfeld der Eltern aufgegriffen und hinsichtlich zentraler theoretischer Kriterien und Rahmenbedingungen für ein mögliches Erstgespräch nach der Geburt bzw. begleitend zur Diagnosemitteilung exemplarisch durchdacht. Professionelle Handlungsansätze für die Zukunft lassen sich sowohl daraus ableiten als auch diesbezüglich ausbauen.

Der Beginn einer Beratungssequenz unterliegt keinem routiniert-festgelegten Schema. Das Beratungsgespräch selbst konstituiert sich aus dem Aufeinandertreffen der Akteur_innen und ihrem interaktionellen Handeln. Ausgehend davon stellt sich generell die Frage nach der Sinnhaftigkeit von Beratungsmanualen und Leitfäden (vgl. Nestmann et al., 2004b, S. 744). Dennoch gibt es hinsichtlich potenzieller Erstgespräche bereits Ansätze, die durchaus hilfreich für den Erstkontakt sein können. In Anlehnung an den Hebammen-Leitfaden des Vereins Intersexuelle Menschen zur Unterstützung und Entwicklung von Verhaltensweisen im Umgang mit intergeschlechtlichen Neugeborenen und ihren Eltern benennen diese Handlungsempfehlungen wichtige Aspekte, die es aufzugreifen und ebenfalls im psychosozialen Beratungsverlauf mit Eltern bzw. Familiensystemen zu berücksichtigen gilt. Übergeordnete Ziele sind einerseits, Eltern hilfreich zur Seite zu stehen, und andererseits, dem Kind ein »Leben in Würde, körperlicher Unversehrtheit und Selbstbestimmung « zu gewährleisten (IMeV, 2009, Danksagung).

Ein Erstkontakt, der innerhalb der entscheidenden Phase nach der Entbindung, meist noch im Krankhaus, stattfindet, ist immer situationsabhängig und durch viele Faktoren bestimmt. Der Geburtsverlauf, die physische und psychische Kondition der Eltern bzw. der Mutter sind Beispiele möglicher Einflussfaktoren. Darüber hinaus ist es ebenso wissenswert, ob bereits ein ärztliches Gespräch stattgefunden hat, ob und in welcher Form die Diagnose übermittelt wurde und welche fachlichen Hilfen darüber hinaus installiert sind (zum Beispiel Hebammen, Psycholog_innen, etc.). Es ist daher sinnvoll, sich mit allen beteiligten Fachdisziplinen vorab zu besprechen, um den Eltern überfordernde oder sich mehrfach wiederholende Situationen zu ersparen - eine Anregung, die für eine flächendeckende Implementierung transdisziplinärer Beratungsteams spricht. Eine goldene Regel gilt es generell bei diesem Erstkontakt zu berücksichtigen: die Schaffung einer angemessenen Atmosphäre. Die erste Beratungssituation sollte so viel Ruhe und Geborgenheit wie möglich vermitteln, Vertrauen ausstrahlen, um ohne Zeitdruck transparent und offen über die Intergeschlechtlichkeit des Kindes und die damit einhergehenden Befindlichkeiten der Eltern zu sprechen (vgl. IMeV, 2009, S. 2). Ebenso müssen Eltern über den anschließenden Prozess ausreichend in Kenntnis gesetzt werden. Denn in der Regel wissen sie nicht, 
dass ein umfassender diagnostischer Vorgang mit langen Wartezeiten verbunden sein kann. Wichtig für die Beratungsperson ist dabei, nicht voreilig aus eigenem Handlungsdruck heraus zu reagieren (vgl. Reutlinger, 2004, S. 1196). Oft braucht es Zeit, die Tragweite der neuen Situation zu überblicken. Es ist diesbezüglich sinnvoll, eine längerfristige psychosoziale Prozessbegleitung anzubieten. Auch das Einsetzen sogenannter $\gg$ Sofortpaten $\ll{ }^{67}$ für Familien mit intergeschlechtlichem Kind ist als Option durchaus denkbar und sichert, dass eine Vertrauensperson vorhanden ist. Empfehlenswert ist die parallel laufende Vermittlung sowohl zu verfügbaren Selbsthilfe- und Versorgungsstrukturen, darunter zum Beispiel Elterngruppen und/oder spezialisiertes (medizinisches) Fachpersonal, als auch zu inter*-spezifischen Kompetenzzentren (vgl. IMeV, 2009, S. 3).

Jeder $\gg$ Fall « von Intergeschlechtlichkeit ist individuell unterschiedlich. Eltern hoffen oft auf allgemeingültige, entlastende Aussagen, die ihnen in diesem Zusammenhang nicht geboten werden können bzw. sollten (vgl. ebd., S. 1). Dennoch ist es möglich, der Angst der Eltern zu begegnen, indem man sie darüber aufklärt, dass die meisten Formen von Intergeschlechtlichkeit grundsätzlich weder die Gesundheit noch die frühkindliche Entwicklung des Kindes beeinträchtigen (vgl. ebd., S. 2). In Bezug auf die Rolle der Berater_in bzw. des Beratungsteams wird angeraten, Sachlichkeit zu wahren und persönliche Bewertungen bzw. Meinungen zurückzuhalten. Sexualität und Geschlecht stellen für viele Menschen Themen persönlicher Intimität dar. Nicht jede Person ist gewohnt, offen darüber zu sprechen (vgl. ebd., S. 3). Auch in Bezug auf die professionelle Rolle des_r Berater_in müssen eigene Gefühle von Scham und Verunsicherung aufgearbeitet werden (vgl. Arbeitsgruppe Ethik, 2008, S. 244). Des Weiteren muss die Reflexion eigener verinnerlichter dichotomer Denkkategorien stattgefunden haben. In Anbetracht der genannten Gründe ist eine Sensibilisierung hinsichtlich möglicher Grenzüberschreitungen dringend geboten. Es ist ratsam, dass diese Hinweise auch an Ärzt_innen und Pflegefachkräfte vermittelt werden.

Da Erstdiagnosen häufig zu Verunsicherung und Überforderungsgefühlen bei den Eltern führen, ist eine Rückzugsmöglichkeit, beispielsweise durch das Angebot eines Einzelzimmers, zu gewähren. Somit können die Eltern ihre neue Situation in Ruhe reflektieren und wirken lassen (vgl. IMeV, 2009, S. 3). Im güns-

67 Sofortpaten entstammen meistens dem Umfeld der Selbsthilfe. Ihre Kontaktdaten liegen in den Kliniken vor, sodass sie bei Geburt eines intergeschlechtlichen Kindes eine Begleitung der Eltern anbieten können, solange diese noch mit ihrem Kind in der Klinik sind. Weitere Informationen sind beim Verein Intersexuelle Menschen e.V. zu erfragen (vgl. IMeV, 2009, S. 4). 
tigsten Fall reduziert dies sogar die empfundene Belastung der »Betroffenen « und ihrer Eltern. Von zentraler Bedeutung ist es, den Unterstützungs- und Hilfesuchenden keine vorgefertigten Kategorien überzustreifen und zu bedenken, dass gängige Muster im Zweifelsfall nicht anwendbar sind (vgl. Zehnder, 2008, S. 29). Somit ist Inhalt und Aufgabe einer Beratung, die im Sinne von Inters* agiert, von jeglichen chirurgischen Maßnahmen abzuraten, solange keine medizinische Indikation vorliegt. In Anlehnung an die Änderungen des Personenstandsgesetzes von 2013 ist zudem die Geschlechtskategorie (zunächst) offen zu lassen:

»Kann das Kind weder dem weiblichen noch dem männlichen Geschlecht zugeordnet werden, so ist der Personenstandsfall ohne eine solche Angabe in das Geburtenregister einzutragen«(Tolmein, 2014, S. 12).

Trotz der Kritik, dass diese Gesetzesänderung als Vorschrift anstelle einer Wahlmöglichkeit fungiert und keinen progressiven Paradigmenwechsel hinsichtlich der Akzeptanz geschlechtlicher Vielfalt initiiert und rechtlich stützt, ist sie zumindest hinsichtlich eines vermeintlichen Handlungsdrucks als entgegenkommende Entschleunigung ${ }^{68} \mathrm{zu}$ sehen. Denn medizinische Entscheidungen werden hier häufig überstürzt getätigt und sind in den meisten Fällen irreversibel. Aus- und Nebenwirkungen sowie Nachbehandlungen und generelle Risiken einer solchen Maßnahme sind zu diesem Zeitpunkt in der Regel nicht in vollem Ausmaß einund abschätzbar. Oft wird dabei nicht bedacht, dass geschlechtsvereindeutigende und geschlechtszuweisende Genitaloperationen wesentliche Körperfunktionen beeinträchtigen können, beispielsweise die sexuelle Empfindsamkeit (vgl. IMeV, 2009, S. 3). Deshalb formulieren Empfehlungen unter ethischen Gesichtspunkten eine abwartende Haltung und stützten die Forderungen $»$ Betroffener « nach einem Moratorium. Die Arbeitsgruppe Ethik im Netzwerk Intersexualität vertritt eine ähnliche Haltung. Sie bestätigt, dass » geschlechtliche Uneindeutigkeit per se keine behandlungsbedürftige Kondition [ist] « (vgl. Arbeitsgruppe Ethik im Netzwerk Intersexualität, 2008, S. 242).

Die Medizinier_innen, die Eltern zu einer umgehenden geschlechtszuweisenden Operation raten, stützen sich in der Regel auf das Argument der vermuteten

68 Der Begriff der Entschleunigung ist in diesem Zusammenhang durchaus kontrovers zu betrachten. Seitens der Wissenschaft und Medizin ist die Bezeichnung zutreffend, da im medizinischen Diskurs momentan der Fokus auf ausführlicherer Beratung im Sinne von Qualitätsstandards gelegt wird. Andererseits kann der Druck auf die Eltern durch diese Änderung sogar steigen, weil sie folglich nur durch medizinische Eingriffe einen eindeutigen Geschlechtseintrag erwirken können. 
Alltagsdiskriminierung der Kinder (vgl. Sabisch, 2014, S. 59). Eine wissenschaftliche Untersuchung ${ }^{69}$ zu Lebenslagen von Familien mit Inter*-Kindern zeigt aber, »dass eine offen gelebte Zwischengeschlechtlichkeit im Kindesalter keine Diskriminierungen mit sich bringen muss « (ebd.). Ferner muss sich eine psychosoziale Beratungspraxis diesbezüglich an Partizipationsrechten von Inter*-Kindern orientieren, dem Auftrag zur Sicherung ihres Kindeswohls nachkommen und den kindlichen Anspruch auf eine liebevolle und fürsorgliche Eltern-Kind-Beziehung als Handlungsauftrag unterstützen und gegebenenfalls begleiten. Demzufolge ist es hinreichend zu erkennen, dass »Eltern nicht nur als Stellvertreter ihrer Kinder in allen Behandlungsentscheidungen, sondern auch als Betroffene, die selbst Hilfe und Unterstützung benötigen « (Arbeitsgruppe Ethik im Netzwerk Intersexualität, 2008, S. 244), wahrgenommen werden müssen. Entscheidungen hinsichtlich des Kindes sind immer so zu treffen, dass das Kind auch als später erwachsene Person mitgedacht wird. Demzufolge zielen ethische Prinzipien über die Entscheidung möglicher Eingriffe vor allem auch auf körperliche Integrität, allgemeine Lebensqualität, Reproduktionsfähigkeit, sexuelles Erleben und die freie Persönlichkeitsentfaltung ab (vgl. ebd.). So heißt es in den Empfehlungen der Arbeitsgruppe Ethik:

»Bei der Beurteilung des Kindeswohls sind eine mögliche Verunsicherung und Traumatisierung durch operative Maßnahmen, Korrektureingriffe und wiederholte Untersuchungen im Intimbereich ebenso zu berücksichtigen wie eine mögliche Einschränkung der sexuellen Erlebnisfähigkeit und der Fortpflanzungsfähigkeit des zukünftigen Erwachsenen. Sie müssen gegen die Vorteile oder ggf. biologisch eindeutige Geschlechtsidentität für das Kind abgewogen werden « (Arbeitsgruppe Ethik im Netzwerk Intersexualität, 2008, S. 245).

Aus den angestrebten Beratungszielen wird ersichtlich, dass die genannten Erwartungen nicht nur in Form eines Erstgespräches abzuhandeln sind. Ein gut strukturierter und die grundsätzlichen Kriterien beachtender Erstkontakt ist jedoch nicht zu unterschätzen. Auch in nur einem Gespräch können Grundsteine gesetzt und Anstöße gegeben werden. Abhängig von individuellen Ausgangssituationen geht die allgemeine Tendenz psychosozialer Beratungsverläufe hin zu

69 Die genannte Untersuchung basiert auf drei narrativ geführten Interviews mit Eltern, die ihre Kinder in einer offenen Geschlechtskategorie aufwachsen lassen. Die analysierten Interviews wurden Anfang 2013 von Anike Krämer durchgeführt und im Rahmen ihrer Masterarbeit mit dem Titel »Inter* im Alltag. Eine empirische Forschungsarbeit zu Lebenslagen intersexueller Kinder und ihrer Eltern« an der Ruhr-Universität Bochum verfasst. 
einem Angebot längerfristiger prozesshafter Begleitung. Durch Akzeptanz und Offenheit gegenüber dem Kind kann die Entwicklung eines Selbstwertes im Hinblick auf persönliche und geschlechtliche Identität durch die Prozessberatung professioneller gewährleistet werden (vgl. ebd.). Diese Form der Unterstützung ist in übergeordnetem Kontext als Ausdruck einer Flexibilisierung der Geschlechterordnung gekennzeichnet (vgl. Sabisch, 2014, S. 59). Als elementarer Anspruch an eine gesellschaftlich reflektierte Herangehensweise versteht sich folgendes $\mathrm{Zi}$ tat der Arbeitsgruppe Ethik:

»Im Zweifel hat die psychische und soziale Unterstützung des Kindes und seiner Eltern einen höheren Wert als die Herstellung einer biologischen Normalität « (Arbeitsgruppe Ethik im Netzwerk Intersexualität, 2008, S. 245).

Weiterhin wird dem Kind bei einer Entscheidung gegen eine operative Maßnahme die Option zur Ausprägung einer eigenen selbstgewählten Geschlechtsidentität bzw. ein Wechsel der Geschlechtsidentität ermöglicht. Den Eltern sollte daher auch bereits im Erstgespräch angeboten werden, bei »prognostisch unsicheren Maßnahmen bis zur Entscheidungsreife des Kindes « (ebd.) abzuwarten. Das gilt ebenso für die Entnahme der Keimdrüsen. Besteht auch in diesem Kontext keine medizinische Indikation, so muss die Verfügung darüber den Inters* selbst überlassen werden. Können sie hierüber aufgrund ihres Alters noch nicht entscheiden, muss auch in diesem Fall bis zur Entscheidungsreife gewartet werden. Aktuell haben Inters* die Möglichkeit einer selbstbestimmten Entscheidung bereits beim Eintritt in das 14. Lebensjahr (vgl. ebd.). Vorher gilt es - und ist es somit auch grundlegender Handlungsauftrag von Beratung -, die Teilhaberechte des Kindes in vollem Maße zu berücksichtigen und diese kindgerecht umzusetzen. Dazu gehört das Anrecht auf vollständige Aufklärung in kindgemäßer Sprache und Form. Ebenso zählt dazu, bezüglich medizinischer Maßnahmen bereits am Willen des Kindes erkennbare Entscheidungen zu respektieren. Voraussetzung dafür ist, von Beginn an und konstant die Meinung des Kindes zu erfragen und es in Untersuchungs- und Behandlungsschritte einzubeziehen (vgl. ebd.).

Weniger existenziell für das Erstgespräch, aber dennoch elementar wichtig für die grundlegende Lebensqualität ist eine lückenlose Dokumentation des medizinischen Verlaufs, die im Erwachsenenalter von den betreffenden Inters* selbst jederzeit eingesehen werden kann - selbstverständlich unter Gewährleistung der Datenschutzbestimmungen und einhergehend mit einer ethischen Begleitforschung, die die Zufriedenheit der Inters* hinsichtlich der Begleitung und Beratung im transdisziplinären Team evaluiert (vgl. ebd.). 


\section{Anregungen zur Entwicklung von »Standards of Care»}

Tabuisierung, Verunsicherung und Geheimhaltung sind Erfahrungen von Inters*, die für den Beratungsprozess von großer Bedeutung sind. Grundsätzlich ist von der Annahme auszugehen, dass Inters* die schwerste Hürde bereits überwunden haben, wenn sie sich auf psychosoziale Beratungsinstanzen einlassen (vgl. Reutlinger, 2004, S. 1194). Durch jahrelange offene und strukturelle Gewalt- und Diskriminierungserfahrungen haben sie Coping-Strategien entwickelt, die ihnen in gewisser Weise Schutz geboten haben. Eine Möglichkeit des Umgangs schildert Ins A Kromminga, wenn er_sie aus eigener Erfahrung und gestützt auf den Austausch mit anderen Inters* berichtet:

»Der Humor, wenn auch häufig finster und zynisch, erleichtert den Zugang zu Themen, die in all ihrer Heftigkeit schwer aushaltbar oder sehr komplex sein können $\ll$ (Kromminga, 2013, S. 102).

Oft sind Inters* noch nicht einmal Formen informeller Beratung, zum Beispiel im familiären Rahmen, zuteil geworden. Deshalb erfordert bereits der Erstkontakt ein hohes Maß an Sensibilität. Nicht immer ist es dabei ratsam, direkt an spezialisiertere Beratungsinstanzen weiterzuvermitteln, da dies von Ratsuchenden oft als »Abschiebung « und als Verlust einer Vertrauensperson wahrgenommen wird:

»[Oftmals] erfahren KlientInnen zwar ein Ernstgenommenwerden, aber dann gleichzeitig ein Abschiebenwollen zu professionelleren BeraterInnen « (Reutlinger, 2004, S. 1195).

Daher ist zu prüfen, »ob die Beratung nicht doch mithilfe von oder in Begleitung durch FachberaterInnen auch von der Vertrauensperson selbst gewährleistet werden kann « (ebd.). Dies erfordert eine unterstützende Vernetzung sozialer und psychologischer Hilfen, deren Konsolidierung als grundlegende Handlungsmaxime anzustreben ist, und den Aufbau solidarischer Kooperationen und Arbeitsbündnisse. Beratung und Begleitung mit von Gewalt Betroffenen bilden wichtige Arbeitsschwerpunkte, deren Grundelemente Offenheit und Transparenz sind. Neben dem Grundsatz der Freiwilligkeit zum Aufbau vertrauensvoller Beziehungen ist auch der Bereich der individuellen Grenzsetzung zum Herausfinden des geeigneten Nähe-Distanz-Verhältnisses sensibel zu erarbeiten. Der ehrliche Umgang mit den eigenen Grenzen kann auch eine positive Vorbildfunktion haben. 
Er ermöglicht Beratenen zu erkennen, wie oft die eigenen Grenzen selbst ausgereizt und überschritten werden. Eine klare Sprache verhindert Verschleierungen und benennt Tatsachen (vgl. ebd.). Besteht Inter*-Personen gegenüber eine Unsicherheit in der Ansprache, so ist es empfehlenswert, sie selbst zu fragen, welches Pronomen sie bevorzugen. Berater_innen müssen sich darüber im Klaren sein, dass sie selbst nicht frei von Zuschreibungen und Stigmatisierungen sind, sondern diese verinnerlicht haben und unbewusst reproduzieren. Eine Reflexion und Überprüfung der eigenen Rolle und Haltung muss in regelmäßigen Abständen erfolgen. Als probate Methoden dafür haben sich zum Beispiel Supervision und kollegiale Beratung bewährt. Formen des Austauschs und der Reflexion tragen zur Qualitätssicherung der eigenen Tätigkeit bei.

Dieses Kapitel versteht sich als Zusammenfassung der wichtigsten Empfehlungen hinsichtlich einer gerechten Inter*-Beratung unter pädagogischen, sozialwissenschaftlichen und medizinischen Gesichtspunkten. Diese werden im Folgekapitel erneut aufgegriffen und auf ihre praktische Umsetzung hin abschließend diskutiert.

\section{Pädagogische Standards}

Um Intergeschlechtlichkeit als ernst zu nehmendes Thema in Pädagogik und Sozialer Arbeit zu etablieren, ist eine klare Haltung im Umgang mit ihr notwendig. Übergeordnetes Ziel ist es, gesellschaftskritisch die politische Dimension von Geschlecht zu begreifen. Dasselbe gilt für die Thematisierung des Umgangs mit Intergeschlechtlichkeit in übergeordnetem (gesellschaftlichem) Kontext. Eine angemessene Implementierung, im Sinne eines »Inter*-Mainstreamings «, in die Ausbildungsstandards von Schule, Beruf und Studium ist unerlässlich (vgl. Hechler, 2014, S. 51). Als Anliegen von Inter*-Verbänden steht die generelle Beendigung medizinischer Deutungshoheit klar im Vordergrund. Erst im Anschluss daran rücken Fragen zu Identität in den Fokus (vgl. ebd., S. 49). Die Aussage, dass Identitätsfragen nicht immer an erster Stelle zu verorten sind, wird auch von Lucie Veith gestützt:

»Ich kenne genug intersexuelle Menschen, die sind ganz zufrieden dass sie Männer oder Frauen sind. Die wollen auch nichts anderes hören. Egal, wie ihre Körperlichkeit ist [...]. Die sagen: Ach, dieser ganze Genderkram, der interessiert mich gar nicht. [...] Der andere sagt: Ach, ich glaube ich ändere noch mal eben meinen Personenstand, damit da noch mal politisch was sichtbar wird.[...] Aber das hat eigentlich nichts mehr mit der Identität dann zu tun, sondern eher mit einer politi- 
schen Strategie. Da muss man [...] aufpassen, dass man wirklich erst mal ergründet, auf welcher Basis unterhalten wir uns hier eigentlich. [...] für mich steht der Mensch mit seinen Bedürfnissen eigentlich an erster Stelle (Veith, 2014, S. 4).

Es wird empfohlen, den Einstieg ins Thema nicht über den medizinischen Blick zu nehmen, sondern sich über die Darstellung von individuellen Lebenswelten anzunähern. ${ }^{70}$ Exemplarisch können Inters* als Expert_innen in eigener Sache eingeladen werden, um über persönliche oder übergeordnete Berührungspunkte und Inter*-Lebensrealitäten zu referieren. Eine Reduzierung auf das Merkmal Inter* sollte unterlassen werden. Vielmehr gilt es, dass Inters* $\gg$ als eigenständige Menschen, als Individuen mit eigenen Interessen, Vorlieben, Wünschen, Bedürfnissen etc., die nichts mit ihrem Dasein als Inters* zu tun haben, sichtbar werden « (Hechler, 2014, S. 51). Die Gewalterfahrungen von Inters* sollen transparent gemacht und in den Kontext von Widerstandsbewegungen aus den eigenen Reihen eingebettet werden.

Bearbeitet man das Thema aus einer nicht-intergeschlechtlichen Perspektive, besteht die Gefahr, dass es durch Prozesse des $\gg$ Othering ${ }^{71}{ }^{71} \mathrm{zu}$ einem »Reden-Über « kommt (vgl. ebd., S. 50). Grundsätzlich ist im pädagogischen Handlungsfeld immer von einer Heterogenität in Gruppenkontexten auszugehen, die auch die Anwesenheit von Inters* einschließt. Diesbezüglich sind ein kritisches Bewusstsein und ein sensibler Umgang relevant. Es macht daher auch Sinn, Bündnisse mit anderen Bewegungen herzustellen (vgl. ebd., S. 51), zum Beispiel der LSBTTIQA-Bewegung, der Kinderrechts- oder der anti-kapitalistischen Bewegung.

\section{Sozialwissenschaftliche Standards}

Als Herausgeber_innen eines Sammelbandes zu Erfahrungen und Analysen von Geschlechtszuweisungen positionieren sich Groneberg und Zehnder eindeutig gegen medizinische Interventionen:

70 Pädagogische Materialien für die Bildungs- und Beratungsarbeit sind rar (vgl. Hechler, 2014, S. 50). Einige wenige Schriftstücke und Filmbeiträge wurden bereits im Kapitel zum Forschungsstand und zur aktuellen Debatte vorgestellt.

71 Der Begriff des »Othering « geht auf Gayatri Chakravorty Spivak zurück und bezeichnet den Prozess einer Gruppenzugehörigkeit in Abgrenzung zu einer anderen Gruppe, der klare Attribute, Verhaltensweisen, Ideologien etc. zugeschrieben werden. Es erfolgt eine Aufwertung des Eigenen durch das Abwerten des Anderen (vgl. Spivak, 1985). Julia Reuter spricht in diesem Zusammenhang vom Prozess der »VerAnderung« (vgl. Reuter, 2002). 
»Zum einen ist auf diesem Hintergrund nicht mehr zu verantworten, bei Neugeborenen mit somatosexueller Uneindeutigkeit weiterhin unhinterfragt zur Operation zu schreiten oder zu raten. Zum anderen kann nicht davon ausgegangen werden, dass mit der chirurgischen und hormonellen Geschlechtsanpassung >das Problem < behoben ist. Vielmehr muss zur Kenntnis genommen werden, dass viele Probleme erst durch die Behandlung entstehen « (Groneberg \& Zehnder, 2008, S. 216).

Die Autor_innen fordern, dass eine medizinische Intervention nur bei einer akuten Lebensbedrohung des Kindes vorgenommen werden und das »Fehlen « von vermeintlicher Gesundheit nicht als ausreichend gelten dürfe (vgl. ebd.). Zudem müssen die Interessen des sozialen Beziehungsgefüges, also die des Kindes und der Eltern oder alternativer Familienkonzepte, mit bedacht und berücksichtigt werden. Insofern argumentieren sie für ein Moratorium, wie es auch der Verein Intersexuelle Menschen tut:

$\gg$ Erst mal $[\ldots]$ müssen Schutzräume hergestellt werden, $[\ldots]$ nicht nur [...] im räumlichen, sondern auch in Zeitschienen. Wir brauchen [...] für jedes Kind zum Beispiel ein Moratorium. Und zwar in dem Moment, wo so was auffällt [...] muss klar sein, die nächsten drei Jahre geht da mal gar nichts. Sondern dieses Kind muss jetzt erst mal verstehen, was mit ihm selbst ist, und die Eltern müssen es verstehen, und die Umwelt muss sich darauf einstellen können, und je länger man sich damit beschäftigt, [...] je mehr wächst die [...] Gewissheit, dass alles gut ist, wie es ist « (Veith, 2014, S. 5).

Aus demselben Hintergrund leiten Groneberg und Zehnder (2008) wissenschaftliche Empfehlungen für die transdisziplinäre Arbeit und Auseinandersetzungen um Intergeschlechtlichkeit ab, die nicht zuletzt auch den Bereich der psychosozialen Beratung betreffen und bereichern. Sie legen den Schwerpunkt auf sechs Themenkreise, die im Folgenden skizziert werden: Erziehungsgeschlecht; Diagnostik, Unterstützung und Betreuung; Informationspolitik; chirurgische Eingriffe; Recht sowie Wissenschaft und Forschung.

\section{Erziehungsgeschlecht}

Auch das Anliegen des Deutschen Ethikrates zielte in seinen Grundgedanken darauf ab, aus Analysen Empfehlungen für eine gute Praxis abzuleiten. Michael Wunder sieht in der Möglichkeit eines offenen Erziehungsgeschlechts das Potenzial für einen Paradigmenwechsel: 
$\gg$ Alle Erfahrung zeigt, dass eine bis in Kindheit und Jugend reichende Erziehung, die die Geschlechtseinordnung offen lässt, möglich, wenn auch nicht einfach ist, aber weniger Leid bedeutet als frühzeitig festlegende Operationen, welche die Betroffenen später als traumatisch erleben und von denen ihr Leben gekennzeichnet ist $\ll$ (Wunder, 2012, S. 39).

Groneberg, Werlen und Zehnder stützen Wunders Argumentation in ihren Ausführungen zum Erziehungsgeschlecht und merken an, dass es jede selbst gewählte Geschlechtsidentität anzuerkennen und zu respektieren gilt. Darüber hinaus wird angeraten, dass bei der Wahl des Erziehungsgeschlechts sexuelle Lebensqualität und genitale Sensibilität anzustreben sind (vgl. Groneberg \& Zehnder, 2008, S. 217).

\section{Diagnostik und Betreuung von Beratenen und ihrem Bezugssystem}

Die Komplexität der Relation von Intergeschlechtlichkeit und psychosozialer Beratung nimmt in diesem Punkt einen wichtigen Stellenwert ein. Viele Argumentationsstränge, die in einem der vorherigen Teile dieser Arbeit bereits diskutiert wurden, finden sich hier wieder. Groneberg, Werlen und Zehnder sprechen sich ebenfalls für ein transdisziplinäres Betreuungsteam in einem spezialisierten Zentrum aus. Eine systematische Differenzierung von Sex, Gender und Gender Role, Identity und Desire ist anzustreben. Diese Geschlechtsidentität gilt es im weiteren Verlauf nicht anzuzweifeln. Eltern und Familie sollen in Hinblick auf die damit einhergehenden Herausforderungen durch eine intensive Begleitung durch Fachpersonal unterstützt werden. In der Regel benötigen eher die Eltern als die Kinder Unterstützung:

$\gg$ Ich glaube, $[\ldots]$ dass $[\ldots]$ in den meisten Systemen mit intersexuellem Kind [...] gar nicht [...] das Kind das eigentliche Problem ist, sondern es sind die Eltern. Und [...] das ist auch eine Forderung von uns, wenn Eltern ein Problem haben, dann lasst die Hände weg von den Kindern« (Veith, 2014, S. 14f.).

Auch an diesem Punkt wird auf die Qualität der Selbsthilfe als Unterstützungsund Identifikationsmöglichkeit verwiesen (vgl. Groneberg \& Zehnder, 2008, S. 218). Da der Prozess der Begleitung unter Umständen bis ins Erwachsenenalter andauen kann, wird auch hier die Wichtigkeit einer vertrauensvollen Beratungsbeziehung herausgearbeitet. Unter dem Prinzip psychischer, physischer und emotionaler Unversehrtheit soll ein häufiger Wechsel der Kontakt- und Vertrauensperson nach Möglichkeit unterbunden werden. Zukünftig soll das längst 
überholte Schweigegebot dem Aufklärungsrecht von Kindern »über den eigenen Körper und seine Bedeutung in der Gesellschaft « (ebd.) weichen.

\section{Informationspolitik}

Die Aufklärung des Kindes über die Intergeschlechtlichkeit muss feinfühlig und sorgfältig erfolgen und in die soziale Lebens- und Wertewelt integriert werden, sodass sich anstelle einer Stigmatisierungserfahrung ein Selbstbewusstsein herausbildet. Es gilt auch die Eltern umfassend zu informieren. Im Zusammenhang mit operativen Maßnahmen müssen sie im Vorfeld und ohne Zeitdruck sowohl über Risiken als auch über Nebenwirkungen und Selbstaussagen von »Betroffenen « nach Operationen unterrichtet werden. Ebenso nennenswert bzw. notwendig ist es, über Forderungen von Selbsthilfe und solidarischen Unterstützer_innen nach einem Moratorium aufzuklären und den »Betroffenen « die Option zu eröffnen, auf einen Eingriff gänzlich zu verzichten. Vor allem medizinisches, aber auch soziales und psychologisches Fachpersonal muss (inter*-)spezifisch geschult werden. Eine selbstverständliche Darstellung von Situationen und gesellschaftlichen Bildern erhöht die stabilisierende Wirkung auf »Betroffene «. Deshalb ist es umso wichtiger, daran zu arbeiten, dass die Ansicht eines vielfältigen Geschlechterverständnisses flächendeckend als selbstverständlich anerkannt wird, indem sie beispielsweise Einzug in Bildungspläne und Unterrichtsmaterialien erhält. Dabei ist insbesondere auf eine gendersensible Sprache zu achten und auf diffamierende Bezeichnungen zu verzichten (vgl. Groneberg \& Zehnder, 2008, S. 219).

\section{Medizinische Eingriffe}

Da die meisten Formen intergeschlechtlicher Varianz nicht behandlungsbedürftig sind, stellt sich die generelle Frage nach der Notwendigkeit bzw. der Essenz medizinischer Operationen. Eine Stellvertreter_innen-Entscheidung durch die Eltern ist in allen Fällen kritisch zu hinterfragen und in gravierenden Fällen zu untersagen. Kosmetische Eingriffe können erst im einwilligungsfähigen Alter vorgenommen werden und auch nur dann, wenn die Person selbst sich dafür entscheidet. Auch die Argumentation eines erhöhten Krebsrisikos bei einer NichtEntfernung der Gonaden ist wissenschaftlich umstritten. Bei der Entscheidung für eine Gonadektomie muss über die Konsequenz einer lebenslangen Hormonsubstitution informiert werden. Bei einer Entscheidung gegen einen solchen Eingriff muss auf die Dringlichkeit regelmäßiger Kontrolluntersuchungen verwiesen werden. Generell gilt: Um reflektierte Entscheidungen hinsichtlich jeglicher medizinischer Interventionen treffen zu können, bedarf es einer grundlegenden, 
allumfassenden Aufklärung über Alternativen und Konsequenzen. Viele Eingriffe erfordern lange Nachbehandlungen, deren Ausmaß weder die Eltern noch gar die Kinder abschätzen können. Deshalb ist es umso wichtiger, dass potenzielle Einwilligungs- und Entscheidungsphasen zeitlich offen sind und eine psychosoziale Betreuung innerhalb dieses Zeitrahmens, wenn nicht sogar darüber hinaus, unbedingt gewährleistet ist (vgl. Groneberg \& Zehnder, 2008, S. 220).

Recht

In Bezug auf die rechtlichen Empfehlungen muss auf die Neuregelung des Personenstandsrechts von 2013 als aktuellste gesetzliche Grundlage verwiesen werden. Da Selbsthilfe-Verbände geschlechtszuweisende und geschlechtsvereindeutigende chirurgische Eingriffe unter dem Aspekt einer beeinträchtigten oder zerstörten genitalen Sensibilität gleichsetzen, muss auch in diesem Punkt Forderungen nach rechtlicher Wiedergutmachung bzw. rechtlichen Regelungen nachgekommen werden. Eine Akteneinsicht muss zu jedem Zeitpunkt möglich sein und der Zeitraum der Verjährungsfrist soll gemäß Inter*-Verbänden ausgeweitet werden. Die Erarbeitung von Richtlinien zur Sicherung eines Mindestmaßes an Rechtsansprüchen im transdisziplinären Zusammenschluss wird von den Autor_innen angeraten und gefordert (vgl. Groneberg \& Zehnder, 2008, S. 221).

\section{Wissenschaft und Forschung}

Wissenschaftliche Forschung muss so aufgebaut sein, dass die aus den Resultaten folgenden und daran anschließenden Maßnahmen Inters* gerecht werden. Dies erfordert im Vorfeld eine kritische Betrachtung der eigenen Disziplin unter ethischen Maßstäben, die die Auswirkungen der Forschung auf das subjektive Erleben von Inters* berücksichtigen. Auch in diesem Punkt wird eine interdisziplinäre Zusammensetzung als sinnvoll und erforderlich erachtet (vgl. ebd., S. 221).

\section{Medizinische Standards}

Im Rahmen der Arbeitsgemeinschaft wissenschaftlicher medizinischer Fachgesellschaften (AMWF) entstanden 2007 Leitlinien der Deutschen Gesellschaft für Kinderheilkunde und Jugendmedizin (DGKJ), die sich an medizinisches Fachpersonal richten und Handlungsempfehlungen für Diagnostik und Therapie von intergeschlechtlichen Neugeborenen bereitstellen. Positiv hervorzuheben ist die Zusammenarbeit sowohl mit der XY-Elterngruppe als auch mit dem Verein AGSEltern- und Patienteninitiative, die wertvolle Anregungen beisteuerten. Im Falle 
einer medizinischen Indikation ${ }^{72}$ soll unverzüglich ein diagnostisches Verfahren in Gang gesetzt werden, das Ursache und anschließendes Behandlungsprogramm eruieren und darüber hinaus die Beratung der Familie integrieren soll:

»Die Geschlechtszuordnung soll dabei auf der Grundlage einer Diagnostik und unter Einbeziehung von Experten, nach Möglichkeit in einem Zentrum mit einem erfahrenen multidisziplinären Team [...] und nach offener Darlegung und mit Beteiligung der Eltern und deren Beratern erfolgen « (Holterhus et al., 2007, S. 1).

Im genannten Beratungsteam sind nach Aussage der Autor_innen sowohl Vertreter_innen der Sozialen Arbeit als auch der Ethik inbegriffen (vgl. ebd.). In Anlehnung an die Konsensuskonferenz in Chicago übernimmt die Arbeitsgemeinschaft zwar den daraus hervorgegangen Terminus der »Störungen der Geschlechtsentwicklung «, merkt aber zugleich an, dass dieser von »Betroffenenverbänden « nicht als Selbstbezeichnung angenommen wird (vgl. ebd.). Insgesamt spiegelt die verwendete Sprache jedoch eine stark medizinisch orientierte Perspektive wider. Der diagnostische Prozess soll einerseits der Diagnosestellung dienen, andererseits aber auch der Geschlechtszuweisung. Letzteres erfolgt zwar unter Einbeziehung der Eltern, schließt aber ein offenes Erziehungsgeschlecht gänzlich aus. Als dritter kritischer Punkt wird das Einleiten einer Langzeitbetreuung benannt (vgl. Holterhus et al., 2007, S. 2f.). Positiv ist die Öffnung medizinischen Handelns hinsichtlich sozialer Bezugssysteme, in diesem Fall der Eltern. Jedoch stellt Erziehung kein medizinisches Problem, sondern eine soziale Herausforderung dar, unabhängig davon, ob das Kind intergeschlechtlich geboren wird oder nicht. In dieser Hinsicht ist zu fragen, was genau sich die Arbeitsgemeinschaft unter einer Langzeitbetreuung vorstellt. Ob es sich dabei um die Stabilisierung des zugewiesenen Geschlechts handelt oder um eine Unterstützung der Belange des Kindes und der Familie, wird aus den Leitlinien nicht ersichtlich. Der Prozess der Diagnostik gliedert sich in mehrere Teilschritte: Anamnese, klinische und bildgebende Diagnostik, zytogenetische, hormonelle und molekulargenetische Diagnostik sowie auch weiterführende invasive Diagnostik und Nachweisdiagnostik (vgl. ebd., S. 3f.). Anhand dieser Teilbereiche lässt sich ableiten, dass die Phase der Diagnostik einen langen Zeitraum einnimmt.

Die persönliche Einschätzung von Inters* bezüglich diagnostischer Verfahren entwirft ein konträres Bild zur sachlichen Abhandlung der Leitlinien. Als Impres-

72 Hierzu zählen ausschließlich lebensbedrohliche Formen von Intergeschlechtlichkeit, zum Beispiel hervorgerufen durch Salzverlust oder eine Insuffizienz der Nebenniere. 
sionen werden hier drei biografische Zitate subjektiver Erfahrungen aufgelistet, die jeweils medizinische Grenzüberschreitungen bis hin zu explizit gewalttätigen Handlungen beinhalten:

»Ein 17jähriges Mädchen verbringt ein Vierteljahr im Krankenhaus. Sie wird untersucht, vermessen, fotografiert, operiert, in den gynäkologischen Stuhl gelegt, besichtigt von Studententrupps, die einmarschieren, wenn sie schon zur Inspektion aufgebockt ist. Eine Erfahrung, die viele intersexuelle Kinder und Jugendliche machen: ihre Geschlechtsorgane üben große Faszination aus; das Hinsehen wie das Wegsehen der anderen sind gleichermaßen bedrückend. Für die Seele des Kindes und für sein Trauma aber interessiert sich keiner « (Fröhling, 2003, S. 32).

Nicht alle Menschen, die ein traumatisches Erlebnis hatten, erkranken psychisch daran. Dennoch wird hieraus ersichtlich, wie wenig Beachtung dem emotionalen Empfinden und der Würde der Inters* in diagnostischen Prozessen zukommt. Die grundsätzliche Wahrung der Menschenrechte weicht einer Art ärztlichem Voyeurismus. Die Ablehnung dieses Umgangs muss aus ethischen Gesichtspunkten Inhalt von Handlungsempfehlungen für medizinisches Fachpersonal sein.

$\gg$ Vor kurzem hat sie den Assistenzarzt von damals angerufen. Ein emeritierter Professor inzwischen. Der hat sich sofort erinnert und war ganz aufgeregt. >Sie waren doch mein erster Fall $<$, sagte er und erzählte, daß ihr $>$ Fall $<$ in mehreren medizinischen Artikeln dargestellt ist. Mindestens eine Promotionsarbeit gibt es über Anna. Sie bittet um Zusendung. Er zögert, hat Vorwände. Schließlich rückt er das Buch doch heraus. Sie schlägt es auf. Die Fotos sind bedrückend: ein einsames nacktes Mädchen mit Balken über dem Gesicht. Genitale Details. [...] >Natürlich war die Prozedur damals entwürdigend « « (Fröhling, 2003, S. 37).

Das Zitat verdeutlicht, wie medizinische Diagnostik auf Personen wirkt, die diese über sich ergehen lassen mussten. Leitlinien, die am Wohlergehen des Gegenübers interessiert sind, müssen vorherrschende Umgangsformen kritisch reflektieren und sensibles Vorgehen explizit beinhalten. Auch wenn Holterhus et al. in manchen Punkten ihren Blickwinkel weiten, bleibt die Behandlungspraxis nach wie vor objektivierend.

»Erika beim Gynäkologen. Wegen schmerzhafter Spannungen in der Brust. Versehen mit einem Schreiben, das ihr der Hausarzt im verschlossenen Umschlag in die Hand gedrückt hatte. Der Gynäkologe liest den Brief, sagt: >Sie wissen ja, was 
mit ihnen los ist.< In der Tat, sie wußte es wirklich, vor zwei Wochen hatte sie den Arztbrief geöffnet. >Dann setzen Sie sich mal auf den Stuhl<, sagt der Gynäkologe. Widerstandsunfähig zieht Erika sich aus und setzt sich auf den gynäkologischen Stuhl - eigentlich nicht das richtige Gerät zur Untersuchung von Brustspannungen. Bewegungsunfähig verharrt sie dort, während der Arzt ihr ohne Vorwarnung seinen Finger in die Scheide steckt. [...] Wie in Trance, wie gelähmt verharrt Erika weiterhin auf dem Stuhl. [...] > [S]agen Sie niemandem, was mit ihnen los ist. Das wäre ihr soziales Aus.< Und schließlich fällt ihm noch ein, weshalb sie eigentlich hier ist: $>$ Das mit den Brustspannungen vergeht wieder. $<$ Damit ist sie dann entlassen. $>\mathrm{Ab}-$ artig kalt<, so bleibt ihr diese Begegnung in Erinnerung « (Fröhling, 2003, S. 40).

Obwohl Erika Kasal Beschwerden äußerte, die nicht zwingend mit ihrer_seiner Intergeschlechtlichkeit zu tun haben mussten, wurde er_sie darauf beschränkt. Den Forderungen nach einer transparenten Informationspolitik, wie sie u. a. Groneberg et al. und die Selbsthilfe proklamieren, entspricht solch ein Umgang nicht.

Für ein Therapiekonzept ${ }^{73}$ werden folgende Kriterien als geeignet vorgeschlagen: die Einbeziehung verschiedener fachlicher Disziplinen, einhergehend mit einer umfassenden (medizinischen) Aufklärung der Eltern durch (medizinisches) Fachpersonal. Eltern müssen über die Möglichkeit informiert werden, sich gegen eine Operation zu entscheiden und das Kind in einer offenen Geschlechtskategorie zu erziehen. Es ist daher ratsam, einen Perspektivwechsel vorzunehmen um aus der Rolle des betreffenden Kindes zu argumentieren. Diese Leitlinien versäumen es jedoch, beide Alternativen aufzuzeigen. Diesbezüglich stellt sich die Frage nach der Entscheidungslegitimität der Eltern und nach ihrer Stellvertreter_innenposition im Hinblick auf Maßstäbe der Medizinethik. Allerdings wird darauf verwiesen, dass Operationen mit dem Ziel der chirurgischen Korrektur derzeit kontrovers in der Diskussion stehen. Die Autor_innen beziehen sich dabei auf die gravierenden Resultate der wissenschaftlichen Studien zur Behandlungszufriedenheit (vgl. Holterhus et al., 2007, S. 4f.). Gleichermaßen herrscht Uneinigkeit darüber, ab welchem Alter medizinische Eingriffe für sinnvoll erachtet werden. Während der Beginn einer Hormonersatztherapie erst im Zeitraum der Pubertät angeraten und verortet wird (vgl. ebd., S. 4), gehen die Meinungen bezüglich operativer Eingriffe auseinander. Meistens werden sie innerhalb des ersten Le-

73 Die Bezeichnung »Therapie "hat in diesem Zusammenhang keinen psychologischen Charakter, sondern bezieht sich auf das Behandlungsprogramm, das aufgrund des anatomischen »|st-Zustands", der Diagnose und des prognostizierten Entwicklungsverlaufs von ärztlicher Seite empfohlen wird (vgl. Holterhus et al., 2007, S. 4). 
bensjahres des Kindes veranlasst. In Bezug auf Feminisierungsoperationen heißt es beispielsweise:

»Es wird bei fehlenden medizinischen Komplikationen davon abgeraten, operative Maßnahmen nach dem 12. Lebensmonat bis zur Adoleszenz durchzuführen und insbesondere auf vaginale Dilatationen zu verzichten « (Holterhus et al., 2007, S. 5).

Unter Einbeziehung von Anmerkungen der XY-Elterngruppe wird postuliert, dass »insbesondere die Indikation zur Klitorisreduktionsplastik bei einer Feminisierungsoperation zurückhaltend gestellt werden [sollte], da eine etwas vergrößerte Klitoris der weiblichen Selbstakzeptanz nicht im Wege steht « (ebd.). Dieser Verweis kann auch hinsichtlich der Erhaltung genitaler Sensitivität positiv vermerkt werden. Auch das »Entartungsrisiko « als Argument für die Durchführung einer Gonadektomie wird als nicht wissenschaftlich gesichert angegeben (vgl. ebd.). Dennoch distanziert sich die Arbeitsgemeinschaft in den Ausführungen der Leitlinien von diesen Eingriffen. Zumindest weisen die Autor_innen darauf hin, dass aufgrund des Eingriffs Nachsorgeuntersuchungen bis ins Jugendalter erfolgen müssen, die in einer weiteren Gonadenbiopsie münden (vgl. ebd.). Weiterhin führen sie als Anregung durch die XY-Elterngruppe an:

»Grundsätzlich sollte bei der Indikationsstellung zur Gonadektomie überprüft werden, ob die medizinischen Befunde zulassen, dass die Entscheidungsreife des Kindes abgewartet werden kann « (ebd.).

Alles, was nicht medizinisch indiziert werden kann, wird auch in Zukunft als »psychosozialer Notfall « deklariert. Im Zusammenhang damit werden in den Leitlinien einige wesentliche Punkte benannt (vgl. ebd.):

$>$ Jegliche Entscheidung hinsichtlich medizinischer Maßnahmen muss ohne Zeitdruck und unter Einbeziehung sowohl der Eltern als auch eines interdisziplinären Teams erfolgen.

$>$ Die Einbeziehung der Eltern in die Entscheidungsfindung muss stets und von Anfang an mit bedacht werden.

$>$ Es muss dabei sichergestellt werden, dass die Eltern die Tragweite und die Bedeutung der Maßnahmen verstanden haben.

$>$ Der Verweis auf ein offenes Erziehungsgeschlecht, um »das wahre Wesen des Kindes zu erkennen « (ebd.), wie es die XY-Elterngruppe fordert, erfolgt nur bei einer Unmöglichkeit der Geschlechtszuweisung.

$>$ Die Herstellung von Kontakten zur Selbsthilfe soll ermöglicht werden. 
Um öffentlichen Widerstand gegen medizinische Maßnahmen zu leisten, hat sich bereits 1996 die Arbeitsgruppe gegen Gewalt in der Pädiatrie und Gynäkologie (AGGPG) gegründet, deren vorrangiges Ziel im Verbot invasiver Medizin bei Inters* und in der Erhaltung des ursprünglichen Körpers bestand (vgl. Hechler, 2014, S. 49). Bis heute hat sich diese Forderung auf medizinischer Ebene nicht durchgesetzt. 


\section{Impulse an eine konkrete Umsetzungspraxis}

In diesem letzten Teil der Arbeit geht es insbesondere darum, die aus den Hauptteilen gewonnenen Ergebnisse in Hinsicht auf eine praktikable Umsetzung in der Beratungspraxis zu sichern und Ausblicke für neue Ideen zu entwickeln. Die folgenden fünf Konzeptionsvorschläge haben dabei exemplarischen Charakter und werden als Impulse für anschließende Ausarbeitungen verstanden, zu denen ausdrücklich angeregt werden soll.

Obwohl Intergeschlechtlichkeit nicht als medizinischer, sondern als gesellschaftlicher Handlungsplatz verstanden wird, sind medizinische Denk- und Handlungsweisen nicht losgelöst vom aktuellen Ist-Zustand und seinen existierenden wissenschaftlichen, pädagogischen oder beratenden Strukturen zu begreifen. Es ist sinnvoll, wenn nicht sogar notwendig, an Schauplätzen anzusetzen, die mit Intergeschlechtlichkeit in jedweder Form verknüpft sind. Vorrangiges Ziel dabei ist, den Grundstein für einen kritischen Dialog zu legen, der auf einer diskursiven Auseinandersetzung fußt, um daran anschließend gemeinsam Forderungen der Inter*-Verbände umzusetzen. Die Schnittstelle zwischen Medizin und Beratung wird diesbezüglich als wichtiger Ansatzpunkt angesehen, um neue Handlungsoptionen zu implementieren.

Um speziell sowohl junge Inters* als auch diejenigen zu erreichen, die nicht unmittelbar im Umfeld von inter*-spezifischen Beratungsangeboten leben, ist es ratsam, Online-Ressourcen als professionelle Beratungsangebote anzuerkennen, sie gegebenenfalls zu verifizieren und/oder weiter auszubauen. Daran schließt der Gedanke an, dass herkömmliche Strukturen modifiziert und generell überholt werden müssen. Ein Beratungsangebot im Sinne der klassischen »KommStruktur « muss zugunsten mobiler Formen weichen bzw. durch diese ergänzt werden. 
Nicht alle Inters* suchen Rat und Austausch in spezialisierten Fachstellen. Viele Belange werden in anderen Beratungskontexten (zum Beispiel in FrauenKontakt-Cafés oder innerhalb der Erziehungsberatung) artikuliert. Auch dort müssen Fachpersonen deshalb inter*-spezifisches Grundwissen vorweisen können. Schlussfolgernde Überlegungen zum Ausbau bestehender Strukturen könnten im Sinne eines »Inter*-Mainstreamings « verankert werden. Im letzten Punkt wird die Entwicklung von Kompetenzzentren für Intergeschlechtlichkeit vorgeschlagen, die verschiedene der genannten Optionen unter einem Dach vereinen und aufgrund der »kurzen (Vermittlungs-)Wege « einen entscheidenden Beitrag zu ganzheitlicher und umfassender »Versorgung « und Unterstützung liefern.

\section{Medizin vs. Beratung - eine wichtige Schnittstelle}

Da medizinische Institutionen mehrheitlich die Orte sind, wo Menschen über ihre Intergeschlechtlichkeit in Kenntnis gesetzt werden, kommt ihnen eine wichtige Schnittstellenfunktion zu. Es reicht nicht aus, Konzepte inter*-spezifischer Beratung zu entwickeln, wenn sie nicht da ansetzen, wo der größte Bedarf ist. Der Moment der ärztlichen Diagnosevermittlung wird häufig als Auslöser einer schwerwiegenden Verunsicherung empfunden. Auf die Frage, ob der Prozess der medizinischen Diagnostik häufig psychosoziale Krisen auslöse, antwortet Lucie Veith:

»Genau so ist es, und deswegen können wir die [Medizin/Diagnostik als Ansatzpunkt] gar nicht rauslassen. Oder [...] eine Gewalterfahrung, die in der [...] [medizinischen] Therapie war. Und wie gesagt, es ist immer in Verbindung mit einer Krise. Das heißt, an wen würde ich mich da wenden? An irgendein Krisenzentrum, $[\ldots]$ an irgendeinen $[\ldots .$.$] psychologischen Dienst. Wenn ich an einen$ Selbstmord denke, an wen wende ich mich dann? Da müssen [...] wir uns verknüpfen $\ll$ (Veith, 2014, S. 36).

In jedem Fall ist es notwendig, die Möglichkeit einer psychosozialen Beratungsoption bereitzustellen und nach Bedarf anzubieten. Es reicht dabei nicht aus, Beratung an Selbsthilfestrukturen auszulagern, sondern es bedarf weiterer handlungspraktischer Überlegungen, die dazu beitragen, fachspezifische Inter*Beratungsstrukturen in den Bereich der Medizin zu integrieren und darüber hinaus eine breite, auch an medizinisches Fachpersonal adressierte Aufklärungsarbeit voranzutreiben. Dem Misstrauen und der Skepsis gegenüber medizinischen 
Handlungs- und Denkweisen setzen sogar einige Inter*-Aktivist_innen Gründe für interdisziplinäre und kooperative Alternativen entgegen:

$\gg[$ Ich] verstehe, warum viele von uns auf $>$ Doktor_innen $<$ und all das, wofür sie stehen, mit Angst und Schrecken reagieren. Vergessen wird aber leider viel zu oft, wie sehr wir Verbündete brauchen, vor allem aus der Medizin. Egal, wie sehr wir darauf pochen, dass Ärzt_innen und Chirurg_innen uns zuhören sollten: Die traurige Wahrheit ist, dass sie nur ihren Kolleg_innen zuhören. Anstatt zu polarisieren und diejenigen vor den Kopf zu stoßen, die uns helfen könnten, unsere Ziele zu erreichen, sollten wir Brücken bauen, um medizinisch nicht notwendige Operationen an intergeschlechtlichen Säuglingen abzuschaffen und Räume für die Selbstdefinition und die Anerkennung von Geschlechtern jenseits des binären Systems aufzubauen $\ll$ (Del LaGrace Volcano \& Klöppel, 2013, S. 78).

Die Überlegung einer Implementierung interdisziplinärer Beratungsteams im medizinischen und Gesundheitssektor wird mittlerweile auch durch Aussagen wissenschaftlicher Expert_innen gestützt:

»Der Ethikrat empfiehlt [...], die medizinische Diagnostik und Behandlung von DSD-Betroffenen nur in einem speziell dafür qualifizierten, interdisziplinär zusammengesetzten Kompetenzzentrum von Ärzten, Psychologen, Sozialberatern und anderen Experten vorzunehmen. Die fortlaufende medizinische Betreuung sollte dann in unabhängigen qualifizierten Betreuungsstellen fortgeführt werden, in denen auch nach dem peer-Beratungsprinzip Betroffene andere Betroffene beraten « (Wunder, 2012, S. 40).

Hinsichtlich der Beratungstätigkeit und speziell unter Einbeziehung unterschiedlicher Lebenskontexte beratener Personen proklamiert Nestmann, dass vielfältige Beratungsanforderungen auch vielfältiger Herangehensweisen und Disziplinen mit je spezifischen Ausrichtungen bedürfen, damit ein ganzheitliches und interaktionales Agieren möglich ist:

»In einer Welt immer komplexer werdender Zusammenhänge in allen öffentlichen und privaten Lebenssphären im Großen wie im Kleinen, bei der engen Verschränkung sozialer, psychologischer, bildungs- und berufsbezogener, emotionaler und rationaler, ökonomischer und ökologischer Lebens- und Problemdimensionen im Globalen wie im Lokalen wäre es Hybris, wollte ein und nur ein disziplinärer Zugang die Allzuständigkeit für Beratung für sich reklamieren « (Nestmann, 2008, S. 76). 
Auch Wissenschaft und Forschung stellen Forderungen nach interdisziplinären Kompetenzzentren, um die »Behandlung « fachlich zu bereichern. Konkrete Vorstellungen sind dabei mehr Zeit in der Beratung, weniger Entscheidungsdruck und ein verstärktes Eingehen auf die individuellen Bedürfnisse und Umstände der Personen (vgl. Wunder, 2012, S. 39).

Eine inter- bzw. multidisziplinäre Gestaltung von psychosozialer Inter*-Beratung muss sowohl auf theoretischer als auch auf praktischer Ebene umgesetzt werden und einen empirischen Anspruch (Evaluation, Qualitätssicherung) verfolgen. Beratung verfügt über eine eigenständige Identität und ist aufZukunftsfähigkeit ausgerichtet, indem sie genuine Arbeitsansätze aufgreift und sie aktuellen Anforderungen und Gegebenheiten anpasst. Dabei schreibt Frank Nestmann, dass multidisziplinäre Beratung als Diskussionsentwurf zu betrachten sei, »der aus den Anregungen verschiedener Disziplinen schöpft « (Nestmann, 2008, S. 76f.).

Unter dem Gesichtspunkt möglicher Akteur_innen interdisziplinärer Beratungsteamsäußert Daniela Truffervon Zwischengeschlecht.orgkonkrete Vorschläge:

»Bisher werden Eltern zu 90 Prozent ausschließlich von Endokrinologen und Kinderchirurgen beraten und betreut. Werden überhaupt Psychologen und Sozialpädagogen hinzugezogen, so spielen sie im > multiprofessionellen Team $<$ höchstens eine Nebenrolle. Wir fordern, dass stattdessen spezialisierte Psychologen und Sozialpädagogen Ansprech- und Kontaktpersonen für die Eltern sein sollen. Mediziner sollen nur für medizinisch notwendige Behandlungen zugezogen werden. Für den berühmten > psychosozialen Notfall< der Eltern braucht es kein Skalpell am Kind, sondern psychologische und sozialpädagogische Betreuung für die Eltern, und gegebenenfalls später auch für die betroffenen Kinder und Jugendlichen selbst « (Truffer, 2011, S. 2).

Im Ausbildungskatalog medizinischer Berufe kommen Einheiten zu psychosozialer Beratungstätigkeit selten explizit, meistens nur randständig vor. So kommt es nicht zuletzt zu beruflichen Überforderungsmomenten, wenn ein adäquater Umgang mit Menschen, die sich in akuten Krisensituationen befinden, gefordert ist. Andreas Hechler verweist darauf, sich in einem ersten Schritt nicht davon verunsichern zu lassen:

»Es ist, das sei hier ausdrücklich gesagt, vollkommen in Ordnung, unsicher zu sein und Wissenslücken zu haben. Dies kann als Herausforderung begriffen werden, damit einen für sich produktiven Umgang zu finden, Wissenslücken zu schließen 
und Selbstsicherheit bezüglich des Themas Intergeschlechtlichkeit zu gewinnen « (Hechler, 2014, S. 48).

Kathrin Zehnder, der_die Erfahrungsberichte von Mitgliedern der Selbsthilfegruppe XY-Frauen inhaltlich analysierte, kommt zu dem Schluss, dass vor allem die Soziale Arbeit als involvierte Disziplin in der Lage ist, Inters* bei ihrer individuellen Problembewältigung zu unterstützen, zu begleiten und je nach Wunsch zu vertreten. Sozialarbeiter_innen können dabei eine Schnittstellenfunktion übernehmen, deren Aufgabe es ist, die verschiedenen Sichtweisen und (professionellen) Haltungen anderer Disziplinen zu bündeln, um gemeinsam im interdisziplinären Team Perspektiven im Sinne intergeschlechtlicher Personen zu entwickeln und aufzuzeigen (vgl. Zehnder, 2008, S. 25). Beratungsteams unterschiedlicher Disziplinen verknüpfen Wissensbestände verschiedener Fachrichtungen miteinander. Die wichtigsten dieser interdisziplinären Komponenten für die Beratung im Kontext von Intergeschlechtlichkeit sind: Inter*-Selbsthilfe, Soziale Arbeit, Sexualwissenschaft, Psychologie/Psychotherapie, Traumapädagogik, Medizin und Recht. Diese interagieren in gleichberechtigter Kooperation und erfassen das Handlungsspektrum von Beratung in ganzheitlicher und effektiver Weise (vgl. Sickendiek et al., 1999, S. 221f.). Interdisziplinäres Vorgehen bedeutet aber auch immer eine Gratwanderung zwischen eigener Abgrenzung und Kooperation mit anderen Handlungsformen und Akteur_innen, die ebenfalls zum unterstützenden Netzwerk gehören. Dazu ist es nötig, den Blick über die Grenzen der eigenen Disziplin hinaus zu wagen und so zur Professionalisierung der eigenen Arbeit beizutragen (vgl. ebd., S. 225f.). Dies gilt im Kontext von Intergeschlechtlichkeit insbesondere für das medizinische Handlungsfeld.

Diese allgemeingültigen Ziele werden durch die Forderung nach medizinischer und gesundheitlicher Selbstbestimmung von Inters* ergänzt. Sie erfordern die grundsätzliche Wahrung der körperlichen Unversehrtheit sowie die Entpathologisierung von sexueller und geschlechtlicher Diversität in gleichem Maße (vgl. Lenz et al., 2012, S. 64f.). Solange ein grundsätzliches Verbot aller geschlechtszuweisenden und -vereindeutigenden Maßnahmen an Inters* vor dem Zeitpunkt ihrer selbstbestimmten Einwilligung sowie die flächendeckende Installation interdisziplinärer Arbeitsbündnisse noch nicht durchgesetzt sind, ist eine medizinische Selbstverpflichtung zur generellen Weitervermittlung an psychosoziale Beratungsstrukturen dringend notwendig, bevor medizinische Interventionen in Betracht gezogen werden. Diese Maßnahme würde für Familien und Inters* gewährleisten, sich nach der Diagnosemitteilung zeitlich unbefristet einen Überblick sowohl über medizinische Handlungsmöglichkeiten als auch über 
Möglichkeiten des eigenen Umgangs im Hinblick auf ihr persönliches Wohlbefinden zu verschaffen. Zur praktischen Umsetzung bieten sich hier verschiedene Optionen an, die unter Umständen in Relation miteinander treten können: eine Verknüpfung mit Inter*-Netzwerkstrukturen, die Etablierung von interdisziplinären Arbeits- und Aktionsbündnissen, das Erstellen und Auslegen inter*spezifischer Beratungsflyer in medizinischen Einrichtungen und Wartezimmern sowie die Entwicklung einer Broschüre als Handreichung, die das vorhandene inter*-spezifische Wissen bündelt, die wichtigsten bundesweiten Informationen ${ }^{74}$ und Anlaufstellen zum Thema Inter* beinhaltet und somit medizinischen Instanzen zur Orientierung und Weitervermittlung dient.

Abschließend werden im Folgenden die wichtigsten Anregungen zum medizinischen Umgang mit Intergeschlechtlichkeit zusammenfassend festgehalten. Eine ausreichende und umfassende Aufklärung und Information hinsichtlich aller Stationen der Diagnostik, operativen Wegen und der Hormonbehandlung gegenüber Eltern und Inters* ist in jedem Fall zu gewährleisten. Daran anschließend dürfen diese Eingriffe nur nach umfassender Aufklärung und mit ausdrücklich informierter Einwilligung der betreffenden Inters* und ihrer Eltern umgesetzt werden. Transparenz hinsichtlich der Diagnose (Besonderheiten der Diagnose, Folgen und Risiken von Eingriffen, Aufzeigen von Perspektiven und alternativen Behandlungsmethoden) nimmt diesbezüglich einen hohen Stellenwert ein. Auch Kinder haben ein Recht, über ihre Intergeschlechtlichkeit informiert und aufgeklärt zu werden. Dies ist in jedem Fall zu berücksichtigen und muss altersgemäß durchgeführt werden. Die Möglichkeit zur Wahl eines offenen Erziehungsgeschlechts ist ausdrücklich zu benennen. Jegliche Kommunikation im Kontext medizinischer Information und Mitteilung bedarf einer angemessenen Sprache (Verzicht auf medizinischen Fachjargon, »barrierefreie « Sprache) sowie einer fachlich-professionellen Übermittlung der Diagnose. Im Anschluss daran sind umgehend Angebote psychosozialer Unterstützung und fachspezifischer Begleitung zu unterbreiten. Dies bezieht sich in gleichem Maße auf das Herstellen von Kontakt- und Austauschmöglichkeiten, um »Peer-Support « zu ermöglichen. Als übergeordnetes Ziel gilt es, grundlegend einen Paradigmenwechsel hinsichtlich der »Optimal Gender Policy« anzustreben und die Entwicklung neuer Behandlungsrichtlinien voranzutreiben.

74 Innerhalb der Inter*-Community wird oft beklagt, dass es an empfehlenswerten Therapeut_innen und Ärzt_innen (darunter insbesondere Endokrinolog_innen) mangelt. Eine bundesweite Recherche nach Empfehlungen von Fachpersonen, die Inters* nicht pathologisierend gegenüberstehen, könnte eine Möglichkeit darstellen, Inters* schmerzvolle Erlebnisse im Vorfeld zu ersparen. 


\section{Einstiegspforte: Onlineplattform}

Der Kontakt und die Vernetzung zu anderen Inters* wird von der Inter*-Community selbst als »enorm wichtiger, stärkender und hilfreicher Selbstermächtigungsprozess « beschrieben, hinsichtlich dessen das Internet eine besondere Bedeutung habe (vgl. Hechler, 2014, S. 53). Online-Ressourcen sind durch ihre Lebensnähe in der Inter*-Community als Medium sehr beliebt. So schreibt beispielsweise AiChih Chiu zu der Webseite der »Organisation Intersex International « (OII):

»Die OII-Webseite hat mir sehr dabei geholfen, etwas über mich als inter* Person zu lernen. Sie gab mir das Gefühl, dass ich endlich einen Platz und Raum in der Welt hatte, in dem ich mich verorten konnte (Chiu, 2013, S. 59).

Beratungsstellen mit langem Vorlauf und bürokratischen Anmeldeprozeduren - insbesondere jene, die mit Anrufbeantwortern arbeiten - wirken oft abschreckend (vgl. Vogt, 2004, S. 215) und verstärken die innere Hemmschwelle, sich Beratungsangeboten zu öffnen. Der Zugang zu neuen Medien kann sich demnach als niedrigschwellige Einstiegspforte auszeichnen.

Online ${ }^{75}$, Chat- oder Telefonberatungen beschreiben Formen der Beratung unter Einbeziehung verschiedener (technischer) Medien. Es handelt sich dabei um sprachliche (Telefonat), schriftliche (E-Mail, Chat, Forum) oder visuelle (Skype, Videochat) Kommunikationsformen. Der Großteil der genannten Möglichkeiten ist internetbasiert ${ }^{76}$. Im Gegensatz zu Face-to-Face-Beratungen finden die Onlineberatungen über eine größere Distanz statt, »bei der sich Berater/in und Klient/in vielleicht sehen und hören (Videochat), aber nicht berühren und riechen können « (vgl. Engelhardt \& Storch, 2013, S. 4).

Mit dem Fokus auf Intergeschlechtlichkeit und Beratung steht Lucie Veith, selbst in Selbsthilfe und Fortbildung von Multiplikator_innen tätig, neuen medialen Beratungsformen kritisch gegenüber. Auf die Frage, wie er_sie die Implementierung einer bundesweiten Onlineplattform bewerte, antwortet Veith:

75 Es handelt sich hierbei nicht um Anfragen, die per Kontaktformular gesendet werden. Diese Möglichkeiten dienen lediglich der Kontaktaufnahme und implizieren keinen beratenden Prozess. Sie entsprechen auch nicht den gesetzlichen Datenschutzbestimmungen, da zum Erhalt einer Rückantwort immer eine E-Mail-Adresse angegeben werden muss. Wenn also im Folgenden die Rede von Onlineberatung ist, wird diese hier genannte Option außer Acht gelassen (vgl. Engelhardt \& Storch, 2013, S. 3).

76 »Online« bezeichnet in diesem Zusammenhang die aktive Verbindung mit dem Internet (vgl. Engelhardt \& Storch, 2013, S. 4). 
$\gg[$ Also] Chat-Beratung [...] geht gar nicht, Telefonberatung unter Umständen, aber [...] du siehst [...] dein Gegenüber nicht. [Mit] Chat-Beratung haben wir ganz schlechte Erfahrung [gemacht], wir haben ja [...] eine Onlineberatung, eine anonyme, über Spira-Net, und das machen wir ja schon seit zehn Jahren, und da ist die Erfahrung, da machen wir das über E-Mail-Verkehr. Das heißt, die Menschen wenden sich anonym an uns, sie wissen, mit wem sie sprechen, aber wir wissen nicht, mit wem wir verkehren, und wir versuchen dann natürlich, [...] diese Anonymität erst mal zu wahren [...], und dann bieten wir aber auch sehr schnell [...] an, um den Zugriff von anderen Beratern wegzunehmen, schreib mich doch direkt an. Meine Email-Adresse ist: lucie@xy-frauen oder [...] lucie@intersexuelle-menschen.net. Damit ist [...] dieses ganz enge Persönliche natürlich dann geschmiedet. Und diese [...] persönliche Beziehung ist dann auch aufgebaut. Wir vereinbaren dann auch immer relativ früh [...] ein Telefonat miteinander. Um die Stimme mal gehört zu haben. Für viele intersexuelle Menschen ist es ein Riesen-Erlebnis, auf der anderen Seite der Leitung irgendeine Stimme zu hören, die dann auch empathisch [klingt]. Und das ist echt spannend, [...] ich mache das nämlich auch immer so, dass ich dann in diesem Telefonat sogar ein persönliches Treffen vereinbare, entweder fahre ich dann dorthin oder wir treffen uns, wenn das nicht so weit weg ist, irgendwo auf der Hälfte oder so oder nehmen uns gemeinsam irgendwas vor. [Die] Menschen sind bereit, dafür zu reisen, aber eben nicht permanent, sondern einmal. Und eine Beratung [...] lebt nicht von einer Einmaligkeit. Aber ein Treffen zu organisieren, einfach hinzufahren das erste Mal und dann weiterhin über Telefon das zu machen, das wäre zum Beispiel eine Möglichkeit. Über Telefonberatung. So wie es die Seelsorge macht. Telefonseelsorge. Das funktioniert auch. Also, da muss man noch mal besondere Techniken haben, aber [...] das geht. Also ich halte das [für] besser, [...] vielleicht ist es heute mit dem Chat, das kann man versuchen, das kann man sicherlich anbieten und auch parallel anbieten, aber Telefon [...] und Chat, also da würde ich eher ein Skype-Gespräch [anbieten], [...] so ein Video-Telefonat, das könnte zukunftsweisend sein. Dass man eben [...] den anderen auch sieht, wenn er spricht « (Veith, 2014, S. 34f.).

Die Vermutung liegt nahe, dass vor allem Jugendliche Online-Angebote nutzen, weil diese angrenzend an ihre Lebenswelt einen ersten Eindruck vermitteln, wie sich Beratung anfühlt. Sie bieten zunächst die Möglichkeit einer unverbindlichen Anfrage. Nestmann et al. bestätigen die These, dass das Internet als Quelle der Kontaktaufnahme zu Beratung genutzt wird, es bietet einen niedrigschwelligen Zugang an und verzeichnet steigende Zuwachsraten (vgl. Sanders, 2004, S. 799). 
Lucie Veith spricht sich für verbindlichere Angebote aus, die, wenn auch nach anfänglicher Distanz, in einen Beratungsprozess außerhalb virtueller Settings übergehen:

$\gg[$ So] was kann man doch [...] über eine SMS [...] machen. [...] Dann hast du zumindest schon mal eine Telefonnummer. Das machen wir ja. [Eine] Erstkontaktaufnahme kann auch über SMS passieren « (Veith, 2014, S. 35).

Anhand der Interviewaussagen wird deutlich, dass Lucie Veith sehr daran gelegen ist, im Bereich der spezifischen Inter*-Beratung direkte Kontakte herzustellen und Inters* an feste Strukturen anzubinden. Er_sie legt Wert auf eine persönliche und vor allem visuelle Begegnung.

Engelhardt und Storch geben ebenfalls an, dass »Onlineberatung im psychosozialen Bereich [...] vor allem auf die textbasierten Formen der Kommunikation über das Internet begrenzt « sei (Engelhardt \& Storch, 2013, S. 2). Schriftliche Sprache an sich kann aus unterschiedlichen Gründen ausschließend wirken - ein Faktor, der in Bezug auf textgebundene Kommunikations- und Beratungsformen nicht ganz unerheblich ist. Denn zunächst müssen Menschen, um diese Angebote in Anspruch zu nehmen, lesen und schreiben können. Darüber hinaus kann die Form der schriftsprachlichen Formulierung des eigenen Anliegens als hochschwellig empfunden werden und überfordern. Oft ist es zu Beginn eines Prozesses den Hilfesuchenden selbst noch gar nicht möglich, den konkreten Auftrag zu formulieren. Die Verbalisierung bzw. Verschriftlichung eines klaren Beratungsauftrags setzt die Erwartung voraus, dass bereits eine Reflexion über die eigene Problematik stattgefunden haben muss, um das Anliegen klar umreißen zu können (vgl. ebd., S. 5). In Face-to-Face-Beratungssituationen dient das Erstgespräch dieser Auftragsklärung und wird bereits als Teil eines möglichen Beratungsprozesses $^{77}$ angesehen. Engelhardt und Storch fragen diesbezüglich, »ob wir uns in der psychosozialen Onlineberatung von der Vorstellung rein textbasierter Kommunikation lösen müssen, wenn wir zielgruppenorientiert arbeiten möchten « (ebd., S. 7). Die Aussage über mögliche Nachteile bezüglich des Schreibprozesses kann jedoch ebenso positiv ausgelegt werden. So sehen Engelhardt und Storch den wesentlichen Vorteil von Onlineberatung im Moment der dafür notwendigen

77 Nicht alle Erstgespräche münden automatisch in einen längerfristigen Beratungsprozess. Es kann sein, dass durch das Erstgespräch bereits Anliegen geklärt wurden und es bei einer einmaligen Sitzung bleibt. Es kann aber auch sein, dass aus unterschiedlichen persönlichen, formalen oder inhaltlichen Gründen gegen die Aufnahme einer weiterführenden Beratung entschieden wird. 
Selbstreflexion, die »im Schreibprozess beim Ratsuchenden in Gang gesetzt wird und einen sehr wesentlichen Anteil des Beratungsprozesses ausmacht, da schon der Schreibvorgang an sich zu Ordnung und Klärung beiträgt « (vgl. ebd., S. 4). Auch Sickendiek, Engel und Nestmann erkennen ein positives Potenzial der Onlineberatung - nämlich hinsichtlich einer Professionalisierung von Beratung im Allgemeinen. Aus der Betrachtung einer internationalen Perspektive auf Zugang und Nutzen neuer Medien kritisieren die Autor_innen, dass diese zuweilen soziale Ungleichheiten zwar verfestigen, allerdings als Medium entkontextualisierter Information und Kommunikation die generellen Möglichkeiten von Beratungen ergänzen. Mediengestützte Netzwerke dienen oft als erste Informationsquelle und bieten schnelle, anonyme und dadurch unverbindliche Austauschmöglichkeiten an. Im Zeitalter der digital natives ${ }^{78}$ bleiben Face-to-Face-Beratungen zwar auch weiterhin zentrale Stützen zukünftiger Beratung, jedoch eignen sich Online-Angebote vor allem, um Einstiegsbarrieren und Hemmschwellen zu senken (vgl. Sickendiek et al., 1999, S. 225). Darüber hinaus weisen zusätzliche webbasierte Angebote in hohem Maße auf einen konkreten Lebensweltbezug hin. Sie erreichen - vor allem orientiert an jungen Menschen - dadurch zudem eine breitere Zielgruppe derer, die nicht in Großstadtnähe leben:

»[Es] ist auf jeden Fall für viele Leute eine geringere Hemmschwelle. Ich glaube, viel tatsächlich, was es bis jetzt so an Peer-to-Peer-Beratung gibt, gerade in dem Bereich, passiert zurzeit auch online. Und deswegen glaube ich halt auf jeden Fall, dass es halt irgendwie Sinn macht, das auch online [...] anzubieten, ja. Gerade halt einfach/[wenn] man dann an Leute denkt quasi, die jetzt zum Beispiel nicht so städtisch oder so auch wohnen, ist das, glaube ich, total sinnvoll « (Kampagne Dritte Option, 2014b, S. 29).

Neben der Gefahr einer möglichen Verzerrung der Situation durch technische Probleme in Bild und Ton (vgl. Engelhardt \& Storch, 2013, S. 3) erfordert die Inanspruchnahme webbasierter Beratungsleistungen Kenntnisse im Umgang mit diesen Medien sowie der Handhabung der Geräte. Im Hinblick auf diejenigen, die nicht im Zeitalter des Internets aufgewachsen sind, bedeutet dies unter Umständen einen Nachteil, diese Form der Beratung nicht (ohne Hilfe) für sich nutzen zu können. Das steht im Gegensatz zur bundesweiten Erreichbarkeit auch

78 Der Terminus digital native wurde durch den US-amerikanischen Pädagogen Mark Prensky geprägt. Er beschreibt diejenige Generation, die mit digitalen Technologien und dem Internet aufgewachsen ist (vgl. Prensky, 2001). 
für jene, die älter und weniger mobil sind. Darüber hinaus schließt es sowohl bundesweit als auch international generell diejenigen Menschen aus, die über keinen Internetzugang verfügen.

Eine weitere Überlegung ist, dass textungebundene, audiovisuelle Kommunikationsformen (Videochat, Skype-Telefonat) von jenen Ratsuchenden abgelehnt werden, denen der Faktor der Anonymität besonders wichtig erscheint. Da es durchaus eine Hemmschwelle darstellen kann, sich direkt als Person in einer Anlauf- und Beratungsstelle als Hilfesuchende_r vorzustellen, erscheinen Onlineberatungen im ersten Schritt attraktiv, weil sie eine gewisse Form der Anonymität vermitteln. Das benennt auch der_die Vertreter_in der Kampagne Dritte Option entsprechend:

»Dazu fällt mir ein, dass es natürlich Situationen geben kann, wo ich halt irgendwie vielleicht Probleme habe, die ich halt alleine nicht so ganz lösen kann, und dass es dann irgendwie gut ist, wenn ich irgendwo hingehen kann, um Unterstützung zu bekommen. Und trotzdem sehe ich natürlich auch, dass es ganz schnell so ein ganz komisches Verhältnis werden kann, also im Endeffekt ist es ja so ein bisschen, ich glaube, es für mich selber auch zum Beispiel schwierig ist oder schwierig wäre manchmal zu einer Beratungsstelle zu gehen, weil ich das Gefühl habe, damit mache ich mich so klein irgendwie, und auch Angst davor habe quasi, dass so eine Machtposition quasi, die die andere Person dadurch unterhält, dass ich dahin komme und halt irgendwie sage, ich hab irgendwie ein Problem, dass das halt dann schwierig ist, und gerade weil wir natürlich auch in einer Gesellschaft leben, die halt immer eigentlich erwartet, dass man funktioniert « (Kampagne Dritte Option, 2014b, S. 3).

Gerade im Hinblick auf anfängliche Unsicherheiten in Bezug auf Beratungen, werden Face-to-Face-Beratungen im Erstkontakt eventuell als zu bedrohlich erlebt. Visuelle Kombinationen kommen demnach den Formen klassischer Präsenzberatung zu nahe. Online-Angebote können aber auch zur bewussten Inszenierung seitens der Ratsuchenden genutzt werden. Diesbezüglich sieht Lucie Veith die Gefahr einer Instrumentalisierung solcher Angebote:

$»[$ Dir] geht ja was verloren, $[\ldots]$ wenn du nur mit ihm telefonierst oder $[\ldots]$ wenn du nur E-Mails hast. Weißt du, man muss ja auch immer gucken, bis zu welchem Grad mache ich da jetzt noch mit und ab wann sage ich: >So, jetzt komm! Jetzt drehen wir uns zum dritten Mal im gleichen Kreis $<$, und der andere weiß es auch und spielt mit mir (Veith, 2014, S. 35). 
Im Gegenzug dazu betonen Engelhardt und Storch, dass auch Präsenzberatung nicht frei von Inszenierung ist (vgl. Engelhardt \& Storch, 2013, S. 7).

Festzuhalten gilt hinsichtlich aller Befürchtungen und Überzeugungen: Es ist nur dann die Rede von expliziter psychosozialer Onlineberatung, wenn es sich tatsächlich um zwischenmenschliche Interaktionen von mindestens zwei Personen handelt, die gemeinsam an der Bewältigung geäußerter Schwierigkeiten arbeiten (vgl. Döring, 2003, zit. n. Engelhardt \& Storch, 2013, S. 8). Frank Nestmann empfiehlt eine Verknüpfung digitaler und analoger Beratungsformate (vgl. Nestmann, 2008, zit. n. Engelhardt \& Storch, 2013, S. 9) - je nach Anliegen, Setting und subjektiven Vorlieben. Hierfür sprechen auch persönliche Aussagen »Betroffener «, die einen ersten Zugang zu Beratung und eine mediale Begleitung zwar in Erwägung ziehen, diese Form nach dem Aufbau einer gewissen Vertrauensbasis aber nicht mehr als ausreichend empfinden:

» $[$ Also] ich glaube schon, dass ich jetzt zum Beispiel so ein Mensch wäre, ich würde auch da wieder sagen, mir würde das halt auch nicht reichen, also es gibt dann so einen bestimmten Bereich, wo es mir dann sogar fast, also ich glaube, es ist/das Positive an so Internet ist halt wie gesagt, dass ich da so Wissen sammeln kann und dass man sich selber nicht so, ja, nicht so viel von sich zeigen muss so schnell, trotzdem glaube ich, dass ich [über] einen gewissen Punkt auch nicht hinauskommen würde quasi, wenn das halt nur im Internet war, wenn man dann irgendwann doch/wenn es um bestimmte/an bestimmte Persönlichkeitsbereiche ranzukommen, dann doch vielleicht irgendwie mehr Kontakt/mehr an Nähe/oder ich zumindest, einen Menschen auch sehen müsste« (Kampagne Dritte Option, 2014b, S. 29).

Hinsichtlich der voranschreitenden Ausweitung potenzieller neuer Beratungsformate und vielfältiger Beratungsszenarien werden Berater_innen zahlreiche zusätzliche Kompetenzen abverlangt, die es in der Aus- und Fortbildung zu berücksichtigen und die es in diese zu integrieren gilt (vgl. Engelhardt \& Storch, 2013, S. 10) - ein Punkt, der sich auch explizit in den Lehrplänen der Ausbildungsinstitute niederschlagen muss.

\section{Das Rad nicht ständig neu erfinden: Ausbau bestehender Strukturen}

Ein sichtbarer Zuwachs an Fachberatungsangeboten lässt in der Regel auf eine öffentliche, politische Akzeptanz und Anerkennung der betreffenden Thematik 
schließen (vgl. Reutlinger, 2004, S. 1197). Im Kontext von Intergeschlechtlichkeit spiegelt es ein Wissen über gesellschaftliche und strukturelle Zusammenhänge von Gewalterfahrungen wider. Ein Ausbau inter*-spezifischer Beratungsangebote spricht demzufolge für eine steigende Sensibilität hinsichtlich dieses Themenund Beratungsfeldes. Dem stehen Arbeitsfelder und Beratungsbereiche gegenüber, die nicht ausdrücklich auf inter*-spezifische Themen ausgerichtet sind. Vor allem im Bereich der Jugendhilfe sind große Handlungsunsicherheiten zu verzeichnen. Diese »Berührungsängste « nicht als Herausforderung anzunehmen, also ein Nicht-Umgang mit Schwierigkeiten und Überforderungen, kann nicht zuletzt den Verlust beruflicher Professionalität bedeuten. Dem kann zukünftig durch die Aufnahme praktischer Fragen der Geschlechterspezifik und Genderkompetenz in Ausbildungsstandards verschiedener sozialer, pädagogischer, gesundheitlicher, sozialrechtlicher und therapeutischer Berufe entgegengewirkt werden. Im Sinne eines »Inter*-Mainstreamings « würden Ausbildungsstandards kompakte Inter*-Fachinformationen beinhalten und über intergeschlechtliche Lebensweisen aufklären. Nur durch eine Vermittlung von und durch Einblicke in die Lebenswirklichkeiten von Inters* können Perspektivwechsel überhaupt erst ermöglicht werden. Im Rahmen dessen würden begleitende und unterstützende Strukturen im Umgang mit Intergeschlechtlichkeit gemeinsam entwickelt und/oder vorgestellt (vgl. Kompetenzzentrum geschlechtergerechte Kinder- und Jugendhilfe Sachsen-Anhalt e. V., 2014). Spezifische Beratungskompetenzen werden durch Aus- und Weiterbildungen, berufsbegleitend durch Erfahrungen mit Arbeitsprozessen oder durch Erkenntnisse des kollegialen Austauschs erworben (vgl. Schiersmann \& Thiel, 2004, S. 901). Nicht nur im Aus- und Weiterbildungsbereich, an Berufsschulen oder in Form von Multiplikator_innen-Schulungen, sondern auch im Bildungssektor muss Inter* fester Bestandteil schulischer und sexueller Bildung und in den Curricula der Länder fest verankert werden. Es ist wichtig, eine Sichtbarkeit dessen zu schaffen, was durch eine Unsichtbarkeit bzw. ein »Unsichtbar-Machen « keinen Einzug in den öffentlichen und wissenschaftlichen Diskurs erhält. Gabriele Dietzke beschreibt diese Handlungsweise als Gegenstrategie zum sogenannten vanishing act:

»Über das Sichtbarmachen von Intersexualität könnten dabei sowohl systematische Leerstellen von Gender Studies wie auch Gründe für die strukturelle Gewaltsamkeit gegenüber intersexuellen Menschen erhellt werden « (Dietzke, 2006, S. 47).

Derselbe Ansatz begründet ein Inter*-Counseling. Zwar sind zur unterstützenden Beratung von spezifischen Inter*-Bewältigungsproblemen keine völlig neuen 
professionellen Ansätze notwendig, dennoch können bestimmte Problembereiche durch Aspekte spezifischen Vorgehens unterstützt werden. Demnach siedelt sich psychosoziale Beratung im Kontext von Intergeschlechtlichkeit da an, wo sie über einen reinen Informationscharakter hinausgeht, und endet dort, wo eine Ressourcenorientierung nicht ausreicht, um der Lebensbewältigung gerecht zu werden, und das Format ein verbindlicheres Setting mit klarer Rahmenstruktur im Sinne einer Psychotherapie benötigt.

Darüber hinaus existieren innerhalb der Beratungslandschaft bereits vielfältige Angebote, die sich den Themen Geschlecht und Sexualität widmen. Dadurch könnte der Eindruck entstehen, dass das Themenfeld Intergeschlechtlichkeit innerhalb bestehender Beratungsangebote ausreichend abgedeckt sei. Lucie Veith entgegnet dieser Annahme, dass der Ausbau bestehender Strukturen und die fachspezifische Schulung von Multiplikator_innen nicht unkritisch zu betrachten ist:

$\gg[$ Dann] stoßen wir ganz schnell an [unsere] Grenzen, wenn es [...] nicht wirklich anspruchsvoll und [...] gut geschult [ist]. [Die] musst du auch in Sozialrecht schulen, [...] wie lange soll so eine Spezialausbildung dauern? Wie [...] groß ist der Bedarf und wie viele Leute willst du ausbilden? [...] [Was] ich mir vorstellen könnte [ist, dass es] am Ministerium [...] eine Plattform gibt. Das [man es an] einem großen Gesundheitsamt [...] unterbringt, anhängt. Denn ich sehe noch mal eine ganz andere Gefahr [...], ich weiß nicht ob ich als betroffener Mensch an eine Sexualberatung gegangen wäre. Glaube ich nicht. Weil, Sexualität war nicht das Problem « (Veith, 2014, S. 35).

Lucie Veith spricht sich zwar für ein Inter*-Mainstreaming aus, empfiehlt jedoch nicht, alle bereits existierenden Beratungsstellen hinsichtlich einer Inter*-Fachspezifik auszubauen:

» [Ich] bin auch dafür, dass alle Bescheid wissen müssen, dass es so etwas [wie Intergeschlechtlichkeit] gibt. [...] und [...] die müssen [...] diese Schlüsselworte kennen, wenn [Inters* in die Beratung] kommen. Die [...] meisten Menschen kommen mit einer Diagnose. Die kommen nicht mit: > Mit mir ist was komisch $<\ll(e b d$.$) .$

Veith sieht beispielsweise sowohl die »einzelne ProFamilia-Einrichtung für geeignet « an (ebd., S. 25) als auch »Familien-Beratungsstellen, die [...] einen Sonderdienst anbieten « (ebd., S. 36).

Somit lässt sich zusammenfassend festhalten: Es ist durchaus gewünscht, dass Beratungsstellen über Grundkenntnisse in Bezug auf Intergeschlechtlichkeit 
und damit in Relation stehende Weitervermittlungskompetenzen verfügen. Gleichermaßen werden spezialisierte Fachfortbildungen für sinnvoll erachtet. Diese werden vor allem auch im Bereich der Kinder- und Jugendarbeit empfohlen. Dennoch überschreitet die Komplexität des Themas eine simple Integrierung in fachverwandte Beratungsstellen, wenn sie professionell und fachspezifisch umgesetzt werden soll, wie es bereits Lucie Veith in einem der vorherigen Zitate zur Begründung anmerkte. Demnach sind die genannten Ansätze als Ergänzungen zu zusätzlichen inter*-spezifischen Fachberatungsstellen zu verstehen, die das Thema Inter* als Teil geschlechtlicher und identitärer Vielfalt sichtbar und erfahrbar machen. Inter*-spezifische Konzeptionierungen verfügen über ein größeres Potenzial, Angebote zu schaffen, die von Inters* anerkannt und genutzt werden, weil sie sich an ihren konkreten Lebensrealitäten und Bedürfnissen orientieren und dadurch der Komplexität der Thematik im Ganzen gerecht werden.

\section{Mobile Beratung: Ein neuer Weg des Zugangs bzw. eine Umkehr der Hemmschwelle}

Die sich mittlerweile etablierenden Beratungsformen, die durch mehr Flexibilität und offenere Settings gekennzeichnet sind, stellen erhöhte Anforderungen an das Beratungsprofil der handelnden Akteur_innen. Agieren Berater_innen im freien Feld sozialer Begegnungen, sei es durch aufsuchende oder mobile Beratungsoptionen, so stellt dies auf den ersten Blick einen Verlust dar. Bewährte formalisierte Rahmenbedingungen, darunter zum Beispiel sichernde Beratungsrituale, ein festes Setting und/oder professionelle Distanz zwischen den Akteur_innen, werden dahingehend verunmöglicht. Andererseits bedeutet es auch den Gewinn, sich im Sinne einer System-Umwelt-Beziehung unvoreingenommen auf die jeweilige Situation einzulassen, ohne ein vorgefertigtes Pauschalangebot abzuspielen, das einer immer gleichen Logik und starren Struktur folgt (vgl. Thiersch, 2004b, S. 706).

Eine gute Erreichbarkeit von Beratungsangeboten ist weitestgehend auf den großstädtischen Raum begrenzt. Angesichts dessen stellt sich die Frage nach einer flächendeckenden Versorgung. Somit stehen Ideen zum Ausbau von Außenstellen bzw. mobilen Angeboten in der Überlegung (vgl. Schiersmann \& Thiel, 2004b, S. 904). Lucie Veith kritisiert das vorherrschende Angebot von inter*-spezifischer Beratung und favorisiert die mobile Beratung mit entsprechender Begründung:

$\gg$ [Das aktuelle bundesweite Beratungsangebot] ist wirklich grauenhaft. [...] Mit ganz $[\ldots]$ wenigen Ausnahmen $[\ldots]$. Es gibt $[\ldots]$ wenige wirklich praxisorientierte 
Menschen, [die anderen sind so] verkopft, so [...] lebensfern, das muss ja auch immer noch zum Lebensentwurf [...] der einzelnen Menschen passen. Wie gesagt, diese Modelle, wie sie im Moment laufen, dass sie eben hauptsächlich in den Großstädten sind, [...] aber die meisten Menschen in der Fläche leben, [...] und wenn dann auch noch die Dichte der zu Beratenen relativ gering ist, dann kann es eigentlich nur eine Lösung geben, nämlich dieses Mobile. Der mobile Service sozusagen, [...] die mobile Beratung. Und weil sie so hoch qualifiziert sein muss und so anspruchsvoll ist, [...] kann, glaube ich, auch kein Berater, der normalerweise am Tag so vier Beratungen oder fünf Beratungen macht, auch nur eine Beratung am Tag leisten. Und [...]ich glaube, auch für die Beraterqualität wird es gut sein, dass er die Möglichkeit hat, da noch mal drei Stunden hinzufahren und drei Stunden zurückzufahren, um auch diese Zeit der Reflexion wiederzugeben für sich selbst. Denn man [muss] aus diesen Beratungsgesprächen auch immer wieder [...] unbeschadet raus gehen « (Veith, 2014, S. 24).

Beratung findet in traditionellem Rahmen an einem konkreten Ort, gewöhnlich in einer Beratungseinrichtung, statt und verfolgt eine klare Zeit- und Vorgehensstruktur, beispielsweise durch das wiederkehrende und Sicherheit vermittelnde Eingangsritual. Moderne Vorstellungen setzen auch hinsichtlich des Settings und der Beratungsstruktur auf flexiblere Konzepte, die sich an der konkreten Lebenswelt der Ratsuchenden orientieren und sich näher an ihrem realen Umfeld bewegen (vgl. Sickendiek et al., 1999, S. 223). Die klassische »Komm-Struktur « wird hinsichtlich größerer Mobilität der Beratenden durch » aufsuchende « Angebote überwunden. Das bedeutet konkret, Angebote dort anzusiedeln, wo sich die adressierte Zielgruppe aufhält (z. B. Netzwerke, Familien, Jugendzentren) und wo Beratung mit anderen Diensten verknüpft wird und nicht nur in der Rolle der Zuträger_in von Beratungssituationen verbleibt (vgl. ebd.). Das gewährleistet nicht nur eine niedrigere Hemmschwelle, sondern auch eine Neuorientierung von Beratungskonstellationen. Die Möglichkeit einer mobilen Beratung hat auch präventiven Charakter. Dennoch muss Beratung, wie bislang durchgeführt und nicht zuletzt auch finanziert, neu strukturiert werden. In Anlehnung an Lucie Veith werden sich Beratungsstrukturen verändern. Anstatt täglich eine Abfolge mehrerer Beratungen hintereinander zu leisten, wird es im Anschluss an installierte mobile Beratungsformate, je nach Anfahrtsweg, nur noch möglich sein, sich auf maximal eine Beratungssituation pro Tag einzustellen. Der Vorteil liegt dabei einerseits in einer sorgfältigen Vorbereitungszeit (durch die Anfahrt) und andererseits in einer direkt anschließenden Reflexion sowohl der Beratungssituation als auch der eigenen Rolle auf der Rückfahrt. Zu Beginn kann es sinnvoll sein, 
einzelne Angebote pro Bundesland zu etablieren, die anfänglich hohe Anfahrtswege einberechnen müssen. Sobald erste Evaluationsresultate erhoben sind, wird sich zeigen, ob sich diese Idee zukünftig durchsetzen kann und flächendeckend aufgebaut wird. Dadurch würden sich wiederum die Anfahrtswege verkürzen und somit müssen Vor- und Nachbereitung explizit mit in die Konzeption eingearbeitet werden. Die mobile Form der Beratung ist zeitlich nicht festgelegt bzw. nicht einheitlich eingrenzbar. Die beratene Person und der Umfang des Beratungsauftrages legen den zeitlichen Aktionsrahmen in Zusammenarbeit mit der beratenden Person fest.

Inter*-spezifische Beratung heißt immer auch, neue Ideen zu entwickeln und Optionen in Betracht zu ziehen, die bislang zum Beispiel an ökonomischen Hürden gescheitert sind, weil sie oberflächlich betrachtet im Gegensatz zur herkömmlichen täglichen Beratungsfrequenz zu wenig effektiv erschienen. Allerdings bedeutet eine Aktualitätsüberprüfung des Status quo von Beratungshandeln auch immer einen Willen zur Neuorientierung bzw. Weiterentwicklung. Diese neuen Visionen potenzieren sowohl den Qualitätsstandard der eigenen Arbeit als auch die Effizienz beraterischen Handelns insgesamt.

\section{„Alles unter einem Dach« - Die Zukunft liegt im Aufbau von Kompetenzzentren}

Anknüpfend an die vorigen Überlegungen dieser Arbeit und unter dem Aspekt der Grundzüge einer gerechten Inter*-Beratung, haben sich einige ernst zu nehmende Ideen entwickelt, an die es auch im folgenden Punkt anzuknüpfen gilt. Es hat sich gezeigt, welche Wichtigkeit den Aspekten Vertrauen und Sicherheit im Beratungsprozess zukommt und wie viele Inters* aus Angst vor entwürdigenden und nicht wertschätzenden Erfahrungen an hochschwelligen Beratungszugängen scheitern. Dem steht ein vertrauensvoller Umgang auf Augenhöhe gegenüber. $\mathrm{Zu}$ gewährleisten ist eine gut funktionierende Vernetzungs- und Vermittlungsarbeit, wenn die Denk- und Arbeitsweisen der empfohlenen Spezialist_innen bekannt sind und als professionell bewertet werden. Eindrücke und Einschätzungen formen sich vor allem durch prozesshafte und langfristige interdisziplinäre Zusammenarbeit sowie kollegialen Austausch und werden durch »kurze Wege « begünstigt. Dies erfordert klare Unterstützungsleistungen seitens des Beratungspersonals. So bietet es sich beispielsweise bei der Vermittlung eines_r Psychotherapeut_in an, anzumerken, dass man ihre_seine Arbeit als empfehlenswert einschätzt und gegebenenfalls aus beruflicher Erfahrung bestätigen kann, dass die 
Person Inters* vorurteilsfrei und akzeptierend gegenübersteht. Unter Umständen ist der_die Psychotherapeut_in sogar Mitglied des Beratungsteams. Zusätzlich kann das gemeinsame Vereinbaren von Erstterminen, ebenso wie das Angebot einer Begleitung zum ersten Treffen, als hilfreich erlebt werden. Schließlich gelangt man zu dem Punkt, dass »kurze Wege « nicht nur im Sinne von Kontaktpflege und Vernetzung zu denken sind, sondern auch räumlich. Befinden sich verschiedene Akteur_innen und Disziplinen eines Inter*-Kompetenzteams am selben Ort, sprich im selben Gebäude, verringern sich Hemmschwellen zunehmend und wird vor allem eine schnelle und effektive Weitervermittlung unterstützt.

Der Aufbau fachspezifischer Kompetenzzentren für Intergeschlechtlichkeit stellt die wohl weitreichendste Form der konkreten Umsetzungspraxis dar. Verschiedene am Beratungsprozess beteiligte Instanzen und Professionen könnten sich unter einem Dach vereinen, »kurze Wege « zwischen den unterschiedlichen Fachdisziplinen ermöglichen, Übergänge gestalten und begleiten sowie eine ertragreichere Kooperationsarbeit gewährleisten. Sie würden dazu beitragen, Prozesse zu intensivieren und das ganzheitliche Erfassen individueller Lebenslagen zu optimieren. Bereits in der Online-Studie des Deutschen Ethikrates wurde deutlich erkennbar, dass mehr als drei Viertel aller Befragten, konkret 86 Prozent, außerklinische Kontakt- und Beratungszentren ausdrücklich befürworten (vgl. Wunder, 2012, S. 39).

Denkbar wäre zudem die Entwicklung von Beratungsteams, sozusagen einer dualen Form des Beratungsgespräches. Dabei kommt es allerdings weniger auf den Ort als auf die Art der Vernetzung an. Wie in Bezug auf die übergreifende Zusammenarbeit der verschiedenen Fachdisziplinen, so sind auch hier sowohl die Frage nach finanziellen Mitteln für den Aufbau von Inter*-Kompetenzzentren als auch die hohe Verantwortlichkeit gegenüber den Beratenen zu bedenken:

$»[\mathrm{Da}]$ ist oft nicht viel Geld, [...] also muss man auch dort vielleicht versuchen, [sich] gleich mit öffentlichen Stellen eng zu verknüpfen, hoheitliche Aufgaben mit zu übernehmen sozusagen aus [dem Gesundheitsbereich],[...] oder vielleicht sogar an ein Ministerium [...] hängen, [...] um eben auch eine Kontinuität zu wahren, das ist ja auch wichtig, das kann nämlich nicht eine Geschichte [...] sein, [...] die nur projektbezogen für drei Jahre oder so gesichert ist, sondern die muss länger gesichert sein. [Wenn] du heute einen Zehnjährigen übernimmst [...], musst du den ja unter Umständen zumindest bis 20 [begleiten], zumindest [...] musst du der Ansprechpartner bleiben. Wenn das nicht gesichert ist, kann man so was eigentlich nicht aufziehen. Und wenn du das nicht selbst leisten kannst, dann musst du zumindest eine zweite oder dritte Person dann rechtzeitig schon mit reinziehen, [...] ich glau- 
be auch, dass [...] eine ganz enge Verknüpfung [...] mit der Selbsthilfe ganz wichtig ist. Da sehe ich auch zum Beispiel einen Teil der Möglichkeiten, Klienten zu [...] bekommen. [...] [Dort], wo die Selbsthilfen wirklich an ihre Grenzen stoßen, dass man dort auf eine professionalisierte Beratungsstruktur zurückgreifen kann. An die man dann verweist « (Veith, 2014, S. 33).

Selbsthilfe- und Elterngruppen könnten sich mit eigenen Gruppenräumen ansiedeln und durch ihre Präsenz stärker in allgemeine Prozesse eingebunden werden. Tagungsräume für Fachveranstaltungen und Weiterbildungsangebote, allgemeine Beratungsräume und/oder auch ein Kontaktcafé sind weitere Optionen, die einen konzeptionellen Aufbau von Kompetenzzentren begründen und begünstigen. 


\section{Ausblick}

Die Resultate der zentralen medizinischen Studien zur Lebensrealität intergeschlechtlicher Menschen haben deutlich gemacht, dass die medizinische Behandlungspraxis gravierende Auswirkungen auf das psychische und physische Wohlbefinden intergeschlechtlicher Menschen hat und nicht zuletzt in hohem Maße die Lebensqualität einschränkt. Diesbezüglich haben die Resultate einen deutlichen Bedarf an fachspezifischer Beratung unter psychosozialen Aspekten aufgezeigt. Die vorgestellten Selbsthilfeorganisationen übernehmen dabei einen großen und wichtigen Anteil grundlegender Strukturen und werden im Sinne des Peer-Ansatzes als wichtige Instanz für eine vertrauensvolle und selbstermächtigende Basisarbeit angesehen. Sie geben einen Überblick über aktuelle Debatten innerhalb der Inter*-Community im deutschsprachigen Raum und verleihen dem Thema Intergeschlechtlichkeit darüber hinaus durch ihren öffentlichen Aktionismus und politischen Aktivismus gesellschaftliche Transparenz.

Die Interviews mit Expert_innen vom Verein Intersexuelle Menschen und der Kampagne Dritte Option bestätigen die Vorannahmen und Aussagen der Studien im Hinblick auf Beratungsbedarf und spezifizierte Inter*-Angebote. Eine breite Bündnisarbeit und interdisziplinäre Vernetzung sind zugunsten einer kooperativen Zusammenarbeit mit professionalisierten Beratungsinstanzen sowie formalisierten Ausrichtungen von Beratung speziell für Inters* ausdrücklich erwünscht und gewinnen zunehmend an Bedeutung. Die besondere Berücksichtigung der »Betroffenenperspektive « und der Forderungen der Inter*-Verbände sind richtungsweisend für die Entwicklung inter*-spezifischer Beratungskriterien. Die beratungsrelevanten Kernpunkte orientieren sich sowohl am Konzept des Peer-Involvements als auch an empowernden Ansätzen. Basisaufgabe von Inter*Beratung ist die Wiederherstellung von Autonomie der Lebenspraxis, die einen 
damit verbundenen Emanzipationsanspruch impliziert. Somit ist Inter*-Beratung immer auch eine emanzipatorische Beratung, die ein ganzheitliches Begreifen des Geschehens auf der Basis einer Subjekt-Subjekt-Interaktion gewährleisten muss.

Darüber hinaus ist sie fachlich psychosozial ausgerichtet und verfügt über dezidierte Beratungsqualifikationen. Aufgrund einer besonderen Sensibilität gegenüber Inters* im Beratungsprozess werden Ansätze vorgeschlagen, die zwar auf bewährte Strukturen zurückgreifen, diese aber mit neuen Entwicklungen bedürfnis- und lebensweltorientiert zu verbinden versuchen. Eine Beratung auf Augenhöhe, die durch professionelle Nähe gekennzeichnet ist, hat das Potenzial, langfristig Prozesse effektiv und professionell zu begleiten. Obwohl auch im medizinischen Sektor mehrheitlich die Rede von verbesserter Aufklärung ist und der Schwerpunkt neuerer Entwicklungslinien auf Beratungsstandards liegt, muss Inter*-Beratung die Haltung einer kritisch reflektierten Menschenrechtspolitik vorantreiben, die eine grundlegende Pathologisierung und Medikalisierung von Geschlecht ablehnt.

Intergeschlechtlichkeit als komplexes Phänomen erfordert komplexe Beratungsstrukturen. Strukturelle Bedingungen können durch die Implementierung verschiedener spezifischer Impulse angeregt und umgesetzt werden. Insgesamt sind die Präferenzen im Bereich Inter* stark an heuristischen Vorgehensweisen im Beratungsprozess orientiert (vgl. Schiersmann \& Thiel, 2004, S. 900). Ein übergreifendes Konzept von Inter*-Mainstreaming und Inter*-Counseling würde Grundkenntnisse und Handlungsfähigkeiten in bestehenden Beratungs- und Bildungsbereichen sicherstellen und geeignete Weitervermittlungsqualifikationen gewährleisten. Verschiedene Zusatzoptionen können Beratungsgrundlagen für intergeschlechtliche Menschen spezialisieren und professionalisieren. Ohne Anspruch auf Vollständigkeit verstehen sich die genannten Impulse interdisziplinärer Beratungsteams, eines Ausbaus des Online-Sektors und der Beratungsmobilität, eines Sicherstellens von Grundlagenwissen zu Inter* in allen fachverwandten Beratungs- und Bildungsstrukturen sowie des Aufbaus von Inter*-Kompetenzzentren als Ansatzpunkte aktueller diskursiver Auseinandersetzungen um Intergeschlechtlichkeit. Demnach kann sich diese Publikation nur als Zwischenbilanz verstehen - eine Analyse des Status quo mit einigen Überlegungen, die darüber hinausragen, denn:

»Wir sind noch weit entfernt von Anerkennung oder Gleichberechtigung für Zwitter und einer Normalität, in der ein Herm selbstverständlich die medizinische Versorgung erhält, die er_sie benötigt und will, statt der bisherigen normierenden und meist ohne aufgeklärte Einwilligung zuteil gewordenen $>$ Fürsorge $<$. Noch sind 
es Ausnahmen, in denen junge Herms so aufwachsen können, wie sie in die Welt gekommen sind, ihnen Zeit gegeben wird, herauszufinden, wie sie sich in dieser Welt identifizieren möchten und wie sie dabei ihre Körperlichkeit erleben « (Kromminga, 2013, S. 98).

Es ist daher unabdingbar, Bipolarität aufzubrechen und pathologisierende und normalisierende Handhabungsversuche zu überwinden, um die Akzeptanz geschlechtlicher Vielfalt voranzutreiben und Inters* als Menschen mit individuellen Interessen, Vorlieben, Erfahrungen und Lebensrealitäten wahrzunehmen. 


\section{Abkürzungsverzeichnis}

AGS Adrenogenitales Syndrom

AMWF Arbeitsgemeinschaft wissenschaftlicher medizinischer Fachgesellschaften

BMBF Bundesministerium für Bildung und Forschung

CAT Committee against Torture

CEDAW Convention on the Elimination of all Forms of Discrimination

DGKJ Deutsche Gesellschaft für Kinderheilkunde und Jugendmedizin

DSD Disorders/Differences of Sex Development

DSM-IV Diagnostischer und Statistischer Leitfaden zu psychischen Störungen

e.V. eingetragener Verein

FGM Female Genital Mutilation

ICD-10 Die Internationale statistische Klassifikation der Krankheiten und verwandter Gesundheitsprobleme

IMeV Intersexuelle Menschen e.V.

ISNA Intersex Society of North America

IVIM Internationalen Vereinigung Intergeschlechtlicher Menschen

LSBTTIQA Lesbisch, schwul, bisexuell, trans*/transident, transsexuell, inter*, queer, asexuell

OII Organisation Intersex International

ORQO Oriental Queer Organisation Austria

SHG Selbsthilfegruppe

SMS Short Message Service

UKE Universitätsklinikum Hamburg-Eppendorf

UN United Nations

VIMÖ Verein Intersexueller Menschen Österreich 


\section{Literatur und Quellen}

Anna (2001) http://xy-frauen.de/geschichten/anna.php (Zugriff: 18.07.2014).

Antonovsky, A. (1997). Salutogenese. Zur Entmystifizierung der Gesundheit. Tübingen: dgvt.

Appel, E. (2002): Auswirkungen eines Peer-Education-Programms auf Multiplikatoren und Adressaten - eine Evaluationsstudie. Freie Universität Berlin. http://www.diss.fu-berlin. de/diss/servlets/MCRFileNodeServlet/FUDISS_derivate_000000000567/05_kapitel04. pdf?hosts= (Zugriff: 02.08.2014).

Arbeitsgruppe Ethik im Netzwerk Intersexualität »Besonderheiten der Geschlechtsentwicklung « (2008). Ethische Grundsätze und Empfehlungen bei DSD. Therapeutischer Umgang mit Besonderheiten der Geschlechtsentwicklung/Intersexualität bei Kindern und Jugendlichen. Monatsschrift Kinderheilkunde, 153(8), 241-245.

Backes, H. (2003). Peer Education. In Bundeszentrale für gesundheitliche Aufklärung (Hrsg.), Leitbegriffe der Gesundheitsförderung. Glossar zu Konzepten, Strategien und Methoden in der Gesundheitsförderung (S. 176-179). Schwabenheim a. d. Selz: Verlag Peter Sabo.

Backes, H. (2004). Peer Education. E\&C Zielgruppenkonferenz: Zivilgesellschaft stärken - Bürgerschaftliches Engagement in E\&C-Gebieten fördern. In Regiestelle E\&C der Stiftung SPI \& Sozialpädagogisches Institut Berlin (Hrsg.), Zivilgesellschaft stärken - Bürgerschaftliches Engagement in E\&C-Gebieten fördern. Dokumentation zur Veranstaltung vom 3. und 4. November 2004. (S. 19-24). Berlin: Bundesministerium für Familie, Senioren, Frauen und Jugend.

Bamberger, G. G. (2004). Beratung unter lösungsorientierter Perspektive. In F. Nestmann, F. Engel \& U. Sickendiek (Hrsg.), Das Handbuch der Beratung. Band 2. Ansätze, Methoden und Felder (S. 737-748). Tübingen: dgvt.

Barth, E., Böttger, B., Ghattas, D. C. \& Schneider, I. (Hrsg.). (2013). Inter. Erfahrungen intergeschlechtlicher Menschen in der Welt der zwei Geschlechter. Berlin: NoNo.

Bauer, M. \& Zwischengeschlecht.org (2014). Dokumentation: Intersex-Genitalverstümmelungen (IGM) in Baden-Württemberg. http://stop.genitalverstuemmelung.org/public/Doku_ IGM_Intersex_BW_2014_web.pdf (Zugriff: 07.07.2014).

Baumgartinger, P.P. (2008): Lieb schtean Les schtean, schtean du das gerade liest ... Von Emanzipation und Pathologisierung, Ermächtigung und Sprachveränderungen. liminalis. Die Zeitschrift für geschlechtliche Emanzipation und Widerstand, 2008/2, 24-39. 
Berendsen, E. (2013). Mein Freund Gerda. In Frankfurter Allgemeine Zeitung (FAZ) vom 20. Oktober 2013, Nr. 142. S. 59.

Berliner Zentrum für Selbstbestimmtes Leben behinderter Menschen e.V. (2014): Peer-Counseling. http://www.bzsl.de/peer_counseling.html (Zugriff: 02.08.2014).

Bongard, N. (о.J.). Junge oder Mädchen? Oder beides? http://www.stern.de/gesundheit/ intersexualitaet-junge-oder-maedchen-oder-beides-1529064.html (Zugriff: 30.03.2014).

Bora, A. (2012). Zur Situation intersexueller Menschen. Bericht über die Online-Umfrage des Deutschen Ethikrates. Berlin: Eigenverlag.

Borkenhagen, A. \& Brähler, E. (Hrsg.). (2014). Schwerpunktthema: Intersexualitäten. Psychosozial $37(1)$.

Bornstein, K. (2014). Mein-Geschlecht.de. http://www.meingeschlecht.de/mein-geschlecht/ (Zugriff: 13.08.2014).

Brunner, F. Prochnow, C., Schweizer, K. \& Richter-Appelt, H. (2012). Hängt das Geschlecht vom Körper ab? Körper- und Geschlechtserleben bei Personen mit kompletter Androgeninsensitivität (CAIS). In K. Schweizer \& H. Richter-Appelt (Hrsg.), Intersexualität kontrovers. Grundlagen, Erfahrungen, Positionen (S. 225-252). Gießen: Psychosozial-Verlag.

Budde, J. \& Venth, A. (2010). Genderkompetenz fürlebenslanges Lernen. Bildungsprozesse geschlechterorientiert gestalten. Bielefeld: W. Bertelsmann.

Butler, J. (1991). Das Unbehagen der Geschlechter. Frankfurt a. M.: Suhrkamp.

Butler, J. (1995). Körper von Gewicht. Die diskursiven Grenzen des Geschlechts. Frankfurt a. M.: Suhrkamp.

Chase, C. (1995). Beleidigende Vernunft. http://blog.zwischengeschlecht.info/pages/Cheryl -Chase-Beleidigende-Vernunft-Affronting-Reason-1995 (Zugriff: 22.05.2014).

Chiu, A. (2013). Liebe in Aktion. In E. Barth, B. Böttger, D. C. Ghattas \& I. Schneider (Hrsg.), Inter. Erfahrungen intergeschlechtlicher Menschen in der Welt der zwei Geschlechter (S. 59-68). Berlin: NoNo.

Del LaGrace Volcano \& Klöppel, U. (2013). Activists and Allies. Ein Gespräch. In E. Barth, B. Böttger, D. C. Ghattas \& I. Schneider, Ina (Hrsg.), Inter. Erfahrungen intergeschlechtlicher Menschen in der Welt der zwei Geschlechter (S. 73-78). Berlin: NoNo.

Deutscher Bundestag (2013). Beschlussempfehlung und Bericht des Innenausschusses (4. Ausschuss) zu dem Gesetzesentwurf der Bundesregierung. Drucksache 17/10489. Entwurf eines Gesetzes zur Änderung personenstandsrechtlicher Vorschriften (Personenstandsrechts-Änderungsgesetz - PStRÄndG). http://dip21.bundestag.de/dip21/btd/17/ 121/1712192.pdf (Zugriff: 12.08.2014).

Deutscher Ethikrat (2012a). Intersexualität. Stellungnahme. Berlin: Eigenverlag.

Deutscher Ethikrat (2012b). Deutscher Ethikrat: Intersexuelle Menschen anerkennen, unterstützen und vor gesellschaftlicher Diskriminierung schützen. Pressemitteilung 01/2012 vom 23. Februar 2012. http://www.ethikrat.org/presse/pressemitteilungen/2012/ pressemitteilung-01-2012 (Zugriff: 02.12.2014).

Dietzke, G. (2006). Schnittpunkte. Gender Studies und Hermaphroditismus. In G. Dietzke \& S. Hark (Hrsg.), Gender kontrovers. Genealogien und Grenzen einer Kategorie (S. 46-68). Königstein/Taunus: Ulrike Helmer.

Dombrowe, B. J. (2010). Tabu Intersexualitaet - Menschen zwischen den Geschlechtern. https:// www.youtube.com/watch?v=rNg8NhVwb5s (Zugriff: 19.08.2014).

Döring, N. (2003). Sozialpsychologie des Internet. Göttingen: Hogrefe Verlag.

Dörrlamm, M. (2006). Professionelle Nähe - auf Distanz zum Status Quo. Widersprüche. Zeitschrift für sozialistische Politik im Bildungs-, Gesundheits- und Sozialbereich, 26(100), 155-160. 
Engel, F. (2002). Beratung im Zeitalter ihrer technischen Reproduzierbarkeit. In F. Nestmann \& F. Engel (Hrsg.), Die Zukunft der Beratung (S. 135-154). Tübingen: dgtv.

Engel, F., Nestmann, F. \& Sickendiek, U. (Hrsg.). (2004). »Beratung « - Ein Selbstverständnis in Bewegung. In F. Nestmann, F. Engel \& U. Sickendiek (Hrsg.), Das Handbuch der Beratung. Band 1: Disziplinen und Zugänge (S. 33-44). Tübingen: dgvt.

Engelhardt, E.M. \& Storch, S.D. (2013). Was ist Onlineberatung? - Versuch einer systematischen begrifflichen Einordnung der »Beratung im Internet«. e-beratungsjournal.net. Fachzeitschrift für Onlineberatung und computervermittelte Kommunikation, 2. http://www. e-beratungsjournal.net/ausgabe_0213/engelhardt_storch.pdf (Zugriff: 18.08.2014).

Eugenides, J. (2003). Middlesex. Roman. Reinbek bei Hamburg: Rowohlt.

Eveline (2008). Erfahrungen einer Betroffenen. In M. Groneberg \& K. Zehnder (Hrsg.), »Intersex«. Geschlechtsanpassung zum Wohle des Kindes? Erfahrungen und Analysen (S. 19-24). Freiburg: Academic Press.

Frankfurter Allgemeine Zeitung.net (2008). Intersexualität. Chirurg muss für Entfernung von Geschlechtsorganen zahlen. http://www.faz.net/aktuell/gesellschaft/gesundheit/ intersexualtaet-chirurg-muss-fuer-entfernung-von-geschlechtsorganen-zahlen-1515845. html (Zugriff: 12.02.2015).

Fröhling, U. (2003). Leben zwischen den Geschlechtern. Intersexualität - Erfahrungen in einem Tabubereich. Berlin: Ch. Links.

Ghattas, D.C. (2013). Menschenrechte zwischen den Geschlechtern. http://www.intersexualite. de/index.php/vorstudie-menschenrechte-zwischen-geschlechtern/ (Zugriff: 13.08.2014).

Grawe, K., Donati, R. \& Bernauer, F. (1995). Psychotherapie im Wandel. Von der Konfession zur Profession. Göttingen: Hogrefe Verlag.

Groneberg, M. \& Zehnder, K. (Hrsg.). (2008). »Intersex«. Geschlechtsanpassung zum Wohle des Kindes? Erfahrungen und Analysen. Freiburg: Academic Press.

Hamburger Forschergruppe Intersex (2008). http://www.intersex-forschung.de/ (Zugriff: 19.08.2014).

Hart, P. (2013). Orchideen züchten. Eine inter* Erfahrung auf Film. In E. Barth, B. Böttger, D.C. Ghattas \& I. Schneider (Hrsg.), Inter. Erfahrungen intergeschlechtlicher Menschen in der Welt der zwei Geschlechter (S. 79-85). Berlin: NoNo.

Hartmann, J. (2004). Dynamisierung in der Triade Geschlecht - Sexualität - Lebensform: Dekonstruktive Perspektiven und alltägliches Veränderungshandeln in der Pädagogik. In S. Timmermanns, E. Tuider \&. U. Sielert (Hrsg.), Sexualpädagogik weiter denken. Postmoderne Entgrenzungen und pädagogische Orientierungsversuche (S. 59-77). Weinheim/München: Juventa.

Hechler, A. (2012). Intergeschlechtlichkeit als Thema geschlechterreflektierender Pädagogik. In Dissens e. V., K. Debus, B. Könnecke, K. Schwerma \& O. Stuve (Hrsg.), Geschlechterreflektierte Arbeit mit Jungen an der Schule. Texte zu Pädagogik und Fortbildung rund um Jungen, Geschlecht und Bildung (S. 125-136). Berlin: Hinkelsteindruck.

Hechler, A. (2014). Intergeschlechtlichkeit als Thema in Pädagogik und Sozialer Arbeit. Sozialmagazin. Die Zeitschrift für Soziale Arbeit, 39(3-4), 46-53.

Herrmann, S. K. (2003). Performing the Gap - Queere Gestalten und geschlechtliche Aneignung. arranca! Aneignung I, 28, 22-26. (Wiederabdruck in A. G. Gender-Killer (Hrsg.). (2007). Das gute Leben. Linke Perspektiven auf einen besseren Alltag (S. 195-203.). Münster:unrast.)

Holterhus, P.-M., Köhler, B., Korsch, E. \& Richter-Unruh, A. (2007). Störungen der Geschlechtsentwicklung. In Leitlinien der Gesellschaft für Kinderheilkunde und Jugendmedizin. Arbeitsgemeinschaft Pädiatrische Endokrinologie (APE) als Sektion der Deutschen Gesellschaft für Kinderheilkunde und Jugendmedizin (DGKJ) sowie der Deutschen 
Gesellschaft für Endokrinologie (DGE) Arbeitsgruppe Disorders of Sex Development (DSD) der APE. http://www.awmf.org/uploads/tx_szleitlinien/027-022I_S1_Stoerungen_ der_Geschlechtsentwicklung_2010-10.pdf (Zugriff: 08.08.2014).

Hörmann, G. (2004). Gesundheitswissenschaft/Medizin und Beratung. In F. Nestmann, F. Engel \& U. Sickendiek (Hrsg.), Das Handbuch der Beratung. Band 1: Disziplinen und Zugänge (S. 171-180). Tübingen: dgvt.

Humer, R. (2014). Blog Zwischi. http://www.zwischi.blogspot.de/(Zugriff: 02.07.2014).

Intersex Society of North America (o. J.). http://www.isna.org/ (Zugriff: 04.07.2014).

Intersexuelle Menschen e.V. (2009). Lila, oder was ist Intersexualität? http://intersexuelle -menschen.net/pdf/Lila.pdf (Zugriff: 13.08.2014).

IntersexuelleMenschen e.V.(2010). http://forum.intersexuelle-menschen.net/ (Zugriff:05.07.2014). Intersexuelle Menschen e.V. (2011). Flyer Intersexuelle Menschen e.V. http://db.intersexuelle -menschen.net/includes/pdf/Flyer_IMEV.pdf (Zugriff: 12.08.2014).

Intersexuelle Menschen e.V (o.J.a). Forderungen. http://www.intersexuelle-menschen.net/ forderungen/(Zugriff: 07.07.2014).

Intersexuelle Menschen e.V (o.J.b). Selbsthilfegruppen Intersexuelle Menschen. http://www. intersexuelle-menschen.net/switch-shg.php (Zugriff: 08.07.2014).

Intersexuelle Menschen e.V (o.J.c). Selbsthilfegruppen der XY-Frauen. http://www.intersexuelle -menschen.net/switch-xy.php (Zugriff: 08.07.2014).

Intersexuelle Menschen e. V./Selbsthilfegruppe XY-Frauen.de (2009). Was ist es denn? Intersexualität/DSD. Ratgeber für Hebammen. Hamburg: Eigenverlag. http://www.db.intersexuelle -menschen.net/includes/pdf/Hebammenbroschuere.pdf (Zugriff: 10.08.2014).

Intersexuelle Menschen e.V./XY-Frauen (2008). Schattenbericht zum 6. Staatenbericht der Bunderepublik Deutschland zum Übereinkommen der Vereinten Nationen zur Beseitigung jeder Form der Diskriminierung der Frau (CEDAW). http://www.intersexuelle-menschen. net/parallelberichte/cedaw_2008.php (Zugriff: 07.07.2014).

Intersexuelle Menschen e.V./XY-Frauen (2011). Parallelbericht zum 5. Staatenbericht der Bundesrepublik Deutschland. Übereinkommen gegen Folter und andere grausame, unmenschliche oder erniedrigende Behandlung oder Strafe (CAT). http://www.intersexuelle -menschen.net/pdf/CAT_ParallelReport_Intersex_2011_DE.pdf (Zugriff: 07.07.2014).

Jilg, M. (2007a). Die Katze wäre eher ein Vogel Ein visuelles Hörstück. Dokumentation (55 Min.). Karlsruhe: Staatliche Hochschule für Gestaltung.

Jilg, M. (2007b). Die Katze wäre eher ein Vogel. http://www.die-katze-ist-kein-vogel.de (Zugriff: 13.08.2014).

Jürgen, A. (2013). Über mich. http://www.alexjuergen.at/ (Zugriff: 02.07.2014).

Kampagne Dritte Option (2014a). Der erste »offizielle« Schritt auf dem Weg zu einer dritten Option ist gemacht! http://dritte-option.de/ (Zugriff: 18.08.2014).

Kampagne Dritte Option (2014b): Interview mit einem_r Vertreter_in der Kampagne Dritte Option. Für einen dritten Geschlechtseintrag. Durchgeführt von Manuela Tillmanns am 14.02.2014 in Köln.

Kessler, S. J. (1990). The Medical Construction of Gender: Case Management of Intersexed Infants. Signs 16(1). From Hard Drive to Software: Gender, Computers and Difference, 3-26.

Kleinemeier, E. \& Jürgensen, M. (o.J). Erste Ergebnisse der Klinischen Evaluationsstudie im Netzwerk Störungen der Geschlechtsentwicklung/Intersexualität in Deutschland, Österreich und Schweiz. Januar 2005 bis Dezember 2007. http://www.netzwerk-dsd.uk-sh.de/ fileadmin/documents/netzwerk/evalstudie/Bericht_Klinische_Evaluationsstudie.pdf (Zugriff: 19.08.2014). 
Klöppel, U. (2010). XXOXY ungelöst. Hermaphroditismus, Sex und Gender in der deutschen Medizin. Eine historische Studie zur Intersexualität. Bielefeld: Transcript.

Klöppel, U. (2012a). Intersex im Fokus der Wissenschaft - Anregungen für eine respektvolle Forschung. In I. Lenz, Ilse, K. Sabisch \& M. Wrzesinski (Hrsg.), »Anders und Gleich in NRW«-Gleichstellung und Akzeptanz sexueller und geschlechtlicher Vielfalt. Forschungsstand, Tagungsdokumentation, Praxisprojekte. Studien Netzwerk Frauen- und Geschlechterforschung NRW Nr.15 (S. 92-95). Essen: Eigenverlag.

Klöppel, U. (2012b). Medikalisierung »uneindeutigen« Geschlechts. APuZ. Aus Politik und Zeitgeschichte, 62(20-21). Geschlechtsidentität, 28-33. http://www.bpb.de/apuz/135440/ medikalisierung-uneindeutigen-geschlechts?p=all (Zugriff: 18.08.2018).

Klöppel, U. (2013). Medikalisierung »uneindeutigen« Geschlechts. In E. Barth, B. Böttger, D.C. Ghattas \& I. Schneider, Ina (Hrsg.), Inter. Erfahrungen intergeschlechtlicher Menschen in der Welt der zwei Geschlechter (S. 87-95.). Berlin: NoNo.

Kohler, K. J. (1995). Einführung in die Phonetik des Deutschen. Berlin: Erich Schmidt Verlag.

Kompetenzzentrum geschlechtergerechte Kinder- und Jugendhilfe Sachsen-Anhalt e. V. (2014). Gelbe Reihe. http://www.geschlechtergerechtejugendhilfe.de/publikationen/gelbe -reihe/(Zugriff: 30.06.2014).

Kromminga, I. A. (2013). Herm Kunst. Das Persönliche ist politisch. In E. Barth, B. Böttger, D.C. Ghattas \& I. Schneider (Hrsg.), Inter. Erfahrungen intergeschlechtlicher Menschen in der Welt der zwei Geschlechter (S. 96-108). Berlin: NoNo.

Lang, C. (2006). Intersexualität. Menschen zwischen den Geschlechtern. Frankfurt: Campus.

Lenz, I., Sabisch, K. \& Wrzesinski, M. (2012). Interdisziplinäre Fachtagung »Anders und Gleich in NRW« - Überblick und Ergebnisse. In I. Lenz, K. Sabisch \& M. Wrzesinski (Hrsg.), »Anders und Gleich in NRW" - Gleichstellung und Akzeptanz sexueller und geschlechtlicher Vielfalt. Forschungsstand, Tagungsdokumentation, Praxisprojekte. Studien Netzwerk Frauen- und Geschlechterforschung NRW Nr.15 (S. 59-65). Essen: Eigenverlag.

Mahoney, M. J. (1991). Human Change Processes. New York: Basic Books.

Marzban, G. (2012). Ein Pate für transsexuelle MigrantInnen. http://www.m-media.or.at/ gesellschaft/ein-pate-fur-transsexuelle-migrantinnen/2012/12/12/ (Zugriff: 02.07.2014).

Mein Geschlecht (o. J.). www.meingeschlecht.de (Zugriff: 14.08.2014).

Morgen, C. (2013). Mein intersexuelles Kind. Weiblich männlich fließend. Berlin: Transit.

Nestmann, F. (2002). Ressourcenförderung in der Studien- und Studentenberatung - Das Dresdner Netzwerk Studentenbegleitender Hilfe. In F. Nestmann \& F. Engel (Hrsg.), Die Zukunft der Beratung (S. 297-322). Tübingen: dgtv.

Nestmann, F. (2008). Die Zukunft der Beratung in der Sozialen Arbeit. Beratung Aktuell, 9(2), 72-96.

Netzwerk Trans*-Inter*-Sektionalität (2013). Intersektionale Beratung von/zu Trans* und Inter*. Ein Ratgeber zu Transgeschlechtlichkeit, Intergeschlechtlichkeit und Mehrfachdiskriminierung. Berlin: Eigenverlag.

Neue Gesellschaft für Bildende Kunst e.V. (Hrsg.). (2005). 1-0-1 [one 'o one] intersex. Das ZweiGeschlechter-System als Menschenrechtsverletzung. Berlin: NGBK.

Organisation Intersex International Deutschland (o.J.). http://www.intersexualite.de/index.php/ themen/aktivitaten/bundesweit/ (Zugriff: 07.07.2014).

Organisation Intersex International (2013). Aus aktuellem Anlass:Verpflichtende Offenlassung der Geschlechtszugehörigkeit tritt am 1.11.2013 in Kraft. http://www.intersexualite.de/index. php/aus-aktuellem-anlass-verpflichtende-offenlassung-geschlechtszugehoerigkeit-tritt -am-1-11-2013-in-kraft/ (Zugriff: 07.07.2014). 
ORQOA - Oriental Queer Organisation Austria (2012). Hilfe durch das NETZWERK. http://orqoa. at/netzwerk.html (Zugriff: 02.07.2014).

Perko, G. (2005). Queer-Theorien. Ethische, politische und logische Dimensionen plural-queeren Denkens. Köln: PapyRossa.

Plattner, K. (2008). Erfahrungen der Mutter eines intersexuellen Kindes. In M. Groneberg \& K. Zehnder (Hrsg.), »Intersex Geschlechtsanpassung zum Wohle des Kindes? Erfahrungen und Analysen (S. 13-17). Freiburg: Academic Press.

Praunheim, R.v. (1970). Nicht der Homosexuelle ist pervers, sondern die Situation in der er lebt. Spielfilm (67 Min.). Deutschland.

Prensky, M. (2001). Digital Natives, Digital Immigrants. On the Horizon, 9(5). http://www.nnstoy. org/download/technology/Digital\%20Natives\%20-\%20Digital\%20lmmigrants.pdf (Zugriff: 18.08.2014).

Psychenet (2013). Informationsflyer »Peer-Beratung«. http://www.uke.de/kliniken/psychiatrie/ downloads/klinik-psychiatrie-psychotherapie/Download.Informationsflyer_ PeerBeratung.pdf (Zugriff: 19.08.2014).

Puenzo, L. (2007). XXY. Spielfilm (86 Min.). Argentinien/Frankreich/Spanien.

Pulvermüller, J.M. (2012). Gedanken einer Mutter. In K. Schweizer \& H. Richter-Appelt (Hrsg.), Intersexualität kontrovers. Grundlagen, Erfahrungen, Positionen (S. 255-267). Gießen: Psychosozial-Verlag.

Reiter, M. (2000a). Medizinische Interventionen als Folter. GiGi - Zeitschrift für sexuelle Emanzipation, 9. http://www.gigi-online.de/intervention9.html (Zugriff: 18.08.2014).

Reiter, M. (2000b). Theoretische Differenz und symbolische Nähe. http://www.gigi-online.de/ differenz6.html (Zugriff: 31.01.2014).

Reiter, M. (2000c). Ein normales Leben ermöglichen. http://www.gigi-online.de/intersex8.html (Zugriff: 14.08.2014).

Reuter, J. (2002). Ordnungen des Anderen. Zum Problem des Eigenen in der Soziologie des Fremden. Bielefeld: Transcript.

Reutlinger, U. (2004). Beratung für Opfer sexueller Gewalt. In F. Nestmann, F. Engel \& U. Sickendieck (Hrsg.), Handbuch der Beratung. Band 2. Ansätze, Methoden und Felder (S. 1187-1199). Tübingen: dgvt.

Sabisch, K. (2014). Geschlechtliche Uneindeutigkeit, soziale Ungleichheit? Zum Alltagserleben von intersexuellen Kindern. Psychosozial, 37(1), 55-61.

Sanders, R. (2004). Die Beziehung zwischen Ratsuchendem und Berater. In F. Nestmann, F. Engel \& U. Sickendieck (Hrsg.), Handbuch der Beratung. Band 2. Ansätze, Methoden und Felder (S. 797-807). Tübingen: dgvt.

Scharang, E. (2006a). Tintenfischalarm. Spielfilm (107 Min.).Österreich.

Scharang, E. (2006b). Presseheft zum Film Tintenfischalarm. URL: http://www.film-kultur.de/ glob/tintenfischalarm_ph2.pdf (Zugriff: 18.08.2014).

Schiersmann, C. \& Thiel, H.-U. (2004). Beratung in der Weiterbildung. In F. Nestmann, F. Engel \& U. Sickendieck (Hrsg.), Handbuch der Beratung. Band 2. Ansätze, Methoden und Felder (S. 891-906). Tübingen: dgvt.

Schönbucher, V., Schweizer, K., Brunner, F., Schützmann, K., Rustige, L. \& Richter-Appelt, H. (2010). Sexual quality of life in $46, X Y$ individuals with DSD. Journal of Sex Medicine. Epub online first.

Schützmann, K.; Brinkmann, L.; Schacht, M. \& Richter-Appelt, H. (2009). Psychological distress, self-harming behaviour and suicidal tendencies in adult persons with disorders of sex development. Archives of Sexual Behaviour 38 (1), 16-33. 
Schweizer, K. (2012). Sprache und Begrifflichkeiten. Intersexualität benennen. In K. Schweizer \& H. Richter-Appelt (Hrsg.), Intersexualität kontrovers. Grundlagen, Erfahrungen, Positionen (S. 19-39). Gießen: Psychosozial-Verlag.

Schweizer, K. \& Richter-Appelt, H. (2012a). Behandlungspraxis gestern und heute. Vom »optimalen Geschlecht« zur individuellen Indikation. In K. Schweizer \& Richter-Appelt, H. (Hrsg.), Intersexualität kontrovers. Grundlagen, Erfahrungen, Positionen (S. 99-118). Gießen: Psychosozial-Verlag.

Schweizer, K. \& Richter-Appelt, H. (2012b). Die Hamburger Studie zur Intersexualität. Ein Überblick. In K. Schweizer \& H. Richter-Appelt (Hrsg.), Intersexualität kontrovers. Grundlagen, Erfahrungen, Positionen (S. 187-205). Gießen: Psychosozial-Verlag.

Sebastian (2013). Meine erste Intersex-Geschichte. In E. Barth, B. Böttger, D. C. Ghattas \& I. Schneider (Hrsg.), Inter. Erfahrungen intergeschlechtlicherMenschen in der Welt der zwei Geschlechter (S. 11-14). Berlin: NoNo.

Seelenlos (2012). Intersex-Genitalverstümmelungen (IGMs) in Kinderkliniken: Typische Diagnosen und Eingriffe. http://blog.zwischengeschlecht.info/post/2012/03/23/ Genitalverstuemmelung-typische-Diagnosen-und-Eingriffe (Zugriff: 08.07.2014).

Sexualforschung Hamburg (o. J.). http://www.sexualforschung-hamburg.de/. (Zugriff: 19.08.2014). SHG Eltern (o. J.). URL: http://eltern.shg.intersexuelle-menschen.net (Zugriff 08.07.2014).

SHG XY-Frauen (o. J.). https://xy-frauen.beranet.info/ (Zugriff: 08.07.2014).

Sickendiek, U., Engel, F. \& Nestmann, F. (1999). Beratung. Eine Einführung in sozialpädagogische und psychosoziale Beratungsansätze. Weinheim/München: Juventa.

Sickendiek, U. (2004). Feministische Beratung. In F. Nestmann, F. Engel \& U. Sickendieck (Hrsg.), Handbuch der Beratung. Band 2. Ansätze, Methoden und Felder (S. 765-779). Tübingen: dgvt.

Smykalla, S. (2011). Gender und Diversity im Diskurs von Weiterbildung und Beratung - Ansatzpunkte für Perspektiven der Intersektionalität. In S. Smykalla \& D. Vinz (Hrsg.), Intersektionalität zwischen Gender und Diversity. Theorien, Methoden und Politiken der Chancengleichheit (S. 231-245). Münster: Westfälisches Dampfboot.

Smykalla, S. \& Vinz, D. (Hrsg.). (2011). Intersektionalität zwischen Gender und Diversity. Theorien, Methoden und Politiken der Chancengleichheit. Münster: Westfälisches Dampfboot.

Spacefemfm (2014). Hoch die intersexuelle Solidarität. Radiosendung vom 20.06.2014. http:// cba.fro.at/261723 (Zugriff: 02.07.2014).

Spivak, G.C. (1985). The Rani of Simur: An Essay in Reading the Archives. History and Theory, 24(3), 247-272.

Stark, W. (2004). Beratung und Empowerment - empowerment-orientierte Beratung? In F. Nestmann, F. Engel \& U. Sickendiek (Hrsg.), Das Handbuch der Beratung. Band 1: Disziplinen und Zugänge (S. 535-546). Tübingen: dgvt.

Stecklina, G. \& Böhnisch, L. (2004). Beratung von Männern. In F. Nestmann, F. Engel \& U. Sickendiek (Hrsg.), Das Handbuch der Beratung. Band 1: Disziplinen und Zugänge (S. 219-230). Tübingen: dgvt.

Tatschmurat, C. (2004). Gender Troubles in der Beratung. In F. Nestmann, F. Engel \& U. Sickendiek (Hrsg.), Das Handbuch der Beratung. Band 1: Disziplinen und Zugänge (S. 231-243). Tübingen: dgvt.

Thiersch, H. (2004a). Sozialarbeit/Sozialpädagogik und Beratung. In F. Nestmann, F. Engel \& U. Sickendiek (Hrsg.), Das Handbuch der Beratung. Band 1: Disziplinen und Zugänge (S. 115-124). Tübingen: dgvt.

Thiersch, H. (2004b). Lebensweltorientierte Soziale Beratung. In F. Nestmann, F. Engel \& U. Sicken- 
diek (Hrsg.), Handbuch der Beratung. Band 2. Ansätze, Methoden und Felder (S. 699-709). Tübingen: dgvt.

Time, J. \& Franzen, J. (Hrsg.). (2012). trans*__ Homo. Differenzen, Allianzen, Widersprüche. Differences, Alliances, Contradictions. Berlin: NoNo.

Tolmein, O. \& Rotermund, B. (o.J.): Das verordnete Geschlecht. http://www.das-verordnete -geschlecht.de/ (Zugriff: 13.08.2014).

Tolmein, O., Rotermund, B., Reiter, M. \& Müller E. (2001). Das verordnete Geschlecht. Wie aus Zwittern Männer und Frauen gemacht werden. Dokumentation (62 Min.). Hamburg: Abbildungszentrum.

Tolmein, O. (2012). Intersexualität. Mein Geschlecht gehört mir. http://www.faz.net/ intersexualitaet-mein-geschlecht-gehört-mir-11659997.html (Zugriff: 12.01.2015).

Tolmein, O. (2014). Das Dritte Geschlecht. Weder Frau noch Mann: Intersexuelle im Notstand. In Frankfurter Allgemeine Zeitung (FAZ) vom 06 .August 2014, Nr. 180. S. 12.

Truffer, D. (2011). Zur Situation von Menschen mit Intersexualität in Deutschland. Öffentliche Anhörung vom 08. Juni 2011. Deutscher Ethikrat. http://www.ethikrat.org/dateien/pdf/ anhoerung-08-06-2011-truffer.pdf (Zugriff: 12.08.2014).

Truffer, D. \& Seelenlos (2011). Interview mit Zwischengeschlecht.org. Radiosendung vom 15.11.2011. http://www.freie-radios.net/44292 (Zugriff: 09.07.2014).

Tuggener, H. (1983). Der Klient - Versuch über den Bedeutungswandel eines Begriffs. In S. StaubBerasconi (Hrsg.), Theorie und Praxis der Sozialen Arbeit (S. 52f.). Bern/Stuttgart: Haupt Verlag.

Tuider, E. (2008). Diversität von Begehren, sexuellen Lebensstilen und Lebensformen. In E.B. Schmidt \& U. Sielert (Hrsg.), Handbuch der Sexualpädagogik und sexuelle Bildung (S. 251-260). Weinheim/München: Juventa.

Veith, L. (2012). Der Verein »Intersexuelle Menschen e. V.«. In I. Lenz, K. Sabisch \& M. Wrzesinski (Hrsg.), »Anders und Gleich in NRW - Gleichstellung und Akzeptanz sexueller und geschlechtlicher Vielfalt. Forschungsstand, Tagungsdokumentation, Praxisprojekte. Studien Netzwerk Frauen- und Geschlechterforschung NRW Nr.15 (S. 95-96). Essen: Eigenverlag.

Veith, L. (2014): Interview mit Lucie Veith vom Verein Intersexuelle Menschen. Durchgeführt von Manuela Tillmanns am 17.02.2014 in Neu-Wulmstorf.

VIMÖ - Verein Intersexueller Menschen Österreich (2014). Über uns. http://vimoe.at/ueber-uns/ (Zugriff:30.06.2014).

VIMÖ - Verein Intersexueller Menschen Österreich (o. J.). Statuten des Vereins VIMÖ. http://vimoe. at/wp-content/uploads/2014/03/VIMO\%CC\%88_statuten.pdf (Zugriff: 30.06.2014).

Vogt, I. (2004). Frauen und Beratung. In F. Nestmann, F. Engel \& U. Sickendiek (Hrsg.), Das Handbuch der Beratung. Band 1: Disziplinen und Zugänge (S. 209-218). Tübingen: dgvt.

Völling, C. (2010). Ich war Mann und Frau. Mein Leben als Intersexuelle. Köln: Fackelträger.

Voß, H.-J. (2011). Geschlecht. Wider die Natürlichkeit. Stuttgart: Schmetterling.

Voß, H.-J. (2012a). Intersexualität - Intersex. Eine Intervention. Münster: Unrast.

Voß, H.-J. (2012b). Intersexualität. Varianzen der Geschlechtsentwicklung. http://www.querelles -net.de/index.php/qn/article/view/1030/1057 (Zugriff: 19.08.2014).

Voß, H.-J. (2013). Das Ende des Sex. Intersexualität und Personenstand: ohne Geschlechtseintrag als problematische »Muss-Bestimmung«. http://dasendedessex.de/tag/personenstand/ (Zugriff: 30.03.2014).

Voß, H.-J. (2014). Intergeschlechtlichkeit - Aktivismus und Forschung, ihre Verzahnung und intersektionale Fortentwicklung. In Bundesstiftung Magnus Hirschfeld (Hrsg.), Forschung im Queerformat - Aktuelle Beiträge der LSBTI*-, Queer und Geschlechterforschung (S. 117-131). Bielefeld: Transcript. 
Wiesendanger, K. (2004). Beratung für Lesben und Schwule. In F. Nestmann, F. Engel \& U. Sickendiek (Hrsg.), Das Handbuch der Beratung. Band 1: Disziplinen und Zugänge (S. 245-254). Tübingen: dgvt.

Wunder, M. (2012). Intersexualität: Leben zwischen den Geschlechtern. APuZ. Aus Politik und Zeitgeschichte, 62(20-21). Geschlechtsidentität, 34-40. http://www.bpb.de/apuz/135442/ intersexualitaet-leben-zwischen-den-geschlechtern?p=all (Zugriff: 18.08.2014).

XY-Frauen (o. J.). http://www.xy-frauen.de/ (Zugriff: 12.08.2014).

Zehnder, K. (2008). Intersexualität als soziales Phänomen. Handlungsbedarf aus sozialarbeiterischer Perspektive auf der Grundlage einer Inhaltsanalyse persönlicher Geschichten aus dem World Wide Web. In M. Groneberg \& K. Zehnder (Hrsg.), »Intersex« Geschlechtsanpassung zum Wohle des Kindes? Erfahrungen und Analysen (S. 25-52). Freiburg: Academic Press.

Zehnder, K. (2010). Zwitter beim Namen nennen. Intersexualität zwischen Pathologie, Selbstbestimmung und leiblicher Erfahrung. Bielefeld: Transcript.

Zehnder, K. (2011). »Man hat ich so beschädigt«. Zur unterschiedlichen Deutung von Verletzbarkeit und Verletzung am Beispiel medizinischer Eingriffe in intersexuelle Körper. Feministische Studien, 2011/2, 248-263.

Zwicker-Pelzer, R. (2010). Beratung in der Sozialen Arbeit. Regensburg: Klinkhardt.

Zwischengeschlecht.org (2010a). http://blog.zwischengeschlecht.info/post/2010/10/17/\%22Tabu -Intersexualitaet\%22-online-auf-arte-tv (14.08.2014)).=rNg8NhVwb5s (Zugriff: 19.08.2014).

Zwischengeschlecht.org (2010b). Statuten. URL: http://zwischengeschlecht.org/public/ Statuten_Zwischengeschlecht.pdf (Zugriff: 07.07.2014).

Zwischengeschlecht.org (2010c). Verein. URL: http://zwischengeschlecht.org/category/Verein (Zugriff: 07.07.2014). 


\section{Psychosozial-Verlag}

Katinka Schweizer, Hertha Richter-Appelt (Hg.)

Intersexualität kontrovers

Grundlagen, Erfahrungen, Positionen

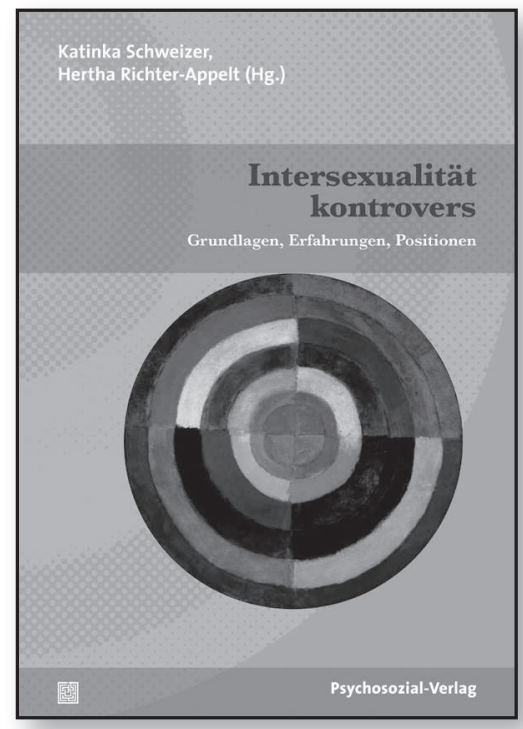

$2012 \cdot 524$ Seiten $\cdot$ Broschur

ISBN 978-3-8379-2188-5
Es gibt weltweit zahlreiche Menschen, die nicht eindeutig einem biologischen Geschlecht zuzuordnen sind - sie sind intersexuell.

Die Auseinandersetzung mit dem Phänomen der Intersexualität wirft Fragen der Grenzüberschreitung und der Zuständigkeit für Entscheidungsprozesse im medizinischen Bereich auf. Nicht nur verschiedene Behandlungsmaßnahmen können Leid bei den Betroffenen erzeugen, sondern auch Geheimhaltung, Diskriminierung und Ausgrenzung.

Der vorliegende Band versammelt aktuelles Grundlagenwissen für ein vertieftes Verständnis des tabuisierten Phänomens und seiner verschiedenen Erscheinungsformen. Internationale Wissenschaftler, Praktiker und Experten geben in trans- und interdisziplinären Beiträgen einen differenzierten Einblick in die kontroversen Positionen im medizinischen, psychosozialen und gesellschaftlichen Umgang mit Intersexualität. Ein informativer Anhang mit wichtigen Adressen, Literatur- und Filmhinweisen ergänzt die verständliche und umfassende fachliche Diskussion. 


\title{
属 Psychosozial-Verlag
}

\section{Jean Lessenich \\ Die transzendierte Frau}

\author{
Eine Autobiografie
}

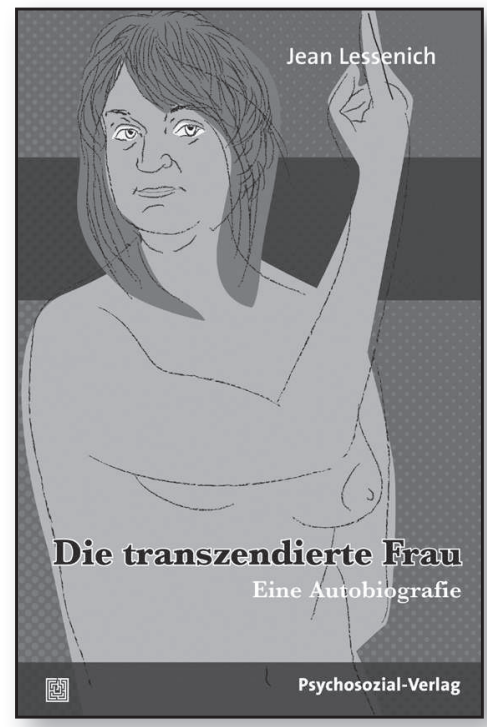

$2012 \cdot 219$ Seiten $\cdot$ Broschur

ISBN 978-3-8379-2170-O
"Ich bin siebenundsechzig und starre in den Spiegel. Von dort starrt die Realität auf mich zurück.

Ich bin das, was man eine Transsexuelle nennt. Ich wurde bei meiner Geburt als männlich einsortiert und lebe nun mein Leben als etwas anderes. Vor vierzig Jahren unterzog ich mich in Casablanca einer Operation, die aus meinem männlichen Körper einen weiblichen machte - und mich von einem heterosexuellen Mann in eine lesbische Frau verwandelte.«

Aus Liebe entschied sich Jean Lessenich zwölf Jahre nach ihrer Geschlechtsumwandlung, wieder als Mann zu leben. Dies schien ihr der einzige Weg zu sein, ihrer japanischen Lebensgefährtin einen dauerhaften Aufenthalt in Deutschland zu ermöglichen. Heute, fünfzehn Jahre nach deren Tod, lebt sie wieder als lesbische Frau.

Jenseits aller Klischees führt uns diese Autobiografie vor Augen, dass das Leben als Transsexuelle kein Hollywood-Film ist. Es verspricht kein Frauenglück nach entsprechender OP, zeigt aber, dass es sich lohnt, den eigenen Weg zu gehen. 


\section{監 Psychosozial-Verlag}

\section{Alexandra Köbele Ein Junge namens Sue Transsexuelle erfinden ihr Leben}

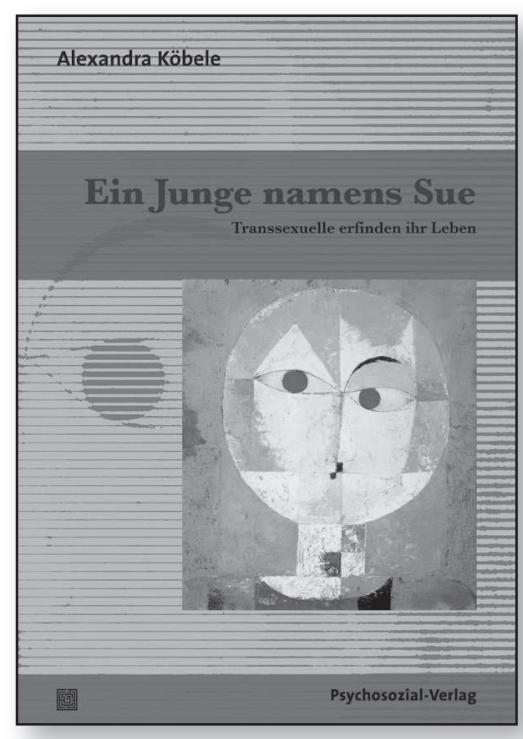

$2011 \cdot 282$ Seiten $\cdot$ Broschur ISBN 978-3-8379-2125-O

»Ein erfrischend anderes Buch über Transsexualität, das eine Menge Fragen zum Weiterdenken aufwirft.» Elke Heinicke, Lesbenring-Info 1/2012
Die Geschichte unseres Lebens erzählen wir unterschiedlich. Wann und wem erzählen wir sie? Welche Fragen werden uns gestellt? Auf welche Themen legen wir den Schwerpunkt? Möglicherweise haben Familie, Schulerfahrungen, Karriere und Freundschaften mit unserer Identität nicht annähernd so viel zu tun wie das Thema der geschlechtlichen Identität - die für die meisten Menschen selbstverständlich ist -, und alle anderen Themen werden auf dieser Basis gesehen.

Im vorliegenden Buch schildern fünf Transsexuelle ihren Lebensweg. Sie erzählen vor dem Hintergrund ihrer Geschlechtsidentität und konstruieren ihre Biografie rund um den Wunsch und die Notwendigkeit, das Körpergeschlecht dem empfundenen, der inneren Identität anzupassen. Fünf Lebensgeschichten zeigen ungewöhnliche Wege der Identitätsfindung. 


\section{鹫, Psychosozial-Verlag}

\section{Marlen Bidwell-Steiner, Anna Babka (Hg.) \\ Obskure Differenzen \\ Psychoanalyse und Gender Studies}

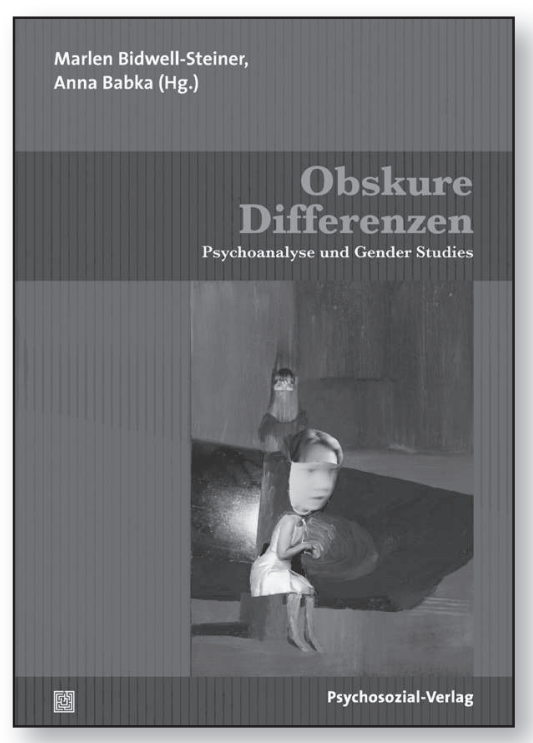

$2013 \cdot 272$ Seiten $\cdot$ Broschur ISBN 978-3-8379-2271-4

Melancholie, Verdrängung, Fetisch, Maskerade, Libido: psychoanalytische Theoreme oder Repertoire der Gender Studies? Eine Erkundung der Gemeinsamkeiten und Unterschiede!

Gegenwärtig werden Geschlechtsidentitäten in vielen Kulturen als fragil und wandelbar erlebt. Um den damit verbundenen individuellen und kollektiven Erfahrungen zwischen Angst und Neugier besser begegnen zu können, ist eine Zusammenführung von Psychoanalyse und Gender Studies naheliegend und notwendig. Ihre genuine Hinwendung zu Fragen der geschlechtlichen Identifizierung und der gemeinsame Anspruch auf ein Ineinandergreifen von Theorie und Praxis implizieren vielfältige Anschlussmöglichkeiten.

Doch trotz innovativer theoretischer Ansätze innerhalb beider Fachrichtungen ist es nicht leicht, diese ineinander zu überführen und produktiv zu machen. Durch ihre Expertise in beiden Forschungsrichtungen gelingt es den BeiträgerInnen, ein produktives Spannungsverhältnis zwischen der psychoanalytischen Praxis und den Ansätzen der Gender Studies herzustellen und die wechselseitige Rezeption zu stärken. Zugleich werden die obskuren Differenzen beleuchtet und verborgene Parallelen herausgearbeitet. 


\section{望 Psychosozial-Verla:}

\section{Anna Sieben, Julia Scholz \\ (Queer-)Feministische Psychologien}

Eine Einführung

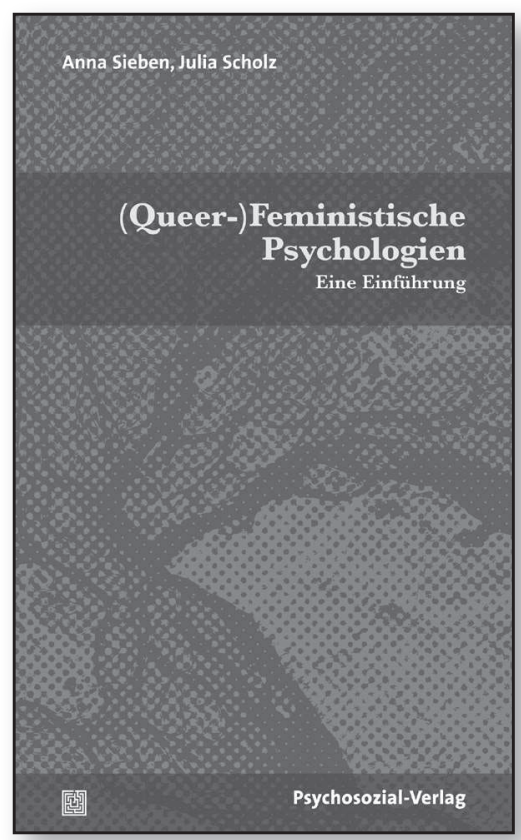

$2012 \cdot 181$ Seiten $\cdot$ Broschur ISBN 978-3-8379-2208-O
Queer-feministische Psychologien sind den politischen Anliegen des Feminismus und des Queer-Aktivismus verpflichtet: der Überwindung von Ungleichheit und Unterdrückung, die an das Geschlecht und/oder die Sexualität einer Person geknüpft sind. In diesem Forschungsgebiet werden einerseits psychologische Theorien genutzt, um Macht, Normativität, Sexualität und Geschlechtlichkeit zu untersuchen. Andererseits werden psychologische Ansätze (selbst-)kritisch auf ihren anti-emanzipatorischen Gehalt hin überprüft.

In diesem Buch werden fünf unterschiedliche, vor allem angloamerikanische Forschungsansätze der queerfeministischen Psychologien vorgestellt, systematisiert und dem deutschsprachigen Lesepublikum zugänglich gemacht. Für alle, die sowohl an Feminismus und queeren Politiken als auch an Psychologie interessiert sind, stellt es eine einmalige Einführung in das Feld dar. 
Menschen, die nicht eindeutig dem männlichen oder weiblichen Geschlecht zugeordnet werden können, sind gesellschaftlichen Stigmatisierungen und medizinisch-psychologischen Pathologisierungen ausgesetzt. Zentrale Studien im deutschsprachigen Raum bestätigen die Notwendigkeit eines fundamentalen Paradigmenwechsels im gesellschaftlichen Umgang mit Intergeschlechtlichkeit bzw. Intersexualität. Zwar hat die Auseinandersetzung mit dem Thema bereits Einzug in den Bildungsbereich erhalten, jedoch existieren bislang keine Veröffentlichungen zur professionellen Beratung von Inters*.

Mithilfe der Analyse von Selbstdarstellungen und Expert_innen-Interviews werden im vorliegenden Buch konkrete
Impulse und Handlungsempfehlungen für eine inter*-spezifische Beratungspraxis erarbeitet. Interdisziplinarität, Ressourcenorientierung und Personenzentriertheit werden als wichtige Grundpfeiler benannt, die es ermöglichen, neue methodische Denk- und Handlungsmuster zu formulieren. Dabei werden auch bisher bestehende Beratungsansätze und -konzepte aufgegriffen und unter Einbezug von Peers und Selbsthilfegruppen erweitert. So ergeben sich neue methodische Zugänge und Perspektiven, die sich an den Bedürfnissen und Wünschen intergeschlechtlicher Personen orientieren und ihnen Formen der Selbstermächtigung sowie barrierefreie Räume für selbstbestimmtes Agieren eröffnen.

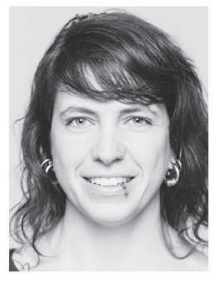

Manuela Tillmanns ist Sonderpädagog_in und Sexualwissenschaftler_in (M.A.) und arbeitet als Lehrbeauftragte_r an der Universität zu Köln. Tillmanns war Sozialarbeiter_in beim Kölner Straßenstrich-Projekt Geestemünder Straße und wissenschaftliche_r Mitarbeiter_in im Lehr-Forschungs-Projekt "Let's talk about Sex" an der Universität zu Köln. Forschungsschwerpunkte: Intergeschlechtlichkeit, sexualisierte Gewalt, sexuelle Bildung, Sexarbeit, Pornografie und hegemoniale Männlichkeiten. 